\title{
Modulation of vascular inflammation: cell type specific effects by ADAMs and HDL
}

Citation for published version (APA):

van der Vorst, E. P. C. (2015). Modulation of vascular inflammation: cell type specific effects by ADAMs and HDL. [Doctoral Thesis, Maastricht University]. Maastricht University. https://doi.org/10.26481/dis.20150521ev

Document status and date:

Published: 01/01/2015

DOI:

10.26481/dis.20150521ev

Document Version:

Publisher's PDF, also known as Version of record

\section{Please check the document version of this publication:}

- A submitted manuscript is the version of the article upon submission and before peer-review. There can be important differences between the submitted version and the official published version of record.

People interested in the research are advised to contact the author for the final version of the publication, or visit the DOI to the publisher's website.

- The final author version and the galley proof are versions of the publication after peer review.

- The final published version features the final layout of the paper including the volume, issue and page numbers.

Link to publication

\footnotetext{
General rights rights.

- You may freely distribute the URL identifying the publication in the public portal. please follow below link for the End User Agreement:

www.umlib.nl/taverne-license

Take down policy

If you believe that this document breaches copyright please contact us at:

repository@maastrichtuniversity.nl

providing details and we will investigate your claim.
}

Copyright and moral rights for the publications made accessible in the public portal are retained by the authors and/or other copyright owners and it is a condition of accessing publications that users recognise and abide by the legal requirements associated with these

- Users may download and print one copy of any publication from the public portal for the purpose of private study or research.

- You may not further distribute the material or use it for any profit-making activity or commercial gain

If the publication is distributed under the terms of Article $25 \mathrm{fa}$ of the Dutch Copyright Act, indicated by the "Taverne" license above, 


\section{Modulation of vascular inflammation}

Cell-type specific effects by ADAMs and HDL

Emiel P.C. van der Vorst 
(C) Emiel P.C. van der Vorst, Maastricht 2015

All rights reserved. No part of this thesis may be reproduced, stored in a retrieval system of any nature, or transmitted in any form or by any means, electronic, mechanical, photocopying, recording or otherwise withour prior written permission of the author.

ISBN: $\quad$ 978-94-6295-165-5

Cover design: Emiel P.C. van der Vorst

Layout: $\quad$ Emiel P.C. van der Vorst

Printed by: Proefschriftmaken.nl - Uitgeverij BOXPress

Published by: Uitgeverij BOXPress, 's-Hertogenbosch 


\title{
Modulation of vascular inflammation
}

\section{Cell-type specific effects by ADAMs and HDL}

\author{
PROEFSCHRIFT
}

ter verkrijging van de graad van doctor aan de Universiteit Maastricht, op gezag van de Rector Magnificus, Prof. dr. L.L.G. Soete,

volgens het besluit van het College van Decanen,

in het openbaar te verdedigen op

donderdag 21 mei 2015 om 16.00 uur

door

Emiel Petrus Carla van der Vorst

geboren op 14 augustus 1987 te Sittard. 


\section{Promotores}

Prof. dr. Erik A.L. Biessen

Prof. dr. Menno P.J. de Winther, AMC-UvA, Amsterdam

Prof. dr. Jan F.C. Glatz

\section{Co-promotor}

Dr. Marjo M.P.C. Donners

\section{Beoordelingscommissie}

Prof. dr. Chris P. Reutelingsperger (Voorzitter)

Prof. dr. Philip J. Barter, University of New South Wales, Sydney, Australia Prof. dr. Mat J. Daemen, AMC-UvA, Amsterdam

Prof. dr. Casper G. Schalkwijk

Prof. dr. Christian Weber

Financial support by the Dutch Heart Foundation for the publication of this thesis is gratefully acknowledged. 


\section{Contents}

List of abbreviations

$\begin{array}{lll}\text { Chapter } 1 \text { General Introduction } & 9\end{array}$

Chapter 2 A Disintegrin And Metalloproteases: Molecular scissors in angiogenesis, inflammation and atherosclerosis

Chapter 3 Hematopoietic ADAM8 deficiency does not influence atherosclerotic plaque area or composition, despite its upregulation during plaque progression

Chapter 4 Myeloid ADAM10 deficiency modulates atherosclerotic plaque composition by shifting the balance from inflammation toward fibrosis

Chapter 5 Endothelial ADAM10 deficiency modulates leukocyte recruitment and remarkably augments atherosclerotic lesion development

Chapter 6 High Density Lipoproteins suppress chemokine expression and proliferation in human vascular smooth muscle cells

Chapter 7 High Density Lipoproteins exert pro-inflammatory effects on macrophages via passive cholesterol depletion and PKC-dependent NF-KB/STAT1 activation

Chapter 8 General Discussion

Summary

Samenvatting

Valorisation

Acknowledgements / Dankwoord 


\section{List of abbreviations}

\begin{tabular}{lll} 
AAV & Adeno-accociated virus \\
ABC & ATP binding cassette transporter \\
ACAT & Acyl-CoA: cholesterol ester transferase \\
ADAM & A disintegrin and metalloprotease \\
Apo & Apolipoprotein \\
AU & Arbitrary unit \\
BCA & Bicinchoninic acid \\
BMDM & Bone marrow-derived macrophage \\
BSA & Bovine serum albumin \\
CD & Cluster of differentiation \\
CETP & Cholesteryl ester transfer protein \\
cHDL & Commercial HDL \\
CKII & Casein kinase II \\
CT-B & Cholera toxin subunit B \\
CVD & Cardiovascular disease \\
DHCR24 & 3 3 -Hydroxysteroid- $\Delta$ 24 reductase \\
DMEM & Dulbecco's modified eagles medium \\
EC & Endothelial cell \\
EdU & 5-ethynyl-2'-deoxyuridine \\
EGF & Epidermal growth factor \\
ELISA & Enzyme-linked immunosorbent assay \\
eNOS & Endothelial nitric oxide synthase \\
FBS & Fetal bovine serum \\
FCS & Fetal calf serum \\
FPLC & Fast protein liquid chromatography \\
HCAEC & Human coronary artery endothelial cell \\
HDL & High density lipoproteins \\
HMG CoA & 3-hydroxy-3-methylglutaryl coenzyme A \\
HUVEC & Human umbilical vein endothelial cell \\
HUVSMC & Human umbilical vein smooth muscle cell \\
ICAM1 & Intercellular adhesion molecule 1 \\
IFN & Interferon \\
IL & Interleukin \\
IL-6R & IL-6 receptor \\
IMDM & Iscove modified Dulbecco medium \\
JAM & Junctional adhesion molecule \\
\hline 6 & \\
\hline & \\
\hline
\end{tabular}




\begin{tabular}{ll} 
LCAT & Lecithin:cholesterol transferase \\
LCM & L929-conditioned medium \\
LC-MS/MS & Liquid chromatography-tandem mass spectrometry \\
LDL & Low density lipoproteins \\
LDLr & LDL receptor \\
LPS & Lipopolysaccharide \\
LysM & Lysozyme M \\
M-CSF & Macrophage colony-stimulating factor \\
MI & Myocardial infarction \\
MMP & Matrix metalloproteinase \\
MPLSM & Multiphoton laser scanning microscopy \\
NF-KB & Nuclear factor-kB \\
NIK & NF-kB inducing kinase \\
nHDL & Native HDL \\
OCT & optimum cutting temperature \\
OXLDL & Oxidized-low density lipoproteins \\
p & Phosphorylated \\
PBS & Phosphate buffered saline \\
PCI & Percutaneous coronary interventions \\
PCSK9 & Proprotein convertase subtilisin/kexin type 9 \\
PDGF & Platelet-derived growth factor \\
PECAM1 & Platelet endothelial cell adhesion molecule 1 \\
PKC & Protein kinase C \\
PLOOH & Phospholipid hydroperoxide \\
PLPC & 1-palmitoyl-2-linoleoyl-phosphatidylcholine \\
PLTP & Phospholipid transfer protein \\
PMSF & Phenylmethylsulphonyl fluoride \\
PON-1 & Paraoxonase-1 \\
PRR & Pattern recognition receptor \\
PS & Phosphatidylserine \\
RA & Rheumatoid arthritis \\
RAGE & Receptor for advanced glycation end products \\
RCT & Reverse cholesterol transport \\
rHDL & Reconstituted HDL \\
RIP & Regulated intramembrane proteolysis \\
Scr & Scrambled \\
SEM & Standard error of the mean \\
SMA & Smooth muscle actin \\
SMC & Smooth muscle cell \\
\hline &
\end{tabular}




$\begin{array}{ll}\text { SR } & \text { Scavenger receptor } \\ \text { STAT } & \text { Signal transducer and activator of transcription } \\ \text { TG } & \text { Triglycerides } \\ \text { TIMP } & \text { Tissue inhibitor of metalloproteases } \\ \text { TLR } & \text { Toll-like receptor } \\ \text { TNF- } \alpha & \text { Tumor necrosis factor- } \alpha \\ \text { VCAM1 } & \text { Vascular cell adhesion molecule 1 } \\ \text { VE-cadherin } & \text { Vascular endothelial cadherin } \\ \text { VEGF } & \text { Vascular endothelial growth factor } \\ \text { VLDL } & \text { Very low density lipoproteins } \\ \text { WT } & \text { Wildtype } \\ \text { WTD } & \text { Western-type diet }\end{array}$




\section{Chapter 1}

\section{General Introduction}




\section{Cardiovascular diseases}

The leading cause of death worldwide is cardiovascular disease (CVDs), accounting for 16.7 million deaths annually ${ }^{1,2}$. The most common and most severe CVDs are cerebrovascular disease (e.g. stroke) and coronary artery disease (i.e. myocardial infarction). Both diseases have a major impact on the personal health, but also on the society as a whole. The main underlying cause of CVDs is atherosclerosis, which is a chronic inflammatory disease mainly affecting medium and large-sized arteries ${ }^{3}$. During this pathological process lipids, immune cells and cell debris accumulate in the vessel wall, thereby forming lesions. These initial lesions can progress and grow in size, thereby partially or even fully occluding the vessel, resulting in obstructed blood flow. More often, however, this occlusion and obstructed blood flow is caused by thrombus formation due to the rupture of atherosclerotic lesions. This obstruction can lead to ischemic areas in downstream tissues, most commonly in the heart and in the brain resulting in a myocardial infarction (MI) or stroke, respectively ${ }^{3}$. Many risk factors have already been described for CVDs, including hypertension, smoking, obesity, stress and lack of physical activity ${ }^{1}$. However, to date atherosclerosis is still not curable or reversible with any available drug, warranting additional research in this field. Inflammation is one of the most crucial drivers of atherosclerosis, which can be regulated by the modulation of profiles of lipids and protein posttranslational modifications. This thesis will focus on the modulation of these regulatory molecules. 


\section{Atherosclerosis: a lipid driven, chronic inflammatory disease}

Atherosclerosis is a complex multifactorial pathology affecting mainly the medium and large sized arteries. Formation of atherosclerotic lesions occurs predominantly at predisposed sites, i.e. sites of disturbed laminar flow, like bifurcations and curvatures ${ }^{4}$, thereby disturbing the normal, quiescent state of the endothelium. The resulting increased permeability of the endothelial layer leads to the accumulation of lipids, more specifically low density lipoproteins (LDL), in the subendothelial layer of the arterial wall (Fig. 1) ${ }^{5}$.

LDL particles contain a core of cholesterolylesters and triglycerides surrounded by phospholipids, free cholesterol and apolipoprotein-B100 (Apo-B100). Apo-B100 is synthesized in the liver, assembled into and secreted as very low density lipoproteins (VLDL), which are converted to LDL in the circulation 5. Apo-B100 has a high affinity for proteoglycans and extracellular matrix, resulting in the retention of $L D L$ in the intima ${ }^{6}$. LDL is very susceptible to oxidation, resulting in oxidized-LDL particles (oxLDL). These modified lipids will activate the endothelial cells (ECs) and tissue resident macrophages 7. Monocytes will be attracted to the activated area and transmigrate into the vessel wall, where they differentiate into macrophages ${ }^{8}$. Subendothelial/ extravasated LDL will be oxidized and taken up by resident macrophages via scavenger receptors (SRs), mainly SR-A and cluster of differentiation (CD) $36^{9,10}$. The level of oxidation is important in this process, since highly oxidized LDL display a much higher affinity for these receptors than minimally oxidized LDL ${ }^{11}$. Intracellularly, oxLDL will be hydrolysed into free cholesterol and fatty acids in late endosomes ${ }^{12}$. Free cholesterol is subsequently transported to the endoplasmic reticulum where it undergoes re-esterification to cholesteryl esters by the acyl-CoA:cholesterol ester transferase (ACAT) enzyme ${ }^{13}$. This accumulation of cholesteryl esters will transform the macrophages into foam cells, a characteristic hallmark of early atherosclerosis. Not only the uptake and storage of cholesterol is disturbed, but also the excretion mechanisms. ATP binding cassette transporters A1 and G1 (ABCA1 and $A B C G 1)$, are the major contributors to this cholesterol efflux through reverse cholesterol transport (RCT) ${ }^{14,15}$. Cholesterol will efflux toward high density lipoproteins (HDL), making HDL beneficial for atherosclerosis development 14. HDL's metabolism and additional functions will be further introduced later. Normally, this efflux mechanism is upregulated upon lipid loading, however, 
during hypercholesterolemia this route is compromised, further favouring the conversion of macrophages to foam cells ${ }^{16}$.

This macrophage activation, due to the lipid loading, together with the activation of endothelial cells will also lead to more vascular inflammation, by the secretion of various cytokines, chemokines and adhesion molecules ${ }^{17}$. Inflammation can be further modulated by A Disintegrin And Metalloproteases (ADAMs), which is a group of proteins that are able to shed transmembrane molecules like cytokines and chemokines and thereby modulate inflammation 18. The inflammation will result in the attraction of additional monocytes and other immune cells, like T- and B-lymphocytes and neutrophils to the site of injury and lesion formation ${ }^{8}$.

\section{Monocytes/macrophages: the predominant inflammatory cells in atherosclerosis}

In mice, 2 main subtypes of circulating monocytes exist, i.e. the Ly6 $\mathrm{C}^{\text {hi }} \mathrm{CCR} 2^{\text {hi }}$ (inflammatory) and Ly6Clow $\mathrm{CX}_{3} \mathrm{CR} 1^{\text {hi }}$ (resident or patrolling) monocytes ${ }^{19}$. Ly6 $\mathrm{C}^{\text {hi }}$ inflammatory monocytes form the main subtype that will migrate to the site of injury ${ }^{20}$. The mechanism of monocyte recruitment consists of complex interactions between various adhesion molecules and chemokines. The first step in this cascade is the capture and rolling phase, where various chemokines and selectins on the luminal side of the activated endothelium play a crucial role ${ }^{17,21}$. The second step is the firm adhesion of these monocytes to the endothelium. In this phase, vascular cell adhesion molecule 1 (VCAM1) and intercellular adhesion molecule 1 (ICAM1) present on the endothelium are essential ${ }^{21}$. After firm adhesion, the monocytes will have to transmigrate across the endothelial layer, mainly directed by various chemokines and their receptors ${ }^{22}$. Although many chemokines are implicated in this process, the

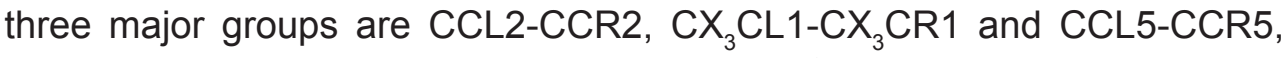
although various receptors have been described for these chemokines ${ }^{23}$. This was further supported by the fact that combined deletion of all three axes in $\mathrm{ApoE}^{-/-}$mice resulted in significant decreases in atherosclerosis development ${ }^{24}$. Next to these chemokines, also the endothelial junction molecules platelet endothelial cell adhesion molecule 1 (PECAM1), VE-Cadherin and junctional adhesion molecules (JAMs) play a crucial role as regulators of endothelial cell permeability and leukocyte transmigration ${ }^{25}$. Once inside the vessel wall, monocytes can differentiate into macrophages, driven by macrophage colonystimulating factor (M-CSF) ${ }^{26}$. Similar to the monocytes, also macrophages are a heterogeneous cell population, consisting of 2 main groups, the 
classically activated, inflammatory M1 macrophages and the alternatively activated, inflammation resolving M2 macrophages ${ }^{27}$. Various cytokines play a role in this polarization of macrophages ${ }^{28}$. Both types of macrophages are present in atherosclerotic lesions, where the balance between them is of great importance for either plaque development or resolving inflammation ${ }^{29}$. These attracted leukocytes will again be exposed to the oxidized-lipid rich environment of the developing lesion, thereby forming foam cells. Thereby a vicious circle of leukocyte attraction and lipid accumulation is formed, stimulating atherosclerosis development.

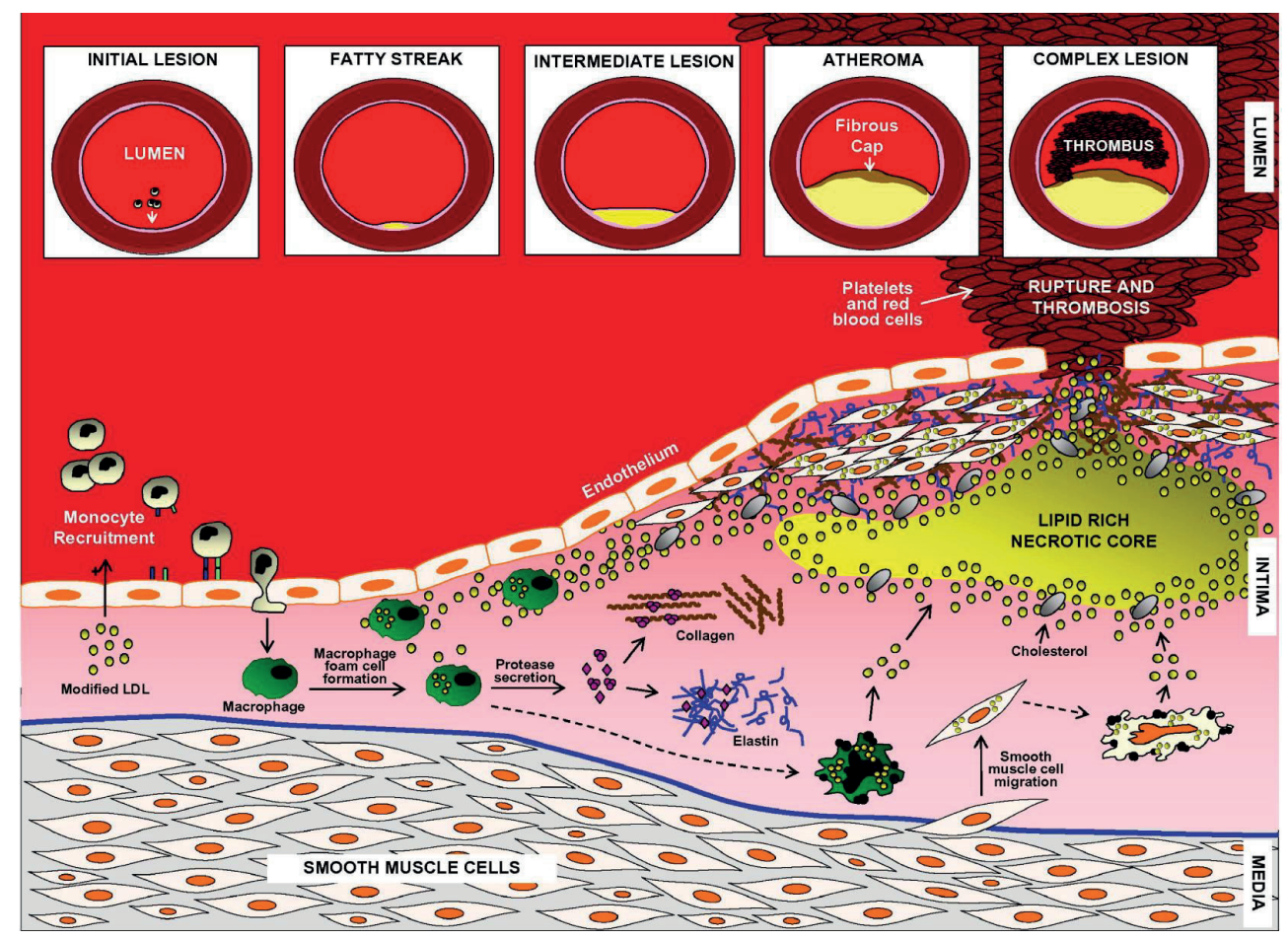

Figure 1. The development of an atherosclerotic lesion ${ }^{17}$. At the site of initial vessel damage, a fatty streak is formed by the accumulation of foamy macrophages. Lesions will further develop by accumulation of lipids and macrophages. Apoptosis of these macrophages will eventually lead to the formation of a necrotic core, resulting in intermediate lesions. Migrated smooth muscle cells into the intima will produce extracellular matrix, forming the fibrous cap. Persisting inflammation, cell recruitment and cell death will further enlarge the necrotic core. Ultimately, deterioration of the fibrous cap by proteases secreted from foamy macrophages will decrease plaque stability and can result in plaque rupture, releasing thrombotic materials into the lumen forming a thrombus and thereby blocking the blood flow. 
When lesional macrophages take up so many lipids and debris, many will eventually go into apoptosis (Fig. 1). In early plaque development the apoptosis will not be that harmful, since neighbouring macrophages will take up and eliminate the apoptotic debris, a process called efferocytosis 29. However, when plaque development progresses, the excessive uptake of lipids and debris continues and eventually leads to cellular stress and impaired efferocytosis ${ }^{30}$. This will result in the accumulation of apoptotic debris and apoptotic macrophages will go into secondary necrosis, leading to the formation of the necrotic core which is characteristic of more advanced lesions ${ }^{29,31}$. The necrotic core will significantly contribute to the lesional inflammation, and thus progression, and also contains pro-thrombotic factors that will lead to a thrombus when it comes into contact with platelets ${ }^{32}$. To prevent this from happening, a fibrous cap is formed between the necrotic core and the lumen, by deposition of mainly collagen and elastin by intimal smooth muscle cells (SMCs) ${ }^{33}$. Various cytokines and growth factors, produced by macrophages and T-lymphocytes, are important for the migration of intimal SMCs to the intima and for the extracellular matrix production ${ }^{33}$. Plaques with a big fibrous cap are considered to be more stable atherosclerotic lesions, i.e. less prone to rupture. However, macrophages can also produce matrix metalloproteinases (MMPs), that are capable of degrading extracellular matrix proteins ${ }^{32}$. Furthermore, it has been shown that macrophages can induce SMC apoptosis, resulting in a decreased matrix production ${ }^{34}$. Fibrous cap degradation and thus thinning makes the lesion more vulnerable and can eventually lead to a plaque rupture, releasing pro-thrombotic material into the bloodstream resulting in thrombus formation and obstruction of blood flow. This can cause ischemia to distal regions and result in a MI or stroke ${ }^{31}$. 


\section{ADAMs in atherosclerosis}

ADAMs are a group of proteins that have various effects on inflammatory mediators, some of which are crucial in the pathogenesis of atherosclerosis. ADAMs consist of many family members and the proteolytically active ADAMs are able to shed / cleave off the extracellular part of transmembrane proteins, thereby releasing an extracellular part which can among others be used to secrete cytokines and chemokines or to inactivate adhesion molecules. This way ADAMs can play a crucial role in leukocyte recruitment, since the chemokines $\mathrm{CX}_{3} \mathrm{CL} 1$ and $\mathrm{CXCL} 16$, the adhesion molecules VCAM1 and ICAM1, but also the junctional molecules JAM-A and VE-Cadherin are all well-known substrates of ADAMs ${ }^{35-39}$. Besides this clear role of ADAMs in inflammation and leukocyte recruitment, ADAMs have also been implicated in angiogenesis (new blood vessel formation), which has been associated with atherosclerotic lesion development and plaque destabilization ${ }^{40}$. Recently, we found that high in vivo expression of ADAM10, a main member of the ADAMs family, is associated with plaque angiogenesis in human atherosclerosis ${ }^{35}$. Although this makes an important role for ADAMs in atherosclerosis development and stability very plausible, a causal role had not been described so far. In chapter 2, the role of ADAMs in angiogenesis and inflammation in relation to atherosclerosis will be reviewed more extensively. 


\section{High Density Lipoproteins and cardiovascular disease}

As introduced above, foam cells play a crucial role in the development of atherosclerosis and the resulting cardiovascular events. Prevention or reversal of this transition from macrophage to foam cell might therefore be used as a therapy for lesion formation ${ }^{41}$. HDL play a crucial role in the RCT, mediating the transport of cholesterol from peripheral tissues, like lesional macrophages, towards the liver for excretion into the faeces ${ }^{42}$. Extensive evidence suggests that HDL indeed play an important role in protection against atherosclerosis, since various clinical and epidemiological studies showed that HDL levels inversely correlate with cardiovascular disease risk ${ }^{43}$. HDL are a very heterogeneous class of lipoproteins, structurally and functionally ${ }^{44}$. In atherosclerosis, functional changes of HDL have been observed, further indicating a role of HDL in this pathogenesis ${ }^{44}$.

\section{HDL heterogeneity and metabolism}

The great heterogeneity of $\mathrm{HDL}$ is mainly attributable to the different content of apolipoproteins and lipids, suggesting also distinct functions ${ }^{45}$. The main constituent of HDL is ApoA-I, accounting for $\sim 70 \%$ of the total HDL protein. The amphipathic, very dynamic structure of ApoA-I, ensuring conformational changes upon lipid binding is also a main contributor to the HDL heterogeneity ${ }^{46}$. ApoA-I is produced and secreted by the liver and the intestines (Fig. 2). The lipid free protein will rapidly interact with cells and obtain phospholipids and free cholesterol, via ABCA1 to form the small, lipidpoor discoidal pre- $\beta$-HDL containing two or three ApoA-I proteins ${ }^{45}$. Another way by which this pre- $\beta-H D L$ is formed, is by lipolysis of VLDL by lipoprotein lipase. Subsequently, lecithin:cholesterol transferase (LCAT) will esterify the cholesterol present in the pre- $\beta-H D L$, forming cholesteryl esters. Since these cholesteryl esters are highly hydrophobic, they will accumulate in the centre of the lipoprotein, forming a lipid core. This process will transform discoidal pre- $\beta$-HDL into spherical $\alpha-H D L{ }^{47}$. Small spherical HDL can further increase in size by taking up free cholesterol from peripheral cells by cholesterol efflux mediated by ABCA1, ABCG1 or SR-B1 and subsequent esterification by LCAT. Additionally, phospholipid transfer protein (PLTP) can transfer phospholipids from VLDL and chylomicrons into HDL, thereby contributing to the remodeling ${ }^{48}$. This phospholipid transfer can also lead to apolipoprotein destabilization, resulting in remnant particle fusion and formation of a larger $\alpha-H D L$ particle ${ }^{49}$. The latter can undergo constituent-exchange with $A p o B$ 
containing lipoproteins (VLDL and LDL) via cholesteryl ester transfer protein (CETP). The net effect of CETP is the transfer of cholesteryl esters from HDL to ApoB containing lipoproteins in exchange for triglycerides (TG), generating TG-rich HDL ${ }^{44,45}$. This CETP mediated transfer will result in the transition of large $\alpha-H D L 2$ particles into smaller $\alpha-H D L 3$ particles. This transition can also be caused either by cholesteryl ester uptake by the liver, mediated by SR-B1 or by hepatic lipase or endothelial lipase mediated hydrolysis of triglycerides 50. The combined action of CETP and lipases results in reduction of HDL size, accompanied by partial loss of ApoA-I from the particle surface. This released lipid-free ApoA-I protein is recycled and will again interact with $A B C A 1$ to start a new lipidation cycle, or will be excreted via the kidneys.

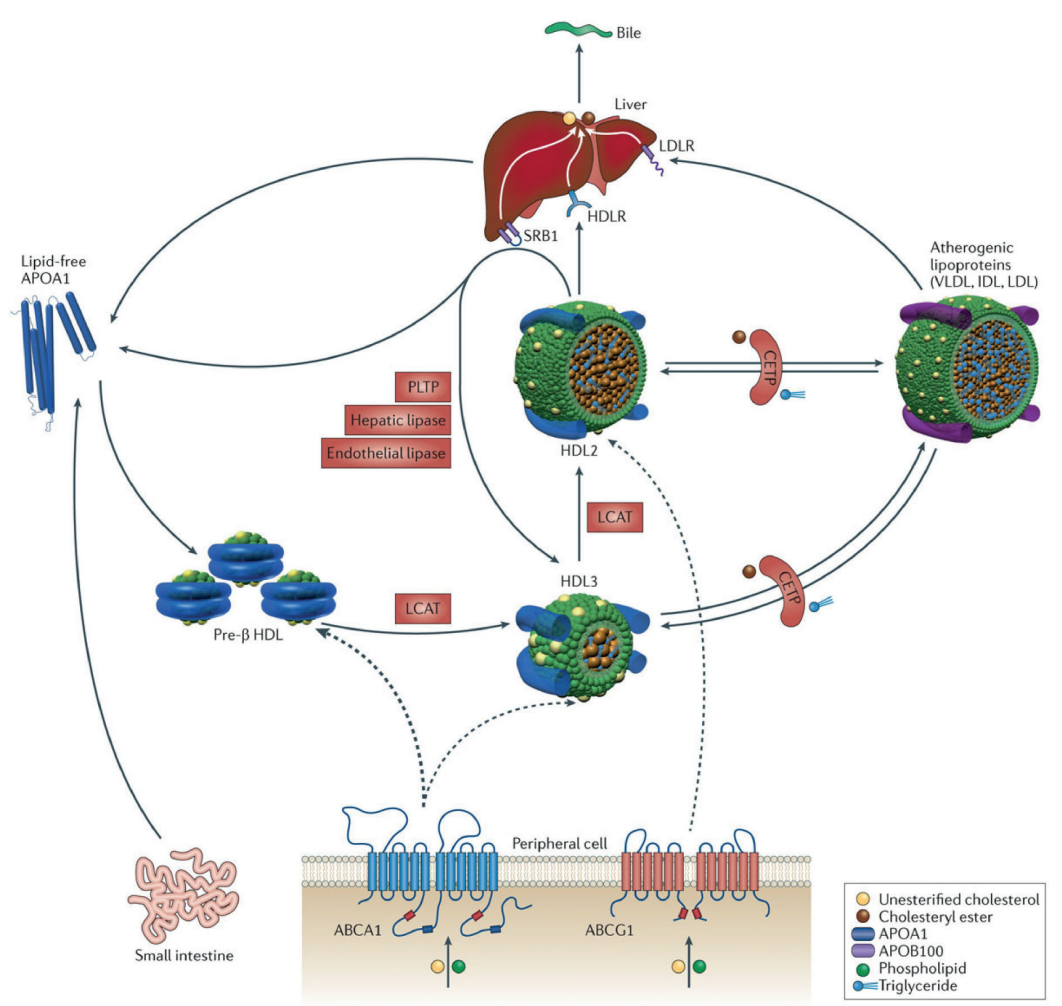

Figure 2. HDL metabolism and reverse cholesterol transport ${ }^{55}$. 
Besides size and lipid composition, HDL subfractions are also distinguished based on their apolipoprotein composition. The two main groups are ApoA-I HDL, which exclusively contain apolipoprotein A-I, and A-I/A-II HDL composed of both ApoA-I and ApoA-II ${ }^{51}$. Even a minor population of ApoE containing HDL has been described ${ }^{52}$. Additionally, HDL can be composed of various other apolipoproteins like ApoC or ApoM ${ }^{53,54}$.

\section{The HDL hypothesis}

Already in 1964, The Framingham Heart Study was the first study showing compelling evidence of an inverse relationship between HDL and cardiovascular disease ${ }^{56}$. This was the start of the concept that HDL is the good cholesterol and could possibly protect against atherosclerosis and cardiovascular disease, in contrast to LDL or the bad cholesterol. This hypothesis was further supported by animal studies during the 1990s, where for example Badimon et al. infused HDL plasma fractions into cholesterol-fed rabbits and observed a regression of atherosclerotic lesions 57. Furthermore, Rubin et al. showed that early atherogenesis was inhibited in mice overexpressing human ApoA-I, resulting in high circulating HDL levels ${ }^{58}$. These studies were in line with the obtained epidemiological data and strengthened the HDL hypothesis, stating that "a reduction of plasma HDL concentration may accelerate the development of atherosclerosis, and hence ischemic heart disease, by impairing the clearance of cholesterol from the arterial wall" 59 .

\section{HDL functions}

\section{Cholesterol efflux capacity}

Over the last years, a lot of studies have focussed on identifying the precise function of HDL that has this protective effect against cardiovascular disease 60. The most studied and best described function of HDL is its role in the RCT. The efflux of cholesterol from peripheral cells, like macrophages, to HDL can be mediated by various pathways. ABCA1 is responsible for the transport of phospholipids and cholesterol from cells towards lipid free ApoA-I or small lipid poor, discoidal HDL ${ }^{61,62}$. Discoidal HDL, but also the larger spherical HDL can act as cholesterol acceptors for ABCG1 mediated efflux ${ }^{61,63}$. These lipid rich HDL particles can also bind and interact with SR-B1. This interaction is bidirectional and the rate of this efflux correlates positively with the HDL cholesterol concentration ${ }^{64}$. The final pathway is receptor-independent passive diffusion of cholesterol along the concentration gradient. This gradient is formed when LCAT esterifies cholesterol that mobilizes towards the centre 
of the HDL particle, thereby depleting the HDL membrane of cholesterol enabling passive diffusion of cholesterol from cells ${ }^{65}$. Andorni et al. even found that this passive pathway had a major contribution to cholesterol efflux in vitro, mostly in cells with normal cholesterol levels ${ }^{65}$. However, the precise contribution of these pathways in vivo remain poorly defined.

\section{Antioxidant activity}

Besides the effects of HDL on cholesterol efflux from cells, HDL particles also have antioxidant activity. Hereby HDL can protect from oxidative damage and modification of LDL ${ }^{66}$. The precise mechanism by which HDL exert this protection remains largely undetermined. A main enzyme of interest in this aspect is paraoxonase-1 (PON-1), which is located on the HDL particle ${ }^{67}$. However, also PON-1 independent antioxidant activity of HDL is observed. Initially phospholipid hydroperoxides (PLOOHs) are transferred from LDL to HDL via CETP ${ }^{68}$. These PLOOHs are subsequently reduced by methionine residues of ApoA-I, forming redox-inactive phospholipid hydroxides ${ }^{69}$.

\section{Anti-inflammatory activity}

HDL have a wide range of anti-inflammatory properties. They can limit the extent of endothelial cell activation, resulting in a decreased adhesion molecule expression such as VCAM-1 and ICAM-1 ${ }^{70}$. Additionally, HDL can suppress chemokine secretion of CCL2, CCL5 and CX ${ }_{3} \mathrm{CL} 1$ from endothelial cells and monocytes, that play a crucial role in monocyte recruitment and atherogenesis ${ }^{71}$. It was even shown that $\mathrm{CCR} 2$ and $\mathrm{CX}_{3} \mathrm{CR} 1$ expression in plaques ofApoA-I injected mice was significantly decreased, further supporting the anti-inflammatory properties of $\mathrm{HDL}{ }^{71}$. This in vivo anti-inflammatory effect of HDL was further observed in normo- and hypercholesterolemic rabbit models showing reduced vascular inflammation and adhesion molecule and chemokine expression ${ }^{72,73}$.

\section{Other activities}

Besides, these three main and well defined functions, HDL have more functions that could contribute to their protective capacity. For example, HDL can inhibit thrombosis by directly or indirectly reducing platelet activation, as well as factors involved in the coagulation cascade, like thrombin and tissue factor ${ }^{74,75}$. HDL can also exert beneficial effects on the endothelium, either by restoring the endothelial function by stimulating endothelial nitric oxide synthase (eNOS) dependent NO production ${ }^{76}$, or by enhancing the recruitment of endothelial progenitor cells to damaged areas to ensure 
proper endothelial repair ${ }^{77}$. Finally, Yvan-Charvet et al. recently showed that HDL supress the proliferation of hematopoietic stem cells, thereby reducing leukocytosis and monocytosis and eventually reducing the diet-induced atherosclerosis ${ }^{78}$.

\section{HDL therapy}

Since HDL have so much beneficial functions and epidemiological data show an inverse relationship with cardiovascular diseases, many efforts are made to increase the plasma HDL concentration in CVD patients. Life style interventions, like increasing the physical activity have been shown to increase HDL concentrations ${ }^{79}$. Besides these interventions, also various therapeutic modalities have been investigated. The main therapeutic strategies that were and are still being investigated will be introduced here (Fig. 3).

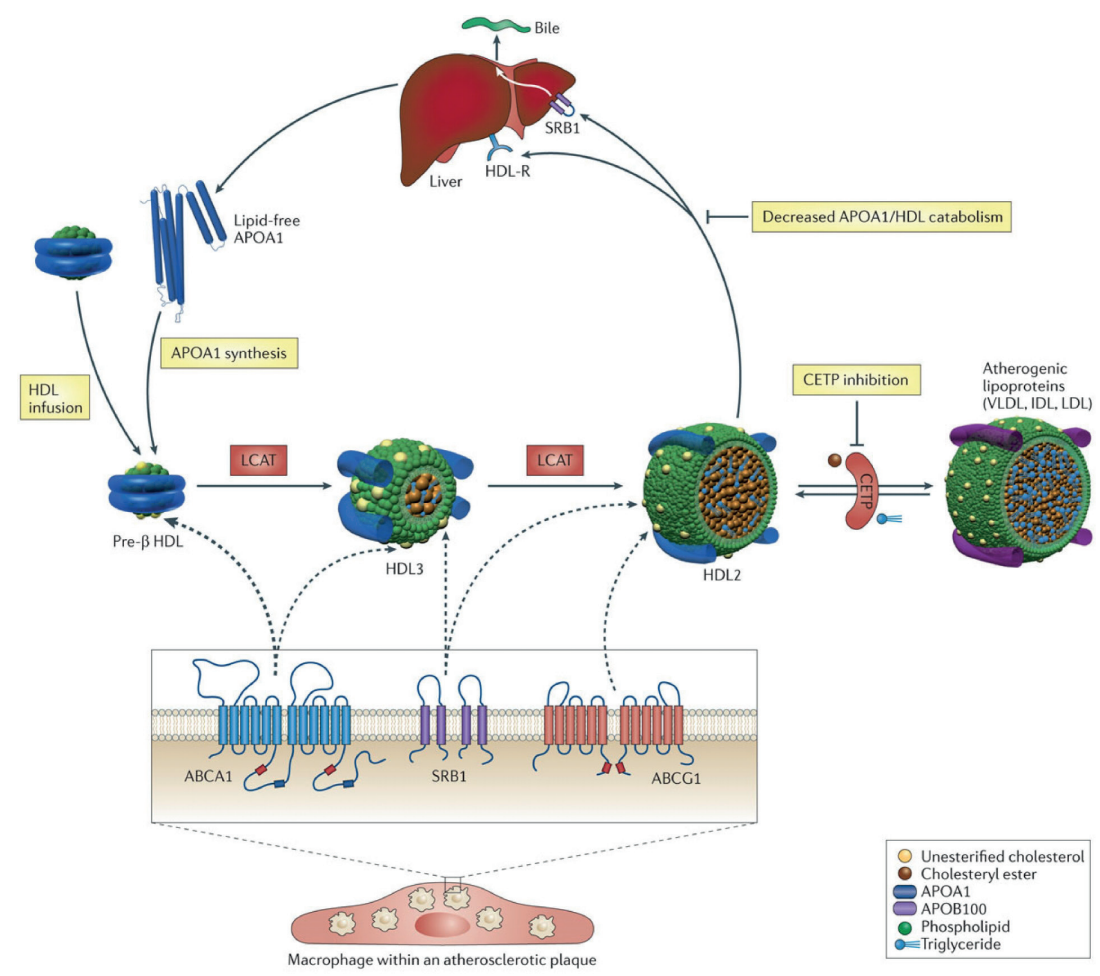

Figure 3. Mode of action of currently available HDL-raising therapies ${ }^{55}$. 
Niacin, or nicotinic acid, is the most effective agent to raise HDL levels and has already been used for more than fifty years to modulate lipids ${ }^{80}$, but it also significantly decreases LDL and triglyceride levels. The mechanism of action of niacin is not very well understood. Some studies show that niacin can reduce direct ApoA-I catabolism ${ }^{81}$, but also inhibit CETP ${ }^{82}$. In recent trials, this beneficial effect of niacin on HDL levels was indeed observed, although without reduced cardiovascular risk ${ }^{83-86}$. Also the latest trial, the Second Heart Protection Study - Treatment of HDL to Reduce the Incidence of Vascular Events (HPS2-THRIVE) trial, where statins and niacin treatment was combined with laropiprant, a prostanoid receptor antagonist, to prevent the niacin mediated vasodilatation causing the occurrence of flushing and itching ${ }^{87}$, showed beneficial lipid modifications without any significant difference in primary cardiovascular outcome. The trial was even terminated early, due to higher occurrences of serious side effects, like bleeding and infection in the niacin treated group ${ }^{87}$.

Another method to raise HDL levels would be CETP inhibition, since CETP transfers cholesteryl esters from HDL to ApoB-containing lipoproteins. Although various animal studies showed conflicting results of the effects of CETP inhibition ${ }^{88,89}$, several promising inhibitors have been used in clinical trials. Thus far CETP inhibition as therapy seems to be quite challenging, since a trial using torcetrapib was terminated early because of significantly higher mortality rates in torcetrapib treated patients, whereas a trial using dalcetrapib was stopped due to futility ${ }^{90,91}$. Later it was shown that torcetrapib had unexpected off-target effects on the renin-angiotensin-aldosterone system, leading to hypertension and hypokalemia in patients ${ }^{92}$. More recently, a trial using anacetrapib did show significant increases in HDL levels without major side effect, though no beneficial effect on cardiovascular outcome was observed ${ }^{93,94}$. However, the small population size might have obscured any beneficial effects. Therefore currently the Randomized Evaluation of the Effects of Anacetrapib Through Lipid-modification (REVEAL) trial is started enrolling 30,000 patients. This study should give the definitive answer whether anacetrapib is indeed beneficial with regard to clinical outcome. Finally, evacetrapib is in clinical development and showed promising results already by beneficially modifying plasma lipid levels ${ }^{95}$. Currently, the phase III clinical trial, A Study of Evacetrapib in High-Risk Vascular Disease (ACCELERATE) is on-going. 
Fibrates can also inhibit CETP activity, next to increasing the synthesis of ApoA-I or ABCA1, via PPAR- $\alpha$ activation ${ }^{96}$. However, the main effect of fibrates, next to HDL raising, is the reduction of plasma TG levels, which was confirmed in a trial using gemfibrozil that indeed showed this lipid modification, resulting in significant reductions of cardiovascular outcome ${ }^{97}$. Additionally, a meta-analysis showed a $22 \%$ reduction of Ml risk, furthermore showing that fibrates are efficacious ${ }^{98}$. PPAR- $\alpha$ activation resulting in HDL raising is also mediated by statins ${ }^{99}$. However, the major effect of statins is the reduction of LDL by inhibiting 3-hydroxy-3-methylglutaryl coenzyme A (HMG CoA $)^{100}$. Currently, statins are widely used as the therapy of choice to reduce $L D L$ cholesterol and reduce cardiovascular risk.

The most direct way to elevate plasma HDL is by direct infusion. The Effect of rHDL on Atherosclerosis-Safety and Efficacy (ERASE) study is a small randomized clinical trial showing that infusion of reconstituted HDL significantly reduced atheroma volume ${ }^{101}$. Another clinical trial using HDL infusion used reconstituted $\mathrm{HDL}$ containing ApoA-I Milano, a naturally occurring mutant of ApoA-I, also showing significant reductions in atheroma volume ${ }^{102}$. Both studies clearly indicate beneficial effects of HDL/ApoA-I infusions, although much larger clinical studies are needed to assess its efficacy in reducing cardiovascular risk ${ }^{103}$. Also HDL mimetic peptides have already been well established in basic research, although not so elaborately yet in a clinical setting ${ }^{104}$. For example, in vitro it was shown that the peptide $5 \mathrm{~A}$ was effective in reducing VCAM-1 and ICAM-1 expression in human coronary artery endothelial cells ${ }^{105}$. The mimetic peptide ETC-642 was also shown to reduce vascular inflammation in vitro as well as in animals ${ }^{106}$. Another ApoA-I mimetic peptide, called 4F, was able to reduce atherosclerosis development in mice 107. Studies using mimetic peptide D-4F in humans showed that this peptide is safe and well tolerated, opening the doors for more elaborate clinical trials 108. Animal models show that hepatic ApoA-I overexpression also results in increased plasma HDL levels and a significant reduction in atherosclerosis development ${ }^{109}$. Therefore a compound called RVX-208 was developed, that specifically increases endogenous hepatic ApoA-I production. Unfortunately, a recent clinical trial (ASSURE trial) by Nicholls et al. showed that RVX-208 had no beneficial effects in patients (ESC press release 2013). 


\section{Current HDL hypothesis debate}

Several new developments, like many of the described clinical trials that did not show any effect on cardiovascular event rates, started a lively debate about the originally described HDL hypothesis and HDL raising therapeutic strategies. The HDL hypothesis recently also took a big hit by the meta-analysis of 39 clinical trials performed by Keene et al., showing that niacin, fibrates and CETP inhibitors did not reduce all-cause mortality or the incidence of $\mathrm{Ml}$ or stroke ${ }^{110}$. In addition to these clinical trial results, also genetic evidence shows inconsistencies as Voight et al. showed that polymorphisms exclusively altering HDL levels did not affect the risk of MI in mendelian randomisation analyses ${ }^{111}$. This debate makes $\mathrm{HDL}$ research more than ever an exciting and challenging field of interest. 


\section{Hypothesis and thesis outline}

In this thesis, various approaches are used to modulate vascular inflammation, focusing on both ADAMs and HDL, targeted in different cell-types.

Since ADAMs can shed various cytokines, chemokines and adhesion molecules which are all implicated in general inflammation, we hypothesize that they are critical players in atherosclerosis development. In chapter 2, a review is given on the role of ADAMs in angiogenesis, inflammation and atherosclerosis. Although many studies already show results implicating ADAMs in crucial mechanisms of atherosclerosis and we already showed an association between ADAM10 and human atherosclerosis progression, the causal role of ADAMs in atherosclerosis still remains unknown. Therefore, the first aim of this thesis is to determine the cell-type specific effects of different ADAMs on atherosclerosis development. In chapter 3, we study the role of ADAM8 in atherogenesis. We first confirm the already described association of ADAM8 with lesion development, by showing increased ADAM8 expression upon lesion progression in humans, especially in foamy macrophages. Hereafter, we investigate the effect of myeloid ADAM8 on atherosclerosis development in mice. In chapter 4 , we show the effect of another ADAM protease, ADAM10. Here, we investigate the causal role of myeloid ADAM10 in atherosclerosis development. In addition to myeloid cells, also endothelial cells play a crucial role in atherogenesis as these cells form the barrier between the lumen and vessel wall. Expression of adhesion molecules and secretion of chemokines from these cells is necessary for atherosclerosis development. As ADAM proteases can shed these molecules, we investigate in chapter 5 the effect of endothelial ADAM10 on atherosclerosis development. Atherosclerosis in this study is induced using a recently developed adeno-associated virus method ${ }^{112}$, resulting in LDLreceptor breakdown.

It is clear that HDL can have a major influence on atherosclerosis and cardiovascular disease. Recently, however, based on the disappointing clinical trials the debate started whether HDL is still a suitable therapeutic target to pursue. Many of these studies have investigated the HDL effects on a systemic pathogenesis and did not focus on the more local, cell specific HDL effects. Therefore the second aim of this thesis is to unravel the cell specific effects of HDL on smooth muscle cells (SMCs) and macrophages. 
Based on the already described anti-inflammatory and atheroprotective properties of HDL, we hypothesize that HDL will have anti-inflammatory effects on both cell-types by reducing cytokine and chemokine secretion. In chapter 6, we show the effects of HDL on SMC chemokine secretion and proliferation and unravel the mechanism behind these effects. Chapter 7 describes our findings regarding HDL effects on macrophage activation and the underlying mechanisms involved.

Chapter $\mathbf{8}$ discusses the most important findings of this thesis and puts them into a broader perspective of the current status of the field. Furthermore, future perspectives will be described. 


\section{References}

1. Dahlof B. Cardiovascular disease risk factors: Epidemiology and risk assessment. The American journal of cardiology. 2010;105:3A-9A

2. Lloyd-Jones DM. Cardiovascular risk prediction: Basic concepts, current status, and future directions. Circulation. 2010;121:1768-1777

3. Hansson GK. Inflammation, atherosclerosis, and coronary artery disease. The New England journal of medicine. 2005;352:1685-1695

4. Zarins CK, Giddens DP, Bharadvaj BK, Sottiurai VS, Mabon RF, Glagov S. Carotid bifurcation atherosclerosis. Quantitative correlation of plaque localization with flow velocity profiles and wall shear stress. Circulation research. 1983;53:502514

5. Williams KJ, Tabas I. The response-to-retention hypothesis of early atherogenesis. Arteriosclerosis, thrombosis, and vascular biology. 1995;15:551561

6. Tabas I, Williams KJ, Boren J. Subendothelial lipoprotein retention as the initiating process in atherosclerosis: Update and therapeutic implications. Circulation. 2007;116:1832-1844

7. Glass CK, Witztum JL. Atherosclerosis. The road ahead. Cell. 2001;104:503-516

8. Hansson GK, Hermansson A. The immune system in atherosclerosis. Nature immunology. 2011;12:204-212

9. Kunjathoor VV, Febbraio M, Podrez EA, Moore KJ, Andersson L, Koehn S, et al. Scavenger receptors class $\mathrm{a}-\mathrm{i} / \mathrm{ii}$ and $\operatorname{cd} 36$ are the principal receptors responsible for the uptake of modified low density lipoprotein leading to lipid loading in macrophages. The Journal of biological chemistry. 2002;277:4998249988

10. Pluddemann A, Neyen C, Gordon S. Macrophage scavenger receptors and hostderived ligands. Methods. 2007;43:207-217

11. Berliner JA, Territo MC, Sevanian A, Ramin S, Kim JA, Bamshad B, et al. Minimally modified low density lipoprotein stimulates monocyte endothelial interactions. The Journal of clinical investigation. 1990;85:1260-1266

12. Maxfield FR, Tabas I. Role of cholesterol and lipid organization in disease. Nature. 2005;438:612-621

13. Brown MS, Ho YK, Goldstein JL. The cholesteryl ester cycle in macrophage foam cells. Continual hydrolysis and re-esterification of cytoplasmic cholesteryl esters. The Journal of biological chemistry. 1980;255:9344-9352

14. Tall AR, Yvan-Charvet L, Terasaka N, Pagler T, Wang N. Hdl, abc transporters, and cholesterol efflux: Implications for the treatment of atherosclerosis. Cell 
metabolism. 2008;7:365-375

15. Wang X, Collins HL, Ranalletta M, Fuki IV, Billheimer JT, Rothblat GH, et al. Macrophage abca1 and abcg1, but not sr-bi, promote macrophage reverse cholesterol transport in vivo. The Journal of clinical investigation. 2007;117:22162224

16. Murphy AJ, Dragoljevic D, Tall AR. Cholesterol efflux pathways regulate myelopoiesis: A potential link to altered macrophage function in atherosclerosis. Frontiers in immunology. 2014;5:490

17. McLaren JE, Michael DR, Ashlin TG, Ramji DP. Cytokines, macrophage lipid metabolism and foam cells: Implications for cardiovascular disease therapy. Progress in lipid research. 2011;50:331-347

18. Dreymueller D, Pruessmeyer J, Groth E, Ludwig A. The role of adam-mediated shedding in vascular biology. European journal of cell biology. 2012;91:472-485

19. Woollard KJ, Geissmann F. Monocytes in atherosclerosis: Subsets and functions. Nature reviews. Cardiology. 2010;7:77-86

20. Swirski FK, Libby P, Aikawa E, Alcaide P, Luscinskas FW, Weissleder R, et al. Ly6chi monocytes dominate hypercholesterolemia-associated monocytosis and give rise to macrophages in atheromata. The Journal of clinical investigation. 2007;117:195-205

21. Moore KJ, Sheedy FJ, Fisher EA. Macrophages in atherosclerosis: A dynamic balance. Nature reviews. Immunology. 2013;13:709-721

22. Weber $\mathrm{C}$, Noels $\mathrm{H}$. Atherosclerosis: Current pathogenesis and therapeutic options. Nature medicine. 2011;17:1410-1422

23. Tacke F, Alvarez D, Kaplan TJ, Jakubzick C, Spanbroek R, Llodra J, et al. Monocyte subsets differentially employ ccr2, ccr5, and cx3cr1 to accumulate within atherosclerotic plaques. The Journal of clinical investigation. 2007;117:185-194

24. Combadiere C, Potteaux S, Rodero M, Simon T, Pezard A, Esposito B, et al. Combined inhibition of ccl2, cx3cr1, and ccr5 abrogates ly $6 \mathrm{c}(\mathrm{hi})$ and ly $6 \mathrm{c}(\mathrm{lo})$ monocytosis and almost abolishes atherosclerosis in hypercholesterolemic mice. Circulation. 2008;117:1649-1657

25. Weber C, Fraemohs L, Dejana E. The role of junctional adhesion molecules in vascular inflammation. Nature reviews. Immunology. 2007;7:467-477

26. Johnson JL, Newby AC. Macrophage heterogeneity in atherosclerotic plaques. Current opinion in lipidology. 2009;20:370-378

27. Shimada K. Immune system and atherosclerotic disease: Heterogeneity of leukocyte subsets participating in the pathogenesis of atherosclerosis. Circulation journal : official journal of the Japanese Circulation Society. 2009;73:9941001

28. Wolfs IM, Donners MM, de Winther MP. Differentiation factors and cytokines in 
the atherosclerotic plaque micro-environment as a trigger for macrophage polarisation. Thrombosis and haemostasis. 2011;106:763-771

29. Tabas I. Macrophage death and defective inflammation resolution in atherosclerosis. Nature reviews. Immunology. 2010;10:36-46

30. Tabas I, Ron D. Integrating the mechanisms of apoptosis induced by endoplasmic reticulum stress. Nature cell biology. 2011;13:184-190

31. Virmani R, Burke AP, Kolodgie FD, Farb A. Vulnerable plaque: The pathology of unstable coronary lesions. Journal of interventional cardiology. 2002;15:439-446

32. Moore KJ, Tabas I. Macrophages in the pathogenesis of atherosclerosis. Cell. 2011;145:341-355

33. Lusis AJ. Atherosclerosis. Nature. 2000;407:233-241

34. Boyle JJ, Weissberg PL, Bennett MR. Tumor necrosis factor-alpha promotes macrophage-induced vascular smooth muscle cell apoptosis by direct and autocrine mechanisms. Arteriosclerosis, thrombosis, and vascular biology. 2003;23:1553-1558

35. Donners MM, Wolfs IM, Olieslagers S, Mohammadi-Motahhari Z, Tchaikovski V, Heeneman $S$, et al. A disintegrin and metalloprotease 10 is a novel mediator of vascular endothelial growth factor-induced endothelial cell function in angiogenesis and is associated with atherosclerosis. Arteriosclerosis, thrombosis, and vascular biology. 2010;30:2188-2195

36. Garton KJ, Gough PJ, Philalay J, Wille PT, Blobel CP, Whitehead RH, et al. Stimulated shedding of vascular cell adhesion molecule 1 (vcam-1) is mediated by tumor necrosis factor-alpha-converting enzyme (adam 17). The Journal of biological chemistry. 2003;278:37459-37464

37. Koenen RR, Pruessmeyer J, Soehnlein O, Fraemohs L, Zernecke A, Schwarz N, et al. Regulated release and functional modulation of junctional adhesion molecule a by disintegrin metalloproteinases. Blood. 2009;113:4799-4809

38. Ludwig A, Weber C. Transmembrane chemokines: Versatile 'special agents' in vascular inflammation. Thrombosis and haemostasis. 2007;97:694-703

39. Tsakadze NL, Sithu SD, Sen U, English WR, Murphy G, D'Souza SE. Tumor necrosis factor-alpha-converting enzyme (tace/adam-17) mediates the ectodomain cleavage of intercellular adhesion molecule-1 (icam-1). The Journal of biological chemistry. 2006;281:3157-3164

40. Virmani R, Kolodgie FD, Burke AP, Finn AV, Gold HK, Tulenko TN, et al. Atherosclerotic plaque progression and vulnerability to rupture: Angiogenesis as a source of intraplaque hemorrhage. Arteriosclerosis, thrombosis, and vascular biology. 2005;25:2054-2061

41. Webb NR, Moore KJ. Macrophage-derived foam cells in atherosclerosis: Lessons from murine models and implications for therapy. Current drug targets. 
2007;8:1249-1263

42. Curtiss LK, Valenta DT, Hime NJ, Rye KA. What is so special about apolipoprotein ai in reverse cholesterol transport? Arteriosclerosis, thrombosis, and vascular biology. 2006;26:12-19

43. Emerging Risk Factors C, Di Angelantonio E, Sarwar N, Perry P, Kaptoge S, Ray $\mathrm{KK}$, et al. Major lipids, apolipoproteins, and risk of vascular disease. Jama. 2009;302:1993-2000

44. Pirillo A, Norata GD, Catapano AL. High-density lipoprotein subfractions--what the clinicians need to know. Cardiology. 2013;124:116-125

45. Camont L, Chapman MJ, Kontush A. Biological activities of hdl subpopulations and their relevance to cardiovascular disease. Trends in molecular medicine. 2011;17:594-603

46. Davidson WS, Thompson TB. The structure of apolipoprotein a-i in high density lipoproteins. The Journal of biological chemistry. 2007;282:22249-22253

47. Barter PJ. Hugh sinclair lecture: The regulation and remodelling of hdl by plasma factors. Atherosclerosis. Supplements. 2002;3:39-47

48. Masson D, Jiang XC, Lagrost L, Tall AR. The role of plasma lipid transfer proteins in lipoprotein metabolism and atherogenesis. Journal of lipid research. 2009;50 Suppl:S201-206

49. Settasatian N, Duong M, Curtiss LK, Ehnholm C, Jauhiainen M, Huuskonen J, et al. The mechanism of the remodeling of high density lipoproteins by phospholipid transfer protein. The Journal of biological chemistry. 2001;276:26898-26905

50. von Eckardstein A, Nofer JR, Assmann G. High density lipoproteins and arteriosclerosis. Role of cholesterol efflux and reverse cholesterol transport. Arteriosclerosis, thrombosis, and vascular biology. 2001;21:13-27

51. Cheung MC, Albers JJ. Characterization of lipoprotein particles isolated by immunoaffinity chromatography. Particles containing a-i and a-ii and particles containing a-i but no a-ii. The Journal of biological chemistry. 1984;259:1220112209

52. Huang Y, von Eckardstein A, Wu S, Maeda N, Assmann G. A plasma lipoprotein containing only apolipoprotein e and with gamma mobility on electrophoresis releases cholesterol from cells. Proceedings of the National Academy of Sciences of the United States of America. 1994;91:1834-1838

53. Christoffersen C, Dahlback B, Nielsen LB. Apolipoprotein m: Progress in understanding its regulation and metabolic functions. Scandinavian journal of clinical and laboratory investigation. 2006;66:631-637

54. Tian L, Fu M. The relationship between high density lipoprotein subclass profile and apolipoprotein concentrations. Journal of endocrinological investigation. 2011;34:461-472 
55. Kingwell BA, Chapman MJ, Kontush A, Miller NE. Hdl-targeted therapies: Progress, failures and future. Nature reviews. Drug discovery. 2014;13:445-464

56. Kannel WB, Dawber TR, Friedman GD, Glennon WE, McNamara PM. Risk factors in coronary heart disease. An evaluation of several serum lipids as predictors of coronary heart disease; the framingham study. Annals of internal medicine. 1964;61:888-899

57. Badimon JJ, Badimon L, Fuster V. Regression of atherosclerotic lesions by high density lipoprotein plasma fraction in the cholesterol-fed rabbit. The Journal of clinical investigation. 1990;85:1234-1241

58. Rubin EM, Krauss RM, Spangler EA, Verstuyft JG, Clift SM. Inhibition of early atherogenesis in transgenic mice by human apolipoprotein ai. Nature. 1991;353:265-267

59. Miller NE, Thelle DS, Forde OH, Mjos OD. The tromso heart-study. High-density lipoprotein and coronary heart-disease: A prospective case-control study. Lancet. 1977; 1:965-968

60. Rye KA, Barter PJ. Cardioprotective functions of hdls. Journal of lipid research. 2014;55:168-179

61. Favari E, Calabresi L, Adorni MP, Jessup W, Simonelli S, Franceschini G, et al. Small discoidal pre-beta1 hdl particles are efficient acceptors of cell cholesterol via abca1 and abcg1. Biochemistry. 2009;48:11067-11074

62. Yokoyama S. Abca1 and biogenesis of hdl. Journal of atherosclerosis and thrombosis. 2006;13:1-15

63. Rothblat GH, Phillips MC. High-density lipoprotein heterogeneity and function in reverse cholesterol transport. Current opinion in lipidology. 2010;21:229-238

64. Ji Y, Jian B, Wang N, Sun Y, Moya ML, Phillips MC, et al. Scavenger receptor bi promotes high density lipoprotein-mediated cellular cholesterol efflux. The Journal of biological chemistry. 1997;272:20982-20985

65. Adorni MP, Zimetti F, Billheimer JT, Wang N, Rader DJ, Phillips MC, et al. The roles of different pathways in the release of cholesterol from macrophages. Journal of lipid research. 2007;48:2453-2462

66. Kontush A, Chantepie S, Chapman MJ. Small, dense hdl particles exert potent protection of atherogenic Idl against oxidative stress. Arteriosclerosis, thrombosis, and vascular biology. 2003;23:1881-1888

67. Mackness MI, Durrington PN, Mackness B. The role of paraoxonase 1 activity in cardiovascular disease: Potential for therapeutic intervention. American journal of cardiovascular drugs : drugs, devices, and other interventions. 2004;4:211-217

68. Christison JK, Rye KA, Stocker R. Exchange of oxidized cholesteryl linoleate between Idl and hdl mediated by cholesteryl ester transfer protein. Journal of lipid research. 1995;36:2017-2026 
69. Zerrad-Saadi A, Therond P, Chantepie S, Couturier M, Rye KA, Chapman MJ, et al. Hdl3-mediated inactivation of Idl-associated phospholipid hydroperoxides is determined by the redox status of apolipoprotein a-i and hdl particle surface lipid rigidity: Relevance to inflammation and atherogenesis. Arteriosclerosis, thrombosis, and vascular biology. 2009;29:2169-2175

70. Cockerill GW, Rye KA, Gamble JR, Vadas MA, Barter PJ. High-density lipoproteins inhibit cytokine-induced expression of endothelial cell adhesion molecules. Arteriosclerosis, thrombosis, and vascular biology. 1995;15:1987-1994

71. Bursill CA, Castro ML, Beattie DT, Nakhla S, van der Vorst E, Heather AK, et al. High-density lipoproteins suppress chemokines and chemokine receptors in vitro and in vivo. Arteriosclerosis, thrombosis, and vascular biology. 2010;30:17731778

72. Nicholls SJ, Cutri B, Worthley SG, Kee P, Rye KA, Bao S, et al. Impact of short-term administration of high-density lipoproteins and atorvastatin on atherosclerosis in rabbits. Arteriosclerosis, thrombosis, and vascular biology. 2005;25:2416-2421

73. Nicholls SJ, Dusting GJ, Cutri B, Bao S, Drummond GR, Rye KA, et al. Reconstituted high-density lipoproteins inhibit the acute pro-oxidant and proinflammatory vascular changes induced by a periarterial collar in normocholesterolemic rabbits. Circulation. 2005;111:1543-1550

74. Gordon SM, Hofmann S, Askew DS, Davidson WS. High density lipoprotein: It's not just about lipid transport anymore. Trends in endocrinology and metabolism: TEM. 2011;22:9-15

75. Nofer JR, Brodde MF, Kehrel BE. High-density lipoproteins, platelets and the pathogenesis of atherosclerosis. Clinical and experimental pharmacology \& physiology. 2010;37:726-735

76. Mineo C, Deguchi H, Griffin JH, Shaul PW. Endothelial and antithrombotic actions of hdl. Circulation research. 2006;98:1352-1364

77. Tso C, Martinic G, Fan WH, Rogers C, Rye KA, Barter PJ. High-density lipoproteins enhance progenitor-mediated endothelium repair in mice. Arteriosclerosis, thrombosis, and vascular biology. 2006;26:1144-1149

78. Yvan-Charvet L, Pagler T, Gautier EL, Avagyan S, Siry RL, Han S, et al. Atp-binding cassette transporters and hdl suppress hematopoietic stem cell proliferation. Science. 2010;328:1689-1693

79. Kodama S, Tanaka S, Saito K, Shu M, Sone Y, Onitake F, et al. Effect of aerobic exercise training on serum levels of high-density lipoprotein cholesterol: A meta-analysis. Archives of internal medicine. 2007;167:999-1008

80. Carlson LA. Nicotinic acid: The broad-spectrum lipid drug. A 50th anniversary review. Journal of internal medicine. 2005;258:94-114

81. Kamanna VS, Ganji SH, Kashyap ML. Recent advances in niacin and lipid 
metabolism. Current opinion in lipidology. 2013;24:239-245

82. van der Hoorn JW, de Haan W, Berbee JF, Havekes LM, Jukema JW, Rensen PC, et al. Niacin increases hdl by reducing hepatic expression and plasma levels of cholesteryl ester transfer protein in apoe*3leiden.Cetp mice. Arteriosclerosis, thrombosis, and vascular biology. 2008;28:2016-2022

83. Investigators A-H, Boden WE, Probstfield JL, Anderson T, Chaitman BR, DesvignesNickens $P$, et al. Niacin in patients with low hdl cholesterol levels receiving intensive statin therapy. The New England journal of medicine. 2011;365:22552267

84. Taylor AJ, Lee HJ, Sullenberger LE. The effect of $\mathbf{2 4}$ months of combination statin and extended-release niacin on carotid intima-media thickness: Arbiter 3. Current medical research and opinion. 2006;22:2243-2250

85. Taylor AJ, Sullenberger LE, Lee HJ, Lee JK, Grace KA. Arterial biology for the investigation of the treatment effects of reducing cholesterol (arbiter) 2: A double-blind, placebo-controlled study of extended-release niacin on atherosclerosis progression in secondary prevention patients treated with statins. Circulation. 2004;110:3512-3517

86. Taylor AJ, Villines TC, Stanek EJ, Devine PJ, Griffen L, Miller M, et al. Extendedrelease niacin or ezetimibe and carotid intima-media thickness. The New England journal of medicine. 2009;361:2113-2122

87. Group HTC. Hps2-thrive randomized placebo-controlled trial in 25673 high-risk patients of er niacin/laropiprant: Trial design, pre-specified muscle and liver outcomes, and reasons for stopping study treatment. European heart journal. 2013;34:1279-1291

88. Huang Z, Inazu A, Nohara A, Higashikata T, Mabuchi H. Cholesteryl ester transfer protein inhibitor (jtt-705) and the development of atherosclerosis in rabbits with severe hypercholesterolaemia. Clinical science. 2002;103:587-594

89. Okamoto H, Yonemori F, Wakitani K, Minowa T, Maeda K, Shinkai H. A cholesteryl ester transfer protein inhibitor attenuates atherosclerosis in rabbits. Nature. 2000;406:203-207

90. Barter PJ, Caulfield M, Eriksson M, Grundy SM, Kastelein JJ, Komajda M, et al. Effects of torcetrapib in patients at high risk for coronary events. The New England journal of medicine. 2007;357:2109-2122

91. Schwartz GG, Olsson AG, Abt M, Ballantyne CM, Barter PJ, Brumm J, et al. Effects of dalcetrapib in patients with a recent acute coronary syndrome. The New England journal of medicine. 2012;367:2089-2099

92. Vergeer M, Stroes ES. The pharmacology and off-target effects of some cholesterol ester transfer protein inhibitors. The American journal of cardiology. 2009;104:32E-38E 
93. Cannon CP, Dansky HM, Davidson M, Gotto AM, Jr., Brinton EA, Gould AL, et al. Design of the define trial: Determining the efficacy and tolerability of cetp inhibition with anacetrapib. American heart journal. 2009;158:513-519 e513

94. Cannon CP, Shah S, Dansky HM, Davidson M, Brinton EA, Gotto AM, et al. Safety of anacetrapib in patients with or at high risk for coronary heart disease. The New England journal of medicine. 2010;363:2406-2415

95. Nicholls SJ, Brewer HB, Kastelein JJ, Krueger KA, Wang MD, Shao M, et al. Effects of the cetp inhibitor evacetrapib administered as monotherapy or in combination with statins on hdl and Idl cholesterol: A randomized controlled trial. Jama. 2011;306:2099-2109

96. Staels B, Dallongeville J, Auwerx J, Schoonjans K, Leitersdorf E, Fruchart JC. Mechanism of action of fibrates on lipid and lipoprotein metabolism. Circulation. 1998;98:2088-2093

97. Robins SJ, Collins D, Wittes JT, Papademetriou V, Deedwania PC, Schaefer EJ, et al. Relation of gemfibrozil treatment and lipid levels with major coronary events: Va-hit: A randomized controlled trial. Jama. 2001;285:1585-1591

98. Saha SA, Kizhakepunnur LG, Bahekar A, Arora RR. The role of fibrates in the prevention of cardiovascular disease--a pooled meta-analysis of longterm randomized placebo-controlled clinical trials. American heart journal. 2007; 154:943-953

99. Martin G, Duez H, Blanquart C, Berezowski V, Poulain P, Fruchart JC, et al. Statininduced inhibition of the rho-signaling pathway activates pparalpha and induces hdl apoa-i. The Journal of clinical investigation. 2001;107:1423-1432

100. Nicholls SJ, Tuzcu EM, Sipahi I, Grasso AW, Schoenhagen P, Hu T, et al. Statins, highdensity lipoprotein cholesterol, and regression of coronary atherosclerosis. Jama. 2007;297:499-508

101. Tardif JC, Gregoire J, L'Allier PL, Ibrahim R, Lesperance J, Heinonen TM, et al. Effects of reconstituted high-density lipoprotein infusions on coronary atherosclerosis: A randomized controlled trial. Jama. 2007;297:1675-1682

102. Nissen SE, Tsunoda T, Tuzcu EM, Schoenhagen P, Cooper CJ, Yasin M, et al. Effect of recombinant apoa-i milano on coronary atherosclerosis in patients with acute coronary syndromes: A randomized controlled trial. Jama. 2003;290:22922300

103. Tuteja S, Rader DJ. High-density lipoproteins in the prevention of cardiovascular disease: Changing the paradigm. Clinical pharmacology and therapeutics. 2014;96:48-56

104. Navab M, Reddy ST, Van Lenten BJ, Fogelman AM. Hdl and cardiovascular disease: Atherogenic and atheroprotective mechanisms. Nature reviews. Cardiology. 2011;8:222-232 
105. Tabet F, Remaley AT, Segaliny Al, Millet J, Yan L, Nakhla S, et al. The 5a apolipoprotein a-i mimetic peptide displays antiinflammatory and antioxidant properties in vivo and in vitro. Arteriosclerosis, thrombosis, and vascular biology. 2010;30:246-252

106. Di Bartolo BA, Nicholls SJ, Bao S, Rye KA, Heather AK, Barter PJ, et al. The apolipoprotein a-i mimetic peptide etc-642 exhibits anti-inflammatory properties that are comparable to high density lipoproteins. Atherosclerosis. $2011 ; 217: 395-400$

107. Wool GD, Cabana VG, Lukens J, Shaw PX, Binder CJ, Witztum JL, et al. 4f peptide reduces nascent atherosclerosis and induces natural antibody production in apolipoprotein e-null mice. FASEB journal : official publication of the Federation of American Societies for Experimental Biology. 2011;25:290-300

108. Bloedon LT, Dunbar R, Duffy D, Pinell-Salles P, Norris R, DeGroot BJ, et al. Safety, pharmacokinetics, and pharmacodynamics of oral apoa-i mimetic peptide $\mathbf{d - 4 f}$ in high-risk cardiovascular patients. Journal of lipid research. 2008;49:1344-1352

109. Tangirala RK, Tsukamoto K, Chun SH, Usher D, Pure E, Rader DJ. Regression of atherosclerosis induced by liver-directed gene transfer of apolipoprotein a-i in mice. Circulation. 1999;100:1816-1822

110. Keene D, Price C, Shun-Shin MJ, Francis DP. Effect on cardiovascular risk of high density lipoprotein targeted drug treatments niacin, fibrates, and cetp inhibitors: Meta-analysis of randomised controlled trials including 117,411 patients. Bmj. 2014;349:g4379

111. Voight BF, Peloso GM, Orho-Melander M, Frikke-Schmidt R, Barbalic M, Jensen MK, et al. Plasma hdl cholesterol and risk of myocardial infarction: A mendelian randomisation study. Lancet. 2012;380:572-580

112. Bjorklund MM, Hollensen AK, Hagensen MK, Dagnaes-Hansen F, Christoffersen C, Mikkelsen JG, et al. Induction of atherosclerosis in mice and hamsters without germline genetic engineering. Circulation research. 2014;114:1684-1689 


\section{Chapter 2}

\section{A Disintegrin And Metalloproteases: Molecular scissors in angiogenesis, inflammation and atherosclerosis}

Emiel P.C. van der Vorst", Anke A. Keijbeck ${ }^{*}$, Menno P.J. de Winther, Marjo M.P.C. Donners

${ }^{*}$ Authors contributed equally

Atherosclerosis, 2012 Oct;224(2):302-8 


\section{Abstract}

A Disintegrin And Metalloproteases (ADAMs) are enzymes that cleave (shed) the extracellular domains of various cell surface molecules, e.g. adhesion molecules, cytokine/chemokine and growth factor receptors, thereby releasing soluble molecules that can exert agonistic or antagonistic functions or serve as biomarkers. By functioning as such molecular scissors, ADAM proteases have been implicated in various diseases, e.g. cancer, and their role in cardiovascular diseases is now emerging. This review will focus on the role of ADAM proteases in molecular mechanisms of angiogenesis and inflammation in relation to atherosclerosis. Besides a concise overview of the current state and recent advances of this research area, we will discuss key questions about redundancy, specificity and regulation of ADAM proteases and emphasize the importance of confirmation of in vitro findings in in vivo models. 


\section{Introduction}

To date 40 ADAM family members have been identified in the mammalian genome, typically consisting of conserved and characteristic protein domains (Fig. 1) ${ }^{1}$. Only 12 ADAM genes encode for proteolytically active proteases, i.e. ADAM8, 9, 10, 12, 15, 17, 19, 20, 21, 28, 30 and $33^{1,2}$, of which ADAM10 and 17 are the best studied family members ${ }^{3}$. In this review we will specifically focus on the ADAMs with sheddase activities, i.e. the ADAMs mediating proteolytic cleavage of transmembrane proteins near the plasma membrane releasing the extracellular part (ectodomains) (Fig. 1). This process called ectodomain shedding affects various surface proteins, such as growth factors, adhesion molecules, cytokines and cytokine receptors ${ }^{2}$. Cleavage of transmembrane proteins by ADAMs can be followed by a process known as regulated intramembrane proteolysis (RIP), leading to the intracellular release of signaling molecules (Fig. 1) ${ }^{2}$. In this review, we focus on the roles of ADAM sheddases and regulation of their activities in angiogenesis and inflammatory processes in relation to atherosclerosis.

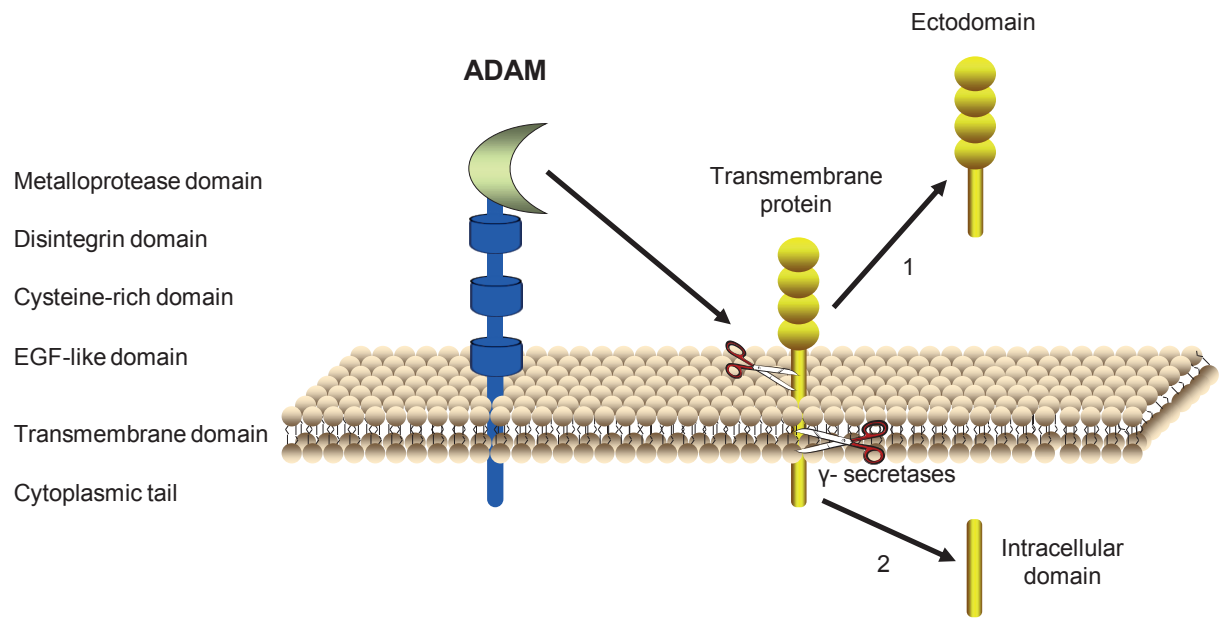

Figure 1. General domain structure and function of active ADAMs. Process 1: ectodomain shedding. Process 2: regulated intramembrane proteolysis (RIP), i.e. initial juxtamembrane cleavage by ADAMs and subsequent intramembrane cleavage by $\mathrm{\gamma}$-secretases. 


\section{Regulation of ADAMs shedding activities}

Regulation of ADAM activity occurs at various levels including transcriptional control, post-translational processing, localization within the plasma membrane and substrate regulation ${ }^{3}$. ADAM proteases are produced as latent enzymes and activated by removal of the prodomain ${ }^{1}$. Inhibition can be regulated by Tissue Inhibitors of Metalloproteases (TIMPs), e.g. TIMP1 blocks ADAM10, whereas TIMP3 inhibits both ADAM10 and ADAM17 ${ }^{4}$. ADAM mediated shedding can occur both constitutively and in response to a variety of stimuli (inducible shedding) ${ }^{5}$. ADAM10-dependent shedding for instance can be induced by stimulating intracellular calcium signaling, while ADAM17 activity is induced by protein kinase $\mathrm{C}$ activation ${ }^{6}$. Various inflammatory, angiogenic and atherogenic stimuli, e.g. cytokines (TNF- $\alpha$, IFN- ), growth factors (VEGF, PDGF) and oxidized low-density lipoproteins (oxLDL) $)^{7,8}$ may regulate ADAMs activity, but specificity, temporal and spatial mechanisms as well as downstream effects on atherosclerosis remain to be elucidated.

ADAM-mediated shedding does not depend on a specific consensus sequence. Recently, lipid rafts were suggested as regions that could regulate shedding activity by bringing enzyme and substrate together ${ }^{4}$. Lipid rafts are cholesterol-and spingolipid-enriched microdomains within the cell membrane that serve as organizing centers for the assembly of receptors and signaling molecules, e.g. leading to signaling initiation ${ }^{9}$. Lipid rafts are increased under atherosclerotic/hyperlipidemic conditions and can be decreased by cholesterol depletion, e.g. by high-density lipoproteins (HDL) or statins ${ }^{10}$. Until now, only few studies focused on lipid rafts as regulatory mechanism for ADAM activity. Tellier et al. ${ }^{9}$ showed that mature (i.e. active) ADAM17 in endothelial cells (ECs) and fibroblasts is highly concentrated in these rafts. The importance of lipid rafts in the regulation of ADAM17 substrate cleavage is evidenced by the fact that lipid raft disruption (by cholesterol depletion, e.g. by HDL) increases cleavage of TNF- $\alpha$ in these cells ${ }^{9}$. Additionally, more than half of the identified ADAM17 substrates are localized in lipid rafts. Besides ADAM17 cleavage, also cleavage of CD44 by ADAM10 in tumor cells has been shown to be located in lipid rafts ${ }^{11}$. However, the number of studies so far are limited and how exactly lipid rafts are involved in regulating ADAMsmediated shedding upon various stimuli in specific atherosclerotic processes is not known so far. 
Many substrates of ADAMs have already been identified and the list of substrates is still increasing (Table 1 and 2). Especially ADAM10 and ADAM17 are closely related proteases and share many substrates, suggesting redundancy of these proteases. Indeed Le Gall et al. showed that ADAM10 can process substrates of ADAM17, but only when this primary sheddase is absent ${ }^{5}$. Importantly a role for ADAM10 as a secondary sheddase has only been shown in vitro and functional redundancy in vivo remains to be demonstrated. Recently, a study by Tang et al. ${ }^{12}$ showed that multiple adhesion molecules (e.g. CD44, ICAM-1 and L-selectin) are shed by ADAM17 in vitro, although only a limited number of substrates are confirmed to be shed in vivo. In addition, the embryonic lethality of both ADAM10--- and ADAM17-- mice is a clear indication of the lack of in vivo redundancy between these proteases. Indeed, it has been shown that ADAM17 cannot substitute as sheddase for substrates of ADAM10 in ADAM10-1- cells ${ }^{5,6}$. Future research should further extend our knowledge on mechanisms regulating ADAMs activity and specificity in vivo. 
Table 1. ADAM proteases involved in (plaque) angiogenesis

\begin{tabular}{|c|c|c|c|}
\hline ADAM & $\begin{array}{c}\text { In vitro } \\
\text { substrates * }\end{array}$ & $\begin{array}{c}\text { In vitro model or } \\
\text { assay }\end{array}$ & In vivo \\
\hline ADAM8 & Not identified & Not investigated & $\begin{array}{l}\text { ADAM8-/- } \uparrow \text { oxygen- } \\
\text { induced retinal re- } \\
\text { vascularization }{ }^{13}\end{array}$ \\
\hline ADAM9 & $\begin{array}{l}\text { CD40 }{ }^{14} \\
\text { Tie-2 }^{14} \\
\text { VE-cadherin }{ }^{14} \\
\text { ProHB-EGF }{ }^{2}\end{array}$ & Not investigated & $\begin{array}{l}\text { ADAM9-/- } \downarrow \text { oxygen- } \\
\text { induced pathological } \\
\text { neovascularization }{ }^{14} \\
\text { ADAM9 overexpression } \\
\uparrow \text { shedding of CD40, Tie-2 } \\
\text { and VE-cadherin }{ }^{14}\end{array}$ \\
\hline ADAM10 & $\begin{array}{l}\text { VEGFR2 }^{15} \\
\text { NRP-1 }^{16} \\
\text { JAM-A }^{17} \\
\text { VE-cadherin }^{15} \\
\text { Notch }^{18}\end{array}$ & $\begin{array}{l}\downarrow \text { endothelial tube } \\
\text { formation in } 2 \text {-D } \\
\text { matrigel (own } \\
\text { unpublished data) } \\
\text { ADAM10 inhibition } \\
\downarrow \text { EC migration and } \\
\text { chemotaxis }^{15}\end{array}$ & $\begin{array}{l}\text { ADAM10-/- lethal due to } \\
\text { defects in cardiovascular } \\
\text { system and } \\
\text { vasculogenesis } 2,19 \\
\text { ADAM10-Tie2-Cre show } \\
\text { vascular abnormalities, } \\
\text { defects in various organs } \\
\text { and } \downarrow \text { pathological } \\
\text { neovascularization } 20\end{array}$ \\
\hline ADAM15 & Pro HB-EGF ${ }^{2}$ & $\begin{array}{l}\text { ADAM-15 } \\
\text { recombinant } \\
\text { disintegrin domain } \\
\downarrow \text { capillary-like } \\
\text { structure formation } \\
\text { ECs in a } 3-D \text { fibrin } \\
\text { gel }{ }^{21}\end{array}$ & $\begin{array}{l}\text { ADAM15-/- } \downarrow \text { angiogenic } \\
\text { response in murine } \\
\text { retinopathy of prematurity } \\
\text { model } 22\end{array}$ \\
\hline ADAM17 & $\begin{array}{l}\text { VEGFR2 }{ }^{16} \\
\text { Tie-2 }{ }^{11} \\
\text { JAM-A }{ }^{17} \\
\text { Notch }{ }^{18}\end{array}$ & $\begin{array}{l}\text { ADAM17 inhibition } \\
\downarrow \text { EC proliferation, } \\
\text { destabilization of } \\
\text { capillary networks } \\
\text { and } \downarrow \text { endothelial } \\
\text { invasion in } 3-D \\
\text { matrigel assay } 23\end{array}$ & $\begin{array}{l}\text { ADAM17 Tie2-Cre mice } \\
\text { show larger avascular } \\
\text { area in oxygen-induced } \\
\text { retinopathy }{ }^{24}\end{array}$ \\
\hline
\end{tabular}

*relevant to angiogenesis 


\section{Role of ADAMs in atherosclerosis}

ADAM mediated cleavage of specific substrates has been associated with (protective mechanisms in) various diseases, such as Alzheimer disease, rheumatoid arthritis and cancer ${ }^{3}$. Atherosclerosis is a chronic inflammatory disease of the vessel wall. Considering the role of ADAMs in other inflammatory diseases and angiogenesis as described below, they most likely play an important role in cardiovascular diseases.

\section{Neovessel formation}

Angiogenesis is the formation of new blood vessels in both physiological processes, like wound healing, and pathophysiological processes including cancer and atherosclerosis. Several ADAM proteases are associated with angiogenesis (Table 1). Recently, we identified ADAM10 as a novel binding partner and in vitro sheddase of the major angiogenic receptor Vascular Endothelial Growth Factor Receptor VEGFR2 ${ }^{15}$. VEGF stimulation of ECs increases ADAM10 expression and activity, which mediates (VEGF-induced) EC functions such as migration, chemotaxis and EC permeability. Similarly, VEGF was shown to activate ADAM17 shedding, also triggering cleavage of VEGFR2 (e.g. as a negative feedback loop) and other ADAM17 substrates such as TNF- $\alpha$ and Tie-2, whereas ADAM10 was shown to be responsible for cleavage of the co-receptor neuropilin- ${ }^{16}$. In addition, ADAM10 and ADAM17 can cleave endothelial junctional adhesion molecules (JAMs), such as JAM-A 17 and vascular endothelial cadherin (VE-cadherin) ${ }^{15}$, thereby increasing vascular permeability and EC migration, a crucial step in angiogenesis.

Other important substrates of ADAMs involved in angiogenesis are molecules from the Notch signaling pathway ${ }^{25}$, e.g. Notch1 and Delta-like 4 are involved in physiological and tumor angiogenesis, especially in vessel sprouting 25. Although both ADAM10 and ADAM17 have been implicated in Notch processing, ADAM10 appears to be the major Notch-processing enzyme in vivo, as is evidenced by the phenotypic resemblance of ADAM10 knockout $\left(\right.$ ADAM10 ${ }^{-/}$) and Notch1 ${ }^{-/-} /$Notch $4^{-/}$mice (described below) ${ }^{19}$.

Various studies indeed demonstrated an important role for ADAMs in angiogenic processes in vitro. ADAM17-deficient ECs showed reduced proliferation due to decreased activation of several growth factors, e.g. VEGF, and cytokine signaling pathways ${ }^{23}$. Furthermore, ADAM17 inhibition 
destabilizes the capillary networks and decreased EC invasion in a 3-D matrigel ${ }^{23,26}$, whereas overexpression of ADAM17 results in a far greater number of endothelial sprouts ${ }^{26}$. Besides ADAM17, ADAM10 was also found to reduce endothelial tube formation in a 2-D matrigel assay (own unpublished data).

Several studies have shown a role of ADAMs in angiogenesis in vivo by using knockout mice. ADAM8 $8^{-1-}$ mice ${ }^{13}$ display increased, whereas ADAM9-- and ADAM15 ${ }^{-1-}$ mice ${ }^{14,22}$ show decreased retinal re-vascularization in a mouse model of oxygen-induced retinopathy pathological neovascularization. Total ADAM10-- mice die already at 9.5 days of embryogenesis with multiple defects in both cardiovascular system and vasculogenesis, including malformation of the vessels ${ }^{2}$. Phenotypically these mice resemble the Notch $1^{-1 / N o t c h} 4^{--}$mice, again indicating the crucial role of ADAM10 in Notch signaling and (neo)vessel formation in vivo ${ }^{19}$. Cell-type specific deletion of ADAM10 in ECs (ADAM10-Tie2-Cre mice) induce vascular abnormalities and decreased pathological neovascularization, along with various defects in other organs ${ }^{20}$. Similarly, ADAM17-Tie2-cre mice show reduced pathological neovascularization in oxygen-induced retinopathy ${ }^{24}$.

Angiogenesis has been associated with atherosclerotic lesion growth and decreased plaque stability ${ }^{27}$. Although the previously described studies do not specifically investigate plaque angiogenesis, it is very likely that ADAMs also play a major role in plaque angiogenesis. Interestingly, we found high in vivo ADAM10 expression associated with plaque angiogenesis in human atherosclerosis ${ }^{15}$. Moreover, plaque microvessels are considered to be immature and 'leaky', i.e. displaying increased vessel permeability facilitating inflammatory cell entry ${ }^{27}$. Considering the importance of ADAM proteases in cleaving cell-cell adhesion contacts controlling vascular permeability as described above, it is tempting to speculate that ADAMs could modulate plaque angiogenesis and microvessel functionality thereby influencing plaque inflammatory status and stability. The causal role of ADAM proteases in plaque angiogenesis and atherosclerotic plaque growth and stability, however, remains to be determined. 


\section{Recruitment of inflammatory cells}

Atherosclerosis is a chronic inflammatory disease, characterized by the accumulation of lipids and inflammatory cells in the vessel wall. Besides plaque angiogenesis, the balance between pro-inflammatory and antiinflammatory cytokines highly influences progression and stability of atherosclerotic lesions and thereby determines clinical outcome ${ }^{27}$.

ADAM proteases have been shown to have various effects on inflammatory mediators, some of which are crucial in the pathogenesis of atherosclerosis (Table 2). Leukocyte recruitment to the injured vessel wall is a critical step in both initiation and progression of atherosclerosis and can be modulated by ADAMs by cleavage of various adhesion molecules. As previously mentioned, ADAM-mediated cleavage of JAM-A and VE-cadherin ${ }^{15,17}$ increases vascular permeability and leukocyte transmigration. $\mathrm{CX}_{3} \mathrm{CL} 1$ (fractalkine) and CXCL16 are two transmembrane chemokines involved in atherosclerosis that act as adhesion molecules in their membrane-bound form, whereas in soluble form they serve as chemotactic factors for leukocytes ${ }^{28}$. Moreover, CXCL16 can act as a scavenger receptor for oxLDL ${ }^{28,29}$. The role of ADAMs 8, 10 and 17 in cleavage of these chemokines has been comprehensively reviewed by Ludwig et al ${ }^{28}$. Two other essential adhesion molecules involved in leukocyte adhesion to the vessel wall, VCAM-I and ICAM-I can also be cleaved by ADAM17 ${ }^{30,31}$. Besides modulating leukocyte adhesion, the soluble forms of VCAM-I and ICAM-I may be used as biomarkers for atherosclerosis ${ }^{32}$. The role of ADAMs in leukocyte recruitment in vivo has indeed been confirmed by Tang et al. who showed that ADAM17-- neutrophils roll slower and adhere more to the vessel wall than wild-type neutrophils ${ }^{12}$. Furthermore, hematopoietic ADAM17-deficient (ADAM17-vav-cre ${ }^{-/}$) mice have increased neutrophil infiltration into the peritoneal cavity ${ }^{33}$. The role of ADAMsmediated cleavage of adhesion molecules in leukocyte recruitment to the atherosclerotic lesions, however, has not been determined so far. 
Table 2 ADAM proteases involved in inflammation and atherosclerosis

\begin{tabular}{|c|c|c|c|}
\hline ADAM & $\begin{array}{c}\text { In vitro } \\
\text { substrates }\end{array}$ & $\begin{array}{c}\text { In vitro model or } \\
\text { assay }\end{array}$ & In vivo \\
\hline ADAM8 & $\begin{array}{l}\text { L-selectin }^{2} \\
\text { TNFRI }^{51} \\
\text { CX }_{3}{ }_{3}{ }{ }^{52}\end{array}$ & Not investigated & Not investigated \\
\hline ADAM9 & $\begin{array}{l}\text { TNF }^{37} \\
\text { TNFRII }\end{array}$ & Not investigated & $\begin{array}{l}\text { ADAM9 is expressed in } \\
\text { human atherosclerotic } \\
\text { plaques co-localizing with } \\
\text { macrophages }{ }^{43}\end{array}$ \\
\hline ADAM10 & $\begin{array}{l}\text { TNF }^{37} \\
\text { IL-6R }^{42} \\
\text { CD44 }^{53} \\
\text { CX }_{3} \mathrm{CL}^{28} \\
\text { CXCL16 } \\
\end{array}$ & $\begin{array}{l}\text { ADAM10 inhibition } \\
\uparrow \text { monocyte adhesion } \\
\text { to CX3CL1 } \\
\text { expressing EC }{ }^{54} \\
\text { ADAM10 inhibition } \\
\downarrow \text { CX3CR1-mediated } \\
\text { leukocyte } \\
\text { transmigration } \\
\text { through CX3CL1- } \\
\text { expressing EC } \\
\text { and leukocyte } \\
\text { transmigration } \\
\text { through EC }{ }^{55}\end{array}$ & $\begin{array}{l}\text { ADAM10 is expressed in } \\
\text { macrophages/foam cells } \\
\text { and ECs in human } \\
\text { atherosclerotic lesions and } \\
\uparrow \text { with disease progression } \\
15\end{array}$ \\
\hline ADAM15 & & $\begin{array}{l}\text { ADAM15 } \\
\text { overexpression in } \\
\text { ECs } \uparrow \text { platelet } \\
\text { adhesion }{ }^{21} \\
\text { ADAM15 } \\
\text { recombinant } \\
\text { disintegrin domain } \\
\downarrow \text { EC adhesion } \\
\text { ADAM-15 } \downarrow \text { PDGF } \\
\text { induced Airway SMC } \\
\text { migration }{ }^{46}\end{array}$ & $\begin{array}{l}\text { ADAM15 is expressed in } \\
\text { human atherosclerotic } \\
\text { plaques co-localizing with } \\
\text { macrophages }{ }^{43} \\
\text { Overexpression of active, } \\
\text { but not inactive, ADAM15 } \\
\text { atherosclerosis in rabbits }{ }^{48}\end{array}$ \\
\hline
\end{tabular}




\begin{tabular}{|c|c|c|c|}
\hline ADAM17 & $\begin{array}{l}\text { TNF, TNFRI } \\
\text { and TNFRII } \\
37 \\
\text { MCSF- } \\
\text { receptor }^{34} \\
\text { CD163 }{ }^{41} \\
\text { IL-6R }{ }^{42} \\
\text { CD44 }{ }^{53} \\
\text { VCAM-I } 30 \\
\text { ICAM-I }^{31} \\
\text { CX }{ }_{3} \text { CL1 and } \\
\text { CXCL16 }{ }^{28} \\
\text { L-Selectin, } \\
\text { CD30, CD40, } \\
\text { IL-1RII, IL- } \\
\text { 15R } 56\end{array}$ & $\begin{array}{l}\text { ADAM17 inhibition } \\
\downarrow \text { CX3CR1-mediated } \\
\text { leukocyte } \\
\text { transmigration } \\
\text { through CX3CL1- } \\
\text { expressing EC }{ }^{55}\end{array}$ & $\begin{array}{l}\text { Reduced pro-inflammatory } \\
\text { cytokine release in } \\
\text { ADAM17-LysMcre }{ }^{-/-} \text {and } \\
\text { ADAM17-vav-cre }{ }^{-/-} \text {mice }{ }^{33,} \\
39 \text {. The latter also show } \\
\text { faster neutrophil infiltration } \\
\text { to the peritoneal cavity }{ }^{33} \text {. } \\
\text { ADAM17 is expressed in } \\
\text { human atherosclerotic } \\
\text { plaques co-localizing with } \\
\text { macrophages }{ }^{43} \text { and in } \\
\text { microparticles of human } \\
\text { atherosclerotic lesions }{ }^{44} \\
\text { ADAM17 expression is } \\
\text { associated with } \\
\text { atherosclerosis resistance } \\
\text { in LDLr-- mice }{ }^{45}\end{array}$ \\
\hline ADAM19 & TNF 37 & Not investigated & Not investigated \\
\hline ADAM28 & & $\begin{array}{l}\text { Soluble ADAM28 } \\
\uparrow a 4 \beta 1 \text {-dependent } \\
\text { cell adhesion to } \\
\text { VCAM-1 thereby } \\
\text { influencing B- } \\
\text { lymphocyte adhesion } \\
\text { /trans-migration to } \\
\text { endothelial } \\
\text { monolayers }{ }^{57}\end{array}$ & Not investigated \\
\hline ADAM33 & & $\begin{array}{l}\text { ADAM33 inhibition } \\
\uparrow \text { primary coronary } \\
\text { artery SMCs } \\
\text { migration } 46\end{array}$ & $\begin{array}{l}\text { ADAM33 is expressed in } \\
\text { human atherosclerotic } \\
\text { plaques }{ }^{46}\end{array}$ \\
\hline
\end{tabular}

"relevant to atherosclerosis 


\section{Differentiation of inflammatory cells}

When monocytes enter an atherosclerotic plaque they will differentiate into macrophages due to macrophage colony stimulating factor (M-CSF) produced in the inflamed intima ${ }^{34}$. ADAM17 can cleave M-CSF receptor ${ }^{35}$, thereby directly influencing the differentiation of macrophages. Furthermore, ADAMs could influence macrophage differentiation by targeting Notch, which has been shown to be crucial for myeloid (and lymphoid) lineage differentiation ${ }^{36}$. Besides differentiation factors, ADAM proteases have been implicated in cleavage of various cytokines and their receptors. ADAM8, 9, 10, 17 and 19 are involved in cleavage of pro-TNF producing active TNF ${ }^{37}$ and especially ADAM17 seems to play a crucial role in cleavage of TNF and TNFRs in vivo ${ }^{38}$. ADAM17-deficiency in myeloid lineages (ADAM17-LysMcre- ${ }^{-/}$) or hematopoietic cells (ADAM17-vav-cre ${ }^{-/}$) also show reduced pro-inflammatory cytokine secretion ${ }^{33,39}$. By cleavage of such cytokines and receptors, ADAMs may play a role in modulation of macrophage phenotypes and functions. TNF, for example, is a pro-inflammatory cytokine that can induce a proinflammatory M1 macrophage phenotype and is also produced by these M1 macrophages ${ }^{34,40}$. On the other hand, cleavage of the scavenger receptor CD163 by ADAM17 ${ }^{41}$ may modulate macrophage polarization and function since soluble CD163 is associated with anti-inflammatory M2 macrophage activity. Furthermore, ADAM10 and ADAM17 can cleave the IL-6 Receptor (IL-6R) in vitro and in vivo, which plays a critical role in coordination of the immune system ${ }^{42}$.

\section{In vivo evidence for a role of ADAMs in atherosclerosis}

Considering their role in neovessel formation, inflammatory cell recruitment and differentiation ADAMs most likely play an important role in atherosclerosis. Indeed, several ADAM proteases such as ADAM9, 15 and 17 have been found to be expressed in human atherosclerotic lesions, colocalizing with macrophages ${ }^{43}$. Furthermore, microparticles released from human atherosclerotic plaques were shown to contain active ADAM17, which increased TNF- $\alpha$ cleavage ${ }^{44}$. In mice, quantitative trait locus analysis has shown an association of ADAM17 expression with atherosclerosis resistance ${ }^{45}$. Others have shown that ADAM33 is also expressed in human atherosclerotic plaques and polymorphisms in ADAM8 and -33 genes are associated with atherosclerosis ${ }^{46,47}$. Furthermore, we showed that ADAM10 is expressed in macrophages/foam cells in human atherosclerotic lesions as well as ECs of plaque microvessels, linking ADAM10 to both inflammation and plaque angiogenesis ${ }^{15}$. Interestingly ADAM10 expression was 
significantly increased during plaque progression from early and advanced to ruptured atherosclerotic plaques ${ }^{15}$. The above described studies are mainly descriptive and show an association of ADAM expression with atherosclerosis. So far, only one study of a causal relation between ADAMs activity and atherosclerosis has been published. Overexpression of ADAM15, but not an inactive ADAM15 mutant, reduced atherosclerosis development in rabbits indicating an atheroprotective role of ADAM15 ${ }^{48}$. Since complete knockout mice of ADAM10 and 17 are lethal and many pharmacological inhibitors currently available are not very specific, studies using conditional knockout mice are required to determine the role of other ADAMs in this disease and to fully explore the therapeutic potential of targeting these enzymes in atherosclerotic disease. 


\section{Clinical implications}

Various ADAMs are differentially expressed in diseases, such as cancer and atherosclerosis ${ }^{49}$. Therefore, these proteases and their inhibitors may be used as biomarkers for early diagnostics and in certain cases also as prognostic markers to enable selection of the appropriate therapy ${ }^{49}$. Indeed, in cancer several ADAMs are already emerging as potential biomarkers for aiding diagnosis and predicting patient outcome ${ }^{50}$. Furthermore, a number of selective ADAM inhibitors, especially against ADAM10 and ADAM17, have been shown to have anti-cancer effects and are now undergoing clinical trials ${ }^{50}$. In line with this, ADAMs may also serve directly as biomarkers or targets for intervention in the cardiovascular field. Moreover, identification of novel molecules shed by ADAM-mediated protease (e.g. by proteomic analyses) may provide not only novel mechanistic insights of how ADAMs may modulate atherosclerotic plaque development, but could also provide novel diagnostic tools or more specific targets for intervention in cardiovascular diseases. 


\section{Conclusions}

In this review, various ADAM substrates that are involved in angiogenesis and inflammation in relation to atherosclerosis have been discussed. The list of identified substrates for ADAM proteases is already long and still growing, although future research should aim to confirm the physiological relevance of these ADAM substrates in vivo. Furthermore, there is still need for further elucidation of the mechanisms regulating ADAMs activity, substrate specificity, redundancy and distinct functions of ADAM proteases. Shedding of cell surface molecules is a complex process leading to a great variety of (patho)physiological effects essential in vascular biology and atherosclerosis (Fig. 2), i.e. either activation or inactivation of the membrane protein as well as agonistic or antagonistic effects of the released ectodomains. These soluble ectodomain could also serve as biomarkers for atherosclerosis.

Studies so far clearly indicate a crucial role for ADAM10 in endothelial cell functions, mainly mediating angiogenesis and leukocyte adhesion to the endothelium. In leukocytes, ADAM17 seems to be the predominant enzyme regulating inflammatory functions. Considering the large list of substrates and complexity of ADAMs-mediated shedding effects, ADAMs can be implicated in both pro-inflammatory and anti-inflammatory functions and in vivo studies are crucial to identify the in vivo outcome and roles of distinct ADAM proteases in cardiovascular diseases. With the introduction of (celltype specific) conditional knockout mice such in vivo research is now feasible. Elucidation of the distinct roles and mechanisms of specific ADAMs will open perspectives for the development of novel, more specific therapeutic interventions in cardiovascular diseases. 


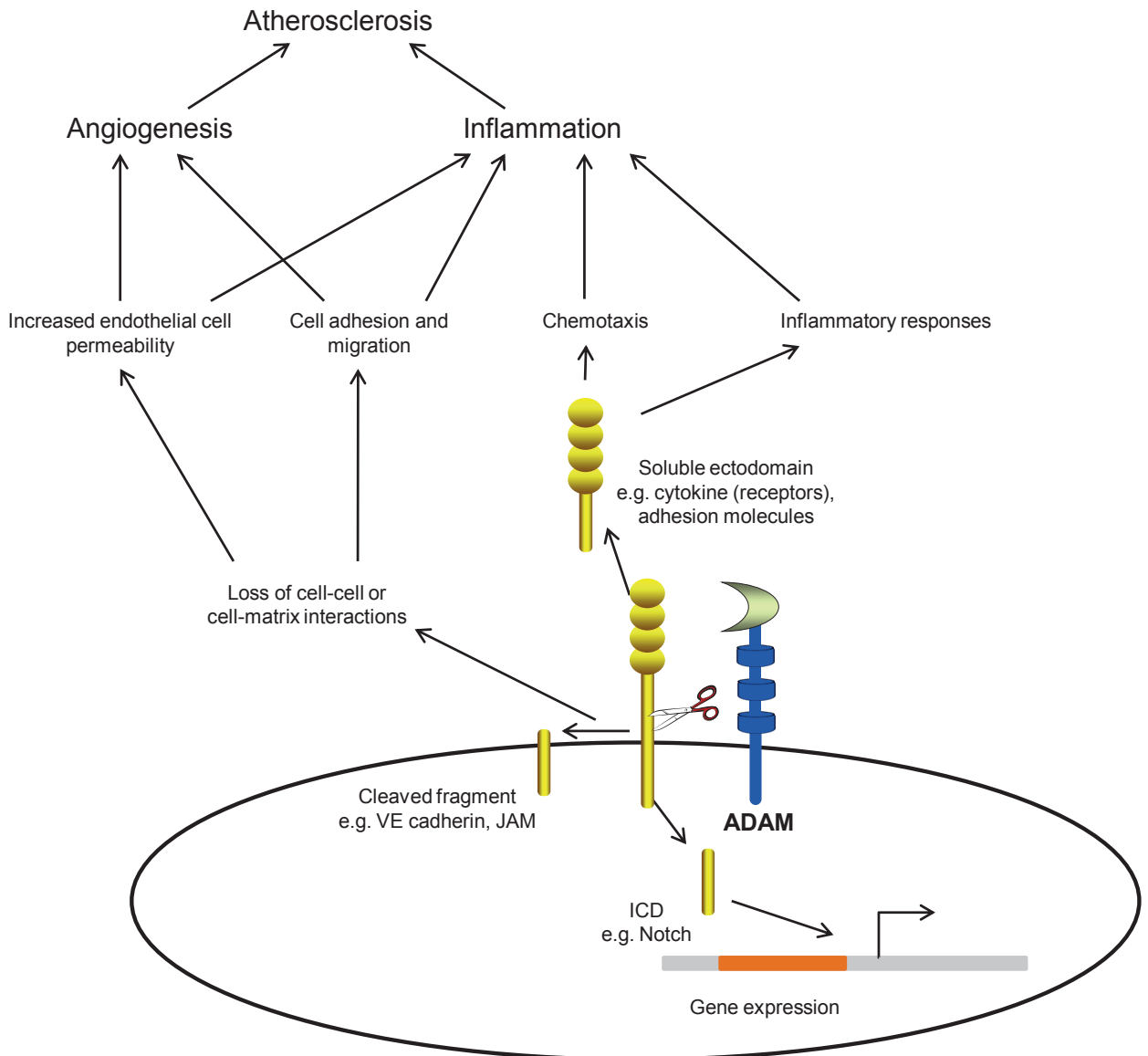

Figure 2. Schematic overview of ADAM protease functions in inflammation, angiogenesis and atherosclerosis. ICD: intracellular domain; VE-cadherin: vascular endothelial cadherin; JAM-A: Junctional Adhesion Molecule-A. 


\section{Acknowledgements}

Dr. Donners and Dr. de Winther are supported by the Netherlands Heart Foundation (Dr. E. Dekker post-doctoral fellow grant 2007T034 to Dr. Donners; Dr. E. Dekker Established Investigator grant 2007T067 to Dr. de Winther). 


\section{References}

1. Reiss K, Saftig P. The "a disintegrin and metalloprotease" (adam) family of sheddases: Physiological and cellular functions. Semin Cell Dev Biol. 2009;20:126-137

2. Edwards DR, Handsley MM, Pennington CJ. The adam metalloproteinases. Mol Aspects Med. 2008;29:258-289

3. Duffy MJ, McKiernan E, O'Donovan N, McGowan PM. The role of adams in disease pathophysiology. Clin Chim Acta. 2009;403:31-36

4. Reiss K, LudwigA, Saftig P. Breaking up the tie: Disintegrin-like metalloproteinases as regulators of cell migration in inflammation and invasion. Pharmacol Ther. 2006;111:985-1006

5. Le Gall SM, Bobe P, Reiss K, Horiuchi K, Niu XD, Lundell D, et al. Adams 10 and 17 represent differentially regulated components of a general shedding machinery for membrane proteins such as transforming growth factor alpha, I-selectin, and tumor necrosis factor alpha. Mol Biol Cell. 2009;20:1785-1794

6. Sahin U, Weskamp G, Kelly K, Zhou HM, Higashiyama S, Peschon J, et al. Distinct roles for adam10 and adam17 in ectodomain shedding of six egfr ligands. $J$ Cell Biol. 2004;164:769-779

7. Barlic J, Zhu W, Murphy PM. Atherogenic lipids induce high-density lipoprotein uptake and cholesterol efflux in human macrophages by up-regulating transmembrane chemokine cxcl16 without engaging cxcl16-dependent cell adhesion. J Immunol. 2009;182:7928-7936

8. Murphy G. Regulation of the proteolytic disintegrin metalloproteinases, the 'sheddases'. Semin Cell Dev Biol. 2009;20:138-145

9. Tellier E, Canault M, Rebsomen L, Bonardo B, Juhan-Vague I, Nalbone G, et al. The shedding activity of adam17 is sequestered in lipid rafts. Exp Cell Res. 2006;312:3969-3980

10. Lemaire-Ewing S, Lagrost L, Neel D. Lipid rafts: A signalling platform linking lipoprotein metabolism to atherogenesis. Atherosclerosis. 2012;221:303-310

11. Chen JX, Chen Y, DeBusk L, Lin W, Lin PC. Dual functional roles of tie-2/ angiopoietin in tnf-alpha-mediated angiogenesis. American journal of physiology. Heart and circulatory physiology. 2004;287:H187-195

12. Tang J, Zarbock A, Gomez I, Wilson CL, Lefort CT, Stadtmann A, et al. Adam17dependent shedding limits early neutrophil influx but does not alter early monocyte recruitment to inflammatory sites. Blood. 2011;118:786-794

13. Guaiquil VH, Swendeman S, Zhou W, Guaiquil P, Weskamp G, Bartsch JW, et al. Adam8 is a negative regulator of retinal neovascularization and of the growth 
of heterotopically injected tumor cells in mice. J Mol Med (Berl). 2010;88:497505

14. Guaiquil V, Swendeman S, Yoshida T, Chavala S, Campochiaro PA, Blobel CP. Adam9 is involved in pathological retinal neovascularization. Mol Cell Biol. 2009;29:2694-2703

15. Donners MM, Wolfs IM, Olieslagers S, Mohammadi-Motahhari Z, Tchaikovski V, Heeneman $S$, et al. A disintegrin and metalloprotease 10 is a novel mediator of vascular endothelial growth factor-induced endothelial cell function in angiogenesis and is associated with atherosclerosis. Arterioscler Thromb Vasc Biol. 2010;30:2188-2195

16. Swendeman S, Mendelson K, Weskamp G, Horiuchi K, Deutsch U, Scherle P, et al. Vegf-a stimulates adam17-dependent shedding of vegfr2 and crosstalk between vegfr2 and erk signaling. Circ Res. 2008;103:916-918

17. Koenen RR, Pruessmeyer J, Soehnlein O, Fraemohs L, Zernecke A, Schwarz N, et al. Regulated release and functional modulation of junctional adhesion molecule a by disintegrin metalloproteinases. Blood. 2009;113:4799-4809

18. Dyczynska E, Sun D, Yi H, Sehara-Fujisawa A, Blobel CP, Zolkiewska A. Proteolytic processing of delta-like 1 by adam proteases. The Journal of biological chemistry. 2007;282:436-444

19. Hartmann D, de Strooper B, Serneels L, Craessaerts K, Herreman A, Annaert W, et al. The disintegrin/metalloprotease adam 10 is essential for notch signalling but not for alpha-secretase activity in fibroblasts. Hum Mol Genet. 2002;11:26152624

20. Glomski K, Monette S, Manova K, De Strooper B, Saftig P, Blobel CP. Deletion of adam10 in endothelial cells leads to defects in organ-specific vascular structures. Blood. 2011;118:1163-1174

21. Charrier-Hisamuddin L, Laboisse CL, Merlin D. Adam-15: A metalloprotease that mediates inflammation. FASEB J. 2008;22:641-653

22. Horiuchi K, Weskamp G, Lum L, Hammes HP, Cai H, Brodie TA, et al. Potential role for adam15 in pathological neovascularization in mice. Mol Cell Biol. 2003;23:5614-5624

23. Gooz P, Gooz M, Baldys A, Hoffman S. Adam-17 regulates endothelial cell morphology, proliferation, and in vitro angiogenesis. Biochem Biophys Res Commun. 2009;380:33-38

24. Weskamp G, Mendelson K, Swendeman S, Le Gall S, Ma Y, Lyman S, et al. Pathological neovascularization is reduced by inactivation of adam17 in endothelial cells but not in pericytes. Circ Res. 2010;106:932-940

25. Phng LK, Gerhardt H. Angiogenesis: A team effort coordinated by notch. Dev Cell. 2009;16:196-208 
26. Kwak HI, Mendoza EA, Bayless KJ. Adam17 co-purifies with timp-3 and modulates endothelial invasion responses in three-dimensional collagen matrices. Matrix Biol. 2009;28:470-479

27. Virmani R, Kolodgie FD, BurkeAP, FinnAV, Gold HK, TulenkoTN, etal. Atherosclerotic plaque progression and vulnerability to rupture: Angiogenesis as a source of intraplaque hemorrhage. Arterioscler Thromb Vasc Biol. 2005;25:2054-2061

28. Ludwig A, Weber C. Transmembrane chemokines: Versatile 'special agents' in vascular inflammation. Thrombosis and haemostasis. 2007;97:694-703

29. Gough PJ, Garton KJ, Wille PT, Rychlewski M, Dempsey PJ, Raines EW. A disintegrin and metalloproteinase 10-mediated cleavage and shedding regulates the cell surface expression of cxc chemokine ligand 16. J Immunol. 2004;172:3678-3685

30. Garton KJ, Gough PJ, Philalay J, Wille PT, Blobel CP, Whitehead RH, et al. Stimulated shedding of vascular cell adhesion molecule 1 (vcam-1) is mediated by tumor necrosis factor-alpha-converting enzyme (adam 17). J Biol Chem. 2003;278:37459-37464

31. Tsakadze NL, Sithu SD, Sen U, English WR, Murphy G, D'Souza SE. Tumor necrosis factor-alpha-converting enzyme (tace/adam-17) mediates the ectodomain cleavage of intercellular adhesion molecule-1 (icam-1). J Biol Chem. 2006;281:3157-3164

32. Ballantyne CM, Entman ML. Soluble adhesion molecules and the search for biomarkers for atherosclerosis. Circulation. 2002;106:766-767

33. Long $\mathrm{C}$, Wang $\mathrm{Y}$, Herrera $\mathrm{AH}$, Horiuchi $\mathrm{K}$, Walcheck $\mathrm{B}$. In vivo role of leukocyte adam17 in the inflammatory and host responses during e. Coli-mediated peritonitis. Journal of leukocyte biology. 2010;87:1097-1101

34. Wolfs IM, Donners MM, de Winther MP. Differentiation factors and cytokines in the atherosclerotic plaque micro-environment as a trigger for macrophage polarisation. Thrombosis and haemostasis. 2011;106

35. Hiasa M, Abe M, Nakano A, Oda A, Amou H, Kido S, et al. Gm-csf and il-4 induce dendritic cell differentiation and disrupt osteoclastogenesis through m-csf receptor shedding by up-regulation of tnf-alpha converting enzyme (tace). Blood. 2009;114:4517-4526

36. Ohishi K, Katayama N, Shiku H, Varnum-Finney B, Bernstein ID. Notch signalling in hematopoiesis. Seminars in cell \& developmental biology. 2003;14:143-150

37. Bell JH, Herrera AH, Li Y, Walcheck B. Role of adam17 in the ectodomain shedding of tnf-alpha and its receptors by neutrophils and macrophages. J Leukoc Biol. 2007;82:173-176

38. Canault M, Peiretti F, Kopp F, Bonardo B, Bonzi MF, Coudeyre JC, et al. The tnf alpha converting enzyme (tace/adam17) is expressed in the atherosclerotic lesions of apolipoprotein e-deficient mice: Possible contribution to elevated 
plasma levels of soluble tnf alpha receptors. Atherosclerosis. 2006;187:82-91

39. Horiuchi K, Kimura T, Miyamoto T, Takaishi H, Okada Y, Toyama Y, et al. Cutting edge: Tnf-alpha-converting enzyme (tace/adam17) inactivation in mouse myeloid cells prevents lethality from endotoxin shock. J Immunol. 2007;179:2686-2689

40. Stoger JL, Goossens P, de Winther MP. Macrophage heterogeneity: Relevance and functional implications in atherosclerosis. Curr Vasc Pharmacol. 2010;8:233248

41. Etzerodt A, Maniecki MB, Moller K, Moller HJ, Moestrup SK. Tumor necrosis factor alpha-converting enzyme (tace/adam17) mediates ectodomain shedding of the scavenger receptor cd163. Journal of leukocyte biology. 2010;88:1201-1205

42. Garbers C, Janner N, Chalaris A, Moss ML, Floss DM, Meyer D, et al. Species specificity of adam 10 and adam 17 proteins in interleukin-6 (il-6) trans-signaling and novel role of adam10 in inducible il-6 receptor shedding. J Biol Chem. 2011;286:14804-14811

43. Oksala N, Levula M, Airla N, Pelto-Huikko M, Ortiz RM, Jarvinen O, et al. Adam-9, adam-15, and adam-17 are upregulated in macrophages in advanced human atherosclerotic plaques in aorta and carotid and femoral arteries--tampere vascular study. Ann Med. 2009;41:279-290

44. Canault M, Leroyer AS, Peiretti F, Leseche G, Tedgui A, Bonardo B, et al. Microparticles of human atherosclerotic plaques enhance the shedding of the tumor necrosis factor-alpha converting enzyme/adam17 substrates, tumor necrosis factor and tumor necrosis factor receptor-1. Am J Pathol. 2007;171:1713-1723

45. Holdt LM, Thiery J, Breslow JL, Teupser D. Increased adam17 mrna expression and activity is associated with atherosclerosis resistance in Idl-receptor deficient mice. Arteriosclerosis, thrombosis, and vascular biology. 2008;28:10971103

46. Holloway JW, Laxton RC, Rose-Zerilli MJ, Holloway JA, Andrews AL, Riaz Z, et al. Adam33 expression in atherosclerotic lesions and relationship of adam33 gene variation with atherosclerosis. Atherosclerosis. 2010;211:224-230

47. Levula M, Airla N, Oksala N, Hernesniemi JA, Pelto-Huikko M, Salenius JP, et al. Adam8 and its single nucleotide polymorphism $2662 \mathrm{t} / \mathrm{g}$ are associated with advanced atherosclerosis and fatal myocardial infarction: Tampere vascular study. Ann Med. 2009;41:497-507

48. Bultmann A, Li Z, Wagner S, Gawaz M, Ungerer M, Langer H, et al. Loss of protease activity of adam15 abolishes protective effects on plaque progression in atherosclerosis. Int J Cardiol.152:382-385

49. Turk B. Targeting proteases: Successes, failures and future prospects. Nat Rev Drug Discov. 2006;5:785-799 
50. Duffy MJ, Mullooly M, O'Donovan N, Sukor S, Crown J, Pierce A, et al. The adams family of proteases: New biomarkers and therapeutic targets for cancer? Clin Proteomics. 2011;8:9

51. Huovila AP, Turner AJ, Pelto-Huikko M, Karkkainen I, Ortiz RM. Shedding light on adam metalloproteinases. Trends Biochem Sci. 2005;30:413-422

52. Murphy G. The adams: Signalling scissors in the tumour microenvironment. Nat Rev Cancer. 2008;8:929-941

53. Nagano O, Murakami D, Hartmann D, De Strooper B, Saftig P, Iwatsubo T, et al. Cell-matrix interaction via cd44 is independently regulated by different metalloproteinases activated in response to extracellular ca(2+) influx and pkc activation. J Cell Biol. 2004;165:893-902

54. Hundhausen C, Schulte A, Schulz B, Andrzejewski MG, Schwarz N, von Hundelshausen $P$, et al. Regulated shedding of transmembrane chemokines by the disintegrin and metalloproteinase 10 facilitates detachment of adherent leukocytes. J Immunol. 2007;178:8064-8072

55. Schwarz N, Pruessmeyer J, Hess FM, Dreymueller D, Pantaler E, Koelsch A, et al. Requirements for leukocyte transmigration via the transmembrane chemokine cx3cl1. Cell Mol Life Sci. 2010;67:4233-4248

56. Garton KJ, Gough PJ, Raines EW. Emerging roles for ectodomain shedding in the regulation of inflammatory responses. Journal of leukocyte biology. 2006;79:1105-1116

57. McGinn OJ, English WR, Roberts S, Ager A, Newham P, Murphy G. Modulation of integrin alpha4beta1 by adam28 promotes lymphocyte adhesion and transendothelial migration. Cell Biol Int. 2011;35:1043-1053 


\section{Chapter 3}

\section{Hematopoietic ADAM8 deficiency does not influence atherosclerotic plaque area or composition, despite ADAM8 upregulation during plaque progression}

Emiel P.C. van der Vorst, Kosta Theodorou, Thomas Theelen, Bart Smeets, Ine M.J. Wolfs, Mike Jeurissen, Marion J. Gijbels, Erwin Wijnands, Andreas Ludwig, Jörg W. Bartsch, Erik A.L. Biessen, Marjo M.P.C. Donners

In preparation 


\section{Abstract}

Atherosclerosis is characterized by the accumulation of lipids and inflammatory cells in the vessel wall, which eventually can lead to major clinical complications like myocardial infarction or stroke. Several cytokines, chemokines and adhesion molecules were seen to play a crucial role in its pathogenesis. A Disintegrin And Metalloproteases (ADAMs) are a family of proteases capable of shedding many of these immune-modulators and could thereby contribute to atherosclerosis development. Although carriers of a polymorphism in the ADAM8 gene have a significantly greater risk of myocardial infarction, associating ADAM8 to atherosclerosis, a causal role of ADAM8 in atherosclerosis has not been demonstrated so far. In this study, we show increased ADAM8 expression, especially in foamy macrophages, upon progression of human lesions. Surprisingly, however, $\mathrm{LDLr}^{-1-}$ mice with hematopoietic ADAM8 deficiency showed unchanged lesion size or composition, even though several circulating leukocyte subsets including granulocytes and monocytes, were significantly reduced in these mice. Further investigation using total body ADAM8 deficient mice is warranted to fully elucidate a causal role of ADAM8 in atherosclerosis. 


\section{Introduction}

The primary antecedent of cardiovascular disease (CVD), the main cause of death worldwide, is atherosclerosis ${ }^{1}$. Atherosclerosis is a chronic inflammatory disease, characterized by the accumulation of lipids and inflammatory cells in the vessel wall. Atherosclerotic lesions can eventually narrow or rupture, leading to thrombus formation, thereby occluding the artery ${ }^{2,3}$.

A family of proteases that has widely been associated with atherosclerosis and vascular disease are the A Disintegrin And Metalloproteases (ADAMs) 4, 5. Many ADAMs are expressed on cell-types important in atherosclerosis development, like leukocytes, endothelial cells and smooth muscle cells ${ }^{4,5}$. The best known members of this family are ADAM10 and ADAM17, whose expression has been shown to be associated with plaque progression ${ }^{6}$. These proteases have also been shown to be important in many relevant processes for atherosclerosis development, like endothelial permeability, leukocyte adhesion and transmigration ${ }^{4,5}$.

Besides these two proteases, also ADAM8 has recently been associated with atherosclerosis, since recent studies by Raitoharju et al. revealed that carriers of a specific ADAM8 polymorphism (Rs2275725) display an increased risk of myocardial infarction, in two independent cohorts ${ }^{7}$. ADAM8 is expressed on several cell-types, including bone marrow-derived and peripheral blood derived lymphoid and myeloid cells ${ }^{8}$. ADAM8 is associated with many pathological processes, mostly inflammatory diseases. For example, ADAM8 expression correlated with the degree of joint inflammation in patients with rheumatoid arthritis ${ }^{9}$. One of the known targets of ADAM8 is for example CD62L, a mediator of leukocyte infiltration into the vessel wall, which is a key step in lesion development ${ }^{9}$. More strikingly, it has been shown that ADAM8 expression increases upon atherosclerotic lesion development ${ }^{10,11 .}$

However, until now only association studies have been reported regarding ADAM8 in atherosclerosis development. In this study we investigated the hypothesis that hematopoietic ADAM8 has a causal effect in the development and progression of atherosclerotic lesions. Therefore atherosclerosis prone $\mathrm{LDLr}^{-/}$mice were lethally irradiated and reconstituted with either ADAM8 deficient bone marrow or bone marrow from littermate controls. 
Although we could confirm the association of ADAM8 expression and lesion development in humans, we could not observe any difference in plaque size or composition comparing hematopoietic ADAM8 deficient and wildtype mice. 


\section{Methods}

\section{Immunohistochemistry of human tissues}

Human carotid artery plaque tissue was obtained by endarterectomy, for more detail see ${ }^{12}$. Lesions were fixed in paraformaldehyde and paraffin embedded. Sections were incubated with primary antibody (1:25 diluted, AF1031, R\&D systems) against human ADAM8, followed by detection with a HRP-labelled rabbit anti-goat antibody (1:400 diluted, E0466, Dako) and staining with the ABC kit (Vector Labs, USA).

\section{PCR analysis of human tissues}

Human carotid atherosclerotic lesions were obtained from surgical interventions on human subjects that aimed to remove stenosis in the common carotid artery. Segments were snap-frozen and subsequently RNA was isolated using the guanidine isothiocyanate/CsCl method ${ }^{13}$. RNA was further purified and concentrated using RNeasy mini columns (Qiagen, Germany). Total RNA was normalized and reverse transcribed using iScript (BioRad). Quantitative PCR was performed using $10 \mathrm{ng}$ cDNA, $300 \mathrm{nM}$ of each primer, and SensiMix (Quantace-Bioline). All gene expression levels were corrected for cyclophilin A and $\beta$-actin as housekeeping genes.

\section{Bone marrow derived macrophage isolation and culture}

Bone marrow cells were isolated from femurs and tibiae of either ADAM8 deficient (ADAM8 ${ }^{-/}$) or wildtype (wt) mice. Cells were cultured in RPMI-1640 (GIBCO Invitrogen, Breda, the Netherlands) with 10\% heat-inactivated fetal calf serum (Bodinco B.V. Alkmaar, the Netherlands), penicillin $(100 \mathrm{U} / \mathrm{ml})$, streptomycin $(100 \mu \mathrm{g} / \mathrm{ml})$ and L-glutamine $2 \mathrm{mM}$ (all GIBCO Invitrogen, Breda, the Netherlands) supplemented with 15\% L929-conditioned medium (LCM) for 8-9 days to generate bone marrow-derived macrophages (BMDMs), as described previously ${ }^{14}$.

Macrophages were seeded at 350,000 cells per well in 24 wells plates and incubated 6-24 hrs with 0-10 ng/ml LPS. IL-12, IL-10 and TNF- $\alpha$ ELISA assays (all Invitrogen) were performed on conditioned medium according to manufacturer's instructions. Analysis was performed using a micro-plate reader (Bio-Rad) at $450 \mathrm{~nm}$. NO production was measured in conditioned medium using Griess reagent $\left(2.5 \% \quad \mathrm{H}_{3} \mathrm{PO}_{4}, 1 \%\right.$ sulfanilamide, $0.1 \%$ naphthalene diamine dihydrochloride). Analysis was performed using a micro-plate reader (Bio-Rad) at $550 \mathrm{~nm}$. 
All animal experiments were approved by the Animal Ethics Committee of the Maastricht University (permit number 2012-065).

\section{Bone marrow transplantation}

Ten to twelve week old female ADAM8 ${ }^{-/}$or wt mice were used as donor mice ( $\mathrm{n}=2$ per group). Forty female $L D L r^{\prime-}$ recipient mice backcrossed onto a C57BI6 background for more than 10 generations were obtained from inhouse breeding. Bone marrow transplantations were performed as described elsewhere ${ }^{15}$. In short, LDLr-1- mice were lethally irradiated with 6 Gy the day before and the day of transplantation. Mice were reconstituted with $1 \times 10^{5}$ bone marrow cells isolated from wildtype or ADAM8 ${ }^{-/-}$mice. After recovery for five weeks after transplantation mice were given a Western-type diet (WTD), containing $16 \%$ fat and $0.15 \%$ cholesterol (Hope Farms, The Netherlands). Before western type diet feeding, mice were fasted for $4 \mathrm{hrs}$, after which blood samples were drawn from the tail vein for analysis of plasma lipids and chimerism. Additional blood analysis was performed at 5 and 10 weeks of WTD. The chimerism in transplanted mice was determined on DNA from blood leukocytes as described previously ${ }^{14}$.

\section{Morphometry and immunohistochemistry of murine tissues}

After 10 weeks of WTD feeding, mice were anesthetized and euthanized. Mouse hearts were dissected and snap frozen in optimum cutting temperature (OCT). Atherosclerosis development (lesion size and progression stage) was determined as previously described ${ }^{16}$, with a slight modification in plaque classification. Plaques were classified as early (foam cell rich, but lacking a necrotic core), moderately advanced (containing a fibrotic cap and often a necrotic core, but no medial macrophage infiltration) and advanced lesions, typified by medial macrophage infiltrates, elastic lamina degradation and more pronounced necrosis and fibrosis. Serial sections $(7 \mu \mathrm{m})$ of the aortic root were cut and stained with toluidine blue for morphometric analysis and routine qualitative examination of collagen content, necrosis, foam cell content and amount of inflammatory cells (using scores from 0 (absent) to 3 (high abundance)). Moma2 (an antibody recognizing monocytes/macrophages; gift from G. Kraal), NIMP-1 (neutrophil specific antibody directed against Ly6G; gift from P. Heeringa) and Sirius Red staining was used for the detection of monocytes/macrophages, neutrophils and collagen respectively. 


\section{Plasma Lipid analysis}

Mice were fasted for $4 \mathrm{hrs}$, before blood was drawn for plasma lipid analyses. Total plasma cholesterol and triglyceride levels as well as cholesterol in lipoprotein fractions were determined using standard enzymatic kits according to manufacturer's protocols (Sigma-Aldrich, Zwijndrecht, the Netherlands).

\section{Lipid uptake by peritoneal macrophages}

Resident peritoneal macrophages were obtained by flushing the peritoneal cavity with ice-cold PBS followed by culturing in RPMI 1640 culture medium containing $10 \%$ ( $\mathrm{vol} / \mathrm{vol}) \mathrm{FCS}$, penicillin $(100 \mathrm{U} / \mathrm{ml})$, streptomycin $(100 \mathrm{ug} / \mathrm{ml})$, and L-glutamine $2 \mathrm{mM}$ (all GIBCO Invitrogen). After overnight attachment on cover slips, floating cells were removed. Attached cells were stained with Oil Red $\mathrm{O}$ and counterstained with haematoxylin.

\section{Flow cytometry analysis}

Absolute circulating leukocyte subset numbers were determined by flow cytometry calibrated using Trucount Beads (BD, New Jersey, U.S.). Leukocytes were defined as CD45+, T-cells as CD45+ CD $3^{+} \mathrm{NK} 1.1^{-}$, NK cells as $\mathrm{CD}_{45^{+}} \mathrm{CD} 3^{-} \mathrm{NK} 1.1^{+}$, B cells as $\mathrm{CD} 45^{+} \mathrm{CD} 3^{-} \mathrm{NK} 1.1^{-} \mathrm{B} 220^{+}$, granulocytes as CD45+ CD3- NK1.1- B220- CD11 b+ Ly6G $^{+}$and monocytes as CD45+ CD3NK1.1- B220- CD11 b+ Ly6G. A list of applied antibodies is given in Table 1.

Table 1. Antibodies used for flow cytometry

\begin{tabular}{|l|l|l|}
\hline \multicolumn{1}{|c|}{ Antigen } & \multicolumn{1}{c|}{ Clone } & \multicolumn{1}{c|}{ Company } \\
\hline CD16/32 & 93 & eBioscience \\
\hline CD45 & $30-F 11$ & Biolegend \\
\hline CD3e & 145-2c11 & eBioscience \\
\hline NK1.1 & PK136 & BD \\
\hline Ly6G & 1A8 & BD \\
\hline CD11b & M1/70 & BD \\
\hline B220 & RA3-6B2 & BD \\
\hline
\end{tabular}




\section{Statistical analysis}

Data are presented as mean \pm the standard error of the mean (SEM). All statistical analyses were performed using the Prism program (GraphPad Software Inc, San Diego, U.S.). Differences between treatment groups were evaluated for statistical significance with two-tailed, unpaired Student's t-test. Significance was accepted at the level of $p<0.05$. ${ }^{*} p<0.05$; ${ }^{* *} p<0.01$; ${ }^{* * *} p<0.001$. 


\section{Results}

\section{ADAM8 is expressed in human atherosclerotic lesions and associated with plaque progression}

A previous study in which polymorphisms in ADAM8 were found to correlate with the incidence of acute myocardial infarction, a phenomenon which is mainly caused by atherosclerotic plaque rupture, prompted us to investigate ADAM8 gene expression in different human atherosclerotic plaques ${ }^{7}$. Tissue lysates from early, stable and unstable human lesions showed increasing ADAM8 mRNA expression with progression of disease (Fig. 1A). Additionally, immunohistochemical staining of ADAM8 in human carotid atherosclerotic plaques also confirmed expression of ADAM8 in lesions at the protein level. Strikingly, ADAM8 expression was most prominent in the shoulder regions of the plaque, an area composed of mainly inflammatory cells and therefore considered to be the most active inflammatory region of the plaque (Fig. 1B). Plaque macrophages located near the lumen were less positive for ADAM8, whereas foamy macrophages, laying deeper in the vessel wall, showed high expression of ADAM8 (Fig. 1C), suggesting that ADAM8 might play a more important role at later stages of lesion progression. 
A

B

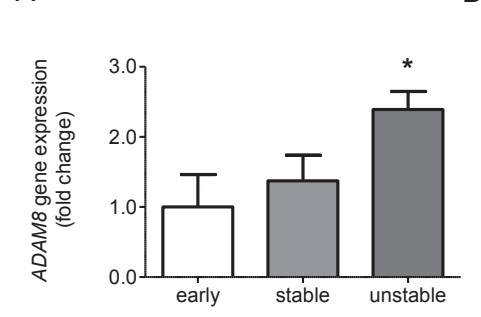

C
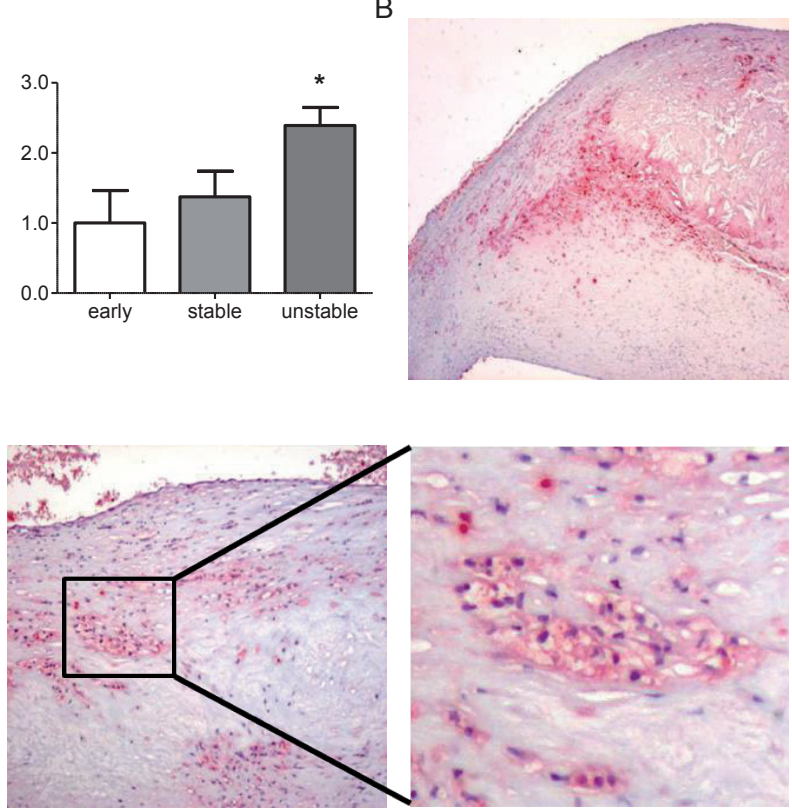

Figure 1. ADAM8 expression in human atherosclerotic plaques. Relative gene expression of ADAM8 in early, stable and unstable human plaques. Values have been corrected for GAPDH expression (A, $\mathrm{n}=5-7)$. Immunohistochemical stainings for ADAM8 of human atherosclerotic lesions (B-C). Representative image of shoulder region (B, magnification $4 \mathrm{x}$ ) and region rich of foamy macrophages (C, magnification $4 x$, insert $20 x$ ) is shown. 


\section{ADAM8 deficient macrophages show overall reduced cytokine secretion}

Macrophages are the main inflammatory cell-type in mouse atherosclerotic plaques, and are able to produce various pro- and anti-inflammatory mediators. Since ADAMs play a crucial role in cytokine shedding, we examined the role of ADAM8 in LPS-induced cytokine production by bone marrow derived macrophages (BMDMs). TNF- $\alpha$ secretion was significantly reduced in ADAM8 deficient BDMDs (Fig. 2A), while secretion of IL-12, NO and IL-10 was only slightly reduced (Fig. 2B-D).

A

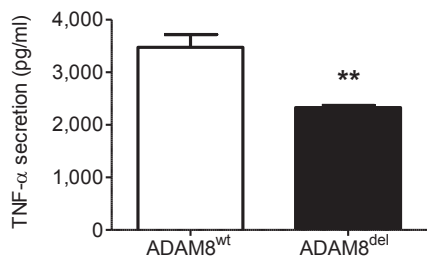

C

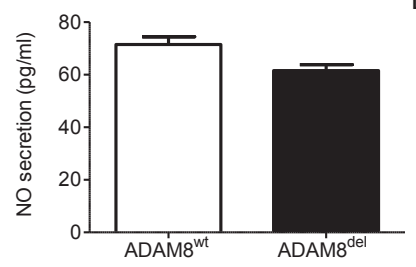

B

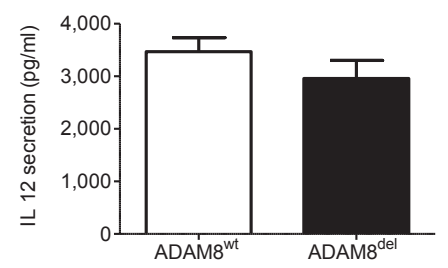

D

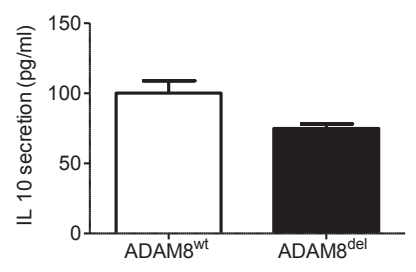

Figure 2. ADAM8 deficient BMDMs show reduced TNF- $\alpha$ secretion. Pro-inflammatory mediator (A-C) and anti-inflammatory cytokine (D) production by bone marrow-derived macrophages (BMDMs) after activation by LPS (6 hrs for TNF- $\alpha$ and IL-10; 24 hrs for IL-12 and NO); n=3/group. 


\section{Systemic reduction of inflammatory cell-types of hematopoietic ADAM8 deficiency in LDLr ${ }^{-/}$mice}

ADAM8 expression is associated with human plaque progression and instability; however, a causal role for ADAM8 in atherosclerosis development still needs to be established. Therefore, we investigated the effect of hematopoietic deficiency of ADAM8 on lesion development by reconstituting lethally irradiated $\mathrm{LDLr}^{-1-}$ mice with bone marrow from $\mathrm{ADAM}^{-1-}$ mice $\left(A D A M 8^{\mathrm{del}}\right)$ or littermate controls (ADAM8 ${ }^{\mathrm{wt}}$ ). After reconstitution, mice were fed a Western-type diet (WTD) for 10 weeks after which lesion development was assessed. Overall chimerism determination showed more than $95 \%$ repopulation for both genotypes (data not shown). No differences between groups were found in body weight $\left(24.6 \pm 0.4 \mathrm{~g}\right.$ for ADAM8 $^{\text {wt }}$ vs $24.5 \pm 0.7 \mathrm{~g}$ for ADAM8 ${ }^{\text {del }}$ mice at sacrifice) and WTD induced weight gain.

Plasma cholesterol before and after WTD feeding were not different between genotypes (Fig. 3A). Although triglyceride levels in ADAM8 ${ }^{\text {del }}$ mice were significantly increased (14\%) compared to ADAM8 ${ }^{\text {wt }}$ mice at baseline, no differences were observed upon WTD (Fig. 3B).

Furthermore, circulating leukocyte subsets were analyzed by flow cytometry in transplanted animals at baseline (0 weeks) and at sacrifice (10 weeks) (Fig. 3C-D). At baseline and after 10 weeks WTD total number of CD45 leukocytes in blood was significantly reduced in ADAM8 ${ }^{\text {del }}$ mice, compared to ADAM8 ${ }^{\text {wt }}$ mice. ADAM8 deficiency associated leukopenia affected almost all major leukocyte populations measured, but was most prominent for Ly6G ${ }^{+}$CD $11 b^{+}$granulocytes. 
A

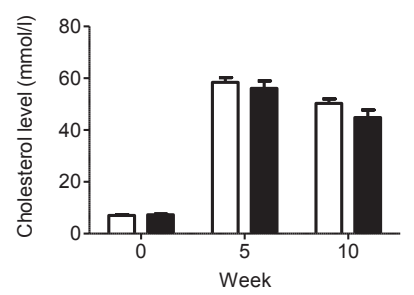

C

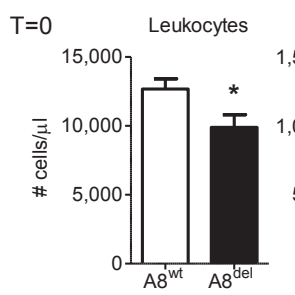

D

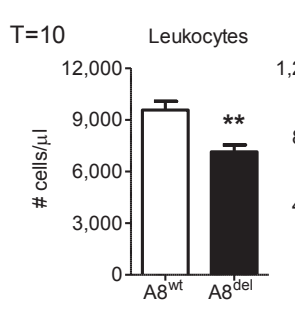

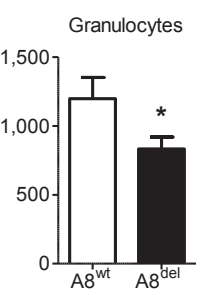
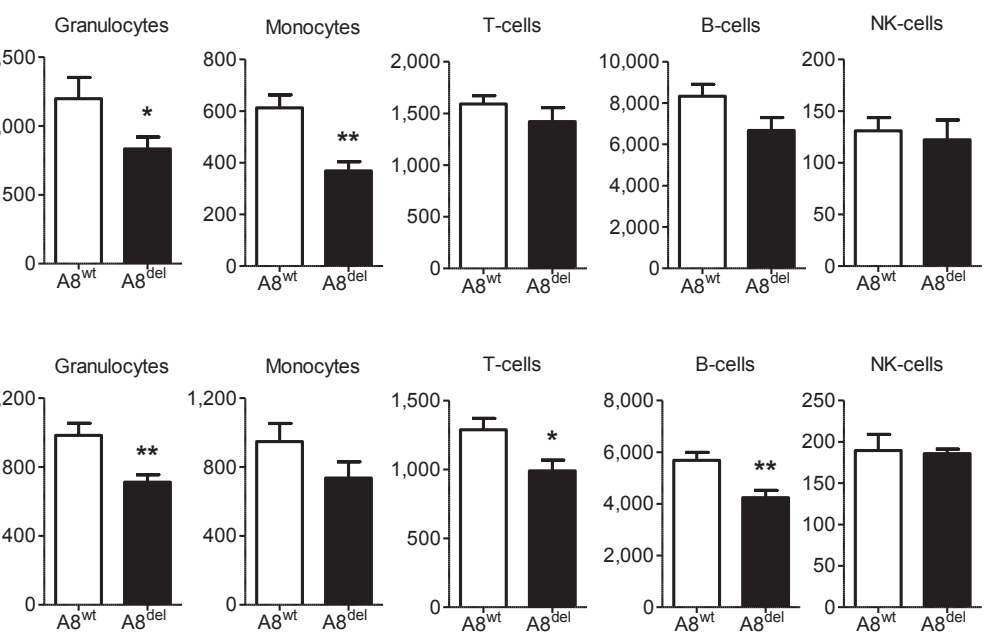

NK-cells

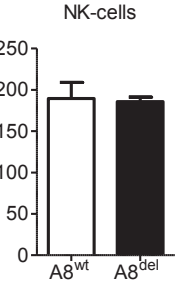

Figure 3. Reduction of circulating inflammatory cells in hematopoietic ADAM8 deficient mice. Plasma cholesterol (A) and triglyceride (B) levels of ADAM8 ${ }^{\text {wt }}$ and ADAM8 ${ }^{\text {del }}$ mice; $n=20$ / group. FACS analyses showing absolute numbers of leukocytes, granulocytes, monocytes, T-cells, B-cells and NK-cells in blood of ADAM8 ${ }^{\text {wt }}$ and ADAM8 ${ }^{\text {del }}$ mice at $t=0$ weeks $(\mathbf{C})$ or $t=10$ weeks (D) of Western-type diet feeding; $n=10$ /group. 


\section{Hematopoietic ADAM8 deficiency does not affect atherosclerotic lesion size or composition}

Atherosclerotic lesion development in the aortic root was assessed after 10 weeks of WTD feeding. Hematopoietic ADAM8 deficiency did not result in differences in lesion area (Fig. 4A-C) or progression (Fig. 4D). Further routine pathological examination for atherosclerotic lesion composition, i.e. collagen content, necrosis, foam cell content, amount of inflammatory cells, monocyte adhesion, granulocyte content and adventitial influx of leukocytes, showed no significant differences between ADAM8 ${ }^{\text {del }}$ and littermate control mice (Fig. 4E), even though the amount of circulating inflammatory cells and granulocyte numbers were slightly decreased in ADAM8 ${ }^{\text {del }}$ mice.

A

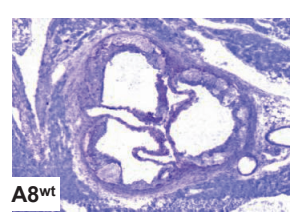

D
B

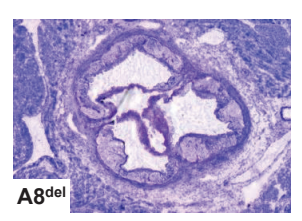

C

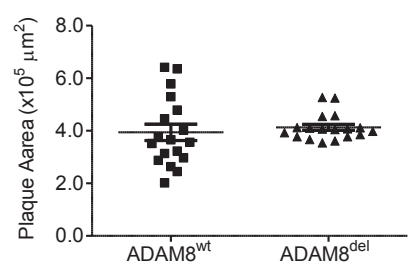

$\mathrm{E}$
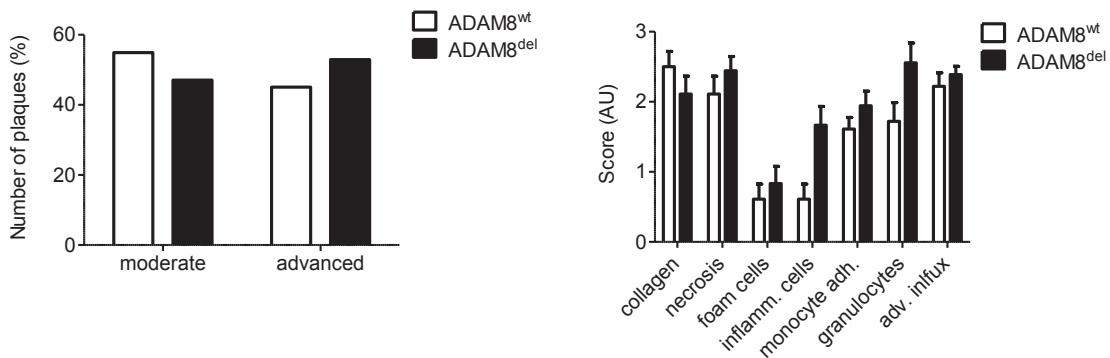

Figure 4. Hematopoietic ADAM8 deficiency does not affect plaque size. Representative pictures of toluidine blue-stained sections of the aortic root of ADAM8 ${ }^{\text {wt }}(\mathbf{A})$ and ADAM8 $8^{\text {del }}$ (B) mice, magnification 40x. Quantification of plaque area (C), plaque classification (D) and routine pathological examination of plaque composition $(E)$; $n=20 / g r o u p$. 
Subsequent quantitative analysis of plaque composition using immunohistochemistry confirmed that macrophage (Fig. 5A-D) and collagen (Fig. 5E-H) content of plaques were not influenced by ADAM8 deficiency. Granulocyte content tended to be higher in plaques of ADAM8 ${ }^{\text {del }}$ mice; and in particular in moderately advanced lesions (Fig. 5I-L).

Altogether, these data show that, despite reduced circulating leukocyte levels, hematopoietic ADAM8 deficiency does not influence atherosclerotic lesion development or composition.

A

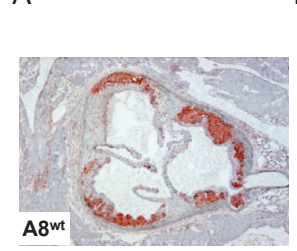

B

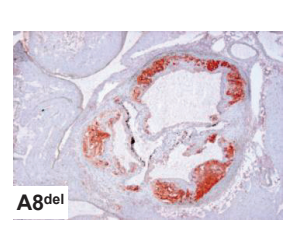

C

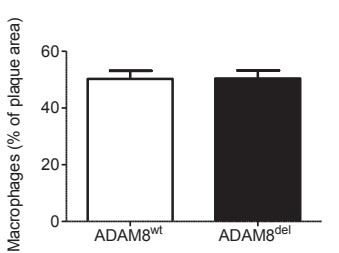

E

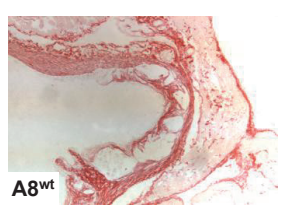

F
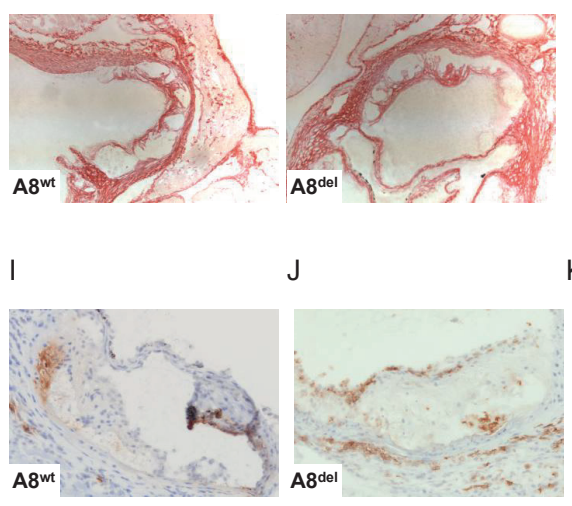

J

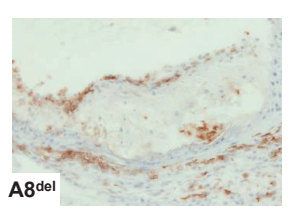

G

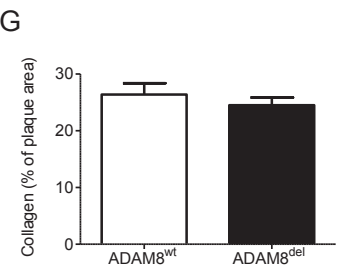

K

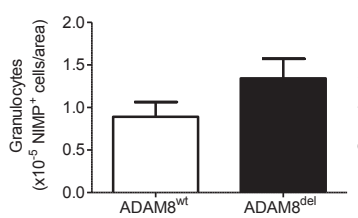

D

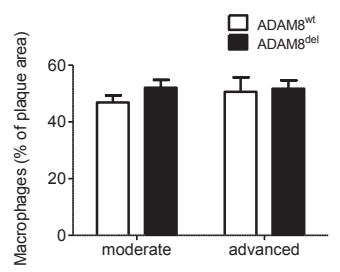

$\mathrm{H}$

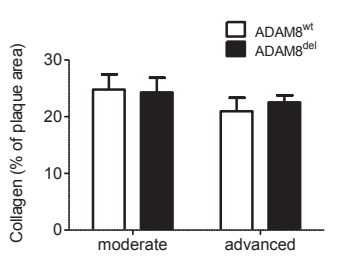

L

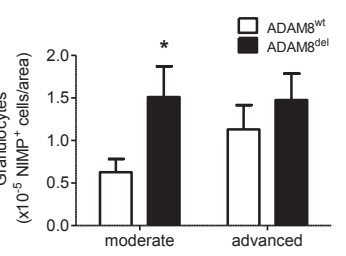

Figure 5. Atherosclerotic lesion composition is not influenced by hematopoietic ADAM8 deficiency. Representative pictures and quantification of $\mathrm{MOMA}^{+}$macrophages (A-D), Siriusred stained collagen (E-H) and $\mathrm{NIMP}^{+}$granulocytes (I-L) of $\mathrm{ADAM}^{\mathrm{wt}}$ and ADAM8 ${ }^{\text {del }}$ mice, magnification 40x; $n=17-20 /$ group. 


\section{Discussion}

In this study we investigated the role of ADAM8 in atherosclerosis development. We show that ADAM8 expression is increased in unstable human atherosclerotic lesion. Additionally, ADAM8 was shown to be highly expressed in the shoulder regions of the atherosclerotic plaques, which are the active regions of the lesions filled with (foamy) macrophages ${ }^{17}$. Specifically, these foamy macrophages were shown to have high ADAM8 expression, suggesting that ADAM8 plays an increasingly important role upon lesion progression.

Remarkably, despite these clear associations, we did not observe any effects of hematopoietic ADAM8 deficiency on atherosclerosis development in mice, apart from mild leukopenia under normo- (baseline) and hyperlipidemic conditions (10 weeks of WTD feeding), that affected almost all major leukocyte populations, such as T- and B-cells (only after WTD feeding) and granulocytes (both conditions). It has already been described that ADAM8 deficiency results in decreased circulating T-lymphocytes and an increased amount of T-lymphocytes in the thymus, probably because the ADAM8 deficient T-lymphocytes are not able to migrate out of the thymus 18. Further investigation is needed to elucidate the mechanisms behind this effect of chronic WTD feeding on the T-lymphocyte development or release from thymus, and to determine the plaque T-lymphocyte content. More importantly, we observed significant reductions in myeloid cells, and in particular in granulocytes, which are key players in the development of atherosclerosis, in ADAM8 ${ }^{\text {del }}$ mice compared to ADAM8 ${ }^{\text {wt }}$ at baseline and after 10 weeks WTD. The reduction in leukocytes observed at baseline could be the result of repopulation problems, due to the ADAM8 deficiency. A main target of ADAM8 is CD62L, which is expressed on leukocytes, in particular on granulocytes and is important for the egress from the bone marrow into the bloodstream ${ }^{9,19}$. Conceivably, ADAM8 deficient leukocytes do not shed CD62L, important for this migration and therefore fewer leukocytes can be released and/or are more slowly released, leading to decreased circulating leukocyte levels, as CD62L acts as a retention factor for granulocytes in the bone marrow ${ }^{20}$. Further research is needed to elucidate the involved mechanisms. 
Surprisingly, despite the avid expression in plaque, the reported role in a range of inflammatory processes relevant to atherosclerosis, and the leukopenia, we did not observe a causal effect of ADAM8 in atherosclerosis development and progression. Not only plaque area, but also composition was comparable between ADAM8 ${ }^{\text {del }}$ and ADAM8 ${ }^{\text {wt }}$ mice. These remarkable results might be explained by the fact that we used mice that had only a hematopoietic ADAM8 deficiency, although we observed a lot of ADAM8 expression in foam cells of human lesions. Immunohistochemical stainings should determine what the expression profile of ADAM8 in mouse lesions is, to determine the possible involvement of ADAM8 expressed on other cell-types. Especially since, in contrast to all other ADAMs, ADAM8 exists in two physiologically active forms, namely a classical membrane bound and a soluble form ${ }^{21}$. To date, a lot is still unknown regarding this soluble ADAM8, e.g. exact activity and targets, mechanism of synthesis and response to certain stimuli. Therefore in our model of hematopoietic ADAM8 deficiency, it is plausible that soluble ADAM8 derived from non-hematopoietic cells like endothelial cells can still influence the hematopoietic cells, thereby compensating for the hematopoietic deficiency. Alternatively, other ADAMs may compensate for the loss of ADAM8, which in certain conditions is already observed for ADAM 10 and $-17^{22}$, explaining the absence of atherosclerotic effects. Another possibility is that the role of ADAM8 is different for the various atherosclerotic developmental stages, as ADAM8 was much higher expressed in unstable human lesions compared to stable lesions. Further investigation of ADAM8 at different developmental stages would therefore be of great interest.

Since ADAM8 has not been investigated thoroughly yet, it is conceivable that ADAM8 has more atherosclerosis-relevant substrates than currently identified. Although ADAM8 plays important roles in physiological and developmental processes like angiogenesis and neurogenesis, total body ADAM8 deficient mice are still viable and show no pathological abnormalities 23-25. These total body ADAM8 deficient mice should be used to further investigate the causal role of ADAM8 in atherosclerosis, before making more general conclusions. This study already shows a clear association of ADAM8 and atherosclerotic lesion development in humans, although hematopoietic ADAM8 deficiency in mice did not affect plaque size or composition, possibly due to compensation by active soluble ADAM8 derived from other nonhematopoietic cells. Therefore, follow-up studies using full body ADAM8 deficient mice will unravel a potential causal role for ADAM8 in atherosclerotic lesion development. 


\section{References}

1. Hansson GK, Hermansson A. The immune system in atherosclerosis. Nature immunology. 2011;12:204-212

2. Finn AV, Nakano M, Narula J, Kolodgie FD, Virmani R. Concept of vulnerable/ unstable plaque. Arteriosclerosis, thrombosis, and vascular biology. 2010;30:12821292

3. Lusis AJ. Atherosclerosis. Nature. 2000;407:233-241

4. Dreymueller D, Pruessmeyer J, Groth E, Ludwig A. The role of adam-mediated shedding in vascular biology. European journal of cell biology. 2012;91:472-485

5. van der Vorst EP, Keijbeck AA, de Winther MP, Donners MM. A disintegrin and metalloproteases: Molecular scissors in angiogenesis, inflammation and atherosclerosis. Atherosclerosis. 2012;224:302-308

6. Donners MM, Wolfs IM, Olieslagers S, Mohammadi-Motahhari Z, Tchaikovski V, Heeneman S, et al. A disintegrin and metalloprotease 10 is a novel mediator of vascular endothelial growth factor-induced endothelial cell function in angiogenesis and is associated with atherosclerosis. Arteriosclerosis, thrombosis, and vascular biology. 2010;30:2188-2195

7. Raitoharju E, Seppala I, Levula M, Kuukasjarvi P, Laurikka J, Nikus K, et al. Common variation in the adam8 gene affects serum sadam8 concentrations and the risk of myocardial infarction in two independent cohorts. Atherosclerosis. 2011;218:127-133

8. Edwards DR, Handsley MM, Pennington CJ. The adam metalloproteinases. Molecular aspects of medicine. 2008;29:258-289

9. Gomez-Gaviro M, Dominguez-Luis M, Canchado J, Calafat J, Janssen H, Lara-Pezzi $\mathrm{E}$, et al. Expression and regulation of the metalloproteinase adam-8 during human neutrophil pathophysiological activation and its catalytic activity on I-selectin shedding. Journal of immunology. 2007;178:8053-8063

10. Levula M, Airla N, Oksala N, Hernesniemi JA, Pelto-Huikko M, Salenius JP, et al. Adam8 and its single nucleotide polymorphism $2662 \mathrm{t} / \mathrm{g}$ are associated with advanced atherosclerosis and fatal myocardial infarction: Tampere vascular study. Annals of medicine. 2009;41:497-507

11. Pelisek J, Pongratz J, Deutsch L, Reeps C, Stadlbauer T, Eckstein HH. Expression and cellular localization of metalloproteases adams in high graded carotid artery lesions. Scandinavian journal of clinical and laboratory investigation. 2012;72:648-656

12. Goossens P, Gijbels MJ, Zernecke A, Eijgelaar W, Vergouwe MN, van der Made I, et al. Myeloid type i interferon signaling promotes atherosclerosis by stimulating 
macrophage recruitment to lesions. Cell metabolism. 2010;12:142-153

13. Chomczynski P, Sacchi N. Single-step method of rna isolation by acid guanidinium thiocyanate-phenol-chloroform extraction. Analytical biochemistry. 1987;162:156-159

14. Kanters E, Pasparakis M, Gijbels MJ, Vergouwe MN, Partouns-Hendriks I, Fijneman $\mathrm{RJ}$, et al. Inhibition of nf-kappab activation in macrophages increases atherosclerosis in Idl receptor-deficient mice. The Journal of clinical investigation. 2003;112:1176-1185

15. Donners MM, Wolfs IM, Stoger LJ, van der Vorst EP, Pottgens CC, Heymans S, et al. Hematopoietic mir155 deficiency enhances atherosclerosis and decreases plaque stability in hyperlipidemic mice. PloS one. 2012;7:e35877

16. Gijbels MJ, van der Cammen M, van der Laan LJ, Emeis JJ, Havekes LM, Hofker $\mathrm{MH}$, et al. Progression and regression of atherosclerosis in apoe3-leiden transgenic mice: An immunohistochemical study. Atherosclerosis. 1999;143:1525

17. Moore KJ, Tabas I. Macrophages in the pathogenesis of atherosclerosis. Cell. 2011;145:341-355

18. Gossens K, Naus S, Hollander GA, Ziltener HJ. Deficiency of the metalloproteinasedisintegrin adam8 is associated with thymic hyper-cellularity. PloS one. 2010;5:e12766

19. van Eeden SF, Miyagashima R, Haley L, Hogg JC. A possible role for I-selectin in the release of polymorphonuclear leukocytes from bone marrow. The American journal of physiology. 1997;272:H1717-1724

20. Kassirer M, Zeltser D, Gluzman B, Leibovitz E, Goldberg Y, Roth A, et al. The appearance of I-selectin(low) polymorphonuclear leukocytes in the circulating pool of peripheral blood during myocardial infarction correlates with neutrophilia and with the size of the infarct. Clinical cardiology. 1999;22:721-726

21. Higuchi Y, Yasui A, Matsuura K, Yamamoto S. Cd156 transgenic mice. Different responses between inflammatory types. Pathobiology : journal of immunopathology, molecular and cellular biology. 2002;70:47-54

22. Sahin U, Weskamp G, Kelly K, Zhou HM, Higashiyama S, Peschon J, et al. Distinct roles for adam10 and adam17 in ectodomain shedding of six egfr ligands. The Journal of cell biology. 2004;164:769-779

23. Choi SJ, Han JH, Roodman GD. Adam8: A novel osteoclast stimulating factor. Journal of bone and mineral research : the official journal of the American Society for Bone and Mineral Research. 2001;16:814-822

24. Kelly K, Hutchinson G, Nebenius-Oosthuizen D, Smith AJ, Bartsch JW, Horiuchi K, et al. Metalloprotease-disintegrin adam8: Expression analysis and targeted deletion in mice. Developmental dynamics : an official publication of the American 
Association of Anatomists. 2005;232:221-231

25. Mahoney ET, Benton RL, Maddie MA, Whittemore SR, Hagg T. Adam8 is selectively up-regulated in endothelial cells and is associated with angiogenesis after spinal cord injury in adult mice. The Journal of comparative neurology. 2009;512:243-255 


\section{Chapter 4}

\section{Myeloid ADAM10 deficiency modulates atherosclerotic plaque composition by shifting the balance from inflammation toward fibrosis}

Emiel P.C. van der Vorst, Mike Jeurissen, Ine M.J. Wolfs, Anke Keijbeck, Kosta Theodorou, Erwin Wijnands, Leon Schurgers, Silvio Weber, Marion J. Gijbels, Anouk A.J. Hamers, Daniela Dreymueller, Stefan Rose-John, Menno P.J. de Winther, Andreas Ludwig, Paul Saftig, Erik A.L. Biessen, Marjo M.P.C. Donners.

American journal of pathology, 2015 Apr;185(4): 1145-55 


\section{Abstract}

A Disintegrin And Metalloprotease ADAM10 is a metalloprotease involved in cleavage of various cell surface molecules such as adhesion molecules, chemokines and growth factor receptors. Although we have previously shown an association of ADAM10 expression with atherosclerotic plaque progression, a causal role of ADAM10 in atherosclerosis has not been investigated so far.

Bone marrow from conditional knockout mice lacking ADAM10 in the myeloid lineage or from littermate controls was transplanted into lethally irradiated $\mathrm{LDLr}^{-/}$mice on an atherogenic diet. Myeloid ADAM10 deficiency did not affect plaque size, but increased plaque collagen content. Matrix metalloproteinase-9 and -13 expression and matrix metalloproteinase- 2 gelatinase activity was significantly impaired in ADAM10-deficient macrophages, whereas their capacity to stimulate collagen production was unchanged. Furthermore, relative macrophage content in advanced atherosclerotic lesions was decreased. In vitro, ADAM10-deficient macrophages showed reduced migration toward monocyte chemoattractant protein-1 and transmigration through collagen. Additionally, ADAM10-deficient macrophages displayed increased anti-inflammatory phenotype with elevated interleukin-10, and reduced production of pro-inflammatory tumor necrosis factor, interleukin-12 and nitric oxide production in response to lipopolysaccharide.

These data suggest a critical role of ADAM10 for leukocyte recruitment, inflammatory mediator production and extracellular matrix degradation. Thereby, myeloid ADAM10 may play a causal role in modulating atherosclerotic plaque stability. 


\section{Introduction}

A Disintegrin and Metalloprotease 10 (ADAM10) is a ubiquitously expressed enzyme that can cleave extracellular domains of various cell surface molecules (a process called shedding), e.g. adhesion molecules, cytokine/ chemokine and growth factor receptors. ADAM10 mediated cleavage of specific substrates has been associated with various diseases, ranging from a protective function in Alzheimer disease to a detrimental role in rheumatoid arthritis and cancer 1,2. A crucial role for ADAM10 in development of the cardiovascular system is evident from the embryonic lethality of classical ADAM10-- mice, which were seen to display overt cardiac and vascular malformations ${ }^{3}$. Conditional deletion of ADAM10 in endothelial cells resulted either in embryonic lethality ${ }^{4}$ or in viable mice with vascular abnormalities and increased pathological neovascularization in a model of oxygen-induced retinopathy ${ }^{5}$. In agreement, we previously showed an important role of ADAM10 in VEGF-induced endothelial cell functions, e.g. regulating vascular permeability by cleaving VE-cadherin and migration of both endothelial cells and monocytes ${ }^{6}$. These processes are not only critically involved in angiogenesis, but also in atherosclerosis, the main underlying pathology of cardiovascular diseases.

Atherosclerosis is a chronic inflammatory disease characterized by the accumulation of lipids, extracellular matrix and inflammatory cells in the vessel wall. Although ADAM10 is implicated in various processes that are crucially involved in atherosclerosis ${ }^{7}$, like leukocyte adhesion and transmigration, the causal role of ADAM10 in cardiovascular diseases has not been investigated so far. Interestingly, we were the first to demonstrate ADAM10 expression in human atherosclerotic lesions, associated with plaque neovascularization and disease progression ${ }^{6}$. Besides neovascularization, the progression and stability of atherosclerotic lesions is strongly influenced by the balance between pro- and anti-inflammatory cytokines, thereby determining clinical outcome $^{8}$. In the atherosclerotic plaque, macrophages are the predominant inflammatory cell-type and crucial determinants of the inflammatory equilibrium ${ }^{9}$. Since we found high expression of ADAM10 in plaque macrophages ${ }^{6}$, we hypothesize that macrophage ADAM10 is crucially involved in inducing plaque development and progression. 
In this study, we therefore examined the role of ADAM10 in atherosclerotic plaque development by crossing ADAM10-floxed mice with the LysMcre-deleter strain ${ }^{10}$ to induce a myeloid specific deletion of ADAM10. Transplantation of ADAM10-LysMcre bone marrow into atherogenic $\mathrm{LDLr}^{-/}$mice showed that, although total plaque size was not affected, myeloid ADAM10 deficiency increased features of atherosclerotic plaque stability in these mice by increasing collagen and decreasing macrophage content of plaques. 


\section{Methods}

\section{Generation and characterization of ADAM10 fl/filLysM-cre ${ }^{+/ 0}$ mice}

The ADAM10 floxflox mice ${ }^{11}$ were generously donated by P. Saftig, crossed with LysM-cre mice ${ }^{10}$ and backcrossed to C57BI6 background for at least 6 generations. Mice were analyzed both macroscopically and microscopically for general organ morphology, including lungs, heart, gastro-intestinal tract, liver, pancreas, brain, skin, urinary and reproductive systems, spleen, lymph nodes, thymus, thyroid, and salivary and adrenal glands. For all experiments

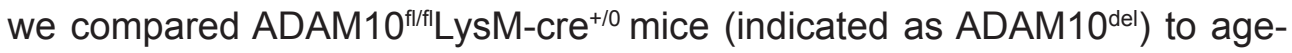
and sex-matched ADAM10 $0^{\mathrm{fl} / \mathrm{f}} \mathrm{LysM}-\mathrm{cre}^{0 / 0}$ littermate controls (ADAM10 ${ }^{\mathrm{wt}}$ ). All animal experiments were approved by the Animal Ethics Committee of the Maastricht University (permit number 2008-017).

\section{Bone marrow derived macrophage isolation and culture}

Bone marrow cells were isolated from femurs and tibiae of either wildtype or ADAM10 del mice. Cells were cultured in RPMI-1640 (GIBCO Invitrogen, Breda, the Netherlands) with 10\% heat-inactivated fetal calf serum (Bodinco B.V. Alkmaar, the Netherlands), penicillin $(100 \mathrm{U} / \mathrm{ml})$, streptomycin $(100 \mu \mathrm{g} /$ $\mathrm{ml}$ ) and L-glutamine $2 \mathrm{mM}$ (all GIBCO Invitrogen, Breda, the Netherlands) supplemented with $15 \%$ L929-conditioned medium (LCM) for 8-9 days to generate bone marrow-derived macrophages, as described previously ${ }^{12}$. Macrophages were seeded at 350000 cells per well in 24 wells plates and

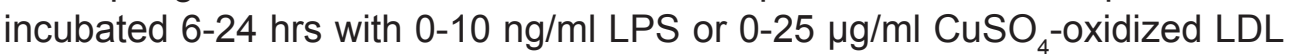
(oxLDL) for 24 hrs. IL-12, IL-10 and TNF- $\alpha$ ELISA assays (all Invitrogen) were performed on conditioned medium according to manufacturer's instructions. Analysis was performed using a micro-plate reader (Bio-Rad) at $450 \mathrm{~nm}$. NO production was measured in conditioned medium using Griess reagent (2.5\% $\mathrm{H}_{3} \mathrm{PO}_{4}, 1 \%$ sulfanilamide, $0.1 \%$ naphthalene diamine dihydrochloride). Analysis was performed using a micro-plate reader (Bio-Rad) at $550 \mathrm{~nm}$.

\section{Western blot}

Cell pellets of bone marrow derived macrophages from ADAM10 $10^{\text {th }}$ and ADAM10 $10^{\text {del }}$ mice were resuspended and homogenized in equal volumes of lysis buffer (1\% Triton, protease inhibitor, $150 \mathrm{mM} \mathrm{NaCl}, 200 \mathrm{mM}$ Tris and glycerol). Equal amounts of protein were separated on a $10 \%$ SDS-PAGE gel by electrophoresis and transferred to a nitrocellulose membrane. Membranes were probed with primary anti-ADAM10 antibody (1:500, generous gift from S. 
Rose-John) followed by secondary donkey anti-rabbit-peroxidase $(1: 10,000)$ antibody. Chemiluminescence was detected using a digital scanner.

\section{Bone marrow transplantation}

Ten to twelve week old male ADAM10 del or ADAM10 $1{ }^{\text {wt }}$ mice were used as donor mice ( $n=5$ per group). Forty female $L D L r^{--}$recipient mice (10-12 weeks) backcrossed onto a C57BI6 background for more than 10 generations were obtained from in-house breeding. Bone marrow transplantations were performed as described elsewhere ${ }^{13}$. In short, LDLr $^{-1}$ mice were lethally irradiated with 6Gy the day before and the day of transplantation. Mice were transplanted with $5 \times 10^{6}$ bone marrow cells isolated from wildtype or ADAM10 $10^{\text {del }}$ mice. After recovery for five weeks after transplantation mice were given a Western-type diet (WTD), containing $16 \%$ fat and $0.15 \%$ cholesterol (Hope Farms, The Netherlands). Before western type diet feeding, mice were fasted for $4 \mathrm{hrs}$, after which blood samples were drawn from the tail vein for analysis of plasma lipids and chimerism. Additional blood analysis was performed at 5 and 10 weeks of WTD. The chimerism in transplanted mice was determined on DNA from blood leukocytes as described previously ${ }^{13}$.

\section{Morphometry and immunohistochemistry of murine tissues}

After 10 weeks of WTD feeding, mice were anesthetized and euthanized. Mouse hearts were dissected and snap frozen in OCT. Atherosclerosis development (lesion size and progression stage) was determined as previously described ${ }^{14}$, with a slight modification in plaque classification. Plaques were classified as early (foam cell rich, but lacking a necrotic core), moderately advanced (containing a fibrotic cap and often a necrotic core, but no medial macrophage infiltration) and advanced lesions, typified by medial macrophage infiltrates, elastic lamina degradation and more pronounced necrosis and fibrosis. Serial sections $(7 \mu \mathrm{m})$ of the aortic root were cut and stained with toluidine blue for morphometric analysis and routine qualitative examination of collagen content, necrosis, foam cell content and amount of inflammatory cells (using scores from 0 (absent) to 3 (high abundance)). Moma2 (an antibody recognizing monocytes/ macrophages; gift from G. Kraal), NIMP-1 (in house cultured and purified antibody against neutrophils), TUNEL (Roche Diagnostics, Mannheim, Germany) and Sirius Red staining was used for the detection of monocytes/macrophages, neutrophils, apoptotic cells and collagen respectively. Suppl. Fig. 1 illustrates the region of interest used in all our plaque analyses, distinguishing plaque from media and aortic valve leaflets. A polarization filter and birefringence color discrimination 
was used to differentiate various collagen structures (ranging from loosely patched, immature, thin collagen (green) to tightly packed, mature, thick collagen fibers (red)) as described by MacKenna et al. ${ }^{15}$.

\section{Lipid analysis}

Mice were fasted for $4 \mathrm{hrs}$, before blood was drawn for plasma lipid analyses. Total plasma cholesterol and triglyceride levels as well as cholesterol in lipoprotein fractions were determined using standard enzymatic kits according to manufacturer's protocols (Sigma-Aldrich, Zwijndrecht, the Netherlands).

\section{Flow cytometry analysis (FACS)}

Absolute circulating leukocyte subset numbers were determined by flow cytometry calibrated using Trucount Beads (BD, New Jersey, U.S.). Leukocytes were defined as CD45+, $\mathrm{T}^{-}$-cells as $\mathrm{CD} 45^{+} \mathrm{CD} 3^{+} \mathrm{NK} 1.1^{-}$, NK cells as $\mathrm{CD}_{45^{+}} \mathrm{CD} 3^{-} \mathrm{NK}^{-1.1^{+}}$, B cells as CD45+ CD3- NK1.1- B220 ${ }^{+}$, granulocytes as $\mathrm{CD}^{+} 5^{+} \mathrm{CD}^{-} \mathrm{NK} 1.1^{-} \mathrm{B} 220^{-} \mathrm{CD} 11 \mathrm{~b}^{+} \mathrm{Ly}^{-} \mathrm{G}^{+}$and monocytes as CD45 ${ }^{+}$ CD3- NK1.1- B220- CD11 b+ Ly6G-. Inflammatory and resident monocytes were defined as Ly6 $C^{\text {high }}$ and Ly6C-$^{-}$, respectively, T-helper cells as CD45+ $\mathrm{CD}^{+} \mathrm{CD}^{+}$, cytotoxic $\mathrm{T}$-cells as $\mathrm{CD} 45^{+} \mathrm{CD} 3^{+} \mathrm{CD} 8^{+}$and regulatory $\mathrm{T}$-cells as $\mathrm{CD}^{+} \mathrm{CD}^{+} \mathrm{CD}^{+} 5^{+} \mathrm{Foxp}^{+}$. As a measure of peritoneal macrophage polarity, intracellular IL-10 and IL-12 production was assessed by flow cytometry, 6 hrs after activation by $10 \mathrm{ng} / \mathrm{ml}$ lipopolysaccharide (LPS) in the presence of GolgiSTOP (1:2000, BD-Pharmingen) and subsequent permeabilization. CD19- $\mathrm{F} 4 / 80^{+}$cells were considered macrophages. ADAM10 cell surface expression levels on BMDMs was measured by flow cytometry and expressed as geomean fluorescence intensity. A list of applied antibodies is given in Table 1. 
Table 1. List of antibodies used for flow cytometry

\begin{tabular}{|l||l||l|}
\hline \multicolumn{1}{|c|}{ Antigen } & \multicolumn{1}{c|}{ Clone } & \multicolumn{1}{c|}{ Company } \\
\hline CD16/32 & 93 & eBioscience \\
\hline CD45 & 30-F11 & Biolegend \\
\hline CD3e & 145-2c11 & eBioscience \\
\hline NK1.1 & PK136 & BD \\
\hline \hline Ly6G & 1A8 & BD \\
\hline CD11b & M1/70 & BD \\
\hline Ly6C & 1G7.G10 & Miltenyi \\
\hline CD4 & GK1.5 & BD \\
\hline \hline CD8 & $53-6.7$ & eBioscience \\
\hline \hline CD25 & PC61.5 & eBioscience \\
\hline \hline Foxp3 & FJK-16s & eBioscience \\
\hline B220 & RA3-6B2 & BD \\
\hline F4/80 & Cl:A31 & AbD \\
\hline CD19 & 1D3 & eBioscience \\
\hline \hline IL10 & Jes5-16E3 & eBioscience \\
\hline \hline IL12 & C17.8 & eBioscience \\
\hline ADAM10 & 139712 & R\&D Systems \\
\hline
\end{tabular}

\section{Vascular Smooth Muscle Cell isolation and culture}

Primary mouse vascular smooth muscle cells (SMCs) were isolated by collagenase and elastase (both Sigma-Aldrich, Zwijndrecht, the Netherlands) digestion of freshly dissected C57BI6 mouse aortas. Cells were cultured on laminin (1 $\mathrm{mg} / \mathrm{ml}$ ) coated wells (Sigma-Aldrich, Zwijndrecht, the Netherlands) in DMEM (GIBCO Invitrogen, Breda, the Netherlands) with 10\% heatinactivated fetal calf serum (Bodinco B.V. Alkmaar, the Netherlands), penicillin $(100 \mathrm{U} / \mathrm{ml})$, streptomycin $(100 \mu \mathrm{g} / \mathrm{ml})$ and L-glutamine $2 \mathrm{mM}$ (all GIBCO Invitrogen, Breda, the Netherlands). Cells were used for experiment between passage 6-8.

\section{Collagen assay in vascular Smooth Muscle Cells}

SMCs were plated as 50,000 cells per well. After overnight attachment, cells were starved (0\% FCS) for $48 \mathrm{hrs}$ and then incubated with macrophage 
supernatants for $24 \mathrm{hrs}$. Collagen production was measured by, fixating the cells in $3.7 \%$ formaldehyde, staining with $1 \%$ Sirius Red in $0.01 \mathrm{M} \mathrm{HCl}$, and lysis with $0.01 \mathrm{M} \mathrm{NaOH}$, as described previously ${ }^{16}$. Analysis was performed using a micro-plate reader (Bio-Rad) at $544 \mathrm{~nm}$, using a gelatin standard.

\section{Gene expression}

RNA was isolated with the High Pure RNA Isolation Kit (Roche, Basel, Switzerland). $500 \mathrm{ng}$ total RNA was reverse transcribed using the iScript cDNA Synthesis Kit (BioRad, Veenendaal, the Netherlands). Quantitative PCR was performed using $10 \mathrm{ng}$ cDNA, $300 \mathrm{nM}$ of each primer, and SensiMix (Quantace-Bioline, London, UK) in a total volume of $20 \mu \mathrm{l}$. All gene expression levels were corrected for cyclophilin A, $\beta$-actin and GAPDH as housekeeping genes. Primer sequences are available upon request.

\section{Chemotaxis and transmigration assays}

For chemotaxis and transmigration experiments, 24-well Costar transwell chambers (Corning, New York, U.S.) were used with $8 \mu \mathrm{M}$ pore size polycarbonate membranes. For transmigration, membranes were coated with rat tail collagen type I (Millipore). Upper wells were filled with 100 $\mu \mathrm{l}$ of cell suspension ( $2 \times 10^{5} \mathrm{BMDM}$ in $\left.100 \mu \mathrm{l} \mathrm{RPMI} / 0.2 \% \mathrm{BSA}\right)$. Lower wells contained $600 \mu \mathrm{l}$ RPMI/0.2\% BSA with $3 \mathrm{nM}$ murine MCP-1. After incubation for $2 \mathrm{hrs}$ (chemotaxis) or $4 \mathrm{hrs}$ (transmigration), migrated cells were quantified. Migrated cells were lyzed with $0.05 \%$ Triton in PBS, and endogenous $\beta$-glucoronidase activity was measured using $5 \mu \mathrm{M} p$-nitropheny$\beta$-D-glucoronide (Calbiochem, San Diego, U.S.) as a substrate. The number of transmigrated cells was determined using a serially diluted standard of defined cell number run in parallel. After $24 \mathrm{hrs}$, analysis was performed using a micro-plate reader at $405 \mathrm{~nm}$.

\section{Gelatin zymography}

MMP-2 enzymatic activity in ADAM10 wt and ADAM10 del BMDMs was determined by SDS-PAGE gelatin zymography. Cells were washed twice with PBS and lyzed in ice-cold NP-40 lysis buffer $(50 \mathrm{nM}$ Tris-HCl ph7.4, $100 \mathrm{mM} \mathrm{NaCl}, 10 \mathrm{mM} \mathrm{NaF}, 1 \mathrm{mM} \mathrm{Na3PO} 4,10 \%$ glycerol, 1\% nonidet). After $10 \mathrm{~min}$ incubation on ice, lysates were collected and $100 \mu \mathrm{g}$ of protein was applied to a $12 \%$ SDS-PAGE containing $0.1 \%(\mathrm{w} / \mathrm{v})$ gelatin (Biorad) in the presence of a non-reducing sample buffer $(62.5 \mathrm{mM}$ Tris- $\mathrm{HCl} \mathrm{pH} 6.8,10 \%$ SDS, $25 \%$ Glycerol, $0.01 \%$ Bromophenol Blue). Gels were incubated with $2.5 \%$ Triton X-100 (Sigma) for $30 \mathrm{~min}$ at room temperature and MMP-2 was 
activated overnight at $37^{\circ} \mathrm{C}$ in a developing buffer containing $50 \mathrm{mM}$ Tris$\mathrm{HCl}$ ( $\mathrm{pH} 7.5$ ), $200 \mathrm{mM} \mathrm{NaCl}, 5 \mathrm{mM} \mathrm{CaCl} 2,0.02 \%$ Brij-35. Thereafter, the gels were stained overnight at room temperature with PAGE-blue protein staining solution (Fisher Scientific) and destained with milliQ to detect proteolysis as a white band on a blue background.

\section{Statistical analysis}

Data are presented as mean \pm the standard error of the mean (SEM). All statistical analyses were performed using the Prism program (GraphPad Software Inc, San Diego, U.S.). Differences between treatment groups were evaluated for statistical significance with two-tailed, unpaired Student's t-test. Significance was accepted at the level of $p<0.05$. ${ }^{*} p<0.05 ;{ }^{* *} p<0.01$; ${ }^{* * *} p<0.001$. 


\section{Results}

\section{Generation and characterization of ADAM10-LysMcre mice}

To examine the role of myeloid ADAM10 in macrophage functions and atherosclerosis, ADAM10 floxed mice ${ }^{11}$ were crossed to mice that express cre under the control of the Lysozyme M (LysM) promoter to drive expression in the myeloid lineage, i.e. granulocytes and macrophages ${ }^{10}$. ADAM10 $0^{\text {ff/fli }}$ LysM-Cre $^{+/ 0}$ (ADAM10 ${ }^{\text {del }}$ ) mice were viable and fertile and no gross abnormalities were found ingeneral organ morphology both at macroscopic and microscopic level. FACS analyses did not reveal any major effects of myeloid ADAM10 deficiency on myeloid or lymphoid cell populations in bone marrow, blood, spleen and lymph nodes under baseline conditions (data not shown).

Deletion of ADAM10 could be confirmed by genomic PCR ${ }^{17}$ in the myeloid lineage of ADAM10 del mice, i.e. splenic granulocytes, splenic monocytes and peritoneal macrophages (Fig. 1A). Consistently, ADAM10 mRNA expression was significantly reduced in circulating granulocytes, in peritoneal and bone marrow derived macrophages (BMDMs), and to a lesser extent in monocytes but not in T cells (Fig. 1B). Within the monocyte population only partial deletion was observed, most likely because LysM expression in circulating monocytes is not sufficient to obtain the Cre levels for recombination and deletion of the floxed gene as described by Goren et al. ${ }^{18}$. Western blotting of BMDM lysates showed expression of inactive pro-ADAM10 ( 100 kD) and mature, catalytically active ADAM10 ( 70 kD, Suppl. Fig. 2A) in ADAM10 ${ }^{\text {wt }}$. Both forms were significantly reduced in ADAM10 del BMDMs. Additionally, FACS analysis of cell surface ADAM10 expression (i.e. mature ADAM10) confirmed this deletion (85\%, Fig. 1C). Moreover, ADAM10 del BMDMs released significantly $22 \%$ less soluble CXCL16, a known substrate of ADAM10 ${ }^{19}$, indicating loss of ADAM10 sheddase function after conditional deletion (Fig. 1D). To some extent the closely related metalloprotease ADAM17, which has partially overlapping substrates, could compensate for the loss of ADAM10. However, mRNA expression of ADAM17 does not show any compensatory effects in macrophages from ADAM10 del mice (Suppl. Fig. 2B). 
A

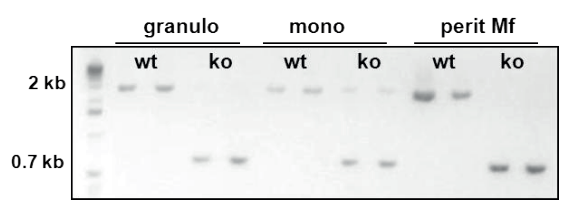

C

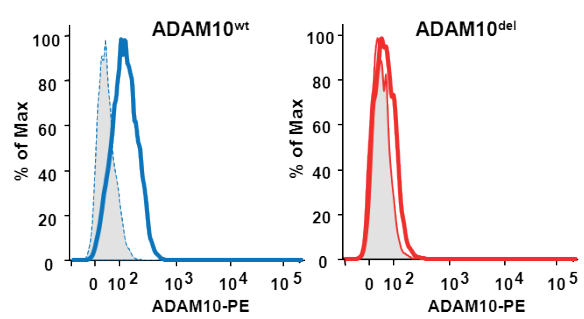

B
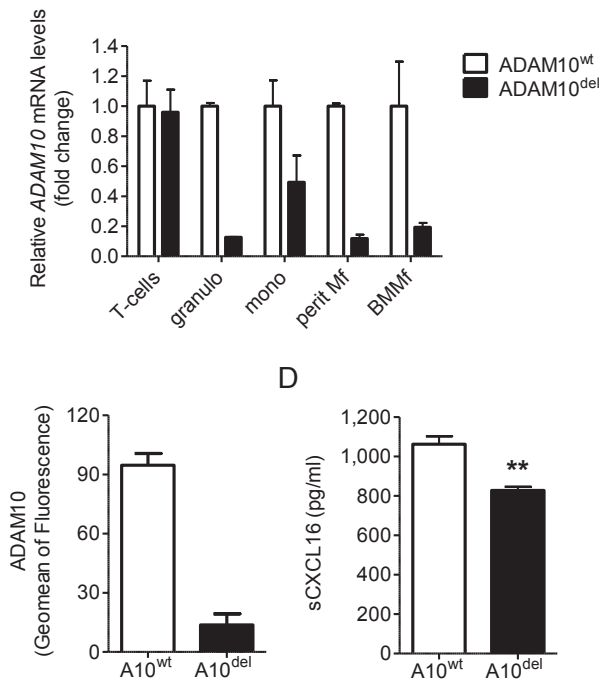

D

Figure 1. Characterization of ADAM10-LysMcre mice. Genomic PCR of ADAM10 (wt: 2 kb, ko: $0.7 \mathrm{~kb}$ ) constructs in spleen granulocytes and monocytes, and peritoneal macrophages $(\mathbf{A})$. ADAM10 mRNA expression in peritoneal macrophages, bone marrow-derived macrophages (BMDMs) and in circulating granulocytes, monocytes and T-cells of $A 10^{\text {wt }}$ and $A 10^{\text {del }}$ mice ( $n=2 /$ group, isolated by FACS sorting) (B). FACS analysis of ADAM10 in BMDMs (C, n=3/ group) and SCXCL16 levels in unstimulated BMDM supernatant (D, n=6/group).

\section{ADAM10 deficiency impairs macrophage inflammatory responses to LPS}

Since macrophages are the main inflammatory cell-type in atherosclerotic lesions, regulating the plaque inflammatory milieu by producing various pro- and anti-inflammatory mediators, we examined the role of ADAM10 in cytokine production by BMDMs after activation by either LPS or oxLDL. Whereas oxLDL-induced cytokine gene expression was essentially similar between ADAM10 ${ }^{\text {del }}$ and ADAM10 $1{ }^{\text {wt }}$ BMDMs (data not shown), production of the pro-inflammatory mediators IL-12, TNF and NO in response to LPS were reduced in ADAM10 del BMDMs compared to ADAM10 ${ }^{\text {wt }}$ (Fig. 2A-C), whereas anti-inflammatory IL-10 production was increased (Fig. 2D). These data may suggest polarization toward alternatively activated macrophages. 
A

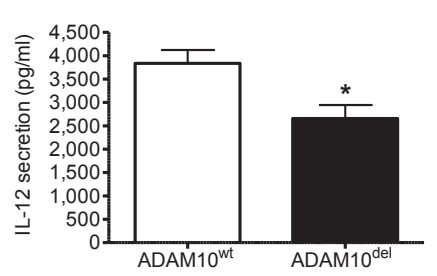

C

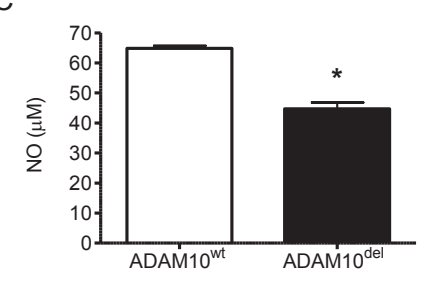

B
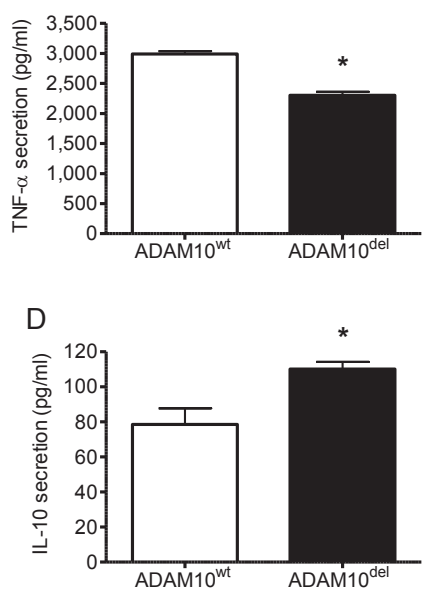

Figure 2. Impaired inflammatory responses in ADAM10 deficient BMDMs. Proinflammatory mediator (A-C) and anti-inflammatory cytokine (D) production by bone marrowderived macrophages (BMDMs) after activation by LPS (6 hrs for TNF and IL-10; 24 hrs for IL-12 and NO); n=3/group.

Systemic inflammatory effects of myeloid ADAM10 deficiency in LDLr-- mice $^{-1}$

Previously we showed ADAM10 expression associated with human atherosclerotic plaque progression ${ }^{6}$. However, a causal role for ADAM10 in atherosclerosis development has not yet been confirmed. Considering the key role of ADAM10 in regulating various inflammatory processes implicated in atherosclerosis ${ }^{7}$, its high expression in plaque macrophages ${ }^{6}$ and role in modulating macrophage inflammatory responses (Fig. 2), we investigated the effect of myeloid deficiency of ADAM10 on atherosclerosis development. Therefore, we reconstituted lethally irradiated $\mathrm{LDLr}^{-/}$mice with bone marrow from ADAM10 del mice or their wildtype littermate controls (ADAM10 ${ }^{\text {wt }}$ ). Five weeks after reconstitution, mice were placed on Western-type diet (WTD) for 10 weeks after which atherosclerosis development was assessed in the aortic root. Overall chimerism determination showed $96 \%$ repopulation for both genotypes. No differences between groups were found in body weight $\left(21.6 \pm 0.4 \mathrm{~g}\right.$ for ADAM10 ${ }^{\mathrm{wt}} \mathrm{vs} 21.5 \pm 0.5 \mathrm{~g}$ for ADAM10 $10^{\text {del }}$ transplanted mice $)$. 
Plasma levels of soluble IL-6R, a well-known in vivo substrate for ADAM10 albeit not myeloid-specific ${ }^{20}$, appeared to be $13 \%$ reduced in ADAM10 del transplanted mice, confirming functional ADAM10 deletion in these transplanted animals (Suppl. Fig. 3A).

Plasma cholesterol levels before or after feeding a WTD were not different between ADAM10 del and ADAM10 $1{ }^{\text {wt }}$ transplanted mice. Triglyceride levels were reduced in ADAM10 $10^{\text {del }}$ transplanted mice before and after 5 weeks of WTD feeding ( $25 \%$ and $37 \%$ respectively), whereas no significant differences were found after 10 weeks of WTD, when mice were sacrificed and atherosclerotic lesions were examined (Suppl. Fig. 3B-C).

Next, we analyzed by flow cytometry circulating leukocyte subset patterns in ADAM $10^{\text {del }}$ and ADAM $10^{\text {wt }}$ transplanted animals after 0,5 and 10 weeks of WTD. Though total leukocyte numbers, including both lymphoid and myeloid lineages, decreased between 0 and 5-10 weeks of WTD, which might be partly due to repopulation dynamics after transplantation, we found no differences in number of total CD45+ leukocytes or lymphoid lineages between genotypes (data not shown). Interestingly, the number of $L y 6 G^{+} C D 11 b^{+}$granulocytes was significantly decreased in ADAM10 $10^{\text {del }}$ transplanted mice, both at baseline and after 10 weeks of WTD feeding (26\% and $21 \%$ respectively, Fig. 3A-C and H). Whereas the total number of Ly6G-CD11 $\mathrm{b}^{+}$monocytes was not different, analysis of monocyte subsets revealed that the relative amount of Ly6C hi ('inflammatory') monocytes was significantly increased in ADAM $10^{\text {del }}$ transplanted mice, both at 5 and 10 weeks of WTD feeding $(20 \%$ and $26 \%$ respectively, Fig. 3D-G and I-L, Suppl. Fig. 4). Tissue resident macrophages in the peritoneum, however, did not reflect a more proinflammatory phenotype and even tended to display increased production of anti-inflammatory IL-10 cytokine (Suppl. Fig. 5), as also described above for BMDMs (Fig. 2D). 
A

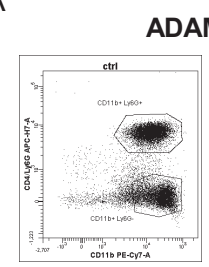

ADAM10wt

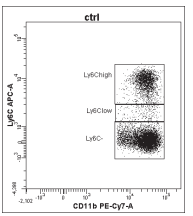

B

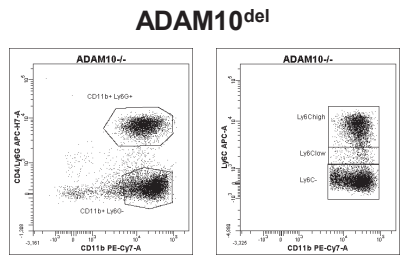

C
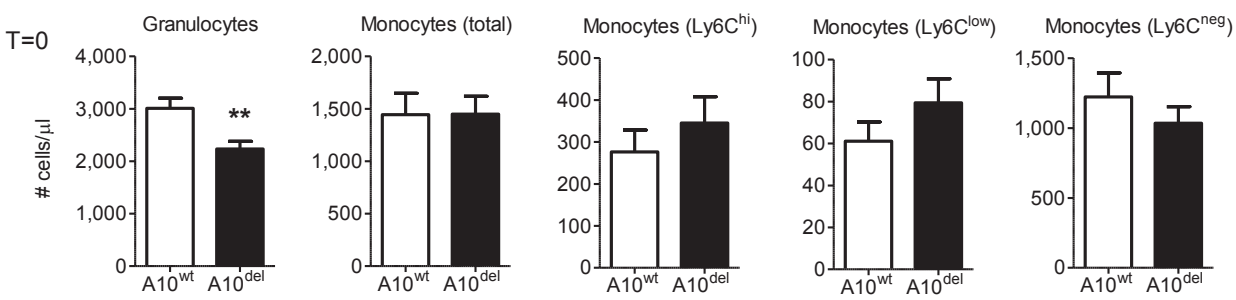

D
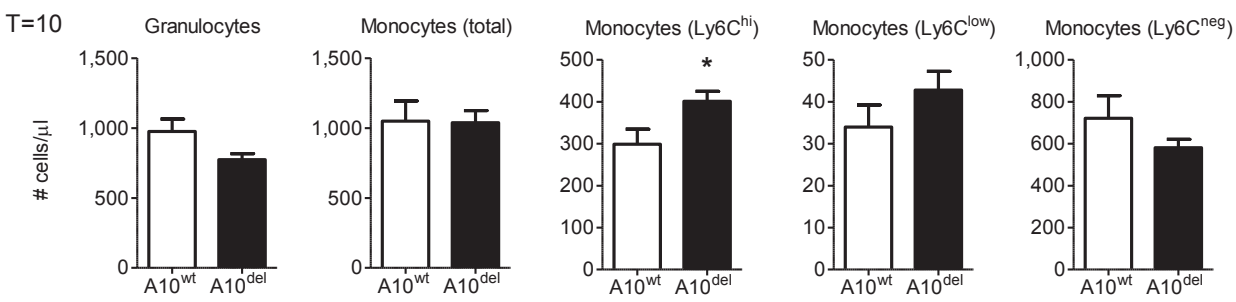

Figure 3. Reduced granulocyte levels and increased Ly6C ${ }^{\text {hi }}$ monocyte levels in ADAM10 del transplanted mice. Representative FACS plots showing granulocyte and monocyte (sub) populations (A-B) and absolute numbers of circulating Ly6 $\mathrm{G}^{+}$granulocytes, $\mathrm{Ly} 6 \mathrm{C}^{+}$monocytes, Ly6C ${ }^{\text {hi }}$ 'inflammatory', Ly6C ${ }^{\text {low }}$ and Ly6C $C^{\text {neg }}$ 'patrolling' monocytes in blood of ADAM10 ${ }^{\text {wt }}$ and ADAM10 del transplanted $\mathrm{LDLr}^{-/}$mice at $\mathrm{t}=0$ weeks $(\mathbf{C})$ or $\mathrm{t}=10$ weeks $(\mathbf{D})$ of Western-type diet feeding; $n=10 /$ group. 
Myeloid deficiency of ADAM10 does not affect atherosclerotic lesion size, but increases features of plaque stability in LDLr ${ }^{-/}$mice

Analysis of atherosclerotic lesions in the aortic root revealed neither differences in lesion area (Fig. 4A-C), nor in lesion progression between ADAM10 del and ADAM10 wt transplanted mice (Fig. 4D). Atherosclerotic lesions were further examined by routine pathological examination for their composition, i.e. collagen content, necrosis, foam cell content, amount of inflammatory cells. Whereas plaque necrosis and foam cell content as well as adventitial inflammatory cell content did not differ between groups, a significant increase in plaque fibrosis was found (Fig. 4E, $p<0.05$ ).

A

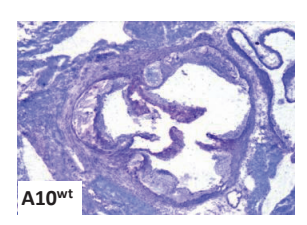

B

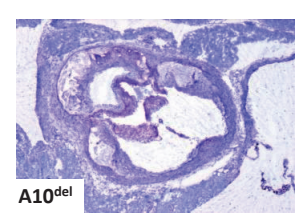

D

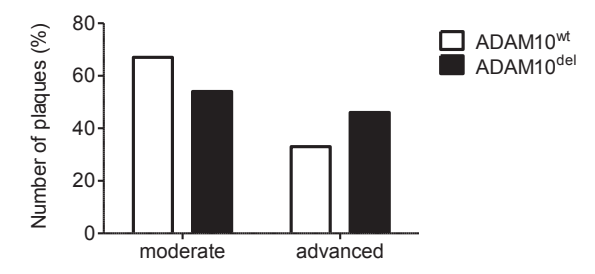

C

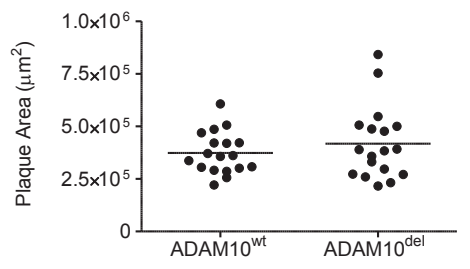

E

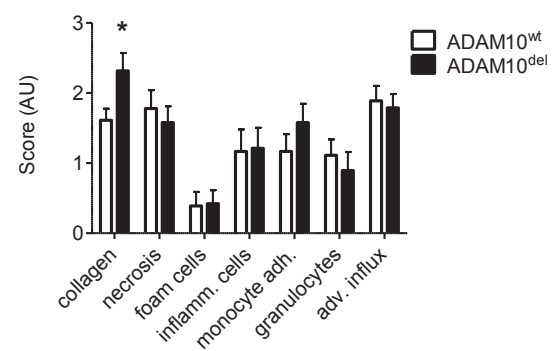

Figure 4. Myeloid ADAM10 deficiency does not affect atherosclerotic lesion size. Representative pictures of toluidine blue-stained sections of the aortic root of ADAM10 $1{ }^{\text {wt }}(\mathbf{A})$ and $\operatorname{ADAM} 10^{\mathrm{del}}(\mathrm{B})$ transplanted mice, magnification 40x. Quantification of plaque area (C), plaque classification (D) and routine pathological examination of plaque composition (E); n=18/group. 
This increase in plaque fibrosis observed by routine pathological examination was quantified by Sirius red staining and showed a marked $40.5 \%$ increase in collagen content of ADAM10 del transplanted mice compared to ADAM10 $10^{\text {wt }}$ (Fig. 5A-D, p<0.05). This effect appeared to be predominantly attributed to an increased collagen content in advanced atherosclerotic lesions (Fig. 5D, $\mathrm{P}<0.01$ ). Differentiating on the basis of collagen structure (loosely patched, immature, thin collagen (green) versus tightly packed, mature, thick collagen fibers (red)) by using a polarization filter, we were able to demonstrate that increased plaque fibrosis in ADAM10 del transplanted mice was due to an increase in mature, thick collagen fiber content (Fig. 5E-G, $p<0.05$ ).

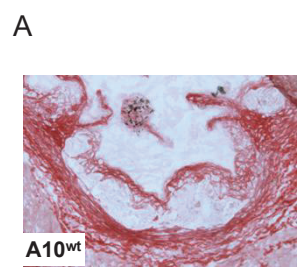

E

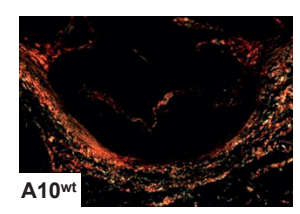

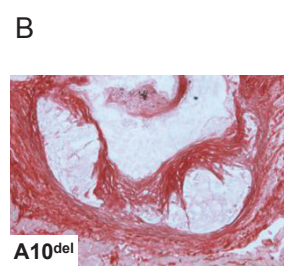

$\mathrm{F}$

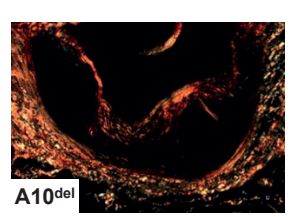

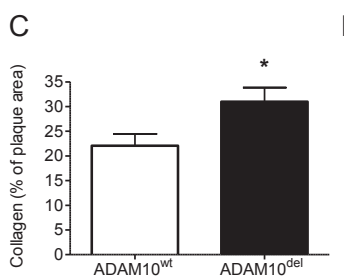

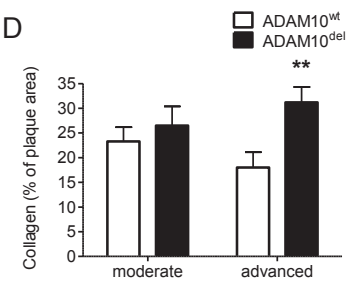

G

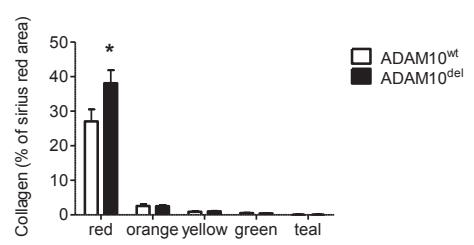

Figure 5. Increased mature, thick collagen fiber content in lesions from ADAM10 del transplanted mice. Representative pictures of Sirius-red stained collagen as visualized by normal light microscopy $(\mathbf{A}-\mathbf{B})$ and polarizing light microscopy (E-F) of ADAM10 1 wt $(A+E)$ and $A D A M 10^{\text {del }}(B+F)$ transplanted mice, magnification 40x. Quantification of total collagen in of ADAM10 $1{ }^{\text {wt }}$ and ADAM10 del transplanted mice (C-D), magnification 40x; n=15-17/group. Quantification of different collagen fibers in the plaques ranging from thick, mature collagen (red) to loosely packed, thin collagen fibers (green) (G); n=9-11/group. 
Increased plaque collagen can be either the result of increased production or decreased degradation of collagen. Quantitative real-time PCR analyses revealed that genes associated with collagen production, e.g. TGF $\beta$, collagen I, or arginase I, did not differ between ADAM10 del and ADAM10 $10^{\text {wt }}$ BMDMs (Suppl. Fig. 6). To examine whether myeloid ADAM10 deficiency could modulate collagen production by vascular smooth muscle cells (SMCs) via a paracrine mechanism, we incubated SMCs with conditioned medium of ADAM10 del compared to ADAM10 ${ }^{\text {wt }}$ BMDMs. Collagen I, III, IV or VIII gene expression (Suppl. Fig. 7A-D) as well as collagen synthesis (Suppl. Fig. 7E) by SMCs incubated with conditioned medium of ADAM10 del and ADAM10wt BMDMs was essentially similar. Though stimulation of collagen production by SMC seems not affected, myeloid ADAM10 deficiency may influence the amount of SMCs recruited to the atherosclerotic lesions. Quantification of alpha-smooth muscle actin (aSMA)-positive SMCs indeed showed an increase in SMC content in ADAM10 del plaques, although this difference was not statistically significant (Fig. 6A-B). Regarding collagen degradation, SMCs incubated with conditioned medium did not show any differences in expression of matrix degrading enzymes (Suppl. Fig. 7F-H), whereas mRNA expression of MMP-2, MMP-9 and MMP-13 in ADAM10 del BMDMs was reduced $(65 \%, 70 \%$ and $25 \%$, respectively in non-LPS stimulated cells, which was significant for MMP-9 and -13; Fig. 6C-E). To confirm the reduced MMP gene expression was accompanied by a reduction in matrix degrading activity, we performed gelatin zymography on BMDM lysates. Though activity levels were very low in these cells and even undetectable for MMP-9, ADAM $10^{\text {del }}$ BMDMs showed $25 \%$ reduced MMP-2 gelatinase activity (Fig. 6F). In summary, the increase in plaque fibrosis in ADAM10 del mice most likely results from a combination of increased SMC numbers and decreased matrix degradation. 
A

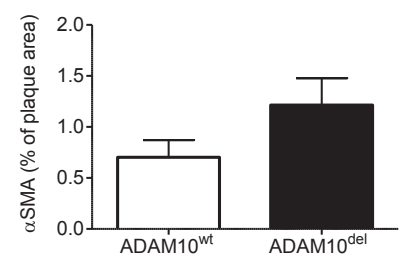

c

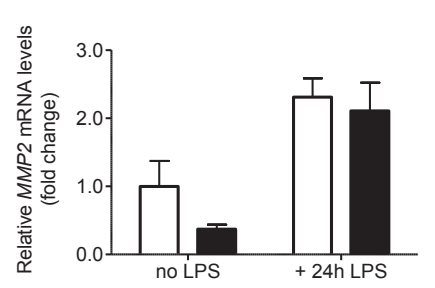

F

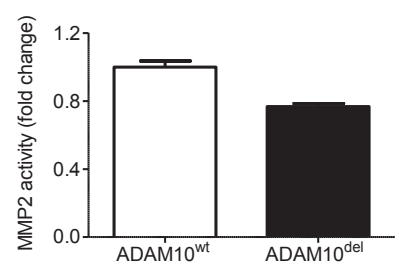

B

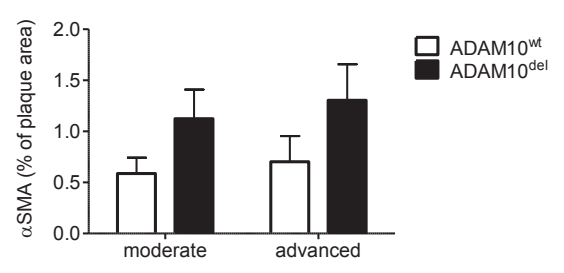

D
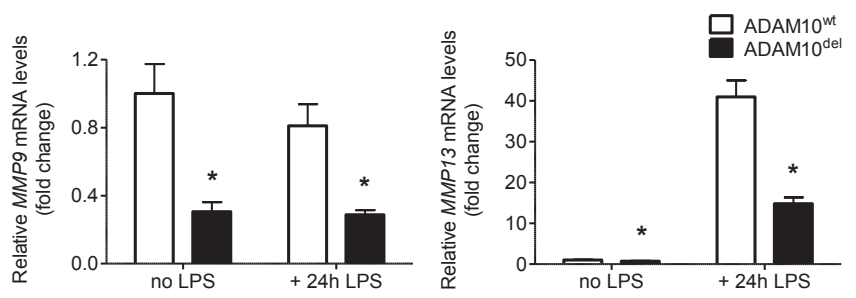

Figure 6. Increased plaque SMC numbers and decreased matrix degradation in macrophages from ADAM10 del transplanted mice. Quantification of $\alpha S_{M A}{ }^{+} S M C s$ of ADAM10 ${ }^{\text {wt }}$ and ADAM10 del transplanted mice (A-B), magnification 40x; $n=12-16 /$ group. Matrix metalloproteases-2 (C), -9 (D) and -13 (E) mRNA expression in ADAM10 ${ }^{\text {wt }}$ and ADAM10 del BMDMs after $24 \mathrm{hrs}$ stimulation with $0-10 \mathrm{ng} / \mathrm{ml}$ LPS in vitro; $\mathrm{n}=3 /$ group. Quantification of MMP-2 gelatinase activity of BMDMs $(\mathbf{F}, \mathrm{n}=2)$. 
To quantify differences in inflammatory cell content of the plaques, (immuno) histochemical staining was performed. Despite lowered circulating granulocyte levels, no differences were found in the amount of $\mathrm{NIMP}^{+}$plaque neutrophils (Fig. 7A-D). Although relative $\mathrm{MOMA}^{+}$macrophage content in moderate atherosclerotic lesions was not different, advanced atherosclerotic lesions of ADAM10 $10^{\text {del }}$ transplanted mice contained less macrophages compared to ADAM10 ${ }^{\text {wt }}$ transplanted mice (Fig. 7E-H, p<0.05). Since inflammatory cytokine production by ADAM10 del BMDMs in response to LPS suggested a more anti-inflammatory alternatively activated phenotype, we quantified iNOS $^{+}$(M1) and Dectin1+ (M2) macrophages but found no differences (Suppl. Fig. 8).

The amount of macrophages in the plaque is determined by the balance between recruitment and cell death. The relative apoptotic cell content in advanced plaques (number of TUNEL ${ }^{+}$cells per plaque area) was significantly decreased in ADAM10 del transplanted mice compared to ADAM10 ${ }^{\text {wt }}(38 \%$, Suppl. Fig. 9A-B, p<0.05); however, the number of $\mathrm{TUNEL}^{+}$cells per macrophage area did not change (data not shown), suggesting that the decreased $\mathrm{TUNEL}^{+}$cell content was merely a reflection of the decreased macrophage content. Furthermore, necrotic core content of the atherosclerotic lesions did not differ between groups (Suppl. Fig. 9C-D). As a measure of macrophage recruitment to the plaque, we determined monocyte adhesion at the luminal side of the lesions, showing a trend toward increased numbers of adherent monocytes in ADAM10 del transplanted mice (Suppl. Fig. 9E-F, $p=0.08$ ). Furthermore, we determined the effect of ADAM10-deficiency on macrophage MCP-1 induced chemotaxis and transmigration through collagen type I in vitro. We found both types of migration to be reduced in ADAM10 del BMDMs (Fig. 7I-J). Therefore the reduced macrophage content in advanced lesions of ADAM $10^{\text {del }}$ transplanted animals could, at least in part, be explained by a reduced migration capacity of ADAM10-deficient macrophages. On the other hand, a reduction in relative macrophage content may be a reflection of the increased fibrosis as indeed the absolute macrophage content was not different (Suppl. Fig. 10).

Altogether these data indicate that deficiency of ADAM10 dampens macrophage pro-inflammatory responses, decreases their matrix-degrading and migration capacity in vitro. In vivo, myeloid ADAM10 deficiency increased fibrosis and reduced relative macrophage content in the plaque, which suggests enhanced plaque stability. 
A

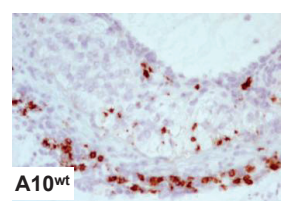

E

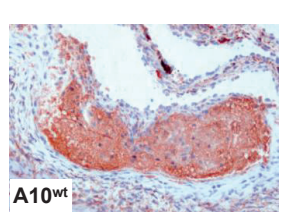

I

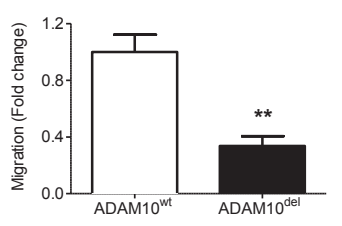

B

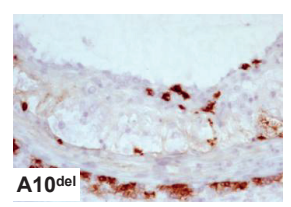

$\mathrm{F}$

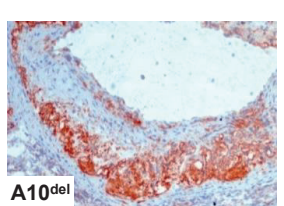

$J$
C

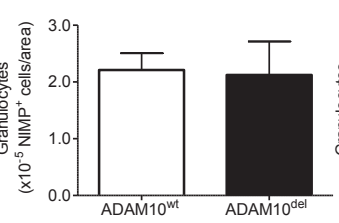

G

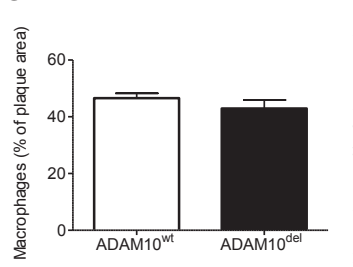

D

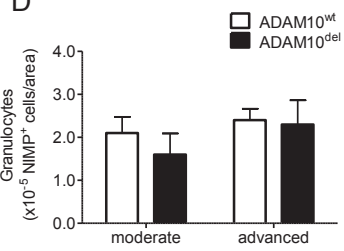

$\mathrm{H}$

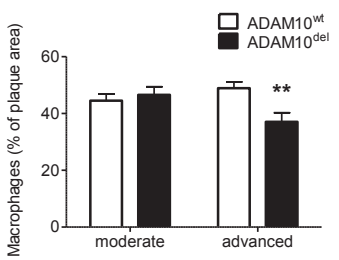

Figure 7. Reduced macrophage content in advanced lesions from ADAM10 ${ }^{\text {del }}$ transplanted mice and decreased macrophage migration capacity in vitro. Representative pictures and quantification of $\mathrm{NIMP}^{+}$neutrophils (A-D) and $\mathrm{MOMA}^{+}$macrophages (E-H) of ADAM10 ${ }^{\text {wt }}$ and ADAM10 del transplanted mice, magnification 40x; $n=15-17$ /group. MCP-1 induced chemotaxis (I) and MCP-1 induced transmigration through collagen-I (J) of ADAM10 wt and ADAM10 del BMDMs; $n=3$ /group. 


\section{Discussion}

In this study, we show a causal role of myeloid ADAM10 in atherosclerotic plaque development in $\mathrm{LDLr}^{-/}$chimeras. Although myeloid ADAM10 deficiency did not affect total plaque size, plaques of myeloid deficient ADAM10 chimeras displayed a more stable phenotype, with apparent increased extracellular matrix content and reduced macrophage accumulation.

Conceivably, the reduction in macrophage content in advanced plaques of ADAM10 del transplanted mice could be explained by a decreased monocyte/ macrophage recruitment, since ADAM10 has been implicated in the cleavage of various molecules important for chemotaxis, adhesion and (trans) migration, e.g. cadherins and selectins ${ }^{21}$. Interestingly, we found an increase in circulating 'inflammatory' Ly6C ${ }^{\text {hi }}$ monocytes in ADAM10 del transplanted mice, which could indicate a decrease in adhesion and migration to the vessel wall. Monocyte adhesion to the atherosclerotic lesion was not significantly altered between groups, and showed even a trend to increased monocyte adhesion. This modest effect is likely due to the fact that monocytes do not express sufficient lysozyme to obtain effective deletion of the floxed ADAM10 gene ${ }^{18}$. We found a reduction in macrophage MCP-1 induced migration and transmigration through collagen type I in vitro, which could at least partly explain the reduction in macrophage content in advanced lesions of ADAM10 $10^{\text {del }}$ transplanted mice. Similarly, we recently demonstrated reduced leukocyte infiltration into acutely inflamed lung in ADAM10-LysMcre mice 22. Various molecules important for leukocyte recruitment have been shown to be cleaved by ADAM10 including cadherins ${ }^{23}$ as well as CXCL16 and fractalkine, two chemokines (expressed not only in myeloid cells, but also in endothelium) that act as adhesion molecules in their membrane-bound form and chemotactic factors when soluble ${ }^{24,25}$. These chemokines are especially important in advanced atherosclerotic plaques ${ }^{26}$. On the other hand, we did not observe a difference in plaque neutrophil numbers in ADAM10 del transplanted mice, despite a significant reduction in circulating granulocytes. Considering its role in junctional/adhesion molecule cleavage in endothelial cells, regulating endothelial permeability and leukocyte recruitment it is conceivable that endothelial specific deletion of ADAM10 would have a stronger effect on atherogenesis. 
Besides reduced macrophage recruitment, the reduced macrophage content in advanced plaques of ADAM10 del transplanted mice could also be caused by increased macrophage apoptosis. Indeed ADAM10 has been implicated in the shedding of members of the death receptor family e.g. TNF, CD40L and FasL ${ }^{27,28}$ and ADAM10 deficiency could therefore lead to increased apoptosis. However, the number of $\mathrm{TUNEL}^{+}$cells per macrophage area was even reduced in advanced lesions, indicating that cell death does not explain the differences in macrophage content.

Myeloid ADAM10 deficiency considerably increased plaque fibrosis in ADAM $10^{\text {del }}$ transplanted mice, which could be related to the relative decrease in macrophage content. The plaque extracellular matrix content is determined by the balance between collagen production and degradation. Known inducers of collagen production in myeloid cells, especially in the 'wound healing' (M2) macrophage phenotype ${ }^{29}$, are for example arginase I, Fizz-1 and transforming growth factor TGF- $\beta$. Arginase is an enzyme that drives L-arginine metabolism toward ornithine and subsequently proline production, which is critical for collagen synthesis ${ }^{30}$. Although we did find ADAM10deficient BMDMs to produce less pro-inflammatory mediators like TNF, IL12 and NO but more anti-inflammatory cytokine IL-10 in response to LPS, suggesting a switch toward M2 macrophages, we could not find an increase in pro-fibrotic M2-markers such as arginase I, YM-1, Fizz-1 or TGF- $\beta$. Indeed ADAM10 del plaques did not show any differences in NNOS $^{+}$or Dectin-1 $1^{+}$ macrophages, markers of M1 or M2 macrophages, respectively, indicating that the ADAM10 deficiency in macrophages does not induce a general switch in polarization. In peritoneal macrophages isolated from the atherosclerotic mice, ADAM10-deficiency also resulted in a much milder, though still antiinflammatory response to LPS. This, however, does not necessarily reflect the situation in the atherosclerotic lesions, where other stimuli (e.g. oxLDL) may be more relevant. TNF is a well-known target of ADAM17 cleavage, although other proteases including ADAM10 are capable of releasing TNF ${ }^{27}$, and this may become important under pathologic conditions. However, it remains to be determined how ADAM10-deletion affects the expression of the other inflammatory mediators. It is conceivable though that this is an indirect effect of reduced TNF production in ADAM10-deficient macrophages (Fig. 2B). Moreover, ADAM10 ${ }^{\text {del }}$ macrophage conditioned medium did neither change expression nor production of collagen by vascular smooth muscle cells. Hence, the increased plaque fibrosis can probably not be attributed to a stimulated collagen production, though we found an (non-significant) 
increase in SMC content which could contribute to the increase in fibrosis. At the other side of the equation, ADAM $10^{\text {del }}$ macrophages displayed decreased expression of matrix metalloproteases (e.g. MMP-2, -9 and -13) and reduced MMP-2 gelatinase activity, responsible for the degradation of the extracellular matrix. Moreover, ADAM10 itself has been reported to have matrix-degrading capacity, albeit in a rather artificial in vitro setting ${ }^{31}$. Therefore it is conceivable that the increased collagen content in plaques of ADAM10 del transplanted mice is caused either directly or indirectly by decreased collagenase activity. Whether ADAM10 deficiency directly leads to decreased MMP activity or is an indirect effect e.g. by changing the macrophage phenotype, i.e. reducing TNF- $\alpha$, or integrin signaling (now modulated by the absence of the ADAM10 disintegrin domain) remains to be determined.

Besides myeloid cells, the role of ADAM10 in other vascular cells e.g. endothelial cells and smooth muscle cells in atherosclerosis can be investigated using other cre-deleter strains.

In conclusion, this study is the first to unveil a causal role of ADAM10 in atherosclerosis. Deletion of ADAM10 in the myeloid lineage did not affect plaque size, but increased plaque fibrosis and reduced macrophage accumulation. Consistently, in vitro production of inflammatory mediators, migratory as well as matrix-degrading capacity of BMDMs was reduced. By shifting the balance from inflammation towards more fibrosis, myeloid ADAM10 deficiency may enhance plaque stability. 


\section{Acknowledgements}

We thank Patrick van Gorp and Chantal Pöttgens for technical support.

This study was supported by Dr. E. Dekker post-doctoral grant nrs 2007T034 and 2012T079 from the Netherlands Heart Foundation (to Marjo M.P.C. Donners) and a grant from the Deutsche Forschungsgemeinschaft DFG, SFB877-TPA3 (to Paul Saftig and Silvio Weber). 


\section{References}

1. Crawford HC, Dempsey PJ, Brown G, Adam L, Moss ML. Adam10 as a therapeutic target for cancer and inflammation. Curr Pharm Des. 2009;15:2288-2299

2. Duffy MJ, McKiernan E, O'Donovan N, McGowan PM. The role of adams in disease pathophysiology. Clin Chim Acta. 2009;403:31-36

3. Hartmann D, de Strooper B, Serneels L, Craessaerts K, Herreman A, Annaert W, et al. The disintegrin/metalloprotease adam 10 is essential for notch signalling but not for alpha-secretase activity in fibroblasts. Hum Mol Genet. 2002;11:26152624

4. Zhang C, Tian L, Chi C, Wu X, Yang X, Han M, et al. Adam10 is essential for early embryonic cardiovascular development. Dev Dyn. 2010;239:2594-2602

5. Glomski K, Monette S, Manova K, De Strooper B, Saftig P, Blobel CP. Deletion of adam10 in endothelial cells leads to defects in organ-specific vascular structures. Blood. 2011;118:1163-1174

6. Donners MM, Wolfs IM, Olieslagers S, Mohammadi-Motahhari Z, Tchaikovski V, Heeneman $S$, et al. A disintegrin and metalloprotease 10 is a novel mediator of vascular endothelial growth factor-induced endothelial cell function in angiogenesis and is associated with atherosclerosis. Arterioscler Thromb Vasc Biol. 2010;30:2188-2195

7. van der Vorst EP, Keijbeck AA, de Winther MP, Donners MM. A disintegrin and metalloproteases: Molecular scissors in angiogenesis, inflammation and atherosclerosis. Atherosclerosis. 2012;224:302-308

8. Virmani R, Kolodgie FD, BurkeAP, FinnAV, Gold HK, TulenkoTN, etal. Atherosclerotic plaque progression and vulnerability to rupture: Angiogenesis as a source of intraplaque hemorrhage. Arterioscler Thromb Vasc Biol. 2005;25:2054-2061

9. Libby $\mathrm{P}$, Ridker PM, Maseri A. Inflammation and atherosclerosis. Circulation. 2002;105:1135-1143

10. Clausen BE, Burkhardt C, Reith W, Renkawitz R, Forster I. Conditional gene targeting in macrophages and granulocytes using lysmcre mice. Transgenic research. 1999;8:265-277

11. Jorissen E, Prox J, Bernreuther C, Weber S, Schwanbeck R, Serneels L, et al. The disintegrin/metalloproteinase adam10 is essential for the establishment of the brain cortex. The Journal of neuroscience : the official journal of the Society for Neuroscience. 2010;30:4833-4844

12. Kanters E, Gijbels MJ, van der Made I, Vergouwe MN, Heeringa P, Kraal G, et al. Hematopoietic nf-kappab1 deficiency results in small atherosclerotic lesions with an inflammatory phenotype. Blood. 2004;103:934-940 
13. Kanters E, Pasparakis M, Gijbels MJ, Vergouwe MN, Partouns-Hendriks I, Fijneman $\mathrm{RJ}$, et al. Inhibition of nf-kappab activation in macrophages increases atherosclerosis in Idl receptor-deficient mice. The Journal of clinical investigation. 2003;112:1176-1185

14. Gijbels MJ, van der Cammen M, van der Laan LJ, Emeis JJ, Havekes LM, Hofker $\mathrm{MH}$, et al. Progression and regression of atherosclerosis in apoe3-leiden transgenic mice: An immunohistochemical study. Atherosclerosis. 1999;143:1525

15. MacKenna DA, Omens JH, Covell JW. Left ventricular perimysial collagen fibers uncoil rather than stretch during diastolic filling. Basic research in cardiology. 1996;91:111-122

16. Rensing KL, de Jager SC, Stroes ES, Vos M, Twickler MT, Dallinga-Thie GM, et al. Akt2/ldlr double knockout mice display impaired glucose tolerance and develop more complex atherosclerotic plaques than Idlr knockout mice. Cardiovascular research. 2014;101:277-287

17. Weber S, Niessen MT, Prox J, Lullmann-Rauch R, Schmitz A, Schwanbeck R, et al. The disintegrin/metalloproteinase adam10 is essential for epidermal integrity and notch-mediated signaling. Development. 2011;138:495-505

18. Goren I, Allmann N, Yogev N, Schurmann C, Linke A, Holdener M, et al. A transgenic mouse model of inducible macrophage depletion: Effects of diphtheria toxindriven lysozyme m-specific cell lineage ablation on wound inflammatory, angiogenic, and contractive processes. The American journal of pathology. 2009;175:132-147

19. Ludwig A, Hundhausen C, Lambert MH, Broadway N, Andrews RC, Bickett DM, et al. Metalloproteinase inhibitors for the disintegrin-like metalloproteinases adam10 and adam17 that differentially block constitutive and phorbol esterinducible shedding of cell surface molecules. Combinatorial chemistry \& high throughput screening. 2005;8:161-171

20. Garbers C, Janner N, Chalaris A, Moss ML, Floss DM, Meyer D, et al. Species specificity of adam 10 and adam17 proteins in interleukin-6 (il-6) trans-signaling and novel role of adam10 in inducible il-6 receptor shedding. The Journal of biological chemistry. 2011;286:14804-14811

21. Reiss K, Saftig P. The "a disintegrin and metalloprotease" (adam) family of sheddases: Physiological and cellular functions. Semin Cell Dev Biol. 2009;20:126-137

22. Pruessmeyer J, Hess FM, Ahlert H, Groth E, Pasqualon T, Schwarz N, et al. Leukocytes require the metalloproteinase adam10 but not adam17 for cell migration and for inflammatory leukocyte recruitment into the alveolar space. Blood. 2014 
23. Reiss K, Maretzky T, Ludwig A, Tousseyn T, de Strooper B, Hartmann D, et al. Adam10 cleavage of $n$-cadherin and regulation of cell-cell adhesion and betacatenin nuclear signalling. EMBO J. 2005;24:742-752

24. Hundhausen C, Schulte A, Schulz B, Andrzejewski MG, Schwarz N, von Hundelshausen $P$, et al. Regulated shedding of transmembrane chemokines by the disintegrin and metalloproteinase 10 facilitates detachment of adherent leukocytes. J Immunol. 2007;178:8064-8072

25. Ludwig A, Weber C. Transmembrane chemokines: Versatile 'special agents' in vascular inflammation. Thrombosis and haemostasis. 2007;97:694-703

26. Cheng C, Tempel D, van Haperen R, de Boer HC, Segers D, Huisman M, et al. Shear stress-induced changes in atherosclerotic plaque composition are modulated by chemokines. The Journal of clinical investigation. 2007;117:616-626

27. Hikita A, Tanaka N, Yamane S, Ikeda Y, Furukawa H, Tohma S, et al. Involvement of a disintegrin and metalloproteinase 10 and 17 in shedding of tumor necrosis factor-alpha. Biochemistry and cell biology = Biochimie et biologie cellulaire . 2009;87:581-593

28. Schulte M, Reiss K, Lettau M, Maretzky T, Ludwig A, Hartmann D, et al. Adam10 regulates fasl cell surface expression and modulates fasl-induced cytotoxicity and activation-induced cell death. Cell Death Differ. 2007;14:1040-1049

29. Wolfs IM, Donners MM, de Winther MP. Differentiation factors and cytokines in the atherosclerotic plaque micro-environment as a trigger for macrophage polarisation. Thrombosis and haemostasis. 2011;106:763-771

30. Raes G, Van den Bergh R, De Baetselier P, Ghassabeh GH, Scotton C, Locati M, et al. Arginase-1 and ym1 are markers for murine, but not human, alternatively activated myeloid cells. Journal of immunology. 2005;174:6561; author reply 65616562

31. Millichip MI, Dallas DJ, Wu E, Dale S, McKie N. The metallo-disintegrin adam10 (madm) from bovine kidney has type iv collagenase activity in vitro. Biochemical and biophysical research communications. 1998;245:594-598 


\section{Supplemental Figures}

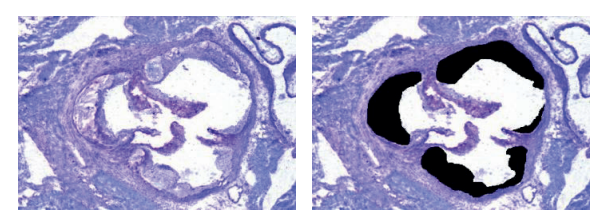

Supplemental Figure 1. Region of interest for lesion analyses. Toluidine stained cross sections of aortic heart valve with atherosclerotic lesion. The right panel illustrates the region of interest for plaque analyses (the black area). The internal elastic lamina is used as boundary between plaque and media, while the peripheral boundaries are determined by the sites where the aortic valves start.

A

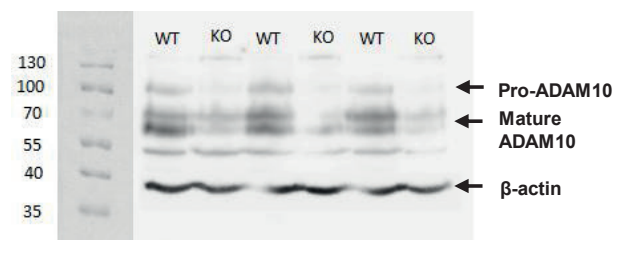

B

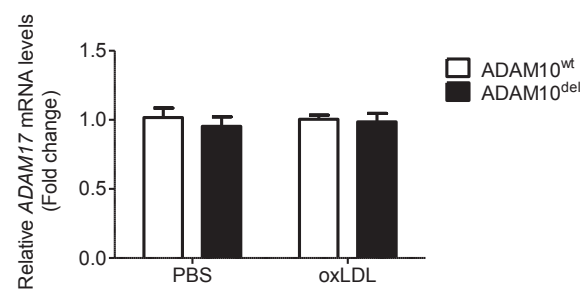

Supplemental Figure 2. Characterization of ADAM10-LysMcre mice. (A) Western blot of ADAM10 in BMDMs ( $n=3 /$ group). (B) ADAM17 mRNA levels of ADAM10 wt and ADAM10 del BMDMs stimulated with $0-25 \mu \mathrm{g} / \mathrm{ml}$ oxLDL for $24 \mathrm{hrs}$; $\mathrm{n}=3$ /group.

A

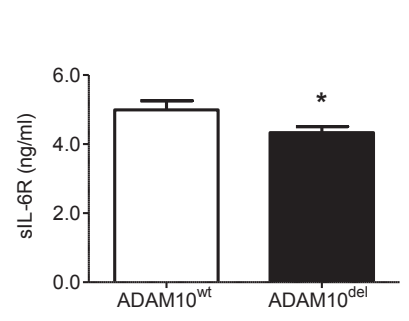

B

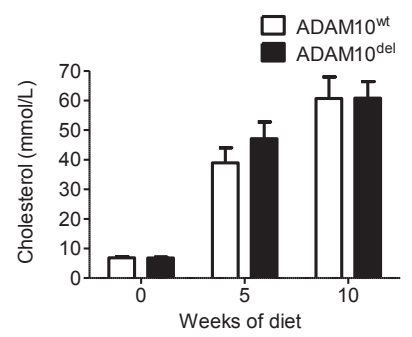

C

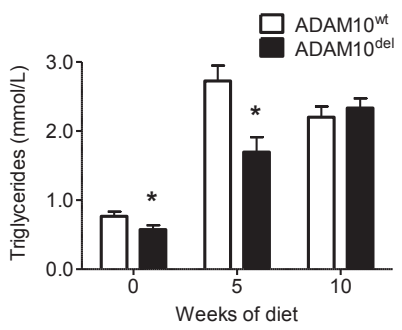

Supplemental Figure 3. Reduced soluble IL-6R and triglyceride plasma levels in ADAM10 del transplanted mice. (A) Soluble IL-6R levels in plasma of ADAM10 wt and ADAM10 ${ }^{\text {del }}$ transplanted LDLr ${ }^{-1}$ mice after 10 weeks of Western-type diet feeding. Plasma cholesterol (B) and triglyceride (C) levels of ADAM10 ${ }^{\text {wt }}$ and ADAM10 del transplanted LDL $r^{-1-}$ mice; $n=18$ /group. 


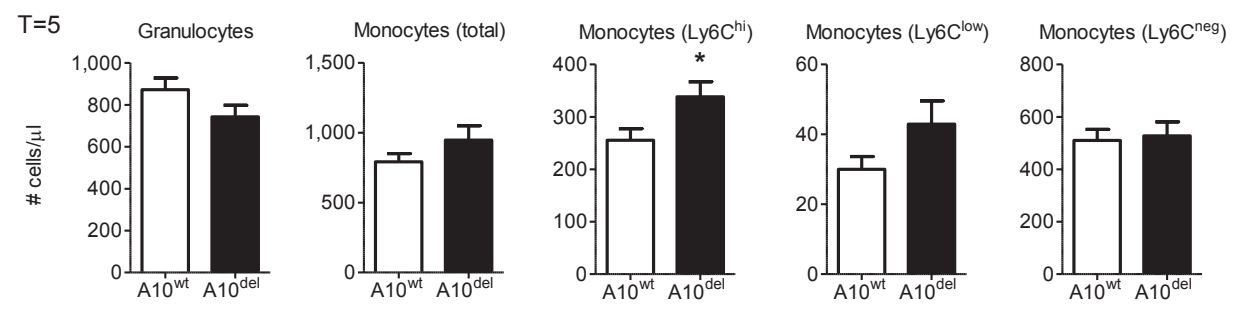

Supplemental Figure 4. Increased Ly6C ${ }^{\text {hi }}$ monocyte levels in ADAM10 del transplanted mice after 5 weeks WTD feeding. Absolute cell numbers of $\mathrm{Ly}_{6 \mathrm{G}} \mathrm{G}^{+}$granulocytes, $\mathrm{Ly} 6 \mathrm{C}^{+}$ monocytes, Ly6C hi 'inflammatory', Ly6C low and Ly6C- 'patrolling' monocytes in blood of ADAM $10^{\text {wt }}$ and ADAM10 del transplanted LDLr-- mice at $t=5$ weeks of Western-type diet feeding; n=10/group.

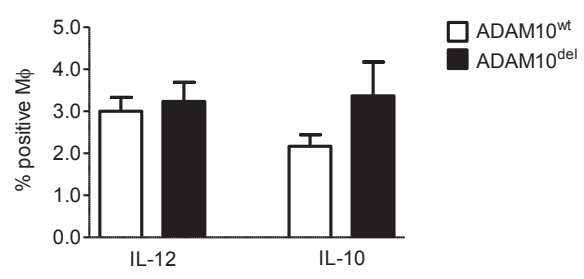

Supplemental Figure 5. No changes of inflammatory phenotype in peritoneal macrophages. Pro-inflammatory IL-12 and anti-inflammatory IL-10 cytokine production by peritoneal macrophages of ADAM10 $10^{\text {wt }}$ and ADAM10 del transplanted LDLr-l- mice after 10 weeks of Western-type diet feeding as determined by intracellular FACS analysis; $n=18 /$ group.

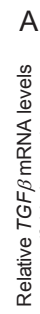

B

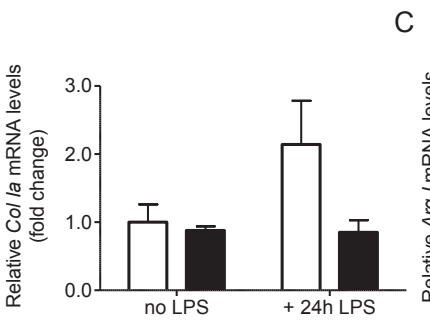

C
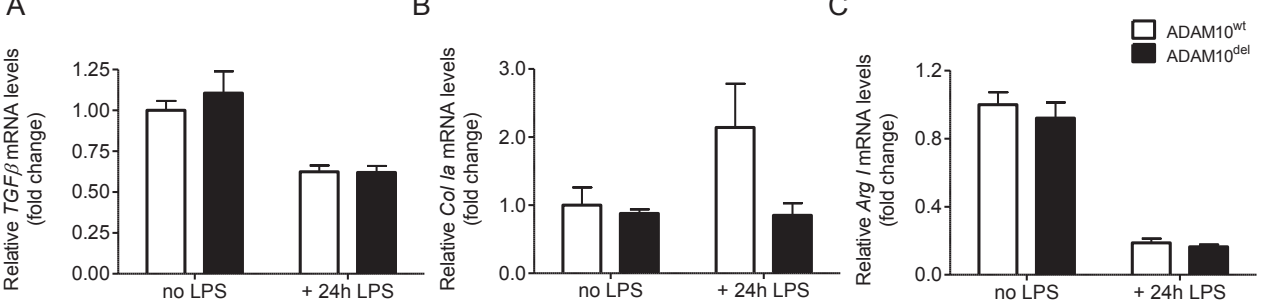

Supplemental Figure 6. No differences in expression of genes associated with collagen production. TGF $\beta$ (A), collagen I (B) and arginase I (C) mRNA expression in ADAM10 ${ }^{\text {wt }}$ and $A D A M 10^{\text {del }} B M D M s$ after $24 \mathrm{hrs}$ stimulation with $0-10 \mathrm{ng} / \mathrm{ml}$ LPS in vitro; $\mathrm{n}=3$. 

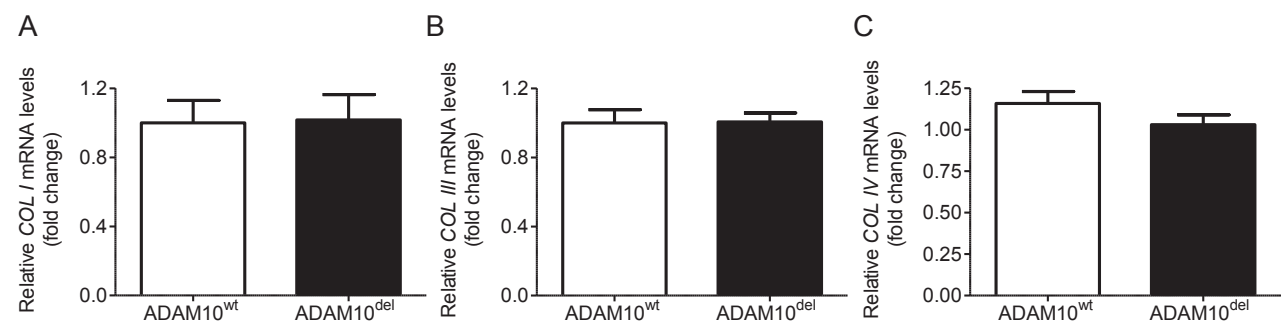

$\mathrm{D}$

$\mathrm{E}$
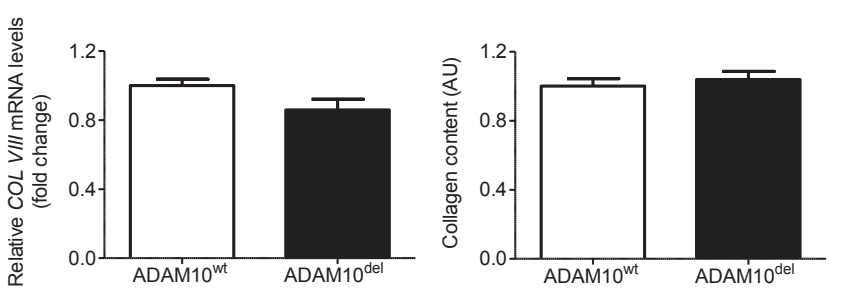

$\mathrm{F}$

G

$\mathrm{H}$
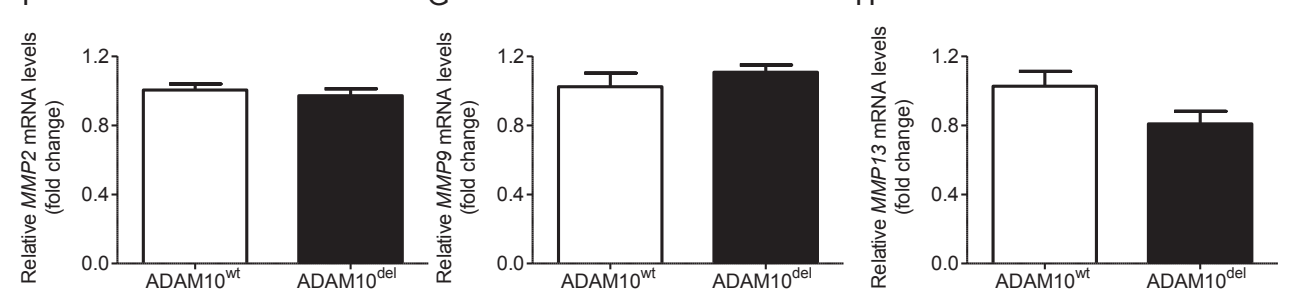

Supplemental Figure 7. Myeloid ADAM10 deficiency does not indirectly influence collagen production in SMCs. Collagen type I (A), III (B), IV (C) and VIII (D) mRNA expression, collagen protein production (E, detected by Sirius Red staining) or MMP-2 $(\mathbf{F})$, MMP-9 (G), MMP-13 (H) mRNA expression in vascular smooth muscle cells after $24 \mathrm{hrs}$ incubation with conditioned medium of ADAM10 ${ }^{\text {wt }}$ and ADAM10 $10^{\text {del }}$ BMDMs; $n=3-5 /$ group. 
A

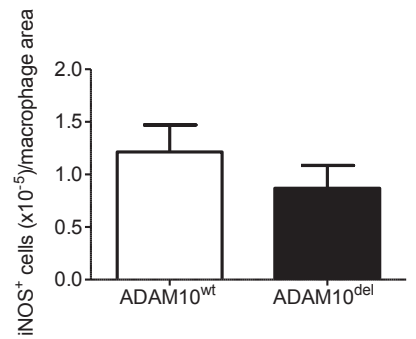

B

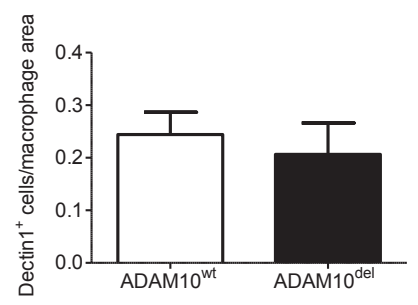

Supplemental Figure 8. Plaque macrophage polarization markers are not changed due to ADAM10 deficiency. Quantification of $(A)$ iNOS $^{+}(M 1)$ and (B) Dectin-1+ $(M 2)$ macrophages in atherosclerotic plaques of ADAM10 ${ }^{\text {wt }}$ and ADAM10 del transplanted mice; $n=17$ and 14-15, respectively.

A

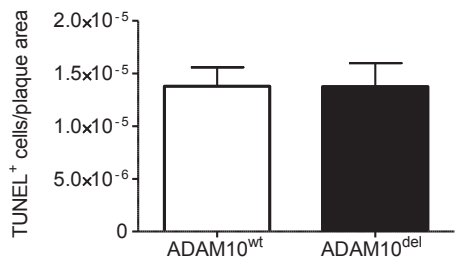

C

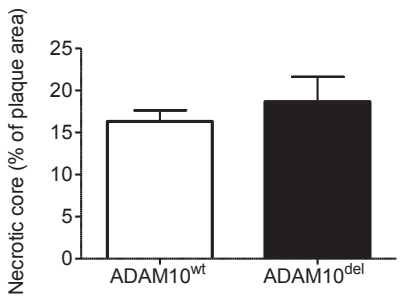

E

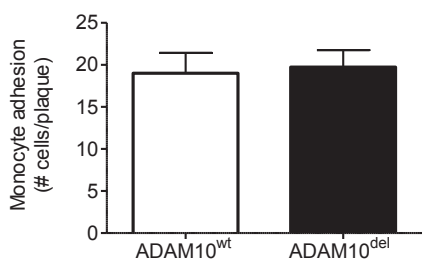

B

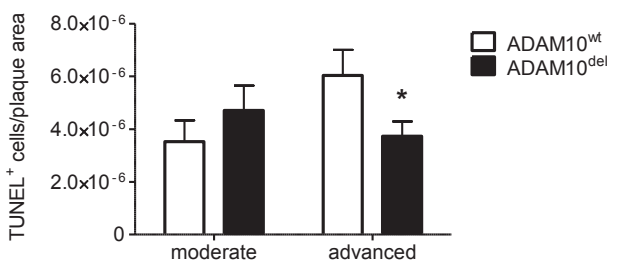

D

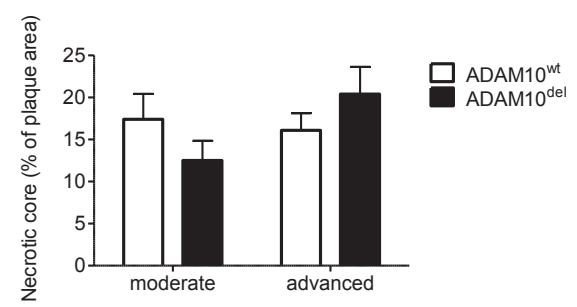

$\mathrm{F}$

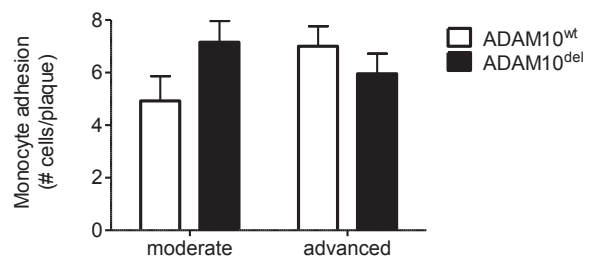


Supplemental Figure 9. No major changes on cell death or cell adhesion in ADAM10 del transplanted mice. Quantification of $\mathrm{TUNEL}^{+}$apoptotic cells in atherosclerotic plaques of ADAM10 ${ }^{\text {wt }}$ and ADAM10 del transplanted mice (A-B); $n=13$ and 16 respectively. Necrotic core content quantified on toluidine blue-stained sections of ADAM $10^{\text {wt }}$ and ADAM $10^{\text {del }}$ transplanted mice (C-D); $n=18$ /group. Monocyte adhesion to the atherosclerotic plaques of ADAM $10^{\text {wt }}$ and ADAM10 $10^{\text {del }}$ transplanted mice as quantified on toluidine blue-stained sections (E-F); $n=15$ and 16 respectively.
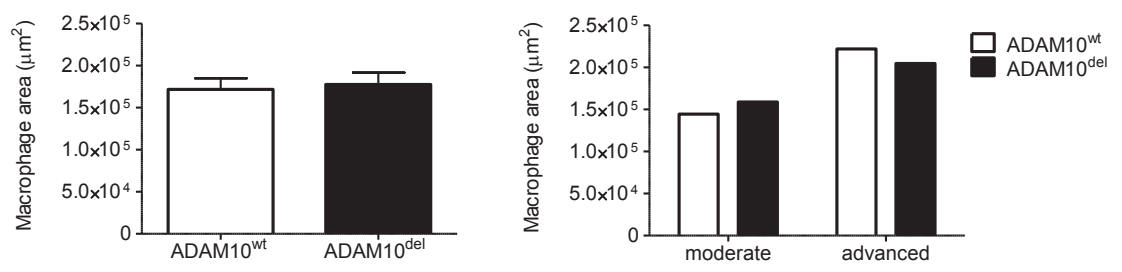

Supplemental Figure 10. Absolute plaque macrophage content is not changed due to myeloid ADAM10 deficiency. Absolute amount of $\mathrm{MOMA}^{+}$macrophages of atherosclerotic plaques of ADAM10 $10^{\text {wt }}$ and ADAM10 del transplanted mice, $n=15-17 /$ group. 


\section{Chapter 5}

\section{Endothelial ADAM10 deficiency modulates leukocyte recruitment and remarkably augments atherosclerotic lesion development}

Emiel P.C. van der Vorst, Timo Rademakers, Kosta Theodorou, Marijn Brilman, Jaap van Buul, Thomas L. Theelen, Erwin Wijnands, Marion J. Gijbels, Leon Schurgers, Mat Rousch, Sofie M. Walenbergh, Michael Lehrke, Corinna Lebherz, Dominique de Kleijn, Siu Kwan Sze, Daniela Dreymueller, Jacob Bentzon, Andreas Ludwig, Erik A.L. Biessen, Marjo M.P.C. Donners

In preparation 


\section{Abstract}

A Disintegrin And Metalloprotease (ADAM) 10 has been associated with atherosclerotic lesion development, the main cause of cardiovascular diseases. Atherosclerosis is characterized by the accumulation of lipids and inflammatory cells in the vessel wall. Key steps in this pathology are the adhesion and transmigration of leukocytes to/through the endothelium. We performed a proteomics analysis of supernatant from mouse endothelial cells with or without ADAM10 inhibition, revealing that ADAM10 is a crucial regulator in this process by shedding transmembrane proteins, like adhesion molecules but also chemokines. We further investigated the causal role of endothelial ADAM10 in atherosclerosis development. Surprisingly, endothelial ADAM10 deficiency resulted in a significantly increased plaque area $(45 \%)$ in aortic root and brachiocephalic arteries, while no differences were observed in plasma cholesterol or various circulating leukocyte subpopulations. Atherosclerotic lesions from endothelial ADAM10 deficient mice were also more advanced, characterized by more plaque necrosis and relatively less plaque macrophages. In vivo imaging, showed a clear trend toward increased leukocyte adhesion and transmigration. Interestingly, in line with our proteomics data, ADAM10 deficient mice had lower plasma levels of Annexin A5. In conclusion, endothelial ADAM10 protects against atherosclerosis, though further research is needed to fully elucidate the underlying mechanisms. 


\section{Introduction}

Cardiovascular disease (CVD), like myocardial infarction and stroke, is a major health burden and the leading cause of death worldwide ${ }^{1}$. The main underlying cause of CVD is atherosclerosis, which is a chronic inflammatory disease characterized by the accumulation of lipids, inflammatory cells and cellular debris in the vessel wall ${ }^{2}$. The onset of atherosclerosis is marked by arterial endothelium damage and subsequent increase in endothelial permeability, allowing lipoproteins to infiltrate into and accumulate in the vessel wall ${ }^{3}$. In parallel, circulating leukocytes are attracted to the site of injury by the expression of a range of adhesion molecules such as ICAM1 and VCAM1 and the secretion of various chemokines, such as CXCL16 and $\mathrm{CX}_{3} \mathrm{CL} 1$ by endothelial cells ${ }^{1,4}$. Upon adhesion these cells may transmigrate through the endothelial layer into the intima and contribute to lesion development ${ }^{5,6}$.

Various chemokines, adhesion molecules and junction molecules are substrates of A Disintegrin And Metalloproteases (ADAMs), a family of proteolytic enzymes expressed on all vascular cells, including endothelial cells ${ }^{7,8}$. ADAM10 is one of the major enzymes of this family and has been associated with atherosclerosis development and progression, based on increased expression of ADAM10 upon human lesion development 9. However, a causal role for ADAM10 in atherosclerosis remains to be established. A large amount of substrates of ADAM10 are already described, many of which being important in inflammatory processes related to atherosclerosis, like tumor necrosis factor- $\alpha$ (TNF- $\alpha$ ), its receptor TNF-R and the chemokines CXCL16 and $\mathrm{CX}_{3} \mathrm{CL} 1{ }^{10,11}$. The latter two are membrane bound chemokines that primarily act as adhesion molecules, while they can be released as soluble chemokines through shedding by ADAM10 ${ }^{11}$. Additionally, ADAM10 influences endothelial permeability by cleaving VECadherin, a major endothelial junction molecule ${ }^{9,12}$. Considering the key role of ADAM10 in regulating adhesion molecule expression, chemokine secretion and cell permeability ${ }^{1,8}$ and that endothelial cells are instrumental in atherosclerosis, we investigated the effect of endothelial deficiency of ADAM10 on atherosclerosis development. 
Contrary to our expectations endothelial ADAM10 deficiency appears to results in significantly increased rather than decreased plaque area and progression. Lesions from endothelial ADAM10 deficient mice showed more necrosis, while their macrophage content was decreased. In line with the plaque phenotype, endothelial permeability, leukocyte adhesion and transmigration as assessed by in vivo two-photon laser scanning microscopy imaging tended to be enhanced. 


\section{Methods}

\section{Cell culture and proteomic analysis}

Mouse endothelial bEND5 cells were cultured in DMEM medium (GIBCO Invitrogen) supplemented with $10 \%$ (vol/vol) heat-inactivated fetal calf serum (Bodinco B.V.), $100 \mathrm{U} / \mathrm{ml}$ penicillin, $100 \mu \mathrm{g} / \mathrm{ml}$ streptomycin (All GIBCO Invitrogen). Cells were incubated in serum free medium with or without GI254023X for $24 \mathrm{hrs}$. Conditioned medium was harvested and concentrated using $3 \mathrm{kD}$ Vivaspin concentrators (Sartorius). Equal volumes were separated on a $10 \%$ SDS-PAGE gel by electrophoresis and subsequently stained with Coomassie blue staining. Gels were cut in $1 \mathrm{~mm}^{2}$ sections and freeze dried overnight. Secretome analysis was performed using liquid chromatographytandem mass spectrometry.

\section{Mice}

For all experiments we compared ADAM10 $1 / / f / T i e 2-\mathrm{cre}^{+/ 0}$ mice (from here on indicated as ADAM10 $\left.{ }^{\text {del }}\right)$ to age- and sex-matched ADAM10 $0^{\mathrm{fl} / \mathrm{fT}} \mathrm{Tie} 2-\mathrm{cre}^{0 / 0}$ littermate controls (ADAM10 ${ }^{\mathrm{wt}}$ ). All mice (mixed genetic background) were injected intravenously with $10^{9}$ particles of the adeno-associated virus serotype 8 (AAV8) containing D374Y-human proprotein convertase subtilisin/ kexin type 9 (PCSK9) ${ }^{13}$. Adeno-associated virus will target the liver and induce overexpression of PCSK9, which will degrade LDL receptors (LDLr) ${ }^{13,14}$. The thus created functional LDLr knockdown will render the mice hyperlipidemic and prone to atherosclerosis. AAV8-PCSK9 treated/infected mice were fed a Western type diet (WTD) consisting of $16 \%$ fat and $0.15 \%$ cholesterol (Special diet services) for 12 weeks. For blood analysis at baseline and at 6 and 12 weeks of WTD feeding, mice were fasted for $4 \mathrm{hrs}$, after which blood samples were drawn from the tail vein for analysis of plasma lipids, leukocyte subset patterns and cytokines. All animal experiments were approved by the Animal Ethics Committee of the Maastricht University (permit number 2013009).

\section{Morphometry and immunohistochemistry of murine tissue}

After 12 weeks of WTD feeding, mice were anesthetized and euthanized. Mouse hearts were dissected, fixed in $1 \%$ paraformaldehyde and embedded in paraffin. Size and progression stage of atherosclerotic lesions was determined as previously described ${ }^{2}$, with a slight modification in plaque classification. Plaques were classified as early (foam cell rich, but lacking a necrotic core), 
moderately advanced (containing a fibrotic cap and often a necrotic core, but no medial macrophage infiltration) and advanced lesions, typified by medial macrophage infiltrates, elastic lamina degradation and more pronounced necrosis and fibrosis. Serial sections $(7 \mu \mathrm{m})$ of the aortic root were cut and stained with haematoxyline-eosine for morphometric analysis and routine qualitative examination of collagen content, necrosis, foam cell content and amount of inflammatory cells (using scores from 0 (absent) to 3 (high abundance)). MAC3 (an antibody recognizing macrophages), Ly6G (antibody against neutrophils) and Sirius Red staining was used for the detection of macrophages, neutrophils and collagen respectively. A polarization filter and birefringence color discrimination was used to differentiate various collagen structures (ranging from loosely patched, immature, thin collagen (green) to tightly packed, mature, thick collagen fibers (red)) as described by MacKenna et al ${ }^{15}$. For evaluation of liver inflammation MAC1 (an antibody recognizing macrophages) and NIMP-1 (neutrophil specific antibody directed against Ly6G; gift from P. Heeringa) were used.

\section{Lipid analysis}

Total plasma cholesterol and triglyceride levels as well as cholesterol in lipoprotein fractions were determined, under fasting conditions, using standard enzymatic kits according to manufacturer's protocols (DiaSys Diagnostic Systems GmbH, Germany).

\section{Flow cytometry analysis}

Absolute circulating leukocyte subset numbers were determined by flow cytometry calibrated using Trucount Beads (BD, New Jersey, U.S.) after 0,6 and 12 weeks WTD. Leukocytes were defined as CD45 ${ }^{+}$, T-cells as $\mathrm{CD}_{4}{ }^{+} \mathrm{CD}^{+} \mathrm{NK} 1.1^{-}$, NK cells as CD45+ ${ }^{+} \mathrm{CD} 3^{-} \mathrm{NK} 1.1^{+}$, B cells as CD45+ CD3NK1.1- B220 $^{+}$, granulocytes as CD45 ${ }^{+}$CD3- NK1.1- B220- CD11 b ${ }^{+}$Ly6G $^{+}$ and monocytes as CD45 ${ }^{+}$CD3- NK1.1- B220- CD11 b ${ }^{+}$Ly6G- A list of applied antibodies is given in Table 1. 
Table 1. List of antibodies used for flow cytometry

\begin{tabular}{|l|l||l|}
\hline \multicolumn{1}{|c|}{ Antigen } & \multicolumn{1}{c|}{ Clone } & \multicolumn{1}{c|}{ Company } \\
\hline CD16/32 & 93 & eBioscience \\
\hline CD45 & $30-\mathrm{F} 11$ & Biolegend \\
\hline CD3e & 145-2c11 & eBioscience \\
\hline NK1.1 & PK136 & BD \\
\hline Ly6G & 1A8 & BD \\
\hline CD11b & M1/70 & BD \\
\hline B220 & RA3-6B2 & BD \\
\hline
\end{tabular}

\section{In vivo multiphoton laser scanning microscopy (MPLSM)}

At sacrifice, 5 mice per genotype were used for in vivo imaging. The mice were first anaesthetized using ketamine $(100 \mathrm{mg} / \mathrm{kg}$ body weight, Nematek) and xylazine $(10 \mathrm{mg} / \mathrm{kg}$ body weight, Sedamun) and the carotid artery as well as the sternohyoid muscle was exposed. The mice were injected in the tail vein with antibodies to visualize the endothelium (CD31-eFluor450, eBioscience) and leukocytes (CD115-Alexa568, Invitrogen); and with labeled FITC-70kDa dextran (Sigma) to study permeability. MPLSM imaging was performed using a Leica SP5 imaging platform (Leica Microsystems) as previously described ${ }^{16}$. Adhesion was assessed as leukocytes adhering to and staying on the endothelium and transmigration was scored as cells that have transmigrated through the endothelium. Corrections were made for the endothelial surface in $\mathrm{mm}^{2}$, which was calculated by measuring the endothelial diameter and vessel length, and for the measurement time in minutes. Permeability was defined as the ratio between dextran derived extraluminal fluorescence and intraluminal fluorescence and was corrected for vessel volume $\left(\mathrm{mm}^{3}\right)$ and measurement time (minutes).

\section{Transmigration assay}

Flow chambers (iBidi) were coated with human umbilical vein endothelial cells (HUVECs) that were stained for PECAM (mCherry-labeled CD31) and VE-cadherin (55-7H1), stimulated with TNF- $\alpha(10 \mathrm{ng} / \mathrm{mL})$ with or without ADAM10 inhibition by GI254023X (5 $\mu \mathrm{M})$ for $30 \mathrm{~min}$. Neutrophils were isolated from the blood from a healthy volunteer and were injected into the flow system. Differential interference contrast and PECAM-mCherry (594 nm) were recorded using a confocal laser scanning microscope (Zeiss Axiovert). 
Recorded images were scored for adhesion of neutrophils on HUVECs and for transmigration of neutrophils.

\section{ELISAs}

$\mathrm{CX}_{3} \mathrm{CL} 1$ and $\mathrm{CXCL} 16$ ELISA assays (both R\&D systems) were performed on plasma from ADAM $10^{\text {wt }}$ and ADAM $10^{\text {del }}$ mice according to manufacturer's instructions. Analysis was performed using a micro-plate reader (Bio-Rad) at $450 \mathrm{~nm}$.

Plasma Annexin A5 was measured using a commercial enzyme immunoassay (Hyphen BioMed, Neuville-sur-Oise, France) according to manufacturer's instructions. Analysis was performed using a micro-plate reader (Bio-Rad) at $450 \mathrm{~nm}$.

\section{Statistical analysis}

Data are presented as mean \pm the standard error of the mean (SEM). All statistical analyses were performed using the Prism program (GraphPad Software Inc, San Diego, U.S.). Differences between treatment groups were evaluated for statistical significance with two-tailed, unpaired Student's t-test. Significance was accepted at the level of $p<0.05$. ${ }^{*} p<0.05,{ }^{* *} p<0.01$, ${ }^{* * *} p<0.001$. 


\section{Results}

\section{Endothelial ADAM10 plays an important role in adhesion and transmigration.}

We performed a proteomics analysis on supernatant from mouse endothelial BEND5 cells with or without ADAM10 inhibition, to identify the ADAM10 dependent endothelial sheddome, revealing 300 differentially secreted proteins, of which a significant portion (10\%) appeared to be involved in permeability or leukocyte transmigration, including VE-Cadherin, VCAM-1 and PECAM-1. These data suggest a role for ADAM10 in transendothelial migration (TEM) and TEM dependent inflammatory processes such as atherosclerosis. To further investigate this, we measured the effect of ADAM10 inhibition in vitro on transmigration of freshly isolated neutrophils through a HUVEC monolayer. Neutrophil transmigration tended to be reduced after endothelial ADAM10 inhibition (Fig. 1A), while the transmigration time of neutrophils through the layer was increased (Fig. 1B).

A

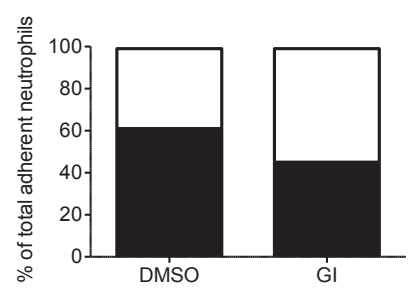

B

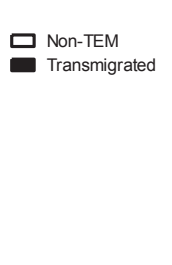

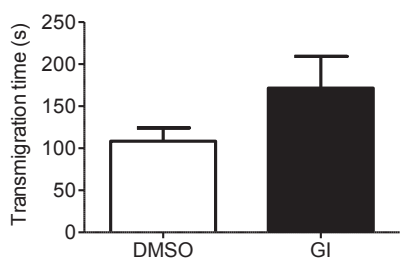

Figure 1. Important role for ADAM10 in leukocyte adhesion and transmigration in vitro.

Transmigrated (black) and non-transmigrated (white) neutrophils, as percentage of total adhered neutrophils $(\mathbf{A})$ and transmigration time $(\mathbf{B})$ in vitro, with or without ADAM10 inhibitor GI254023X.

\section{Deficiency of endothelial ADAM10 leads to significantly increased atherosclerotic lesion size.}

Since endothelial ADAM10 deficiency influences leukocyte adhesion, ADAM10 ${ }^{\text {wt }}$ and ADAM10 del mice were injected with AAV8-PCSK9 to induce atherosclerosis development. Adeno-associated virus will target the liver and induce overexpression of PCSK9, which will degrade LDL receptors (LDLr) 13,14 , rendering these mice hyperlipidemic and prone for atherosclerosis. Directly after injection, mice were placed on WTD for 12 weeks after 
which atherosclerosis development was assessed in the aortic root and brachiocephalic artery. No differences between groups were found in body weight $\left(26.3 \pm 0.8 \mathrm{~g}\right.$ for ADAM10 ${ }^{\text {wt }}$ vs $27.9 \pm 0.7 \mathrm{~g}$ for ADAM10 del mice, data not shown), vessel morphology (Suppl. Fig. 1A) or liver inflammation (Suppl. Fig. 1B-C). Furthermore, plasma cholesterol and triglyceride levels on chow and after WTD feeding were not different between ADAM $10^{\text {del }}$ and ADAM10 ${ }^{\text {wt }}$ mice (Fig. 2A-B). Next, we analyzed the pattern of circulating leukocyte subsets in ADAM10 $10^{\text {del }}$ and ADAM10 $10^{\text {wt }}$ mice by flow cytometry at 0,6 and 12 weeks of WTD feeding. Apart from a minor increase in total $\mathrm{CD}^{+} \mathrm{T}$-cell numbers at week 12 , no significant differences in number of total CD45 leukocytes or myeloid lineages between genotypes were found for all time points (Fig. 2C-D and Suppl. Fig. 1D).

A

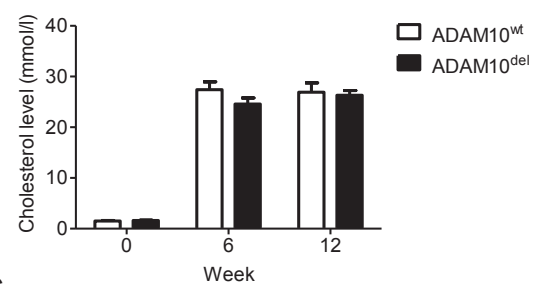

B

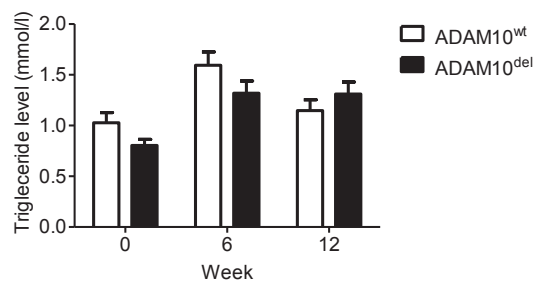

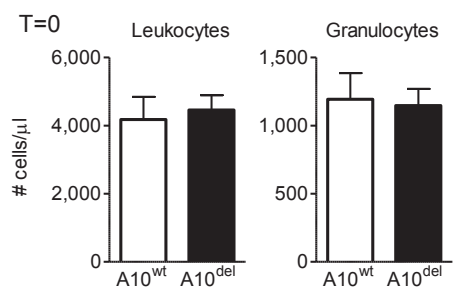
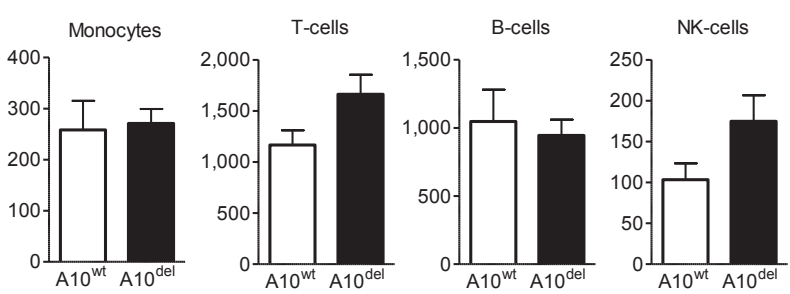

$\mathrm{D}$
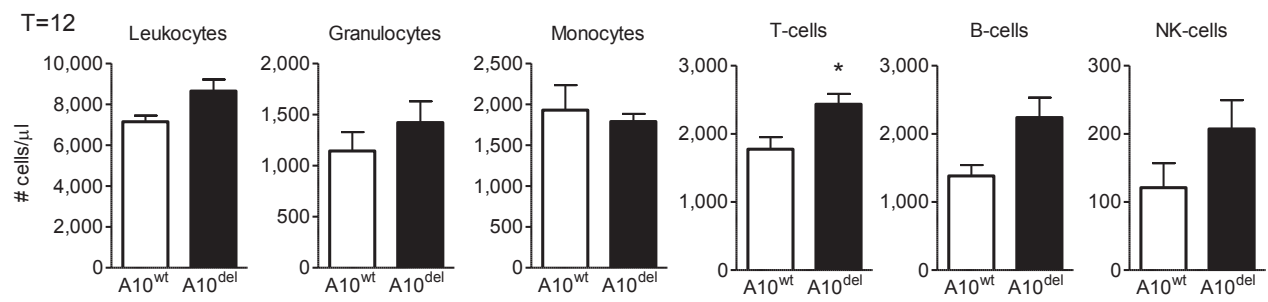

Figure 2. ADAM10 deficiency does only effect T-cell levels after WTD feeding. Plasma cholesterol (A) and triglyceride (B) levels of ADAM10 wt and ADAM10 del mice. FACS analysis showing absolute numbers of leukocytes, granulocytes, monocytes, T-cells, B-cells and NK-cells in blood of ADAM10 ${ }^{\text {wt }}$ and ADAM10 del mice at $t=0$ weeks (C) or $t=12$ weeks (D) of Western-type diet feeding. 
Deficiency of endothelial ADAM10 revealed $45 \%$ increased lesion size in the aortic roots (Fig. 3A-C). Additionally, lesions from ADAM10 del mice appeared to be more advanced compared to lesions from ADAM10 ${ }^{\text {wt }}$ mice (Fig. 3D). Besides the aortic roots, lesion area in the brachiocephalic artery was also markedly increased in endothelial ADAM10 deficient compared to wildtype mice (Suppl. Fig. 2A-B). Moreover, significantly more plaque hemorrhage was observed in ADAM10 ${ }^{\text {del }}$ mice compared to ADAM10 $10^{\text {wt }}$ mice (Fibrin positivity score of $1.75 \pm 0.60$ versus $0.25 \pm 0.16$ respectively, $p=0.03$ ). Atherosclerotic lesions were further examined by routine pathological examination for their composition, i.e. collagen content, necrosis, foam cell content or amount and location of inflammatory cells. Lesions from ADAM10 del mice showed significantly more plaque-necrosis and less foam cells (Fig. 3E). Further histological analysis confirmed the augmented necrotic core content of atherosclerotic lesions from ADAM10 ${ }^{\text {del }}$ mice (Fig. 3F).

Additionally, the decreased amount of foam cells/macrophages observed by routine pathological examination was quantified using MAC3 staining. Lesions from ADAM10 del mice had a $51 \%$ decrease in the percentage of $\mathrm{MAC}^{+}$macrophage content, both in moderate and advanced lesions (Fig. 4A-D). However, no difference was observed in absolute plaque macrophage content, suggesting that the decreased macrophage content merely reflected plaque progression, i.e. increased necrosis (Suppl. Fig. 3A-B). Plaque $\mathrm{Ly}_{6 \mathrm{G}}{ }^{+}$granulocyte content did not differ between the genotypes (Fig. 4E-H), while likewise, no differences were found in relative plaque collagen content as quantified by Sirius Red staining (Fig. 4I-L). Differentiating on the basis of collagen structure (loosely patched, immature, thin collagen (green) versus tightly packed, mature, thick collagen fibers (red)) by using a polarization filter, we were able to demonstrate that this increase is equally present in all collagen types, suggesting no changes in collagen maturation (Fig. 4M-O). 
A
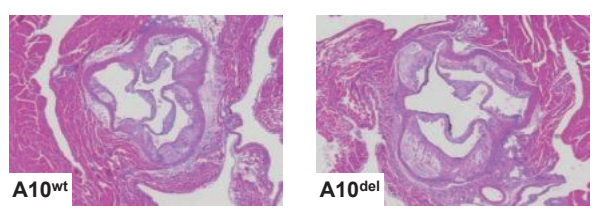

D

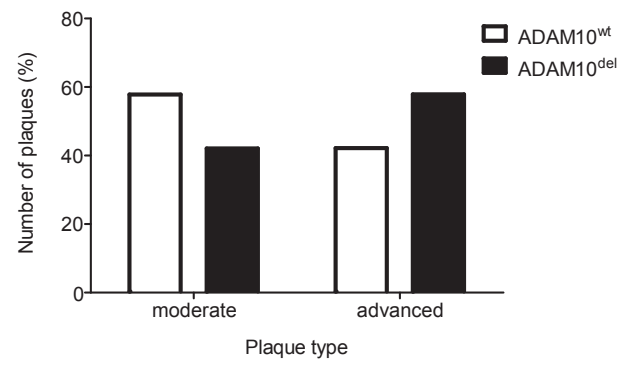

$\mathrm{F}$

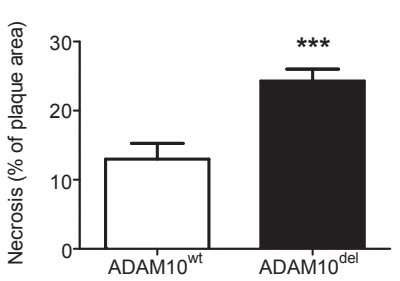

C

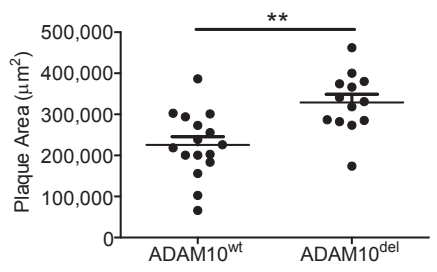

$\mathrm{E}$

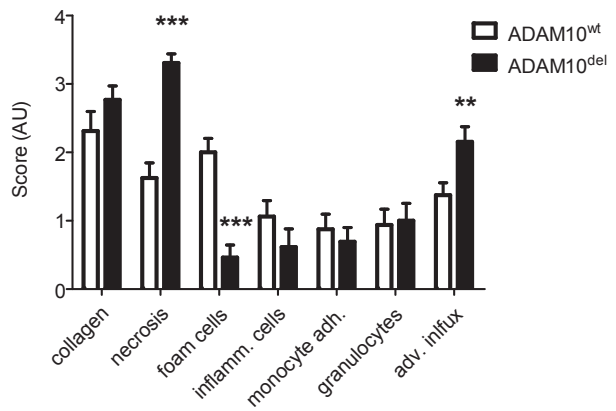

Figure 3. Endothelial ADAM10 deficiency results in larger and more necrotic atherosclerotic lesions. Representative pictures of Hematoxylin-Eosin-stained sections of the aortic root of ADAM10 wt $(A)$ and $\operatorname{ADAM10}$ del (B) mice, magnification 40x. Quantification of plaque area (C), plaque classification (D) and routine pathological examination of plaque composition (E). Quantification of percentage necrosis of plaque area in ADAM10 wt and ADAM10 $10^{\text {del }}$ mice $(\mathbf{F})$. 
A

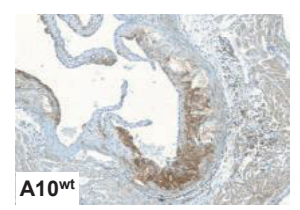

E
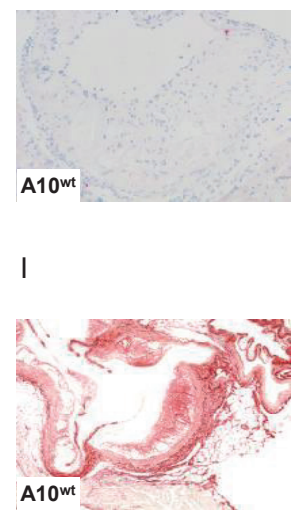

M

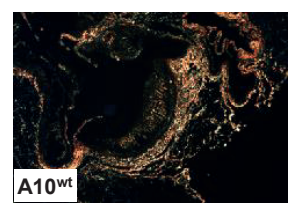

B

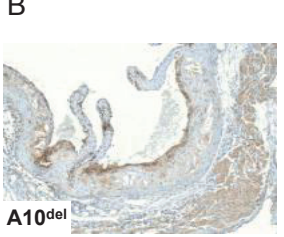

$\mathrm{F}$

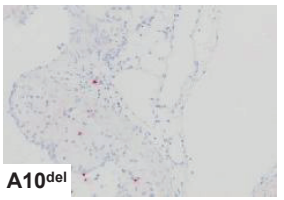

$J$

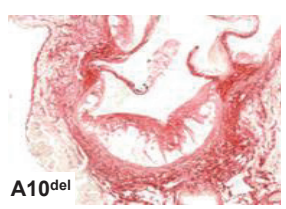

N

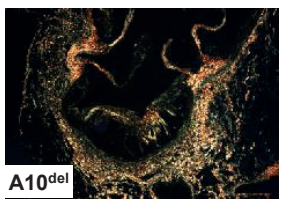

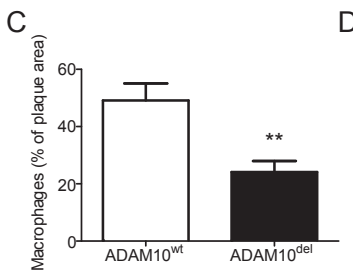

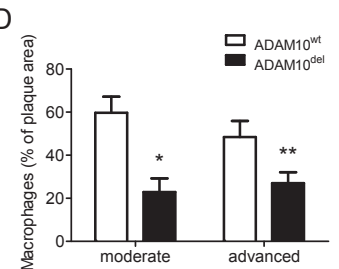

G

$\mathrm{H}$
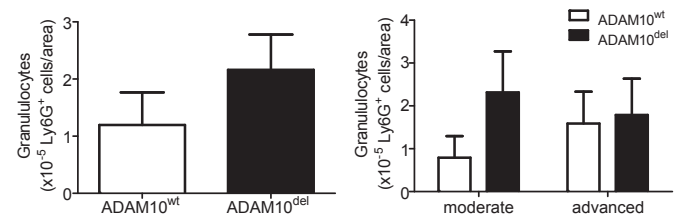

K

$\mathrm{L}$
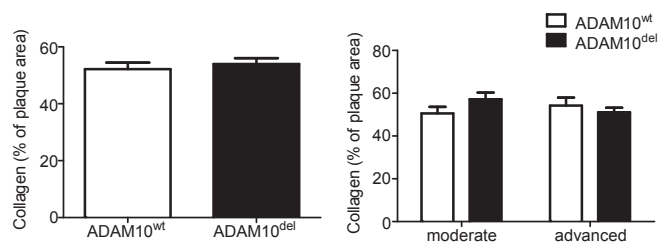

$\mathrm{O}$

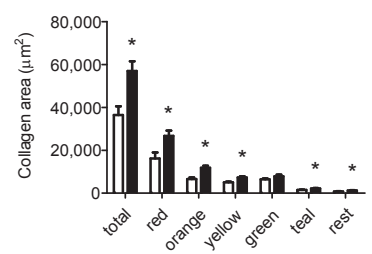

Figure 4. ADAM10 deficiency results in relative reduced plaque macrophage content. Representative pictures and quantification of $\mathrm{MAC3}^{+}$macrophages (A-D), Ly6G+ granulocytes(E-H), Sirus red stained collagen by normal light microscopy representing total collagen (I-L) and by polarizing light microscopy, representing different collagen fibers ranging from thick, mature collagen (red) to loosely packed, thin collagen fibers (green) (M-O) of ADAM10 ${ }^{\text {wt }}$ and ADAM10 ${ }^{\text {del }}$ mice, magnification 40x. 


\section{Deficiency of endothelial ADAM10 significantly reduces plasma Annexin A5 levels and seem to increase adhesion and transmigration in vivo.}

The increased atherosclerosis might be explained by increased leukocyte attraction via chemokines. Therefore, we next determined the effect of endothelial ADAM10 deficiency on two well known substrates of ADAM10, the chemokines $\mathrm{CX}_{3} \mathrm{CL} 1$ and $\mathrm{CXCL} 16$. These chemokines act as adhesion molecules in membrane bound state to acquire chemotactic functions, after shedding by ADAM10, in soluble state. However, we could not observe any differences in soluble chemokine levels in plasma of ADAM10 del and ADAM10 ${ }^{\text {wt }}$ mice (Fig. 5A-B), suggesting they are, at least in soluble state, not critical for the increased atherosclerosis development observed in ADAM10 $10^{\text {del }}$ mice. Interestingly, our proteomics analysis identified Annexin A5 as possible ADAM10 target. A well-known function of Annexin A5 is its binding to phosphatidylserine, a surface marker for stressed or dying cells ${ }^{17}$. Recently, Annexin A5 treatment was reported to reduce leukocyte adhesion and transmigration in vitro and plaque macrophage content in vivo, indicating a role for Annexin A5 in leukocyte recruitment and atherosclerosis development 18. Confirming our proteomics data, in vitro inhibition of endothelial ADAM10 led to significantly reduced Annexin A5 levels in endothelial cell medium (Fig. 5C). In line with this, atherogenic ADAM10 del mice displayed significantly reduced (54\%) plasma levels of Annexin A5 (Fig. 5D). In vivo imaging of microvessels in the sternohyoid muscle of ADAM $10^{\text {wt }}$ versus ADAM10 del mice (12 weeks WTD feeding) revealed more permeable vessels with a higher degree of leukocyte adhesion and transmigration in ADAM10 del compared to ADAM10 ${ }^{\text {wt }}$ mice (Fig. 6A-C). In conclusion, ADAM10 deficiency remarkably increases atherosclerotic lesion size and progression, possibly by modulating leukocyte recruitment. 
A

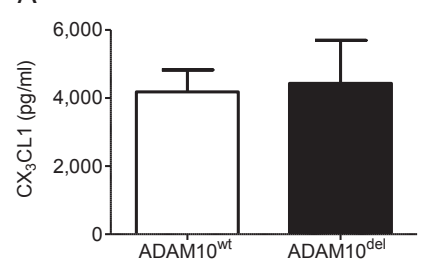

C

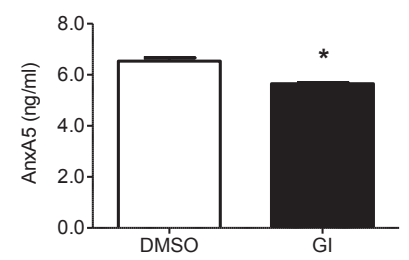

B

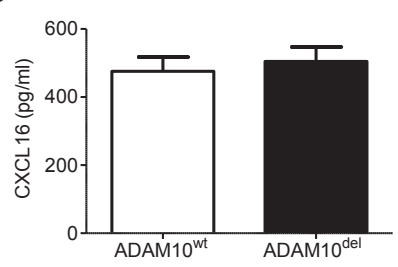

D

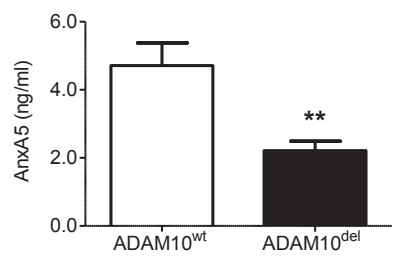

Figure 5. Annexin A5 plasma levels are significantly reduced in ADAM10 deficient mice. Soluble $\mathrm{CX}_{3} \mathrm{CL} 1$ (A), CXCL16 (B) and Annexin A5 (C) in plasma of ADAM10 ${ }^{\text {wt }}$ and ADAM10 del mice after 12 weeks Western-type diet feeding. Annexin A5 concentration in conditioned medium from BEND5 cells incubated with or without GI254023X for 24 hrs (D).

A

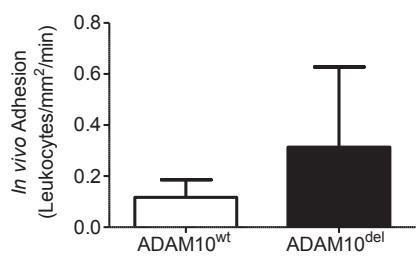

B

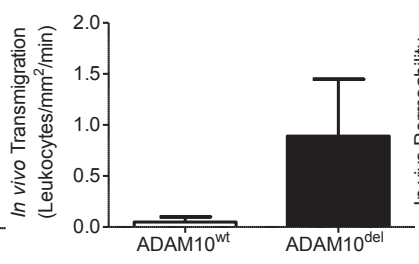

C

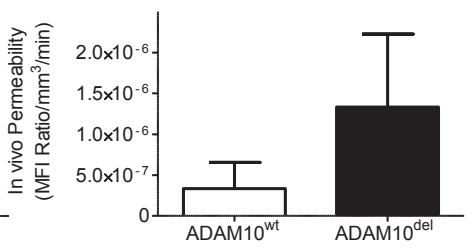

Figure 6. In vivo ADAM10 deficiency increases leukocyte adhesion and transmigration. Adhesion (A), transmigration (B) and permeability (C) in ADAM10 $10^{\text {wt }}$ and ADAM10 del mice in vivo. Adhesion and transmigration data are corrected for endothelial surface area in $\mathrm{mm}^{2}$ and time in minutes. For permeability data the mean fluorescent intensity (MFI) is corrected for the vessel volume in $\mathrm{mm}^{3}$ and time in minutes. 


\section{Discussion}

In this study we determined the effect of endothelial ADAM10 deficiency on atherosclerosis development, after induction of sustained LDLr knockdown/hyperlipidemia by AAV8-PCSK9 gene transfer ${ }^{13}$. The approach of atherosclerosis induction was successful, based on markedly increased plasma cholesterol levels and atherosclerosis development. AlthoughADAM10 is involved in the shedding of various adhesion and junctional molecules, we surprisingly observed that ADAM $10^{\text {del }}$ mice have significantly increased lesion size and progression compared to ADAM10 wt mice. In accordance with this increased atherosclerotic lesion size, we observed a trend towards increased leukocyte adhesion and transmigration to the sternohyoid muscle capillary endothelium in ADAM10 del mice. In keeping, ADAM10 deficient mice had increased vessel permeability, which at least partly could explain the increased leukocyte transmigration, albeit it that this effect did not reach statistical significance due to too small group sizes. In sharp contrast, in vitro we observed reduced transmigration, with increased transmigration time after pharmacological ADAM10 inhibition, which is in line with findings by Schulz et al. showing an important role of ADAM10 in endothelial permeability and T-cell transmigration ${ }^{12}$. These paradoxical findings could be explained by differences in the duration of ADAM10 (functional) knockdown and the method of knockdown, comparing pharmacological inhibition versus genetic ablation. Furthermore, differences in experimental set-up may underlie this paradox, as the in vivo studies were conducted in mice, and the in vitro setup under laminar flow was based on human cells. Additionally, the in vitro setup may reflect conditions of acute inflammation, whereas the in vivo setup studies the impact of ADAM10 deficiency in WTD induced chronic inflammation. Therefore, the chronic inflammatory situation could be the consequence of endothelial damage, induced by the accumulation of adhered leukocytes. A study using retinal endothelium already showed that sustained adhesion of leukocytes can cause damage ${ }^{19}$. Eventually, when the endothelium is damaged it will get leaky, which might explain the observed increase in permeability and transmigration.

While endothelial ADAM10 deficiency did not alter major leukocyte subsets relevant to atherosclerosis, such as monocytes and neutrophils, it was associated with a significant increase in $\mathrm{CD}^{+} \mathrm{T}$-cells, and especially in $\mathrm{CD} 8^{+}$ cytotoxic T-cells in blood (data not shown). Further research is needed to 
determine how endothelial ADAM10 deficiency can increase circulating T-cell levels, since transendothelial migration is also important for the recruitment of lymphocytes from lymphoid organs to the periphery ${ }^{20}$. T-cells are known to contribute to the pathogenesis of atherosclerosis. This was primarily attributed to $\mathrm{CD}^{+} \mathrm{T}$-cells ${ }^{4}$, although a recent study stressed that the importance of $\mathrm{CD}^{+} \mathrm{T}$-cells in atherogenesis should not be underestimated, since depletion of $\mathrm{CD}^{+}{ }^{+}$T-cells ameliorated atherogenesis ${ }^{21}$. Quantitation of T-cell presence in the atherosclerotic lesions, could give further insight into the effects of ADAM10 deficiency on $\mathrm{CD}^{+} \mathrm{T}$-cell influx into the lesion and their impact on atherogenesis.

Endothelial ADAM10 deficiency did not seem to significantly influence the shedding of well-known substrates such as $\mathrm{CX}_{3} \mathrm{CL} 1$ and CXCL16, in that plasma levels of soluble form of both chemokines were not different between the genotypes. This does not necessarily imply that the expression of membrane bound chemokines is equivalent as well, since upregulation of their expression and membrane presentation can at this point not be excluded. Besides these well-known ADAM substrates, also other substrates that have not yet been investigated in this study, such as the junctional molecules VECadherin and junctional adhesion molecules (JAMs), could be implicated in the ADAM10 deficiency associated increase in atherosclerosis.

Another potential mechanism that could be involved is the receptor for advanced glycation end products (RAGE) since it also has been described as a substrate for ADAM10 and is prominently expressed on activated endothelial cells, where it mediates leukocyte adhesion and transmigration ${ }^{22}$, ${ }^{23}$. Especially since AGEs, the ligands for RAGE that are abundantly present in atherosclerotic conditions, have also been shown to increase leukocyte adhesion in a RAGE specific manner ${ }^{24}$. Moreover, Harja et al. showed that RAGE plays a crucial role in atherosclerosis development by augmenting endothelial dysfunction and VCAM-1 expression in $\mathrm{ApoE}^{-/-}$mice ${ }^{25}$.

Interestingly, we did observe marked effects on Annexin A5 levels in the plasma. Annexin A5 is known for its binding to phosphatidylserine, expressed on stressed and apoptotic cells. Furthermore, Annexin A5 has been shown to exert anti-inflammatory properties via this phosphatidylserine binding, which may shield the cells from inflammatory cell contact ${ }^{17,26}$. Recently, shortterm intraperitoneal Annexin A5 treatment was reported to reduce plaque macrophage content, an effect that was attributed to diminished leukocyte 
adhesion and transmigration ${ }^{18}$. Therefore it is conceivable that the observed reduced plasma Annexin A5 levels in ADAM10 del mice, are involved in the increased lesion size and progression upon ADAM10 deficiency. To date, it is not known how ADAM10 modulates circulating/extracellular Annexin A5 levels. Recently, Annexin A1 has been shown to be proteolytically cleaved by ADAM10, thereby creating a peptide with monocyte chemotactic activity ${ }^{27}$. Further research is necessary to elucidate whether ADAM10 can also cleave Annexin A5, or whether ADAM10 modulates AnxA5 secretion or even binding to the cells. Furthermore, it remains to be determined whether Annexin A5 is indeed causally involved in the augmented atherosclerosis development in endothelial ADAM10 deficient mice.

In conclusion, this study surprisingly shows that endothelial ADAM10 protects against atherosclerosis, by modulating leukocyte recruitment. The precise mechanisms behind this effect remain to be elucidated. 


\section{Acknowledgements}

This study was supported by Dr. E. Dekker post-doctoral grant nrs 2012T079 from the Netherlands Heart Foundation (to Marjo M.P.C. Donners) and the Scholarship of the Professors' fund / Limburg University Fund 2014 (to Marjo M.P.C. Donners and Emiel P.C. van der Vorst). 


\section{References}

1. Hansson GK, Hermansson A. The immune system in atherosclerosis. Nature immunology. 2011;12:204-212

2. Gijbels MJ, van der Cammen M, van der Laan LJ, Emeis JJ, Havekes LM, Hofker $\mathrm{MH}$, et al. Progression and regression of atherosclerosis in apoe3-leiden transgenic mice: An immunohistochemical study. Atherosclerosis. 1999;143:1525

3. Ross R. Atherosclerosis--an inflammatory disease. The New England journal of medicine. 1999;340:115-126

4. Weber $\mathrm{C}$, Noels $\mathrm{H}$. Atherosclerosis: Current pathogenesis and therapeutic options. Nature medicine. 2011;17:1410-1422

5. Campbell JJ, Hedrick J, Zlotnik A, Siani MA, Thompson DA, Butcher EC. Chemokines and the arrest of lymphocytes rolling under flow conditions. Science. 1998;279:381-384

6. Liu Y, Shaw SK, Ma S, Yang L, Luscinskas FW, Parkos CA. Regulation of leukocyte transmigration: Cell surface interactions and signaling events. Journal of immunology. 2004;172:7-13

7. Dreymueller D, Pruessmeyer J, Groth E, Ludwig A. The role of adam-mediated shedding in vascular biology. European journal of cell biology. 2012;91:472-485

8. van der Vorst EP, Keijbeck AA, de Winther MP, Donners MM. A disintegrin and metalloproteases: Molecular scissors in angiogenesis, inflammation and atherosclerosis. Atherosclerosis. 2012;224:302-308

9. Donners MM, Wolfs IM, Olieslagers S, Mohammadi-Motahhari Z, Tchaikovski V, Heeneman $S$, et al. A disintegrin and metalloprotease 10 is a novel mediator of vascular endothelial growth factor-induced endothelial cell function in angiogenesis and is associated with atherosclerosis. Arteriosclerosis, thrombosis, and vascular biology. 2010;30:2188-2195

10. Li X, Yan Y, Huang W, Yang Y. The study of the inhibition of the recombinant tace prodomain to endotoxemia in mice. International journal of molecular sciences. 2009;10:5442-5454

11. Ludwig A, Weber C. Transmembrane chemokines: Versatile 'special agents' in vascular inflammation. Thrombosis and haemostasis. 2007;97:694-703

12. Schulz B, Pruessmeyer J, Maretzky T, Ludwig A, Blobel CP, Saftig P, et al. Adam10 regulates endothelial permeability and t-cell transmigration by proteolysis of vascular endothelial cadherin. Circulation research. 2008;102:1192-1201

13. Bjorklund MM, Hollensen AK, Hagensen MK, Dagnaes-Hansen F, Christoffersen C, Mikkelsen JG, et al. Induction of atherosclerosis in mice and hamsters without 
germline genetic engineering. Circulation research. 2014;114:1684-1689

14. Maxwell KN, Fisher EA, Breslow JL. Overexpression of pcsk9 accelerates the degradation of the IdIr in a post-endoplasmic reticulum compartment. Proceedings of the National Academy of Sciences of the United States of America. 2005;102:2069-2074

15. MacKenna DA, Omens JH, Covell JW. Left ventricular perimysial collagen fibers uncoil rather than stretch during diastolic filling. Basic research in cardiology. 1996;91:111-122

16. Rademakers T, Douma K, Hackeng TM, Post MJ, Sluimer JC, Daemen MJ, et al. Plaque-associated vasa vasorum in aged apolipoprotein e-deficient mice exhibit proatherogenic functional features in vivo. Arteriosclerosis, thrombosis, and vascular biology. 2013;33:249-256

17. Schutters K, Reutelingsperger C. Phosphatidylserine targeting for diagnosis and treatment of human diseases. Apoptosis : an international journal on programmed cell death. 2010;15:1072-1082

18. Burgmaier M, Schutters K, Willems B, van der Vorst EP, Kusters D, Chatrou M, et al. Anxa5 reduces plaque inflammation of advanced atherosclerotic lesions in apoe(-/-) mice. Journal of cellular and molecular medicine. 2014;18:2117-2124

19. Joussen AM, Murata T, Tsujikawa A, Kirchhof B, Bursell SE, Adamis AP. Leukocytemediated endothelial cell injury and death in the diabetic retina. The American journal of pathology. 2001;158:147-152

20. Shulman Z, Cohen SJ, Roediger B, Kalchenko V, Jain R, Grabovsky V, et al. Transendothelial migration of lymphocytes mediated by intraendothelial vesicle stores rather than by extracellular chemokine depots. Nature immunology. 2012;13:67-76

21. Kyaw T, Winship A, Tay C, Kanellakis P, Hosseini H, Cao A, et al. Cytotoxic and proinflammatory cd8+ $t$ lymphocytes promote development of vulnerable atherosclerotic plaques in apoe-deficient mice. Circulation. 2013;127:1028-1039

22. Kierdorf K, Fritz G. Rage regulation and signaling in inflammation and beyond. Journal of leukocyte biology. 2013;94:55-68

23. Raucci A, Cugusi S, Antonelli A, Barabino SM, Monti L, Bierhaus A, et al. A soluble form of the receptor for advanced glycation endproducts (rage) is produced by proteolytic cleavage of the membrane-bound form by the sheddase a disintegrin and metalloprotease 10 (adam10). FASEB journal : official publication of the Federation of American Societies for Experimental Biology. 2008;22:37163727

24. Basta G, Lazzerini G, Massaro M, Simoncini T, Tanganelli P, Fu C, et al. Advanced glycation end products activate endothelium through signal-transduction receptor rage: A mechanism for amplification of inflammatory responses. 
Circulation. 2002;105:816-822

25. Harja E, Bu DX, Hudson BI, Chang JS, Shen X, Hallam K, et al. Vascular and inflammatory stresses mediate atherosclerosis via rage and its ligands in apoe-I- mice. The Journal of clinical investigation. 2008;118:183-194

26. van Genderen HO, Kenis H, Hofstra L, Narula J, Reutelingsperger CP. Extracellular annexin a5: Functions of phosphatidylserine-binding and two-dimensional crystallization. Biochimica et biophysica acta. 2008;1783:953-963

27. Blume KE, Soeroes S, Keppeler H, Stevanovic S, Kretschmer D, Rautenberg M, et al. Cleavage of annexin a1 by adam10 during secondary necrosis generates a monocytic "find-me" signal. Journal of immunology. 2012;188:135-145 


\section{Supplemental Figures}

A

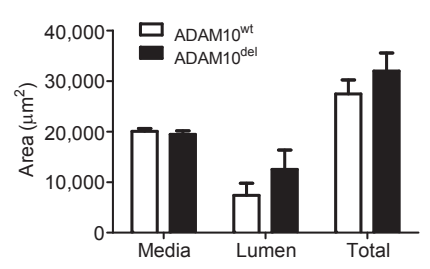

$\mathrm{D}$
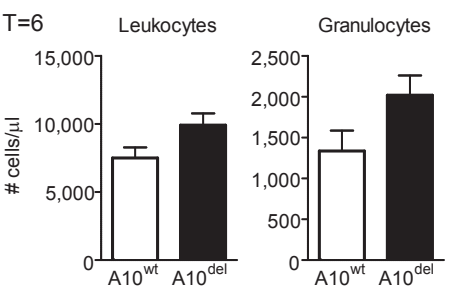

$B$

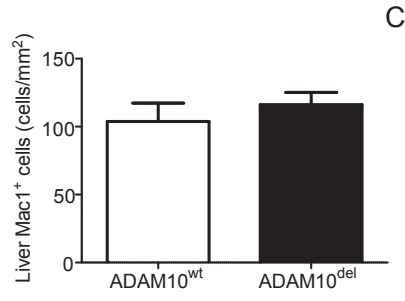

C

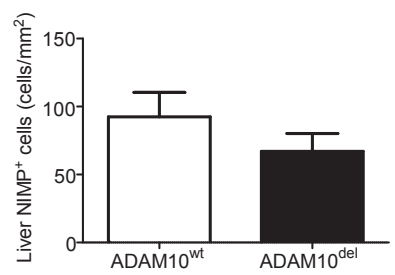

Supplemental Figure 1. No systemic differences between ADAM10 ${ }^{\text {wt }}$ and ADAM10 del mice. Morphometric analysis of carotid vessels of $A D A M 10^{\text {wt }}$ and ADAM10 del mice $(\mathbf{A})$. Liver inflammation analysis showing cell counts of MAC1+ macrophages $(B)$ and NIMP1+ granulocytes $(\mathbf{C})$ in livers of ADAM $10^{\text {wt }}$ and ADAM $10^{\text {del }}$ mice. FACS analysis showing absolute numbers of leukocytes, granulocytes, monocytes, T-cells, B-cells and NK-cells in blood of ADAM $10^{\text {wt }}$ and ADAM10 $10^{\text {del }}$ mice at $t=6$ weeks of Western-type diet feeding (D).

A

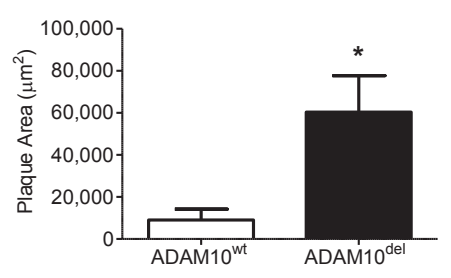

B

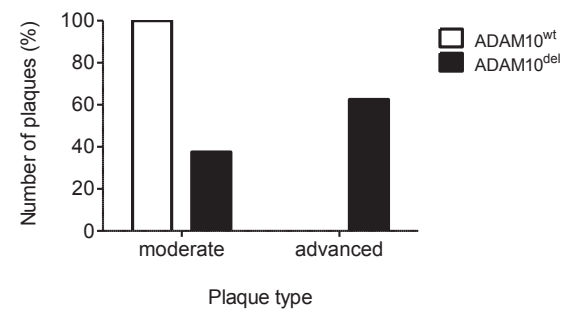

Supplemental Figure 2. Endothelial ADAM10 deficiency increases lesion size and progression in the brachiocephalic artery. Quantification of plaque area $(\mathbf{A})$ and plaque classification (B) in brachiocephalic artery of ADAM10 $10^{\text {wt }}$ and ADAM10 del mice. 
A

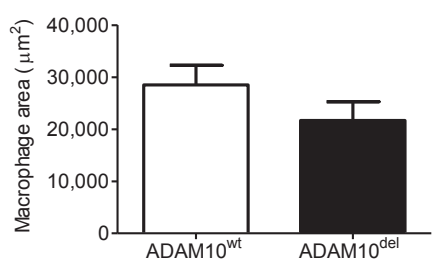

$\mathrm{B}$

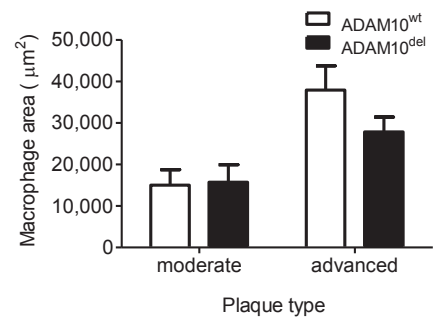

Supplemental Figure 3. Total macrophage area is not different between both genotypes. Quantification of total $\mathrm{MAC3}^{+}$macrophage content of atherosclerotic lesion of ADAM $10^{\text {wt }}$ and ADAM10 del mice (A-B). 


\section{Chapter 6}

\section{High Density Lipoproteins suppress chemokine expression and proliferation in human vascular smooth muscle cells}

Emiel P.C. van der Vorst", Laura Z. Vanags*, Louise L. Dunn, Hamish C. Prosser, Kerry-Anne Rye and Christina A. Bursill

*Authors contributed equally

FASEB J., 2013 Apr;27(4):1413-25 


\section{Abstract}

The inflammatory chemokines CCL2, CCL5 and CX ${ }_{3} \mathrm{CL} 1$ stimulate vascular smooth muscle cell (SMC) proliferation. High-density lipoproteins (HDL) exhibit potent cardioprotective and anti-inflammatory properties. We therefore sought to determine the effect of reconstituted HDL (rHDL) on SMC chemokine expression and proliferation and elucidate the mechanisms. Preincubation of primary human SMCs with rHDL containing apolipoprotein (apo) A-I and phosphatidylcholine $(600 \mu \mathrm{g} / \mathrm{ml}$, final apoA-I concentration), prior to stimulation with TNF- $\alpha$, inhibited CCL2, CCL5 and $\mathrm{CX}_{3} \mathrm{CL} 1$ protein levels. The chemokine receptors CCR2 and $\mathrm{CX}_{3} \mathrm{CR} 1$ were also reduced by $\mathrm{rHDL}$. Incubation with $\mathrm{rHDL}$ reduced the NF-KB subunit, p65, in the nucleus and phosphorylated $\mathrm{I} K \mathrm{~B} \alpha$, both regulators of chemokine expression. Furthermore, rHDL inhibited the upstream signaling proteins phosphoinositide 3-kinase and phosphorylated Akt (pAkt). Incubation with rHDL strikingly suppressed SMC proliferation and ERK phosphorylation (pERK). Finally, siRNA knockdown of the scavenger receptor SR-B1 attenuated $\mathrm{HHDL}$-induced inhibition of SMC chemokine expression, p65 and proliferation, indicating that SR-B1 plays a key role in mediating these effects. Thus, rHDL reduces SMC chemokine expression (via NF-kB/pAkt inhibition) and proliferation (via pERK inhibition). This has important implications for atherosclerosis and preventing the pathogenesis of neointimal hyperplasia, the main cause of early vein graft/ stent failure. 


\section{Introduction}

The protective properties of high-density lipoproteins (HDL) on the vasculature are well established ${ }^{1}$. Although this has been primarily attributed to the ability of HDL to efflux cholesterol from cells in the periphery, especially macrophages in the arterial wall, HDL have also been shown to exhibit antithrombotic, anti-oxidative and in particular anti-inflammatory properties ${ }^{2}$.

Chemokines are small proteins that direct cellular trafficking and thus play an important role in inflammatory processes ${ }^{3}$. They are divided into four classes $\left(\mathrm{C}, \mathrm{CC}, \mathrm{CXC}, \mathrm{CX}_{3} \mathrm{C}\right)$, based on the number and location of conserved cysteine residues. The chemokines CCL2 (MCP-1) and CCL5 (RANTES) are members of the CC-chemokine class and $\mathrm{CX}_{3} \mathrm{CL} 1$ (fractalkine) is the only known member of the $\mathrm{CX}_{3} \mathrm{C}$ class. Chemokines have a well-documented role in directing monocyte recruitment and migration. More recently they have been implicated in the direct promotion of SMC proliferation and SMC neointimal expansion. For example, in vitro studies show that incubation with the chemokines CCL2, CCL5 or $\mathrm{CX}_{3} \mathrm{CL} 1$ causes significant increases in SMC proliferation ${ }^{4-8}$. Neointimal hyperplasia is also attenuated in murine arterial injury models that have targeted chemokine deletions (eg CCL5 ${ }^{5}$ ) and CCL2 is rapidly elevated following vascular injury ${ }^{9-11}$. Chemokines mediate these effects via cognate chemokine receptors. For example, knockout mice with deletions in the chemokine receptors CCR5 ${ }^{12}$ (receptor to CCL5) and CCR2 ${ }^{13}$ (receptor to CCL2) have reduced neointimal hyperplasia following vascular injury. Furthermore, treatment of SMCs with a competitive antagonist of CCR2 ${ }^{14}$, or short hairpin RNA targeted against CCR2, inhibit SMC proliferation in in vivo models ${ }^{13,15}$. Studies in vitro have also shown that an antagonist of $\mathrm{CX}_{3} \mathrm{CR} 1$ (receptor to $\mathrm{CX}_{3} \mathrm{CL} 1$ ) abrogates the proliferative effects of $\mathrm{CX}_{3} \mathrm{CL} 1$ in SMCs ${ }^{8}$. Excessive neointimal hyperplasia causes restenosis, which can limit the success of percutaneous coronary interventions (PCI) such as balloon angioplasty and stent implantation, which are commen interventions for atherosclerosis patients ${ }^{16}$. This highlights the potential significance of chemokine-induced SMC proliferation and suggests that an agent capable of suppressing vascular SMC inflammation, chemokine expression and proliferation would be potentially valuable in the context of PCls. 
HDL have potent anti-inflammatory properties ${ }^{2}$. We and others have shown that reconstituted $\mathrm{HDL}(\mathrm{rHDL})$ suppress the expression of chemokines including: CCL2, CCL5 and $\mathrm{CX}_{3} \mathrm{CL} 1$ in in vitro studies in various celltypes (e.g. endothelial cells, monocytes) and in vivo studies in rabbits ${ }^{17-21}$. However, the effect of HDL on SMC chemokine expression and proliferation is currently unexplored.

We therefore sought to determine whether HDL can inhibit SMC chemokine and chemokine receptor expression and proliferation. In this study we report that rHDL are able to inhibit the expression of the chemokines CCL2, CCL5 and $\mathrm{CX}_{3} \mathrm{CL} 1$ via suppression of PI3K/Akt and NF-KB signaling pathways. We also report that $\mathrm{rHDL}$ strikingly inhibit SMC proliferation, via ERK inhibition and that $\mathrm{rHDL}$ suppress the chemokine receptors CCR2 and $\mathrm{CX}_{3} \mathrm{CR} 1$. siRNA knockdown experiments indicated that these inhibitory effects of rHDL involved the scavenger receptor SR-B1.

This study provides mechanistic insight into a unique role for rHDL, whereby rHDL may protect against the development of restenosis and early vein graft/ stent failure in atherosclerosis patients. 


\section{Methods}

\section{Preparation of HDL, apoA-I, discoidal reconstituted HDL and phospholipid vesicles}

Expired, autologously donated plasma samples were used for the study. Approval for subsequent use was obtained at the time of collection. The native HDL (nHDL) fraction (1.063-1.21 $\mathrm{g} / \mathrm{ml}$ ) was isolated from pooled samples of normal human plasma ( 5 donors) by ultracentrifugation then dialysed against phosphate buffered saline (PBS) ${ }^{22}$. For the isolation of apoA-I, nHDL were delipidated and subjected to anion-exchange chromatography using a fast protein liquid chromatography (FPLC) system ${ }^{22}$. Discoidal rHDL containing apoA-I complexed to 1-palmitoyl-2-linoleoyl-phosphatidylcholine (PLPC, Avanti Polar Lipids, Alabaster, AL, USA), initial PLPC/apoA-I molar ratio 100:1, were prepared by the cholate dialysis method ${ }^{23}$. Following dialysis, the final PLPC/apoA-I ratio was $~ 80: 1$. Phospholipid vesicles (PLPC) were prepared by sonication ( $3 \times 5$ min on ice) in PBS and cholate, followed by dialysis against PBS. The protein concentration of apoA-I was determined using the bicinchoninic acid (BCA) assay (Thermo Scientific, Scoresby, Victoria, Australia). Phospholipid concentrations were enzymatically determined (Wako, Richmond, VA, USA) ${ }^{24}$.

\section{Cell culture and incubations}

Primary human umbilical vein smooth muscle cells (HUVSMCs, Cell Applications, San Diego, CA, USA) were cultured in smooth muscle cell growth medium (Cell Applications) and grown until $80 \%$ confluent. They were then incubated with rHDL, apoA-I, nHDL (all $600 \mu \mathrm{g} / \mathrm{ml}$, final apoA-I concentration), phospholipid vesicles $(2 \mu \mathrm{M})$, cyclodextrin ( $54 \mu \mathrm{M}{ }^{25}$, Sigma-Aldrich, Sydney, Australia) or PBS (Control) for 16 hrs. Following incubation, the cells were washed twice with PBS and stimulated with TNF- $\alpha$ (final concentration $1 \mathrm{ng} /$ $\mathrm{ml}$ ) (R\&D Systems, Minneapolis, MN, USA) for $4 \mathrm{hrs}$. For signaling studies (PI3K, pAkt and pERK), HUVSMCs were stimulated for 10 min with TNF- $\alpha$ (final concentration $1 \mathrm{ng} / \mathrm{ml}$ ), washed with PBS, then harvested using a cell scraper. All experiments were performed in quadruplicate $(n=4)$ and repeated three-five times. 


\section{Real-time PCR}

RNA extraction was performed using Total RNA Extraction Kit (Qiagen, Victoria, Australia), according to manufacturer's instructions, after which RNA content was quantified using the Nanovue (General Electric, Rydalmere, NSW, Australia). Total RNAwas normalized and reverse-transcribed in triplicate using iScript (Bio-Rad, Gladesville, NSW, Australia). Real-time PCR was conducted with SybrGreen (Bio-Rad) using primer sequences (Geneworks, Hindmarsh, SA, Australia) for CCL2 forward: 5'-TCATAGCAGCCACCTTCATT-3' CCL2 reverse: 5'-TCGGAGTTTGGGTTTGCTT-3'

CCL5 forward: 5'-TCCTCATTGCTACTGCCCT-3'

CCL5 reverse: 5'-TTGGCGGTTCTTTCGGGTGA-3'

CX ${ }_{3}$ CL1 forward: 5'-TATCTCTGTCGTGGCTGCT-3'

$\mathrm{CX}_{3} \mathrm{CL} 1$ reverse: 5'-TCCTTGACCCATTGCTCCTT-3'. Relative mRNA quantification was calculated with the $2^{-(\triangle C T)}$ method and normalised to GAPDH.

\section{Enzyme-Linked Immunosorbent Assay (ELISA)}

Harvested HUVSMCs were homogenised in cell lysis buffer $(80 \mathrm{mM}$ Tris- $\mathrm{HCl}$, $100 \mathrm{mM} \mathrm{NaCl}, 50 \mathrm{mM} \mathrm{NaF}, 5 \mathrm{mM} \mathrm{Na}_{4} \mathrm{P}_{2} \mathrm{O}_{7}, 1 \%$ Triton X-100, Sigma, Castle Hill, NSW, Australia) with phenylmethylsulphonyl fluoride (PMSF, Sigma) and a protease inhibitor cocktail (Sigma) (both 1:100). CCL2 and CCL5 protein concentrations in cell lysates and culture media were determined using standard ELISA kits (Quantikine, R\&D systems) and normalised to the total protein content.

\section{Western Blotting}

Cell pellets were resuspended in equal volumes $(60 \mu \mathrm{l})$ of RIPA buffer (50 $\mathrm{mM}$ Tris- $\mathrm{HCl}, 1 \%$ sodium-deoxycholate, $1 \mathrm{mM} \mathrm{NaCl}, 1 \mathrm{mM}$ EDTA-Na ${ }_{2}, 1$ mM PMSF, $0.1 \%$ SDS and $1 \%$ Triton X-100, Sigma) and homogenised by passage through a pipette six times. For assessment of NF-kB (p65 subunit), the nuclear fraction was isolated using Nuclear Extraction Kit (Thermo Scientific). Equal amounts of protein were separated on a $4-12 \%$ BisTris gel (Invitrogen, Mulgrave, Victoria, Australia) by electrophoresis, then electro-transferred onto nitrocellulose membranes (Invitrogen) using iBlot (Invitrogen). Membranes were probed with primary antibodies against the chemokine receptors CCR2 (1:500, ab21667) and CX ${ }_{3}$ CR1 (1:1000, ab8021) (Abcam, Cambridge, MA, USA), the chemokine CX $_{3}$ CL1 (1:1000, \#2099, ProSci Inc., Poway, CA, USA) and the signaling proteins: $p \mathrm{l}_{\kappa} \mathrm{B} \alpha(1: 1000$, \#28595), IкB $\alpha$ (1:1000, A841), pAkt (1:300, \#40585), Akt (1:300, \#9272) 
(Cell Signaling Technology, Danvers, MA, USA) as well as pERK (1:1000, MAB1018), ERK (1:400, MAB1576) (R\&D Systems, Minneapolis, MN, USA), p65 (1:500, sc372, Santa Cruz, Santa Cruze, CA, USA) and PI3K (1:5000, \#610045, BD Bioscience, San Jose, CA, USA). This was then followed by incubation with secondary goat anti-mouse $(1: 1000, \mathrm{sc} 2030)$ or goat antirabbit (1:1000, sc2005) HRP conjugated antibodies (Santa Cruz). Equal protein loading was confirmed by $\beta$-actin (1:5000, sc81178, Santa Cruz). Protein levels were quantified from digitized images of the nitrocellulose membranes using ImageJ software and expressed relative to $\beta$-actin as arbitrary units (AU).

\section{SiRNA knockdown}

HUVSMCs were cultured in 6 well plates to $30 \%$ confluency. 16 hrs later, transfection reagent mixture was prepared using Lipofectamine 2000 (Invitrogen) in serum free Dulbecco's Modified Eagles Medium (DMEM, Lonza, Mt Waverley, Victoria, Australia). The siRNAs were used at a final concentration of $80 \mu \mathrm{M}$ (Qiagen). Fluorescein-labelled dsRNA oligomer was used to visualise transfection efficiency (Qiagen). The cells were washed with PBS and incubated at $37^{\circ} \mathrm{C}$ for $5 \mathrm{hrs}$ with siRNA transfection reagent mixtures. The transfection reagent mixture was then replaced by smooth muscle cell growth medium (Cell Applications) and incubated for a further $72 \mathrm{hrs}$. The cells were then incubated with $\mathrm{rHDL}(600 \mu \mathrm{g} / \mathrm{ml}$, final apoA-I concentration) or PBS for $16 \mathrm{hrs}$, washed twice with PBS and stimulated with TNF- $\alpha$ (final concentration $1 \mathrm{ng} / \mathrm{ml}$ ) (R\&D Systems) for $4 \mathrm{hrs}$. After stimulation, the cells were washed with PBS before harvesting.

\section{Proliferation assay}

HUVSMCs were seeded at a density of $1.5 \times 10^{5}$ cells per well (6 well plate). $24 \mathrm{hrs}$ after seeding, the cells were incubated with DMEM containing $0.1 \%$ Fetal Calf Serum (FCS, v/v) for $48 \mathrm{hrs}$. The cells were then incubated with rHDL, apoA-I, nHDL (all $600 \mu \mathrm{g} / \mathrm{ml}$, final apoA-I concentration), phospholipid vesicles $(2 \mu \mathrm{M})$, cyclodextrin $(54 \mu \mathrm{M})$ or PBS (Control) for $16 \mathrm{hrs}$. Following this, cells were washed and stimulated with $1 \mathrm{ng} / \mathrm{ml}$ TNF- $\alpha$ and 5-ethynyl2'-deoxyuridine (EdU, $40 \mu \mathrm{M}$, final concentration) and in some experiments PDGF-BB (20 ng/ml, Sigma-Aldrich, Sydney, Australia) for another $48 \mathrm{hrs}$. Cells were then harvested by trypsinisation (Trypsin EDTA, Lonza), fixed and stained according to the "Click-IT" proliferation protocol (Invitrogen). HUVSMC proliferation was measured as EdU incorporation by flow cytometry (Beckman Coulter FC500 using MXP software, Gladesville, NSW, Australia). 


\section{Statistical analyses}

Data are presented as mean \pm the standard error of the mean (SEM). All statistical analyses were performed using the Prism program (GraphPad Software Inc, San Diego, U.S.). Differences between treatment groups were evaluated for statistical significance with two-tailed, unpaired Student's t-test. Significance was accepted at the level of $p<0.05$. \# denotes a significant difference compared to non-treated cells. ${ }^{*}$ denotes a significant difference to cells incubated with TNF- $\alpha$ only. ${ }^{\# / *} p<0.05,{ }^{\# / * *} p<0.01,{ }^{\# \# / * * *} p<0.001$. 


\section{Results}

\section{Reconstituted HDL inhibit the expression of chemokines CCL2, CCL5 and $\mathrm{CX}_{3}$ CL1 in SMCs}

Culture medium and SMC lysates were analyzed as described in "Methods" for changes in CCL2, CCL5 and $\mathrm{CX}_{3} \mathrm{CL} 1$ protein and mRNA levels. Incubation with TNF- $\alpha$ significantly increased CCL2 protein levels in the cell lysates (Fig. 1A) but not in culture media (Fig. 1B). When the cells were pre-incubated with rHDL, there were significant reductions in CCL2 protein levels in the cell lysates (54\%, Fig. 1A) and culture media (62\%, Fig. 1B), compared to cells stimulated with TNF- $\alpha$ only. Consistent with the observed increase in CCL2 protein levels, TNF- $\alpha$ incubation also caused an increase in CCL2 mRNA levels (Fig. 1C) and when cells were pre-incubated with rHDL CCL2 mRNA levels were significantly reduced, compared to TNF- $\alpha$ only control cells.

Stimulation with TNF- $\alpha$ also caused a significant increase in CCL5 in cell lysates (Fig. 1D), culture media (Fig. 1E) and CCL5 mRNA levels (Fig. 1F), however, in cells that were pre-incubated with rHDL there was significantly less CCL5 protein in lysates and media and less CCL5 mRNA, compared to TNF- $\alpha$ only control cells. Similar to CCL2 and CCL5, incubation with TNF- $\alpha$ elevated $\mathrm{CX}_{3} \mathrm{CL} 1$ protein levels in cell lysates and $\mathrm{CX}_{3} \mathrm{CL} 1 \mathrm{mRNA}$ levels (Fig. 1G-H). Pre-incubation of cells with rHDL significantly suppressed these elevations in $\mathrm{CX}_{3} \mathrm{CL} 1$ protein and mRNA, compared to TNF- $\alpha$ only control cells. As $\mathrm{CX}_{3} \mathrm{CL} 1$ is predominantly a membrane-bound chemokine we were unable to detect it in the culture media. 


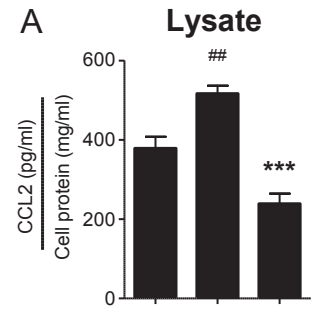

rHDL:
TNF- $\alpha$ :

D

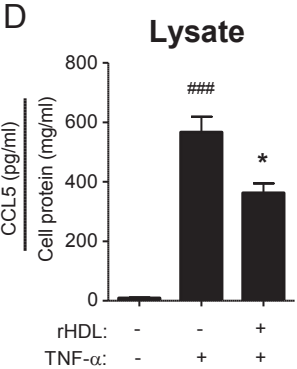

G

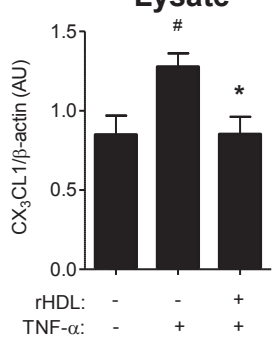

Media

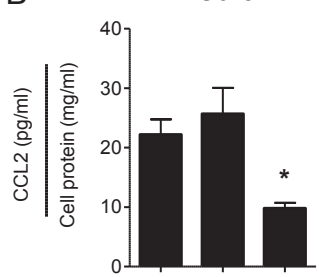

$\begin{array}{rlll}\text { rHDL: } & - & - & + \\ \text { TNF- } \alpha: & - & + & +\end{array}$

$\mathrm{E}$

Media

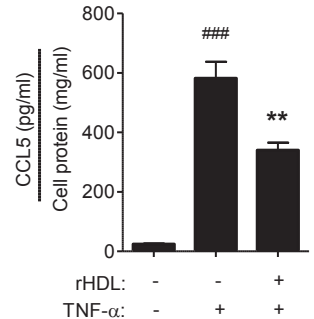

$\mathrm{H}$

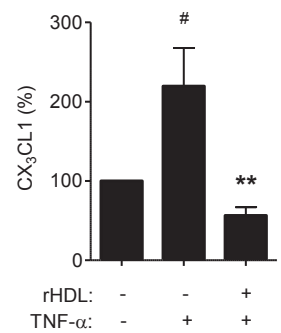

C

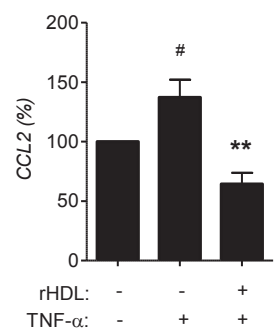

$\mathrm{F}$

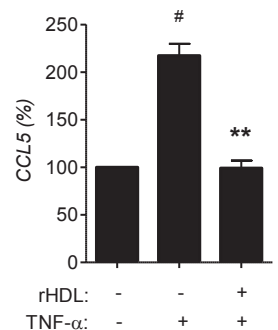

Figure 1. rHDL reduce CCL2, CCL5 and $C X_{3} C L 1$ protein and mRNA levels in SMCs. HUVSMCs were pre-incubated with rHDL $(600 \mu \mathrm{g} / \mathrm{ml}$, final apoA-I concentration) or PBS for $16 \mathrm{hrs}$ then stimulated with TNF-a $(1 \mathrm{ng} / \mathrm{ml})$ for $4 \mathrm{hrs}$. CCL2 (A-B) and CCL5 (D-E) protein levels in cell lysates and media were quantified using ELISAs. Changes in $\mathrm{CX}_{3} \mathrm{CL} 1$ protein levels in cell lysates were determined using Western blotting (G). mRNA levels were quantified using real-time PCR $(\mathbf{C}, \mathbf{F}, \mathbf{H})$. Results are expressed as mean \pm SEM. ${ }^{p} \mathrm{p}<0.05$, compared to non-treated control cells. ${ }^{*} p<0.05$, compared to cells incubated with TNF- $\alpha$ only. 


\section{The effect of apoA-I, phospholipid vesicles and nHDL on SMC chemokine expression}

Isolated human plasma nHDL and the components of rHDL (apoA-I and phospholipid vesicles) were tested for their effects on SMC chemokine expression. Incubation of SMCs with rHDL, apoA-I, phospholipid vesicles and $\mathrm{nHDL}$ had no effect on basal CCL2, CCL5 or $\mathrm{CX}_{3} \mathrm{CL} 1$ protein levels in cell lysates (Fig. 2A, C and E). However, in cells that were stimulated with TNF- $\alpha$, pre-incubation with apoA-I significantly reduced CCL2 protein levels. This reduction in CCL2 was not as great as when cells were pre-incubated with rHDL. Pre-incubation with phospholipid vesicles and $\mathrm{nHDL}$ had no effect on CCL2 levels in TNF- $\alpha$ stimulated cells. When SMCs were pre-incubated with rHDL, phospholipid vesicles and $\mathrm{nHDL}$, followed by stimulation with TNF- $\alpha$, it caused significant reductions in CCL5 protein levels in cell lysates. ApoA-I had no effect on CCL5 protein levels and neither did ApoA-I, phospholipid vesicles and $\mathrm{nHDL}$ have an effect on $\mathrm{CX}_{3} \mathrm{CL} 1$ protein levels. Only $\mathrm{rHDL}$ had an inhibitory effect on $\mathrm{CX}_{3} \mathrm{CL} 1$, when compared to TNF- $\alpha$ stimulated SMCs, confirming the findings of Fig. $1 \mathrm{G}$.

\section{The effect of cholesterol efflux on SMC chemokine expression}

Cholesterol efflux is one of the main functions of HDL. We therefore assessed its role in rHDL-mediated SMC chemokine inhibition using the cholesterol acceptor cyclodextrin. Incubation of SMCs with rHDL and cyclodextrin had no effect on basal levels of CCL2, CCL5 and CX ${ }_{3}$ CL1 (Fig. 2B, D and F). Pre-incubation with cyclodextrin, followed by stimulation with TNF- $\alpha$ had no effect on CCL2 or $\mathrm{CX}_{3} \mathrm{CL} 1$ protein levels, however there was a significant reduction in CCL5 (Fig. 2D). This reduction in CCL5 was not as great as when cells were pre-incubated with rHDL. 
A

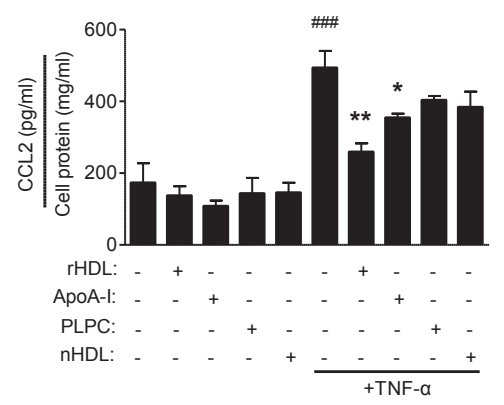

C

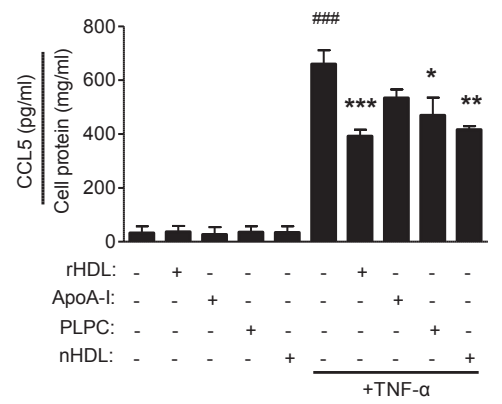

$\mathrm{E}$

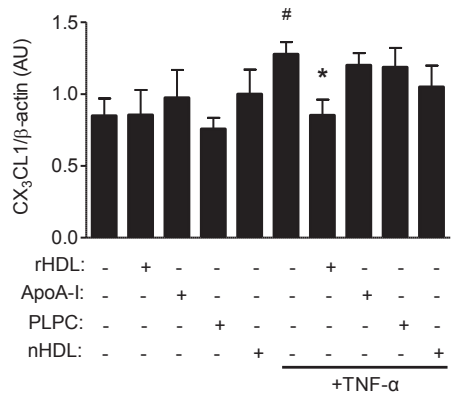

B

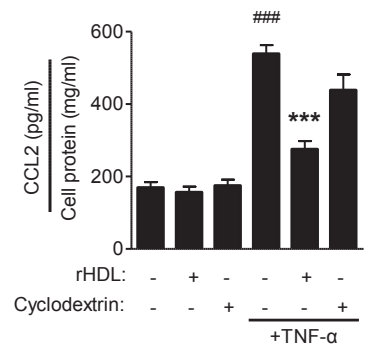

D

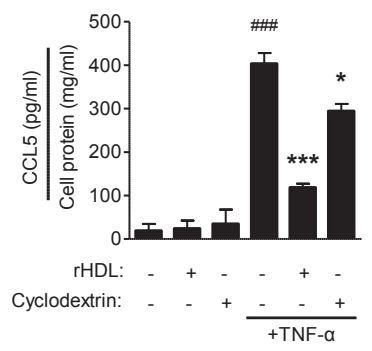

$\mathrm{F}$

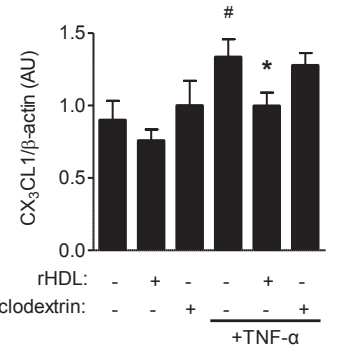

Figure 2. The effect of apoA-I, phospholipid vesicles, nHDL and cholesterol efflux on SMC chemokine expression. HUVSMCs were pre-incubated with rHDL, apoA-I, nHDL (all $600 \mu \mathrm{g} / \mathrm{ml}$, final apoA-I concentration), phospholipid vesicles (PLPC, $2 \mu \mathrm{M}$ ), cyclodextrin (54 $\mu \mathrm{M})$ or PBS for $16 \mathrm{hrs}$, then stimulated with TNF- $\alpha(1 \mathrm{ng} / \mathrm{ml})$ for $4 \mathrm{hrs}$. CCL2 (A-B) and CCL5 (C-D) protein levels in cell lysates were quantified using ELISAs. Changes in $\mathrm{CX}_{3} \mathrm{CL} 1$ protein levels were determined using Western blotting (E-F). Results are expressed as mean \pm SEM. ${ }^{*} p<0.05$, compared to non-treated control cells. ${ }^{*} p<0.05$, compared to cells incubated with TNF- $\alpha$ only. 


\section{SR-B1 mediates the inhibition of CCL2, CCL5 and CX ${ }_{3}$ CL1 by rHDL} To determine whether the ATP-binding cassette transporters ABCA1 and ABCG1 or the scavenger receptor SR-B1 mediate the inhibitory effects of rHDL on chemokine expression, siRNAs were used to knockdown these proteins in SMCs. Western blotting was used to confirm siRNA knockdown efficiency. We were able to achieve efficient transfection in SMCs and a subsequent $>90 \%$ inhibition of $A B C A 1$ protein as well as complete knockdown of ABCG1 and SR-B1 proteins (Suppl. Fig. 1). When scrambled siRNA control cells were incubated with TNF- $\alpha$ there was a significant increase in CCL2 protein levels (Fig. 3A). Pre-incubation of scrambled siRNA control cells with rHDL inhibited CCL2 protein levels, as in Fig. 1A. Knockdown of $A B C A 1$ and ABCG1 did not affect the ability of rHDL to inhibit the TNF- $\alpha$ induced increase in CCL2. When SR-B1 was knocked down, the inhibitory effect of rHDL on CCL2 protein levels was attenuated.

Incubation of scrambled siRNA cells with TNF- $\alpha$ significantly increased CCL5 protein levels, as measured by ELISA (Fig. 3B). When the siRNA control cells were pre-incubated with rHDL, CCL5 levels were significantly suppressed compared to TNF- $\alpha$ only controls. siRNA knockdown of ABCA1 or ABCG1 did not affect the ability of rHDL to inhibit CCL5. Inhibition of CCL5 by $\mathrm{rHDL}$ in the ABCA1 and ABCG1 knockdown cells was similar to siRNA control cells. The siRNA knockdown of SR-B1, however, abolished the ability of rHDL to inhibit expression of CCL5.

Incubation of scrambled siRNA cells with TNF- $\alpha$ significantly increased $\mathrm{CX}_{3} \mathrm{CL} 1$, as determined by Western blotting (Fig. 3C). Pre-incubation of scrambled siRNA control cells with $\mathrm{rHDL}$ inhibited $\mathrm{CX}_{3} \mathrm{CL} 1$ protein levels. Inhibition of $\mathrm{CX}_{3} \mathrm{CL} 1$ in the $\mathrm{ABCA} 1$ and $\mathrm{ABCG} 1$ knockdown cells was comparable to what was observed for the siRNA control cells. Finally, siRNA knockdown of SR-B1, significantly attenuated the ability of rHDL to inhibit expression of $\mathrm{CX}_{3} \mathrm{CL} 1$. 
A

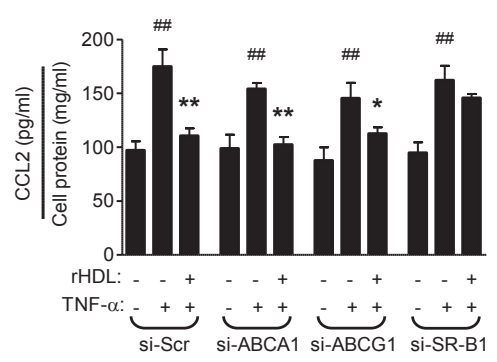

C

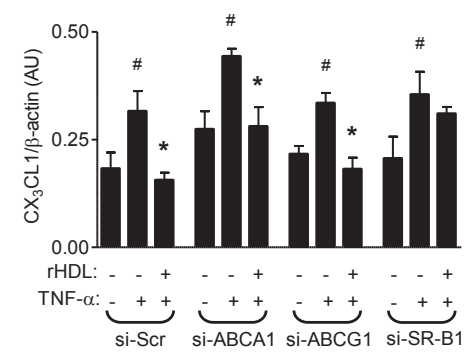

B

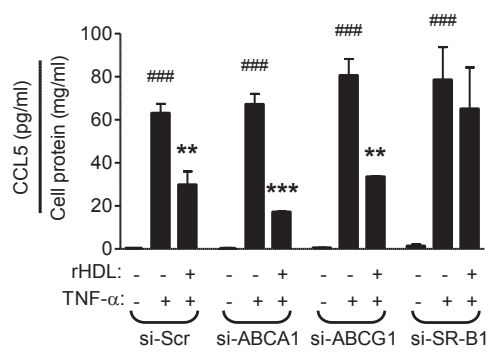

Figure 3. rHDL inhibit SMC CCL2, CCL5 and CX ${ }_{3}$ CL1 expression via SR-B1. HUVSMCs were transfected with scrambled (Scr), ABCA1, ABCG1 or SR-B1 siRNA. After $72 \mathrm{hrs}$, the cells were incubated for $16 \mathrm{hrs}$ with $\mathrm{rHDL}(600 \mu \mathrm{g} / \mathrm{ml}$, final apoA-I concentration) or PBS and then stimulated with TNF- $\alpha(1 \mathrm{ng} / \mathrm{ml})$ for $4 \mathrm{hrs}$. Changes in CCL2 (A) and CCL5 (B) protein levels were quantified by ELISA. Western blotting was used to determine changes in $\mathrm{CX}_{3} \mathrm{CL} 1$ protein $(\mathbf{C})$. Results are expressed as mean \pm SEM. ${ }^{p}<0.05$, compared to non-treated control cells. ${ }^{*} p<0.05$, compared to cells incubated with TNF- $\alpha$ only.

\section{Reconstituted HDL suppress the NF-KB and PI3K/Akt pathways in SMCs}

NF-KB regulates chemokine expression. In order to determine the effect of $\mathrm{rHDL}$ on the NF-KB activation pathway, the protein levels of the active subunit of NF-kB, p65, were determined in SMC nuclear extracts. Stimulation with TNF- $\alpha$ increased p65 protein levels (Fig. 4A), compared to control cells. However, pre-incubation with rHDL significantly suppressed this elevation in p65, relative to cells stimulated with TNF- $\alpha$ only. Stimulation with TNF- $\alpha$ increased the phosphorylation of $\mathrm{I} \mathrm{KB} \alpha$, part of the NF-KB activation pathway and pre-incubation of SMCs with $\mathrm{rHDL}$ significantly decreased phosphorylated IKBa, when compared to TNF- $\alpha$ stimulated cells (Fig. 4B). Taken together, these results demonstrate that $\mathrm{rHDL}$ inhibit IKBa phosphorylation and the subsequent translocation of p65 to the nucleus. 
Stimulation of SMCs with TNF- $\alpha$ for 10 min caused an increase in $\mathrm{PI} 3 \mathrm{~K}$ protein levels. PI3K protein levels were significantly inhibited when SMCs were preincubated with rHDL (Fig. 4C). Stimulation with TNF-a did not significantly change Akt phosphorylation, however, pre-incubation with rHDL reduced Akt phosphorylation, compared to cells stimulated with TNF-a only (Fig. 4D).

A
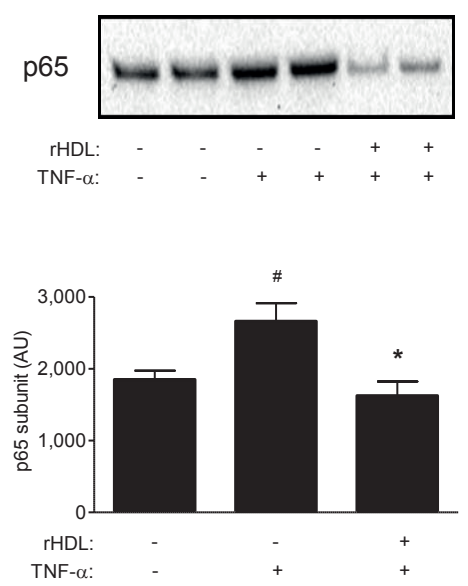

C
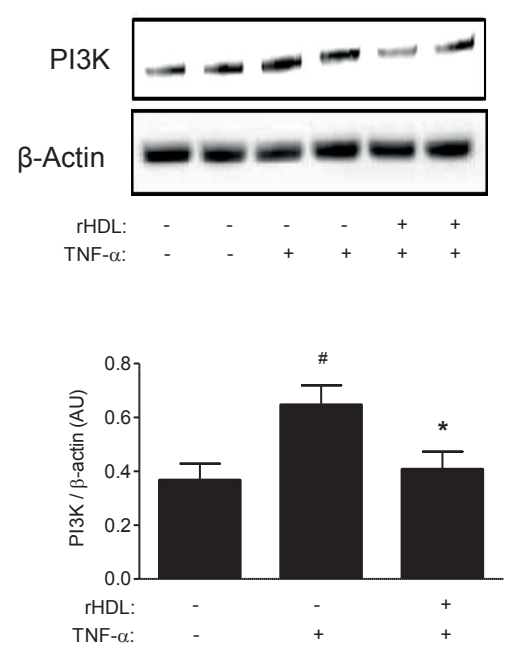

B
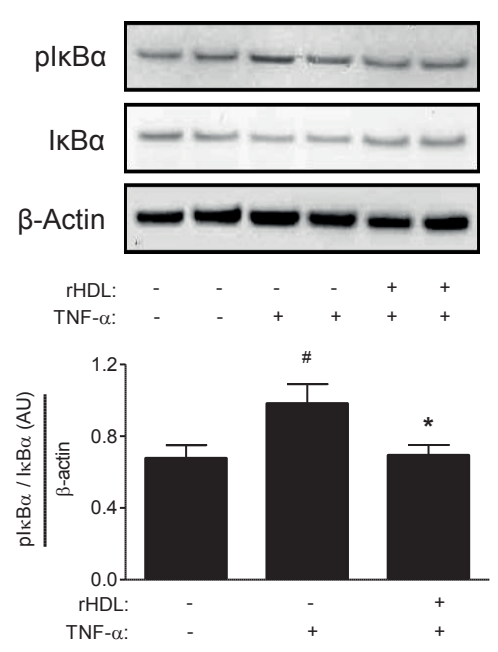

D
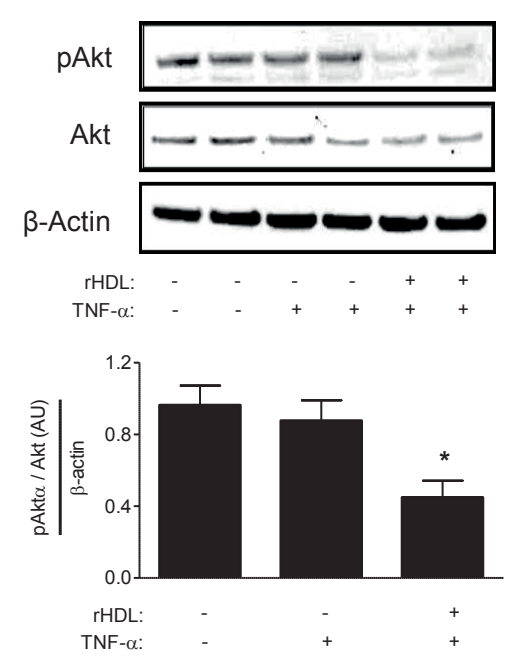
Figure 4. rHDL inhibit the NF-KB - PI3K/Akt pathway in SMCs. (A-B). HUVSMCs were incubated with $\mathrm{rHDL}(600 \mu \mathrm{g} / \mathrm{ml}$, final apoA-I concentration) or PBS for $16 \mathrm{hrs}$ and then stimulated with TNF- $\alpha(1 \mathrm{ng} / \mathrm{ml})$ for $4 \mathrm{hrs}$. Using Western blotting, p65 protein levels were assessed in nuclear extracts. Phosphorylated $1 \mathrm{kB \alpha}(\mathrm{pl \kappa B \alpha})$ was measured in whole cell lysates and expressed relative to total IKBa and $\beta$-actin. (C-D) HUVSMCs were pre-incubated with $\mathrm{rHDL}(600 \mu \mathrm{g} / \mathrm{ml}$, final apoA-I concentration) or PBS for $16 \mathrm{hrs}$ and stimulated with TNF- $\alpha(1 \mathrm{ng} / \mathrm{ml})$ for $10 \mathrm{~min}$. PI3K and phosphorylated Akt (pAkt) protein levels were assessed by Western blotting and expressed relative to $\beta$-actin or total Akt and $\beta$-actin, respectively. Results are presented as mean \pm SEM. ${ }^{*} p<0.05$, compared to non-treated control cells. ${ }^{*} p<0.05$, compared to cells incubated with TNF- $\alpha$ only.

\section{Mechanisms for rHDL-induced inhibition of p65 in SMCs}

When SMCs were incubated with rHDL and phospholipid vesicles there were significant reductions in the basal levels of p65 protein in nuclear extracts (Fig. 5A), compared to control cells. There were no reductions in basal p65 protein levels following incubation with apoA-I or nHDL. Pre-incubation with apoA-I, phospholipid vesicles and $\mathrm{nHDL}$ had no effect on the protein levels of p65 when cells were stimulated with TNF- $\alpha$. However, as also demonstrated in Fig. 4A, pre-incubation with rHDL in TNF- $\alpha$ stimulated cells reduced p65 protein levels in nuclear extracts.

Incubation of SMCs with cyclodextrin did not affect basal levels of p65 in nuclear extracts (Fig. 5B). However, in TNF- $\alpha$ stimulated cells, preincubation with cyclodextrin significantly reduced $\mathrm{p} 65$, when compared to TNF- $\alpha$ only stimulated cells. Indicating a role for cholesterol efflux in rHDLinduced inhibition of SMC chemokine expression and this is consistent with the inhibitory effect of cyclodextrin on CCL5 (Fig. 2E). The importance of SR-B1 was also assessed. Cells transfected with SR-B1 siRNA, had similar p65 protein levels as control non-transfected cells and cells transfected with scrambled siRNA. When SR-B1 transfected cells were stimulated with TNF- $\alpha$ there was an increase in p65 protein levels (Fig. 5C). Transfection of SR-B1 siRNA completely attenuated the inhibitory effects of $\mathrm{rHDL}$ on p65 protein levels in TNF- $\alpha$ stimulated SMCs. This supports a role for SR-B1 in the inhibitory effects of rHDL on SMC chemokine expression, which is consistent with Fig. 3 where SR-B1 siRNA prevented rHDL-induced inhibition of SMC chemokines. 
A

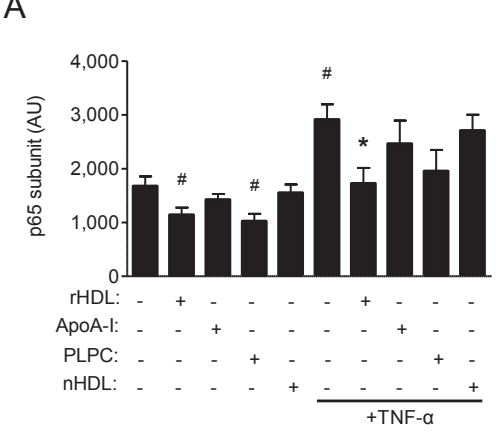

C

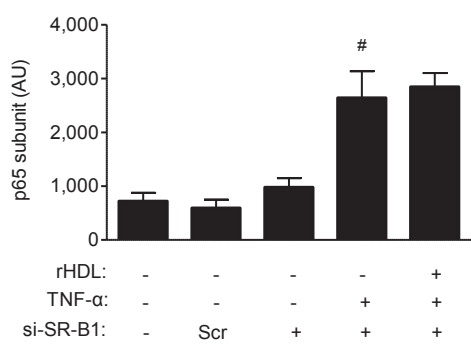

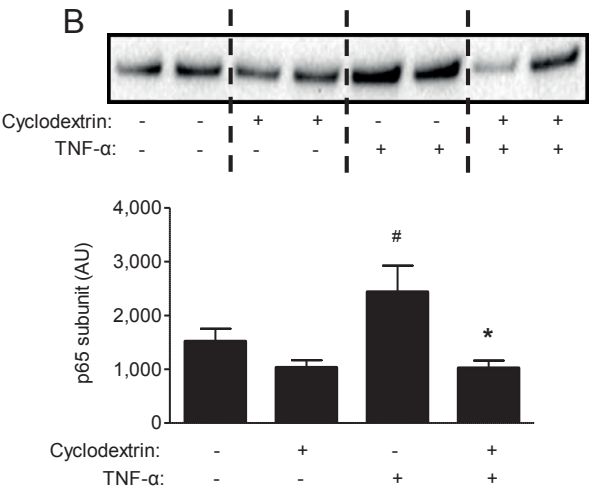

Figure 5. Mechanisms of rHDL-induced inhibition of p65 in SMCs. (A-B) HUVSMCs were pre-incubated with $\mathrm{rHDL}$, apoA-I, nHDL (all $600 \mu \mathrm{g} / \mathrm{ml}$, final apoA-I concentration), phospholipid vesicles (PLPC, $2 \mu \mathrm{M})$, cyclodextrin $(54 \mu \mathrm{M})$ or PBS for $16 \mathrm{hrs}$, then stimulated with TNF- $\alpha(1 \mathrm{ng} / \mathrm{ml})$ for $4 \mathrm{hrs}$. (C) HUVSMCs were transfected with scrambled (Scr) and SRB1 siRNA. After $72 \mathrm{hrs}$, the cells were incubated for $16 \mathrm{hrs}$ with $\mathrm{rHDL}(600 \mu \mathrm{g} / \mathrm{ml}$, final apoA-I concentration) or PBS and then stimulated with TNF- $\alpha(1 \mathrm{ng} / \mathrm{ml})$ for $4 \mathrm{hrs}$. Western blotting was used to assess p65 protein levels in nuclear extracts. Results are presented as mean \pm SEM. ${ }^{*} p<0.05$, compared to non-treated control cells. ${ }^{*} p<0.05$, compared to cells incubated with TNF-a only. 


\section{rHDL inhibit SMC proliferation}

The effect of rHDL on SMC proliferation was determined using the "Click-IT" proliferation assay. Stimulation of SMCs with TNF- $\alpha$ increased proliferation, compared to control cells (Fig. 6A). When SMCs were incubated with rHDL before stimulation with TNF- $\alpha$, proliferation was strikingly reduced, compared to TNF- $\alpha$ only treated SMCs. Furthermore, rHDL was found to inhibit SMC proliferation at lower concentrations (inset Fig. 6A). Pre-incubation of cells with rHDL at final apoA-I concentrations of 6.5 and $13 \mu \mathrm{M}$ significantly inhibited SMC proliferation.

Incubation with rHDL, apoA-I and phospholipid vesicles increased SMC proliferation in non-TNF- $\alpha$ stimulated cells (Fig. 6B). nHDL had no effect on SMC proliferation in non-stimulated cells. However, pre-incubation with rHDL, apoA-I and phospholipid vesicles inhibited TNF- $\alpha$ stimulated SMC proliferation, when compared to cells that were incubated with TNF- $\alpha$ only. The role of cholesterol efflux was also assessed on SMC proliferation. Cyclodextrin had no effect on SMC proliferation in non-TNF- $\alpha$ stimulated cells or on TNF- $\alpha$ stimulated cells (Fig. 6C).

Knockdown of ABCA1, ABCG1 and SR-B1 with siRNA was used to determine their importance in mediating the inhibitory actions of rHDL on SMC proliferation (Fig. 6D). In scrambled siRNA transfected control cells, stimulation with TNF- $\alpha$ increased SMC proliferation and when they were preincubated with rHDL, proliferation was inhibited. When ABCA1 and ABCG1 were knocked down, the extent of inhibition of proliferation by rHDL was not affected. When SR-B1 was knocked down, the inhibitory effect of rHDL on SMC proliferation was ameliorated.

Incubation with platelet-derived growth factor (PDGF)-BB was used to determine if rHDL could inhibit SMC proliferation under stimuli different to TNF- $\alpha$ (Suppl. Fig. 2). PDGF increased SMC proliferation, but pre-treatment with rHDL, apoA-I, phospholipid vesicles and $\mathrm{nHDL}$ had no effect on PDGFstimulated proliferation. 


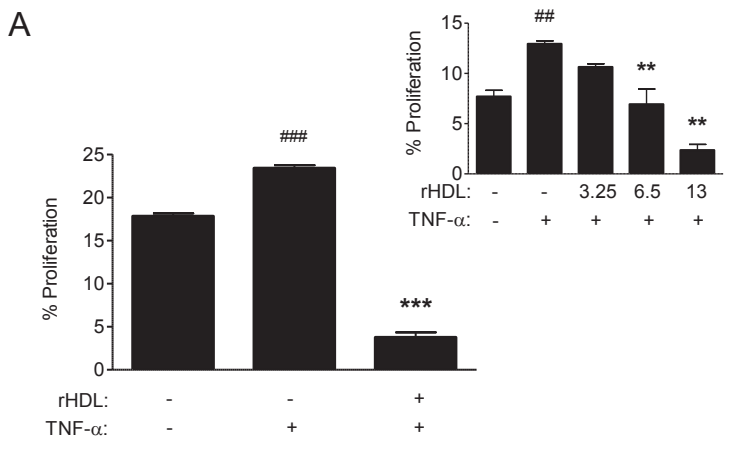

C

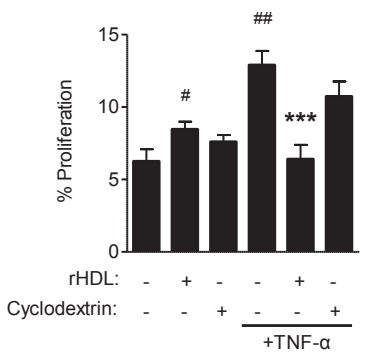

B

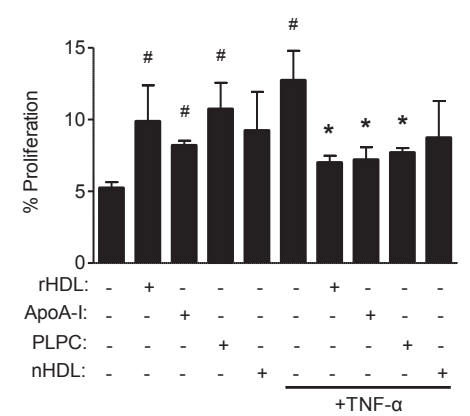

D

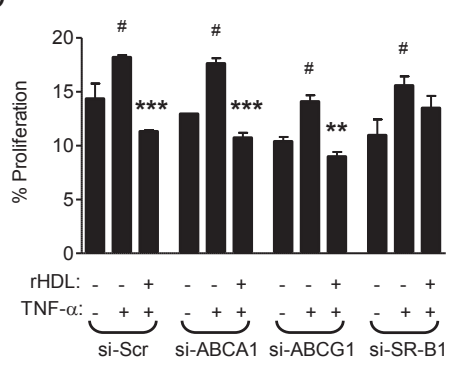

Figure 6. rHDL inhibit SMC proliferation. (A-C) HUVSMCs were serum starved by incubation with DMEM containing $0.1 \%$ Fetal Calf Serum (FCS, v/v) for $48 \mathrm{hrs}$. The cells were then incubated with rHDL, apoA-I, nHDL (all $600 \mu \mathrm{g} / \mathrm{ml}$, final apoA-I concentration), phospholipid vesicles (PLPC, $2 \mu \mathrm{M})$, cyclodextrin $(\mathrm{CD}, 54 \mu \mathrm{M})$ or PBS for 16 hrs prior to stimulation with $1 \mathrm{ng} / \mathrm{ml} \mathrm{TNF- \alpha}$ and EdU $(40 \mu \mathrm{M})$ for a further $48 \mathrm{hrs}$. The cells were then fixed and stained according to the "Click-IT" proliferation protocol. (D) SMC proliferation was determined following transfection of HUVSMCs with scrambled (Scr), ABCA1, ABCG1 or SR-B1 siRNA. Cells were then treated with $\mathrm{rHDL}$ or PBS and stimulated with TNF- $\alpha$ before proliferation was assessed as described above. Results are presented as mean \pm SEM. ${ }^{p}<<0.05$, compared to non-treated control cells. ${ }^{*} p<0.05$, compared to cells incubated with TNF- $\alpha$ only. 


\section{rHDL inhibit ERK phosphorylation}

Chemokines stimulate SMC proliferation via increased phosphorylation of $\mathrm{ERK}_{1 / 2}{ }^{8}$. Incubation with rHDL was found to increase basal levels of ERK phosphorylation, compared to control cells. Stimulation of SMCs with TNF- $\alpha$ also increased phosphorylation of ERK. However, when cells were preincubated with $\mathrm{rHDL}$, ERK phosphorylation was significantly decreased, compared to cells stimulated with TNF- $\alpha$ only (Fig. 7A).

When cells transfected with scrambled siRNA were incubated with rHDL before stimulation with TNF- $\alpha$, ERK phosphorylation was significantly reduced (Fig. 7B). The siRNA knockdown of ABCA1 and ABCG1 did not affect the inhibitory actions of rHDL on pERK protein levels, as the extent of inhibition was similar to the scrambled control. However, when SR-B1 was knocked down, this suppression was ameliorated.

A
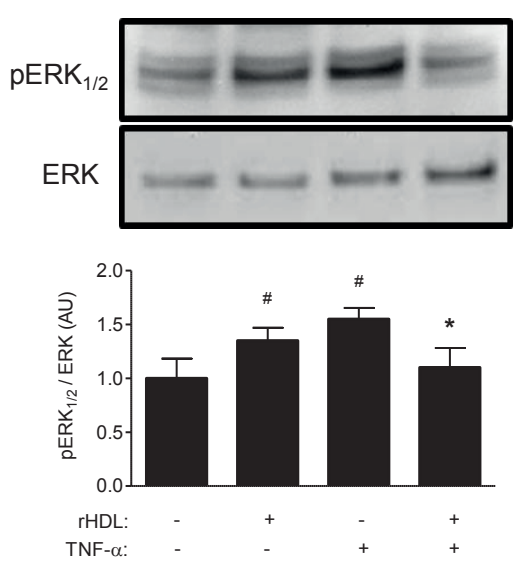

B

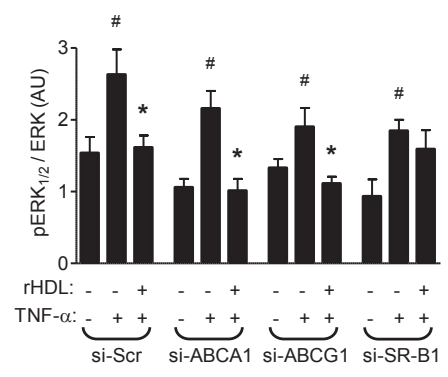

Figure 7. rHDL inhibit ERK phosphorylation. (A) HUVSMCs were pre-incubated with rHDL $(600 \mu \mathrm{g} / \mathrm{ml}$, final apoA-I concentration) or PBS for $16 \mathrm{hrs}$ and stimulated with TNF- $\alpha$ (1 ng/ $\mathrm{ml}$ ) for $10 \mathrm{~min}$. (B) HUVSMCs were transfected with scrambled (Scr), ABCA1, ABCG1 or SR-B1 siRNA for 72 hrs. Cells were then treated with rHDL or PBS for $16 \mathrm{hrs}$ and stimulated with TNF- $\alpha$. Phosphorylated ERK $\mathrm{E}_{1 / 2}\left(\mathrm{pERK}_{1 / 2}\right)$ protein levels were assessed using Western blotting and expressed relative to total ERK. Results are expressed as mean \pm SEM. ${ }^{p}<0.05$, compared to non-treated control cells. ${ }^{*} p<0.05$, compared to cells incubated with TNF- $\alpha$ only. 


\section{The role of chemokines in SMC proliferation}

SMCs were transfected with siRNAs to knockdown the expression of CCL2, CCL5 and $\mathrm{CX}_{3} \mathrm{CL} 1$. When scrambled siRNA transfected cells were stimulated with TNF- $\alpha$, SMC proliferation was elevated (Suppl. Fig. 3). In SMCs transfected with CCL2, CCL5 and $\mathrm{CX}_{3} \mathrm{CL} 1$ siRNAs, prior to TNF- $\alpha$ stimulation, SMC proliferation was reduced, compared to TNF- $\alpha$ only cells. This confirms their importance in inflammation-driven SMC proliferation ${ }^{4-8}$.

\section{The effect of rHDL on SMC chemokine receptors CCR2 and $\mathrm{CX}_{3} \mathrm{CR} 1$}

CCR2 is the chemokine receptor for CCL2. Western blot analysis showed that stimulation with TNF- $\alpha$ increased CCR2 protein levels (Fig. 8A). Incubation with rHDL decreased basal SMC CCR2 protein levels and in cells stimulated with TNF- $\alpha$. Furthermore, TNF- $\alpha$ had no effect on $\mathrm{CX}_{3} \mathrm{CR} 1$ protein levels (the receptor for $\mathrm{CX}_{3} \mathrm{CL} 1$ ), but incubation with $\mathrm{rHDL}$ significantly reduced $\mathrm{CX}_{3} \mathrm{CR} 1$ in non-stimulated and stimulated cells (Fig. 8B).

A

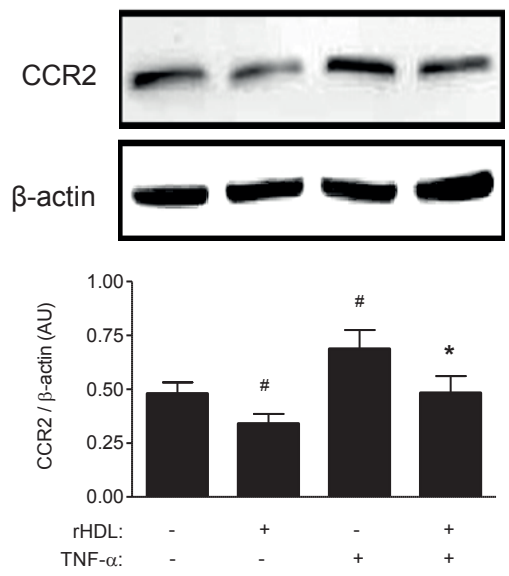

B

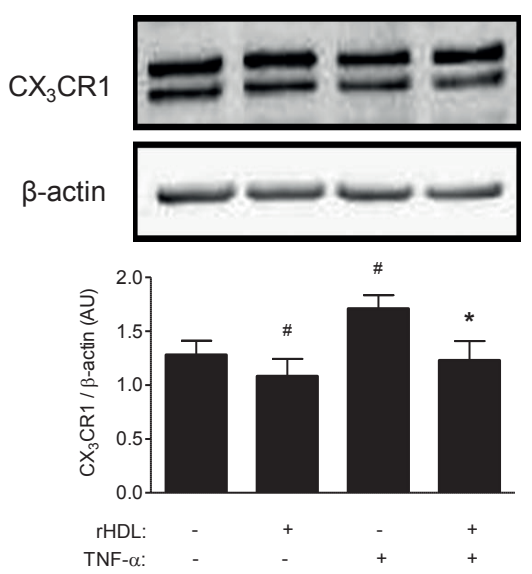

Figure 8. rHDL inhibit CCR2 and CX ${ }_{3}$ CR1 expression in SMCs. HUVSMCs were incubated with $\mathrm{rHDL}(600 \mu \mathrm{g} / \mathrm{ml}$, final apoA-I concentration) or PBS for $16 \mathrm{hrs}$ and then stimulated with TNF- $\alpha(1 \mathrm{ng} / \mathrm{ml})$ for $4 \mathrm{hrs}$. Protein levels were determined by Western blotting and expressed relative to $\beta$-actin. Results are expressed as mean \pm SEM. $\# p<0.05$, compared to non-treated control cells. ${ }^{*} p<0.05$, compared to cells incubated with TNF- $\alpha$ only. 


\section{Discussion}

Unregulated, inflammation-induced SMC proliferation is a key driver of neointimal hyperplasia and causes re-narrowing of the lumen following $\mathrm{PCl}$ such as stent implantations ${ }^{5,6}$. The chemokines CCL2, CCL5 and CX ${ }_{3}$ CL1 have all been implicated in the promotion of SMC proliferation ${ }^{4-6,8,14,26}$. HDL have potent anti-inflammatory properties ${ }^{2}$ and have previously been shown to inhibit the expression of the chemokines CCL2, CCL5 and CX ${ }_{3} \mathrm{CL} 1$ and their receptors ${ }^{17-21}$. The aim of this study was to determine whether rHDL exhibit anti-inflammatory effects in SMCs by inhibiting chemokine expression and proliferation. We have found that rHDL inhibit SMC expression of CCL2, CCL5 and $C X_{3}$ CL1 via inhibition of intracellular PI3K/Akt and NF-KB pathways. We also demonstrate that $\mathrm{rHDL}$ inhibit SMC proliferation via a reduction in ERK phosphorylation. rHDL inhibit the expression of CCR2 and $\mathrm{CX}_{3} \mathrm{CR} 1$, two chemokine receptors that are known to be critical for mediating the proliferative effects of chemokines in SMCs. Finally, SR-B1, plays a key role in mediating the inhibitory effects of rHDL on SMC chemokine expression, p65 and proliferation.

Inflammation-driven neointimal hyperplasia plays causative role in the reduction of stent patency ${ }^{14}$. The thickened neointima predominantly contains SMCs that proliferate in an unregulated manner, secrete inflammatory factors and can recruit leukocytes ${ }^{6,26}$. Accumulating evidence has pointed to an important role for chemokines in the promotion of neointimal thickening following $\mathrm{PCl}$. The chemokines CCL2, CCL5 and $\mathrm{CX}_{3} \mathrm{CL} 1$ have all been found to play important roles in SMC proliferation following vascular injury. For example, chemokine knockout mice ${ }^{5,13}$ and strategies that inhibit or increase the expression of CCL2, CCL5 and $\mathrm{CX}{ }_{3} \mathrm{CL} 1$ are found to modulate neointimal hyperplasia in surgical models ${ }^{5,13,14,26}$. Using siRNA knockdown we confirmed the importance of these chemokines in inflammation-driven SMC proliferation. We also show that rHDL inhibit the expression of the chemokines CCL2, CCL5 and $\mathrm{CX}_{3} \mathrm{CL} 1$. These later findings support our previous work and the work of others, which demonstrated rHDL inhibit these three chemokines in vitro in primary human coronary artery endothelial cells (HCAECs) and monocytes ${ }^{17,18}$ and in in vivo studies ${ }^{19,20}$. It also suggests that rHDL may protect against the development of neointimal hyperplasia and thereby prolong stent patency. This is consistent with clinical studies that find patients with higher HDL cholesterol levels (>45 mg/dL) have improved 
stent patency at one year ${ }^{27}$, and reduced major cardiac events following stent implantation ${ }^{28}$.

Vascular SMC chemokine expression is regulated via the transcription factor $\mathrm{NF}-\kappa \mathrm{B}^{4,5}$ and further upstream by the PI3K/Akt pathway ${ }^{4,8}$. The current study found that incubation of SMCs with $\mathrm{rHDL}$ reduced the level of the p65 subunit of NF-KB in the nucleus, the phosphorylation of its upstream regulator $I_{\kappa} \mathrm{B} \alpha, \mathrm{PI} \mid 3 \mathrm{~K}$ and the phosphorylation of Akt. This suggests that $\mathrm{rHDL}$ inhibit CCL2, CCL5 and $\mathrm{CX}_{3} \mathrm{CL} 1$ via suppression of the PI3K/Akt and IKB $\alpha /$ $\mathrm{NF}-\kappa \mathrm{B}$ intracellular pathways. Consistent with these findings, other studies have also found that $\mathrm{rHDL}$ is able to inhibit the NF-kB activation pathway in HCAECs and monocytes ${ }^{17,25}$. Furthermore, HDL isolated from human plasma have also been found to reduce oxidized LDL-induced elevations in NF- $\kappa B$ in rabbit femoral SMCs ${ }^{29}$. Interestingly, in contrast to our findings, $\mathrm{PI} 3 \mathrm{~K}$ was found not to be a target of HDL in that study ${ }^{29}$. This may reflect the use of the cytokine TNF- $\alpha$ to stimulate PI3K (as used previously ${ }^{4}$ ) in the present study, as opposed to the use of oxidized LDL in the earlier study, which may activate PI3K via an alternative pathway to TNF- $\alpha{ }^{29}$.

HDL interact with three cell-surface proteins: ABCA1 and ABCG1 and SR$B 1$. Although the vasculo-protective properties of HDL have been reported to be predominantly mediated via cholesterol efflux, there is mounting evidence that HDL may also confer benefit by inducing intracellular signaling events via interactions with these proteins. The current study found that rHDL mediate inhibition of SMC chemokine expression, p65 and proliferation via SR-B1. This is in agreement with other studies showing that rHDL mediate their actions through SR-B1 ${ }^{30,31}$. Work in this area has largely focused on the beneficial effects of HDL on the endothelium including: rHDL-induced elevations in endothelial cell migration ${ }^{31}$, enhanced endothelial-mediated vascular function ${ }^{30}$ and improved endothelial repair ${ }^{31,32}$, using in vitro SR-B1 siRNA silencing or knockout mouse models. In the present study, silencing SR-B1 effectively attenuated the reductions in SMC chemokine expression. Consistent with this SR-B1 mediated anti-inflammatory effect of rHDL, work by McGrath et al. found that the anti-inflammatory effects of rHDL in HCAECs were also mediated via SR-B1 through an elevation in $3 \beta$-Hydroxysteroid- $\Delta 24$ Reductase (DHCR24) ${ }^{25}$. Cholesterol efflux also appeared to have a role (although modest) in mediating the inhibitory effects on SMC chemokine expression. Incubation with the cholesterol acceptor cyclodextrin significantly reduced CCL5 protein levels and caused a non- 
significant reduction in CCL2 (19\%). When compared to rHDL, cyclodextrin was not as effective at inhibiting SMC chemokine expression, indicating that other mechanisms in addition to cholesterol efflux are mediating these inhibitory effects. Consistent with these findings, we also found that cyclodextrin suppressed p65 and cholesterol efflux is reported by others to mediate anti-inflammatory effects ${ }^{33}$. It is possible therefore that SR-B1 may mediate rHDL-induced suppression of SMC chemokine expression at least in part, by cholesterol efflux (Fig. 9).

rHDL strikingly reduced SMC proliferation in the present study. This appears to have been mediated by three possible pathways (Fig. 9): 1) via SR$\mathrm{B} 1$, which causes downstream suppression of the $\mathrm{PI} / 3 \mathrm{~K} / \mathrm{Akt} / \mathrm{/} \mathrm{KB} \alpha / \mathrm{NF}-\kappa \mathrm{B}$ pathway and inhibition of CCL2, CCL5 and $\mathrm{CX}_{3} \mathrm{CL} 1$ expression, chemokines that are known to promote SMC proliferation ${ }^{5,8,14}$ and 2) via a reduction in the expression of chemokine receptors $\mathrm{CCR} 2$ and $\mathrm{CX}_{3} \mathrm{CR} 1$, which reduces chemokine/chemokine receptor interactions and downstream activation of ERK, an important mediator of SMC proliferation. This also supports the findings of Roque et al. who reported that neointimal hyperplasia was significantly reduced in CCR2 ${ }^{-/}$mice following femoral artery injury ${ }^{13}$, suggesting a key role for CCR2 in SMC proliferation. 3) Finally, ERK is also downstream of SR-B1 ${ }^{32}$, suggesting a third possible pathway for rHDLmediated inhibition of SMC proliferation. In support of this, the present results show that silencing of SR-B1 attenuated the inhibitory effects of rHDL on SMC proliferation and ERK phosphorylation.

The current study found that cholesterol efflux did not play a role in the inhibitory effect of rHDL on SMC proliferation. This is in contrast to a recent report that found cholesterol efflux played a key role in the inhibition of hematopoietic stem cell proliferation ${ }^{34}$. The disparity in these results is likely to be due to the differences in cell-type used in the two studies and indicates that it is the reduction in chemokine expression by rHDL that is inhibiting SMC proliferation. In contrast to the inhibitory effects of rHDL on SMC proliferation in TNF- $\alpha$ stimulated cells, rHDL was able to induce SMC proliferation in nonstimulated cells. This result was supported by the increase in pERK in nonstimulated cells and is consistent with a previous report that found $\mathrm{nHDL}$ induced pERK in non-stimulated human vascular SMCs ${ }^{35}$. The current study also found that rHDL was unable to inhibit PDGF-stimulated SMC proliferation. In agreement with this, previous reports have found that the proliferative effects of chemokine $\mathrm{CX}_{3} \mathrm{CL} 1$ are not mediated via PDGF-BB 
but rather via NF-kB and epidermal growth factor (EGF) pathways ${ }^{4,8}$. Taken together, this suggests that HDL conditionally regulates SMC proliferation and ERK phosphorylation, which is dependent on the presence (or not) of a stimulus and the type of stimulus.

The current study found that there were discrepancies between the effects of HDL and cyclodextrin on SMC chemokine expression. A possible reason for this is that they remove cholesterol by different mechanisms. In the case of $\mathrm{nHDL}$, cholesterol efflux occurs via desorption of unesterified cholesterol from the plasma membrane into the aqueous phase and subsequent incorporation of the desorbed cholesterol into the phospholipid-containing acceptor particles (nHDL or rHDL) ${ }^{36}$. The much smaller size of cyclodextrin molecules, by contrast, allows them to access invaginations in caveolae, where cholesterol can associate directly with the acceptor.

Overall, the different components of rHDL (apoA-I and phospholipid vesicles) and $\mathrm{nHDL}$ isolated from human plasma had more modest or no effects on SMC chemokine expression and proliferation, when compared to rHDL. Other studies have reported similar findings and show that rHDL is more effective at inhibiting vascular cell adhesion molecule-1 (VCAM-1) than lipid-free apoA-I or phospholipid vesicles ${ }^{37,38}$. It may have been expected that $\mathrm{nHDL}$ would be more effective than rHDL at inhibiting SMC chemokine expression and proliferation, particularly as these effects appear to be mediated via SR$\mathrm{B} 1$, which is the preferred acceptor for nHDL rather than rHDL. However, a recent publication has identified that SR-B1 can act as a cholesterol sensor that then triggers intracellular signaling and is important to the actions of $\mathrm{nHDL}{ }^{39}$. Whilst rHDL only contains phospholipid initially, after incubation with the SMCs they are likely to acquire cholesterol, enabling them to then interact with SR-B1 and subsequently mediate anti-inflammatory and antiproliferative effects. In conclusion, rHDL inhibit SMC chemokine expression and proliferation. These findings suggest that strategies, which increase HDL levels may protect against inflammation-driven neointimal hyperplasia. This has important implications in the pathogenesis of restenosis, which can lead to early stent failure. 
$\rightarrow$ Mechanistic Pathways of rHDL action

- Effects via Cholesterol Efflux

(10) Unesterified

Cholesterol

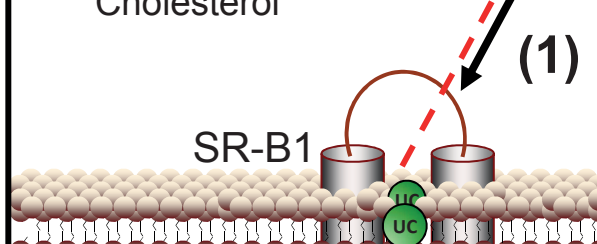

(1)

\section{rHDL}

(2)

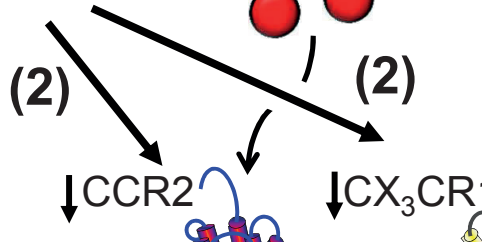

$\downarrow \mathrm{CCL} 2 \quad \downarrow \mathrm{CX}_{3} \mathrm{CL} 1$

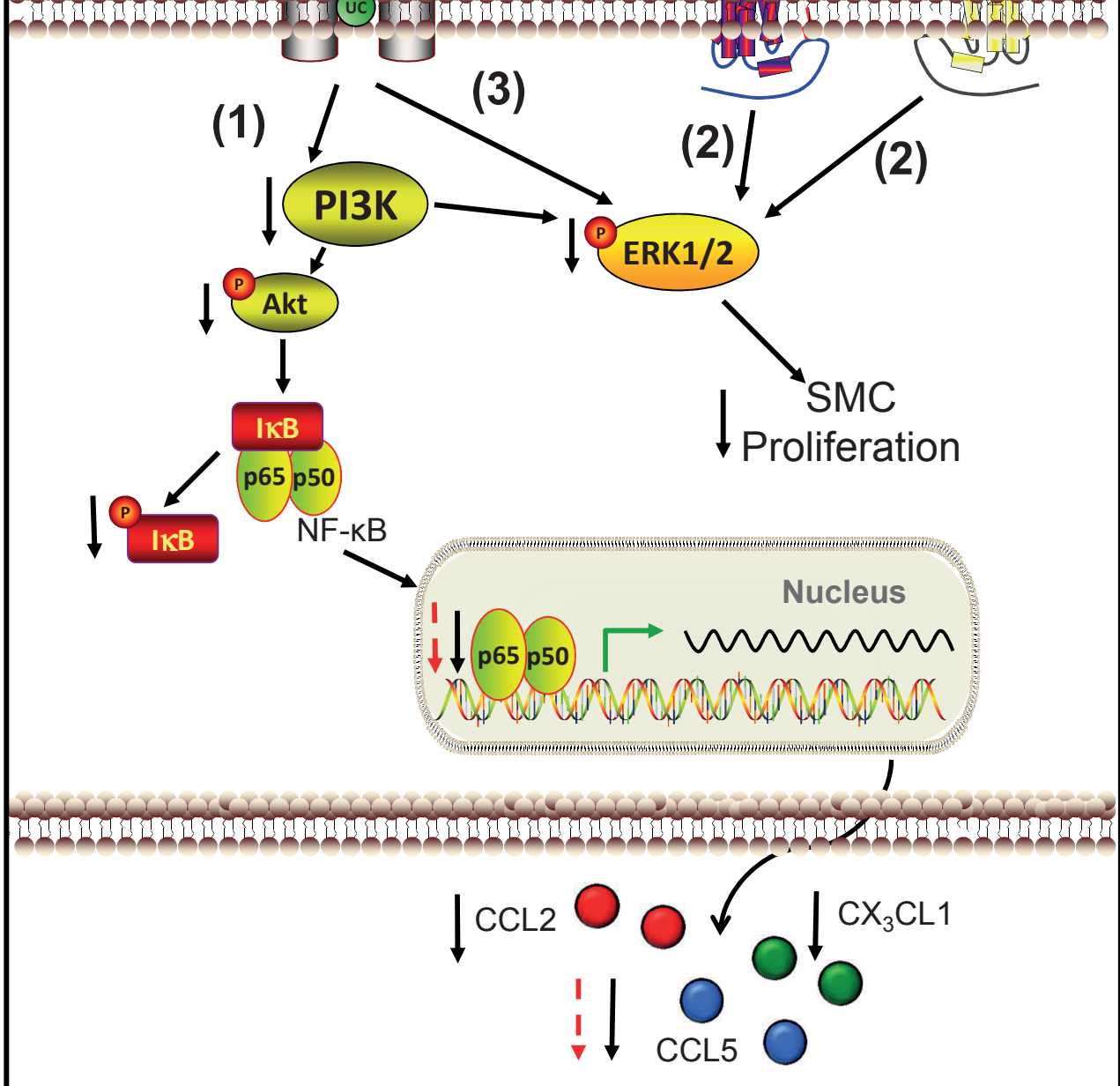


Figure 9. Proposed mechanisms for rHDL-induced inhibition of SMC chemokine expression and proliferation. Under inflammatory conditions rHDL suppress the chemokines CCL2, CCL5 and CX ${ }_{3}$ CL1 via SR-B1 and inhibit the PI3K/Akt//kB $\alpha / N F-\kappa B$ pathway (1). SMC proliferation is inhibited by rHDL via a reduction in chemokines CCL2, CCL5 and $\mathrm{CX}_{3} \mathrm{CL} 1$ and the chemokine receptors $\mathrm{CCR} 2$ and $\mathrm{CX}_{3} \mathrm{CR} 1$, indicating reduced chemokine/chemokine receptor interaction and a subsequent reduction in ERK phosphorylation (2). An additional pathway for suppression of proliferation is via SR-B1 and the inhibition of ERK phosphorylation (3). Cholesterol efflux, via SR-B1, partially mediates the inhibitory effects of rHDL on p65 and SMC chemokine expression (dashed red lines). 


\section{References}

1. Rye KA, Bursill CA, Lambert G, Tabet F, Barter PJ. The metabolism and antiatherogenic properties of hdl. Journal of lipid research. 2009;50 Suppl:S195-200

2. Barter PJ, Nicholls S, Rye KA, Anantharamaiah GM, Navab M, Fogelman AM. Antiinflammatory properties of hdl. Circulation research. 2004;95:764-772

3. Charo IF, Ransohoff RM. The many roles of chemokines and chemokine receptors in inflammation. The New England journal of medicine. 2006;354:610621

4. Chandrasekar B, Mummidi S, Perla RP, Bysani S, Dulin NO, Liu F, et al. Fractalkine (cx3cl1) stimulated by nuclear factor kappab (nf-kappab)-dependent inflammatory signals induces aortic smooth muscle cell proliferation through an autocrine pathway. The Biochemical journal. 2003;373:547-558

5. Kovacic JC, Gupta R, Lee AC, Ma M, Fang F, Tolbert CN, et al. Stat3-dependent acute rantes production in vascular smooth muscle cells modulates inflammation following arterial injury in mice. The Journal of clinical investigation. 2010;120:303-314

6. Schober A. Chemokines in vascular dysfunction and remodeling. Arteriosclerosis, thrombosis, and vascular biology. 2008;28:1950-1959

7. Selzman $\mathrm{CH}$, Miller SA, Zimmerman MA, Gamboni-Robertson F, Harken AH, Banerjee A. Monocyte chemotactic protein-1 directly induces human vascular smooth muscle proliferation. Am J Physiol Heart Circ Physiol. 2002;283:H14551461

8. White GE, Tan TC, John AE, Whatling C, McPheat WL, Greaves DR. Fractalkine has anti-apoptotic and proliferative effects on human vascular smooth muscle cells via epidermal growth factor receptor signalling. Cardiovasc Res. 2010;85:825-835

9. Furukawa $\mathrm{Y}$, Matsumori A, Ohashi N, Shioi T, Ono K, Harada A, et al. Antimonocyte chemoattractant protein $-1 /$ monocyte chemotactic and activating factor antibody inhibits neointimal hyperplasia in injured rat carotid arteries. Circulation research. 1999;84:306-314

10. Schober A, Zernecke A, Liehn EA, von Hundelshausen P, Knarren S, Kuziel WA, et al. Crucial role of the $\mathrm{ccl} 2 / \mathrm{ccr} 2$ axis in neointimal hyperplasia after arterial injury in hyperlipidemic mice involves early monocyte recruitment and ccl2 presentation on platelets. Circulation research. 2004;95:1125-1133

11. Taubman MB, Rollins BJ, Poon M, Marmur J, Green RS, Berk BC, et al. Je mrna accumulates rapidly in aortic injury and in platelet-derived growth factorstimulated vascular smooth muscle cells. Circulation research. 1992;70:314-325 
12. Zernecke A, Liehn EA, Gao JL, Kuziel WA, Murphy PM, Weber C. Deficiency in ccr5 but not ccr1 protects against neointima formation in atherosclerosisprone mice: Involvement of il-10. Blood. 2006;107:4240-4243

13. Roque M, Kim WJ, Gazdoin M, Malik A, Reis ED, Fallon JT, et al. Ccr2 deficiency decreases intimal hyperplasia after arterial injury. Arteriosclerosis, thrombosis, and vascular biology. 2002;22:554-559

14. Schepers A, Eefting D, Bonta PI, Grimbergen JM, de Vries MR, van Weel V, et al. Anti-mcp-1 gene therapy inhibits vascular smooth muscle cells proliferation and attenuates vein graft thickening both in vitro and in vivo. Arteriosclerosis, thrombosis, and vascular biology. 2006;26:2063-2069

15. Eefting D, Bot I, de Vries MR, Schepers A, van Bockel JH, Van Berkel TJ, et al. Local lentiviral short hairpin rna silencing of ccr2 inhibits vein graft thickening in hypercholesterolemic apolipoprotein e3-leiden mice. J Vasc Surg. 2009;50:152160

16. Rahmani M, Cruz RP, Granville DJ, McManus BM. Allograft vasculopathy versus atherosclerosis. Circulation research. 2006;99:801-815

17. Bursill CA, Castro ML, Beattie DT, Nakhla S, van der Vorst E, Heather AK, et al. High-density lipoproteins suppress chemokines and chemokine receptors in vitro and in vivo. Arteriosclerosis, thrombosis, and vascular biology. 2010;30:17731778

18. Di Bartolo BA, Nicholls SJ, Bao S, Rye KA, Heather AK, Barter PJ, et al. The apolipoprotein a-i mimetic peptide etc-642 exhibits anti-inflammatory properties that are comparable to high density lipoproteins. Atherosclerosis. 2011;217:395-400

19. Ibanez B, Giannarelli C, Cimmino G, Santos-Gallego CG, Alique M, Pinero A, et al. Recombinant hdl(milano) exerts greater anti-inflammatory and plaque stabilizing properties than hdl(wild-type). Atherosclerosis. 2012;220:72-77

20. Nicholls SJ, Dusting GJ, Cutri B, Bao S, Drummond GR, Rye KA, et al. Reconstituted high-density lipoproteins inhibit the acute pro-oxidant and proinflammatory vascular changes induced by a periarterial collar in normocholesterolemic rabbits. Circulation. 2005;111:1543-1550

21. Scanu A, Oliviero F, Gruaz L, Sfriso P, Pozzuoli A, Frezzato F, et al. Highdensity lipoproteins downregulate $\mathrm{ccl} 2$ production in human fibroblastlike synoviocytes stimulated by urate crystals. Arthritis research \& therapy. 2010;12:R23

22. Weisweiler P. Isolation and quantitation of apolipoproteins a-i and a-ii from human high-density lipoproteins by fast-protein liquid chromatography. Clin Chim Acta. 1987;169:249-254

23. Matz CE, Jonas A. Micellar complexes of human apolipoprotein a-i with 
phosphatidylcholines and cholesterol prepared from cholate-lipid dispersions. The Journal of biological chemistry. 1982;257:4535-4540

24. Takayama M, Itoh S, Nagasaki T, Tanimizu I. A new enzymatic method for determination of serum choline-containing phospholipids. Clin Chim Acta. 1977;79:93-98

25. McGrath KC, Li XH, Puranik R, Liong EC, Tan JT, Dy VM, et al. Role of 3betahydroxysteroid-delta 24 reductase in mediating antiinflammatory effects of high-density lipoproteins in endothelial cells. Arteriosclerosis, thrombosis, and vascular biology. 2009;29:877-882

26. Shimizu K, Minami M, Shubiki R, Lopez-llasaca M, MacFarlane L, Asami Y, et al. Cc chemokine receptor-1 activates intimal smooth muscle-like cells in graft arterial disease. Circulation. 2009;120:1800-1813

27. Topakian R, Sonnberger M, Nussbaumer K, Haring HP, Trenkler J, Aichner FT. Postprocedural high-density lipoprotein cholesterol predicts carotid stent patency at 1 year. European journal of neurology : the official journal of the European Federation of Neurological Societies. 2008;15:179-184

28. Seo SM, Choo EH, Koh YS, Park MW, Shin DI, Choi YS, et al. High-density lipoprotein cholesterol as a predictor of clinical outcomes in patients achieving low-density lipoprotein cholesterol targets with statins after percutaneous coronary intervention. Heart. 2011;97:1943-1950

29. Robbesyn F, Garcia V, Auge N, Vieira O, Frisach MF, Salvayre R, et al. Hdl counterbalance the proinflammatory effect of oxidized Idl by inhibiting intracellular reactive oxygen species rise, proteasome activation, and subsequent nf-kappab activation in smooth muscle cells. FASEB journal : official publication of the Federation of American Societies for Experimental Biology. 2003; $17: 743-745$

30. Mineo C, Shaul PW. Role of high-density lipoprotein and scavenger receptor $\mathbf{b}$ type $\mathbf{i}$ in the promotion of endothelial repair. Trends in cardiovascular medicine. 2007;17:156-161

31. Seetharam D, Mineo C, Gormley AK, Gibson LL, Vongpatanasin W, Chambliss $\mathrm{KL}$, et al. High-density lipoprotein promotes endothelial cell migration and reendothelialization via scavenger receptor-b type i. Circulation research. 2006;98:63-72

32. Feng $Y$, van Eck M, Van Craeyveld E, Jacobs F, Carlier V, Van Linthout S, et al. Critical role of scavenger receptor-bi-expressing bone marrow-derived endothelial progenitor cells in the attenuation of allograft vasculopathy after human apo a-i transfer. Blood. 2009;113:755-764

33. Murphy AJ, Hoang A, Aprico A, Sviridov D, Chin-Dusting J. Anti-inflammatory functions of apolipoprotein a-i and high-density lipoprotein are preserved 
in trimeric apolipoprotein a-i. The Journal of pharmacology and experimental therapeutics. 2013;344:41-49

34. Murphy AJ, Akhtari M, Tolani S, Pagler T, Bijl N, Kuo CL, et al. Apoe regulates hematopoietic stem cell proliferation, monocytosis, and monocyte accumulation in atherosclerotic lesions in mice. The Journal of clinical investigation. 2011;121:4138-4149

35. Gonzalez-Diez M, Rodriguez C, Badimon L, Martinez-Gonzalez J. Prostacyclin induction by high-density lipoprotein (hdl) in vascular smooth muscle cells depends on sphingosine 1-phosphate receptors: Effect of simvastatin. Thrombosis and haemostasis. 2008;100:119-126

36. Sviridov D, Fidge N, Beaumier-Gallon G, Fielding C. Apolipoprotein a-i stimulates the transport of intracellular cholesterol to cell-surface cholesterol-rich domains (caveolae). The Biochemical journal. 2001;358:79-86

37. Ashby DT, Rye KA, Clay MA, Vadas MA, Gamble JR, Barter PJ. Factors influencing the ability of hdl to inhibit expression of vascular cell adhesion molecule-1 in endothelial cells. Arteriosclerosis, thrombosis, and vascular biology. 1998;18:14501455

38. Baker PW, Rye KA, Gamble JR, Vadas MA, Barter PJ. Ability of reconstituted high density lipoproteins to inhibit cytokine-induced expression of vascular cell adhesion molecule-1 in human umbilical vein endothelial cells. Journal of lipid research. 1999;40:345-353

39. Saddar S, Carriere V, Lee WR, Tanigaki K, Yuhanna IS, Parathath S, et al. Scavenger receptor class $b$ type $\mathbf{i}$ is a plasma membrane cholesterol sensor. Circulation research. 2013;112:140-151 


\section{Acknowledgements}

This work was supported by a Heart Foundation Career Development Fellowship (CR07S3331) and a Bushell Foundation grant (to C.A.B), a National Health and Medical Research Council Program Grant (\#222722, to K-A. R), and a National Health and Medical Research Council Early Career Fellowship (\#537537, to L. L. D). 


\section{Supplemental Figures}

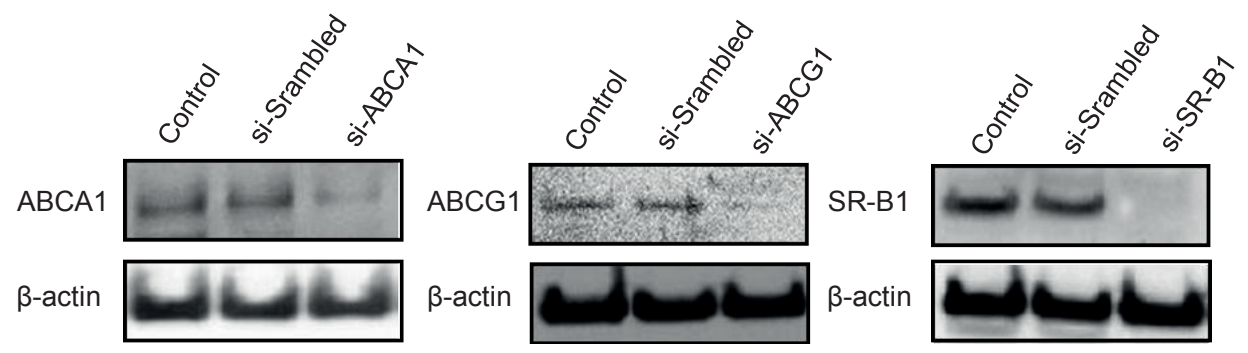

Supplemental Figure 1. siRNA knockdown of ABCA1, ABCG1 and SR-B1 in SMCs. HUVSMCs were transfected with scrambled (Scr), ABCA1, ABCG1 or SR-B1 siRNA. After $72 \mathrm{hrs}$, Western blotting was used to determine changes in ABCA1, ABCG1 or SR-B1 protein levels on cell lysates.

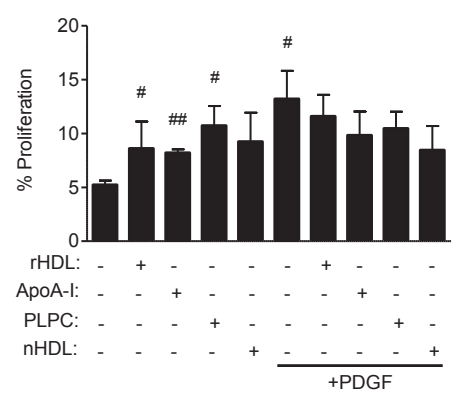

Supplemental Figure 2. rHDL have no effect on PDGF-stimulated SMC proliferation. HUVSMCs were serum starved by incubation with DMEM containing $0.1 \%$ Fetal Calf Serum (FCS, v/v) for $48 \mathrm{hrs}$. The cells were then incubated with rHDL, apoA-I, nHDL (all $600 \mu \mathrm{g} /$ $\mathrm{ml}$, final apoA-I concentration), phospholipid vesicles (PLPC, $2 \mu \mathrm{M}$ ) or PBS for 16 hrs prior to stimulation with PDGF-BB $(20 \mathrm{ng} / \mathrm{ml})$ and $\mathrm{EdU}(40 \mu \mathrm{M})$ for a further $48 \mathrm{hrs}$. The cells were then fixed and stained according to the "Click-IT" proliferation protocol. Results are presented as mean \pm SEM. ${ }^{*} p<0.05$, compared to non-treated control cells. 


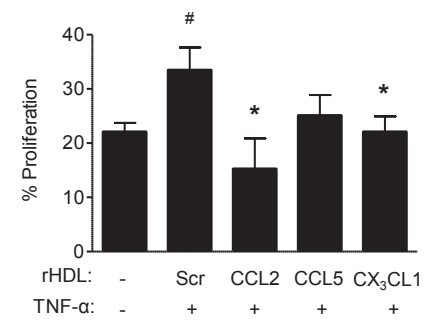

Supplemental Figure 3. The role of chemokines in SMC proliferation. HUVSMCs were serum starved by incubation with DMEM containing $0.1 \%$ Fetal Calf Serum (FCS, v/v) for 48 hrs and transfected with scrambled (Scr), CCL2, CCL5 and $\mathrm{CX}_{3} \mathrm{CL} 1$ siRNA prior to stimulation with TNF- $\alpha(1 \mathrm{ng} / \mathrm{ml})$ and EdU $(40 \mu \mathrm{M})$ for a further $48 \mathrm{hrs}$. The cells were then fixed and stained according to the "Click-IT" proliferation protocol. Results are presented as mean \pm SEM. ${ }^{*} p<0.05$, compared to non-treated control cells. ${ }^{* *} p<0.05$, compared to cells incubated with TNF- $\alpha$ only. 


\section{Chapter 7}

\section{High Density Lipoproteins exert pro- inflammatory effects on macrophages via passive cholesterol depletion and PKC-dependent NF-KB/STAT1 activation}

Emiel P.C. van der Vorst, Kosta Theodorou, Marten A. Hoeksema, Pieter Goossens, Christina A. Bursill, Sander W. Tas, Leonie Huitema, Ine M.J. Wolfs, Debby P.Y. Koonen, Shahla Abdollahi-Roodsaz, Toby Lawrence, Jogchum Plat, Miranda van Eck, Kerry-Anne Rye, Menno P.J. de Winther, Erik A.L. Biessen, Marjo M.P.C. Donners.

Cell Metabolism; In revision 


\section{Abstract}

Membrane cholesterol is known to modulate a variety of cell signaling pathways and functions. While cholesterol depletion by High-Density Lipoproteins (HDL) have potent anti-inflammatory effects in various celltypes, its effects on inflammatory responses in macrophages remain ill defined. Here we show overt pro-inflammatory effects of HDL mediated passive cholesterol depletion and lipid raft disruption in murine and human primary macrophages in vitro. These pro-inflammatory effects are confirmed in vivo in peritoneal macrophages from ApoA-I transgenic mice. Native and reconstituted HDL enhance Toll Like Receptor-induced signaling by activating an atypical PKC-NF-kB/STAT1 axis, leading to enhanced IL-12 and decreased IL-10 production. In parallel HDL enhance ADAM protease activity, thereby mediating TNF- $\alpha$ release. Such pro-inflammatory activities on macrophages could at least partly underlie the disappointing therapeutic potential of HDL raising therapy in cardiovascular clinical trials. 


\section{Introduction}

Innate immunity is the first line of defense against a variety of pathogens and tissue damage ${ }^{1}$. Macrophages are crucial actors in this process, employing a range of intra- and extracellular pattern recognition receptors (PRRs) to recognize foreign substances ${ }^{1}$. Key PRRs are the Toll-like receptors (TLRs), which upon ligand recognition activate several signaling pathways, thereby regulating immune cell functions including pro-inflammatory cytokine production, cell survival, apoptosis and phagocytosis ${ }^{2}$. Membrane cholesterol, more specifically lipid rafts play a pivotal role in the modulation of signaling, including TLR pathways, and subsequent cellular functions ${ }^{3}$. Indeed, cholesterol depletion by High Density Lipoproteins (HDL) is well known to exert anti-inflammatory, anti-oxidant, anti-thrombotic and anti-apoptotic functions ${ }^{4}$. Hence, plasma HDL levels have been inversely associated with clinical events resulting from atherosclerosis ${ }^{5}$, a chronic inflammatory disease of the vessel wall characterized by progressive accumulation of lipids, debris and inflammatory cells, predominantly macrophages. We have previously shown that HDL reduce the expression of pro-inflammatory chemokines in endothelial cells and smooth muscle cells in a Scavenger Receptor-B1 dependent manner, by inhibiting the canonical nuclear factor$\mathrm{KB}(\mathrm{NF}-\mathrm{KB})$ pathway, providing further insight into the mechanism of its antiinflammatory effects ${ }^{6,7}$.

In contrast to the protective effects in endothelial and smooth muscle cells, however, little is known about the effects of HDL on inflammatory cells. Only few studies investigated the effects of lipid-free ApoA-I ${ }^{8}$ or ApoA-I reconstituted with soy-bean phosphatidylcholine ${ }^{9}$ on macrophages, showing pro-inflammatory or anti-inflammatory effects, respectively. Although the transcription factor ATF3 has been implicated in HDL mediated responses in the latter study, the signaling pathways affected by HDL in macrophage inflammatory responses largely remain to be elucidated.

Here we report that both reconstituted and native HDL enhance TLR-induced pro-inflammatory responses in murine, both in vitro and in vivo, and in human macrophages. We propose a dual mechanism via cholesterol depletion and subsequent protein kinase $C$ activation leading to NF-KB/STAT1 dependent modulation of IL-10/IL-12 production as well as A Disintegrin And Metalloprotease (ADAM)-mediated release of TNF- $\alpha$. 


\section{Methods}

\section{Reagents}

Ultrapure LPS (E.coli 055:B5) was from Sigma. Polymyxin was obtained from Life Technologies. Pam ${ }_{3} \mathrm{CSK}_{4}(\mathrm{PAM})$, Poly:IC, R848 and CpG were from Invivogen. Methyl- $\beta$-cyclodextrin was from Sigma. $1 \mathrm{mg} / \mathrm{ml}$ Cholesterol / $4 \%$ (vol/vol) methyl- $\beta$-cyclodextrin mixture was prepared by vortexing at $37^{\circ} \mathrm{C}$ (50 $\mathrm{mg} / \mathrm{ml}$ stock solution of cholesterol in ethanol). Ro31-8425 and casein kinase II-inhibitor were from Merck Millipore. GI54023X and GW280264X were a kind gift from Glaxo Smith Kline. Enbrel was from Amgen.

\section{Preparation of native HDLs and discoidal reconstituted HDLs}

HDLs were isolated from human plasma by ultracentrifugation to isolate native HDLs (nHDLs) or commercially bought (cHDLs, GenWay Biotech). To prepare discoidal reconstituted HDLs (rHDLs) human plasma was ultracentrifuged, delipidated and subjected to anion-exchange chromatography using a Fast Protein Liquid Chromatography (FPLC) system to isolate ApoA-I ${ }^{10}$. Discoidal rHDL containing apoA-I complexed to 1-palmitoyl-2-linoleoylphosphatidylcholine (PLPC, Avanti Polar Lipids), initial PLPC/apoA-I molar ratio $100: 1$, were prepared by the cholate dialysis method ${ }^{11}$. The protein concentration of apoA-I was determined using the nanodrop (Thermo Scientific). Phospholipid concentrations were enzymatically determined (Wako) ${ }^{12}$. Limulus test was performed to exclude potential endotoxin contaminations (HyCult biotechnology).

\section{Animals}

All animal experiments were approved by the Animal Ethics Committee of the Maastricht University (permit numbers 2010-178 and 2012-090). All mice were 9-16 weeks old when used for experiments. Human ApoA-I transgenic mice, ABCA1, ABCG1, SR-B1, CD36, IKK ${ }^{\text {AAAA }}$, IKK $\beta$, NIK, STAT1 (generously donated by Dr. Mathieu and Dr. Gysemans) deficient mice and NF-KB Luciferase mice were all on a C57BI/6 background. Age- and gender matched $\mathrm{C} 57 \mathrm{BI} / 6$ control mice were purchased from Charles River Laboratories. 


\section{Cell culture and treatments}

Human Coronary Artery Endothelial Cells (HCAECs; Lonza) were cultured using EGM-2 MV BulletKit (Lonza) and grown until 90\% confluent. Cells were incubated with HDLs for $24 \mathrm{hrs}$, washed and stimulated with $2.5 \mathrm{ng} / \mathrm{ml}$ TNF- $\alpha$ (R\&D systems) for 5 hrs.

Bone marrow was isolated from femurs and tibiae of described mice. Cells were cultured in RPMI-1640 medium (GIBCO Invitrogen) supplemented with 10\% (vol/vol) heat inactivated fetal calf serum (Bodinco B.V.), $100 \mathrm{U} / \mathrm{ml}$ penicillin, $100 \mu \mathrm{g} / \mathrm{ml}$ streptomycin, $2 \mathrm{mM}$ L-glutamine (All GIBCO Invitrogen) and $20 \%$ ( $\mathrm{vol} / \mathrm{vol}$ ) L929-conditioned medium (LCM) for 8-9 days to differentiate into bone marrow-derived macrophages (BMDMs). Macrophages were incubated with various concentrations of HDLs for $24 \mathrm{hrs}$, washed and stimulated for 6 or $24 \mathrm{hrs}$ with $0-10 \mathrm{ng} / \mathrm{ml}$ LPS.

Resident peritoneal macrophages were obtained by flushing the peritoneal cavity with ice-cold PBS followed by culturing in RPMI 1640 culture medium containing $10 \%$ ( $\mathrm{vol} / \mathrm{vol}) \mathrm{FCS}$, penicillin $(100 \mathrm{U} / \mathrm{ml})$, streptomycin $(100 \mathrm{ug} / \mathrm{ml})$, and L-glutamine $2 \mathrm{mM}$ (all GIBCO Invitrogen). After overnight attachment, floating cells were removed while attached cells were incubated with various concentrations of HDLs for $24 \mathrm{hrs}$, washed and stimulated for 6 or $24 \mathrm{hrs}$ with 0-10 ng/ml LPS.

Human monocyte derived macrophages were obtained by isolating mononuclear cells from healthy donor buffy coats (Sanquin) using Lymphoprep (AXIS-SHIELD) density gradient centrifugation. Monocytes were further isolated by Standard Isotone Percoll gradient centrifugation (Amersham) and plated in Iscove modified Dulbecco medium (IMDM, Life Tech), supplemented with $1 \%$ (vol/vol) fetal bovine serum (FBS, Life Tech) for $30 \mathrm{~min}$ at $37^{\circ} \mathrm{C}$. Non-adherent cells were removed, and monocytes cultured for 7 days in IMDM containing 10\% (vol/vol) FBS, 1\% (vol/vol) penicillin/ streptomycin (Life Tech) and $10 \mathrm{ng} / \mathrm{ml}$ M-CSF (Miltenyi). Macrophages were incubated with various concentrations of HDLs for $24 \mathrm{hrs}$, washed and stimulated for 6 or $24 \mathrm{hrs}$ with $0-10 \mathrm{ng} / \mathrm{ml}$ LPS. 


\section{Gene expression}

RNA was isolated using the High Pure RNA Isolation Kit (Roche), according to manufacturer's instructions, after which RNA content was quantified using the Nanodrop. Total RNA was normalized and reverse transcribed using iScript (BioRad). Quantitative PCR was performed using $10 \mathrm{ng}$ cDNA, 300 $\mathrm{nM}$ of each primer, and SensiMix (Quantace-Bioline). All gene expression levels were corrected for cyclophilin $A$ and $\beta$-actin as housekeeping genes. Primer sequences are available upon request.

\section{Enzyme-Linked Immunosorbent Assay (ELISA)}

IL-12 and TNF- $\alpha$ ELISA assays(Invitrogen) and IL-10 ELISA assay (eBioscience) were performed on conditioned medium according to manufacturer's instructions. Analysis was performed using a micro-plate reader (Bio-Rad) at $450 \mathrm{~nm}$.

\section{NO assay}

NO production was measured in conditioned medium using Griess reagent (2.5\% (vol/vol) $\mathrm{H}_{3} \mathrm{PO}_{4}, 1 \%$ (wt/vol) sulfanilamide, $0.1 \%$ (wt/vol) naphthalene diamine dihydrochloride). Analysis was performed using a micro-plate reader (Bio-Rad) at $550 \mathrm{~nm}$.

\section{Cholesterol efflux assay}

BMDMs were incubated for $24 \mathrm{hrs}$ with ${ }^{3} \mathrm{H}$ Cholesterol. Cells were washed and equilibrated for $1 \mathrm{hrs}$. After equilibration cells are incubated for $24 \mathrm{hrs}$ with $300 \mathrm{ug} / \mathrm{ml} \mathrm{nHDL}$ or $\mathrm{rHDL}$, after which radioactivity was measured in the media and cell lysates.

\section{Flow cytometry analysis}

C57BI/6 and ApoA-I transgenic mice were intra-peritoneally stimulated with $20 \mu \mathrm{g}$ LPS for $48 \mathrm{hrs}$ or with $10 \mu \mathrm{g}$ R848 for 2 hrs prior to isolation. Resident peritoneal macrophages were obtained by flushing the peritoneal cavity with ice-cold PBS followed by culturing in RPMI 1640 culture medium containing $10 \%$ ( $\mathrm{vol} / \mathrm{vol}) \mathrm{FCS}$, penicillin $(100 \mathrm{U} / \mathrm{ml})$, streptomycin $(100 \mathrm{ug} / \mathrm{ml})$, and L-glutamine $2 \mathrm{mM}$ (all GIBCO Invitrogen). After overnight attachment, floating cells were removed while attached cells were activated by $10 \mathrm{ng} / \mathrm{ml}$ LPS or $10 \mu \mathrm{g} / \mathrm{ml}$ R848 in combination with GolgiSTOP (BD) for $9 \mathrm{hrs}$. Cells were subsequently collected and stained according to described protocol ${ }^{13}$. All flow cytometry measurements were performed by a FACS CANTO II (BD) followed by data analysis using FACSdiva software. 


\section{Cholera toxin staining}

For lipid raft staining, bone marrow-derived macrophages were plated on Lab-Tek borosilicate 8-chambered coverglasses (Thermo Fisher Scientific, Nunc) at a density of $2 \times 10^{5}$ cells/well. Once adhered, the macrophages were treated overnight with native HDL $(300 \mu \mathrm{g} / \mathrm{ml})$, reconstituted HDL $(300 \mu \mathrm{g} /$ $\mathrm{ml})$ or cyclodextrin $(4 \%(\mathrm{vol} / \mathrm{vol}))$ or cholesterol-loaded cyclodextrin $(4 \%$ $(\mathrm{vol} / \mathrm{vol}))$. Staining was performed using the Vybrant ${ }^{\circledR}$ Lipid Raft Labeling kit (Life Technologies, Molecular Probes) according to the manufacturer's protocol. In short, cells were washed with cold, serum-free DMEM and incubated with Alexa488-conjugated cholera toxin subunit B (CT-B) for 10 min at $4^{\circ} \mathrm{C}$, followed by lipid raft crosslinking with an anti-CT-B antibody for $15 \mathrm{~min}$ at $4^{\circ} \mathrm{C}$. Subsequently, the cells were fixed with $4 \%$ (vol/vol) Antigenfix (DiaPath) for $10 \mathrm{~min}$ on ice and nuclei were stained with TO-PRO-3. Pictures were made using an Axiovert LSM 510 confocal microscope (Zeiss) at a $63 x$ magnification. Total cell fluorescence was measured for each cell within six fields of view per treatment using the ImageJ software and corrected for background fluorescence (corrected total cell fluorescence $=$ total cell fluorescence - (average background fluorescence $x$ total cell area)). Data shown are representative for three independent experiments.

\section{NF-kB Luciferase assay}

BMDMs from NF-KB Luciferase reporter mice were lysed in luciferase lysis buffer. Luciferase activity was measured in supernatants of the lysates, according to the manufacturer's protocol (Promega), using a GloMax ${ }^{\circledR}-96$ Luminometer (Turner Biosystems).

\section{Western blotting}

BMDMs from C57BI/6 mice were incubated with $300 \mu \mathrm{g} / \mathrm{ml} \mathrm{nHDL}$ for $24 \mathrm{hrs}$, washed and stimulated for $5 \mathrm{~min}$ with $10 \mathrm{ng} / \mathrm{ml}$ LPS. Cells were washed with PBS and subsequently lysed and homogenized in equal volumes of lysis buffer (1\% (vol/vol) Triton, protease inhibitor, PhosSTOP, $150 \mathrm{mM} \mathrm{NaCl}, 200$ $\mathrm{mM}$ Tris and glycerol). Equal amounts of protein were separated on a $8 \%$ ( $\mathrm{vol} / \mathrm{vol}$ ) SDS-PAGE gel by electrophoresis and transferred to a nitrocellulose membrane. Membranes were probed with a primary antibody to phopho-NFKB p65 (1:1,000, Cell Signaling) followed by a peroxidase labeled secondary antibody to rabbit IgG (1:1,000, Cell Signaling). Phospho-p65 levels were normalized using $\beta$-actin (primary antibody to $\beta$-actin, 1:1,000, Cell signaling). Chemiluminescence was detected using a digital scanner. 


\section{Immunofluorescence staining}

BMDMs from C57BI/6 mice were plated on Lab-tek chamber slides (Thermo Scientific) and incubated with $300 \mu \mathrm{g} / \mathrm{ml} \mathrm{nHDL}$ for $24 \mathrm{hrs}$, washed and stimulated for $1 \mathrm{hr}$ with $0-10 \mathrm{ng} / \mathrm{ml}$ LPS. Cells were washed with PBS, fixed in ice cold aceton for $10 \mathrm{~min}$ and stored at $-20^{\circ} \mathrm{C}$. Defrosted slides were washed three times with PBS and incubated with a NF-kB p65-specific antibody (C20, Santa Cruz) for $1 \mathrm{hr}$ in $1 \%$ (wt/vol) bovine serum albumin (BSA) in PBS at room temperature. Slides were again washed 3 times with PBS and an Alexa Fluor 488 labeled antibody to rabbit IgG (A21206, Invitrogen) was diluted in $1 \%$ BSA, $10 \%$ ( $\mathrm{vol} / \mathrm{vol})$ donkey serum in PBS. Incubation with secondary antibody was $30 \mathrm{~min}$ at room temperature after which slides were washes 3 times with PBS. Finally, the slides were mounted with prolong ${ }^{\circledR}$ gold antifade moutant with DAPI (Invitrogen). As a negative control, sections were incubated with isotype controls (IgG rabbit, Santa Cruz) or PBS. The slides were analyzed using a Leica TCS SP8 X Confocal Microscope (Leica) using a 40x objective with digital zoom. On average 10 z-stacks spanning $\sim 1 \mu \mathrm{m}$ were captured and then flattened by maximum projection using the Leica software.

\section{Statistical analysis}

Data are presented as mean \pm the standard error of the mean (SEM). All statistical analyses were performed using the Prism program (GraphPad Software Inc). All data are from at least three independent experiments, unless stated otherwise. The statistical significance of differences was evaluated with the Student's t-test. Significance was accepted at the level of $p<0.05 .{ }^{*} p<0.05,{ }^{* *} p<0.01,{ }^{* * *} p<0.001$ or ${ }^{*} p<0.05,{ }^{\#} p<0.01,{ }^{\# \#} p<0.001$. 


\section{Results}

\section{HDL enhance inflammation in murine macrophages}

In keeping with previous studies ${ }^{6,14}$, HDL treatment of endothelial cells significantly reduced TNF- $\alpha$ elicited gene expression of chemotactic and adhesion molecules CCL2, CCL5, VCAM-1 and E-selectin in human coronary artery endothelial cells (HCAECs, Fig. 1A). These data confirm that the HDL preparations exert anti-inflammatory effects on endothelial cells as demonstrated before.

Next we studied effects of HDL on inflammatory responses on bone marrow derived macrophages (BMDMs) from C57BI6 mice. HDL functionality in BMDMs was firmly established by a macrophage cholesterol efflux assay, showing $53 \%$ efflux capacity of 0-300 $\mu \mathrm{g} / \mathrm{ml}$ discoidal reconstituted $\mathrm{HDL}$ (rHDL, consisting of merely ApoA-I and 1-palmitoyl-2-linoleoyl-phosphatidylcholine (PLPC)) and 63\% efflux capacity of native HDL (nHDL) (Fig. 1B), as previously published ${ }^{15}$. Much to our surprise, treatment of BMDMs with rHDL resulted in a significant, concentration dependent increased gene expression of proinflammatory IL-12 and TNF- $\alpha$ (Fig. 1C), whereas anti-inflammatory IL-10 was significantly decreased (Fig. 1C). Additionally, at protein level rHDL treated BMDMs had augmented LPS-induced pro-inflammatory cytokine secretion (Fig. 1D), whereas IL-10 secretion was significantly reduced (Fig. 1D). To verify whether these effects are limited to reconstituted/semi-synthetic rHDL, we tested mature $\mathrm{nHDL}$ preparations isolated in our lab or obtained through commercial sources (cHDL). Both lipoprotein batches showed comparable pro-inflammatory effects on LPS-induced cytokine secretion by BMDMs (Fig. $1 E-F)$, illustrating the broad validity of our findings for reconstituted and blood derived HDL. Although, commercial HDL does not seem to influence TNF- $\alpha$ secretion, indicating some specificity in HDL subtypes. All HDL preps were negative for endotoxin as tested in a Limulus test (i.e., $<0.01 \mathrm{EU}[<1 \mathrm{pg}]$

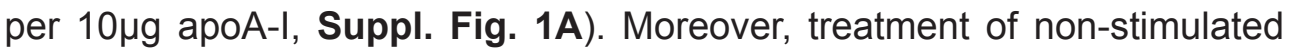
BMDMs with HDL failed to induce inflammatory responses, while as a positive reference LPS already showed drastic responses at very low concentrations (Suppl. Fig. 1B). Finally, BMDMs treated with HDL in the presence of the endotoxin neutralizing agent polymyxin $B$, displayed similar pro-inflammatory effects (Suppl. Fig. 1C). These data exclude that endotoxin contaminations of the HDLs preparations are responsible for HDL's pro-inflammatory activity. 
Together our data demonstrate clear pro-inflammatory effects of various functional, endotoxin-free HDL preps on mouse BMDMs, while exerting antiinflammatory effects on HCAECs as published before ${ }^{6,14}$.

A
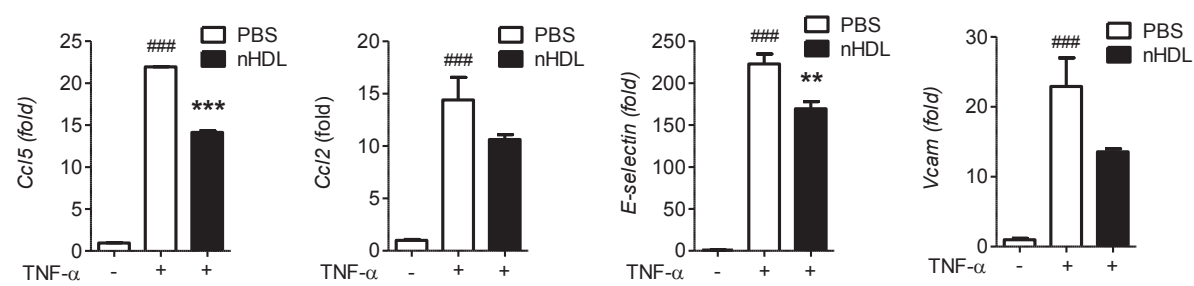

B

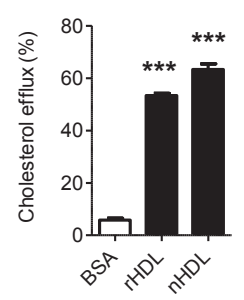

C
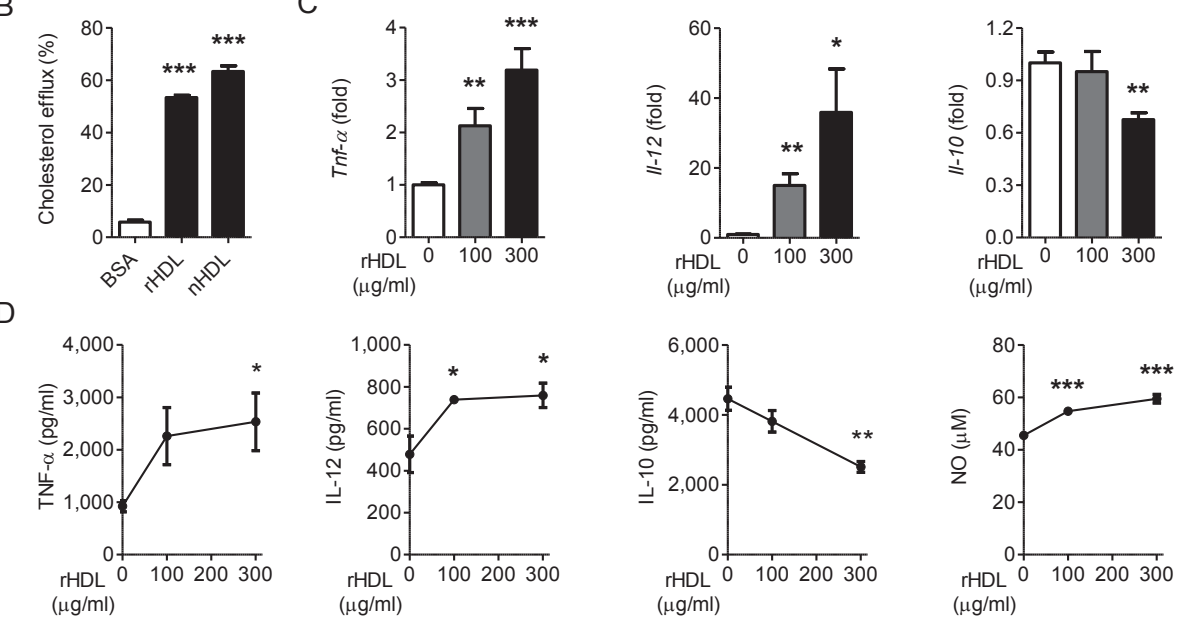

$E$
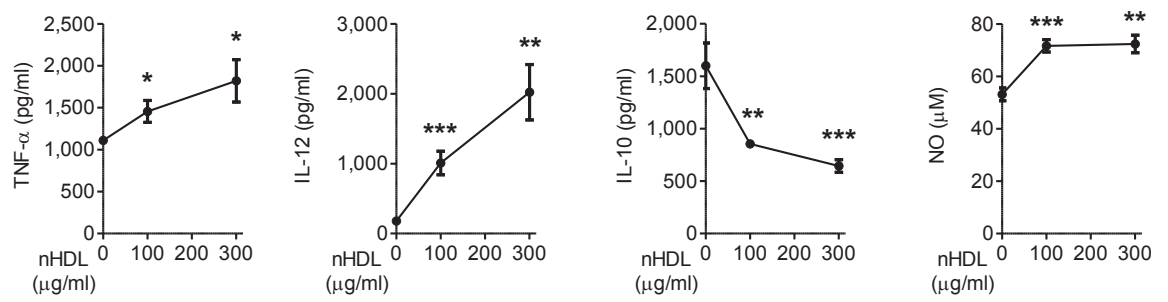

F
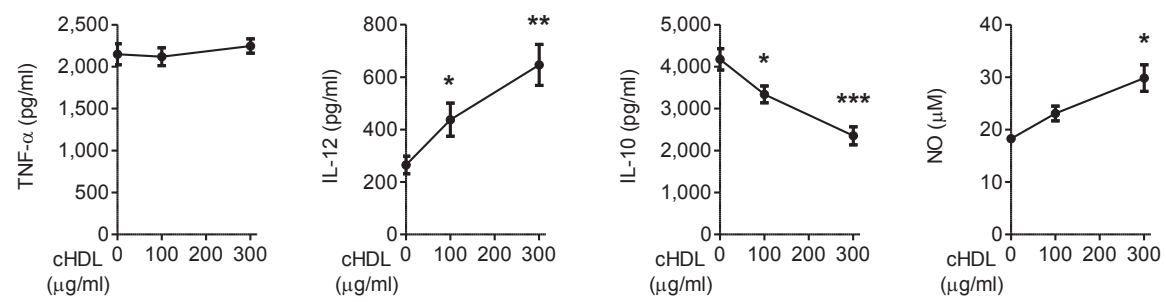
Figure 1. HDL increase inflammatory cytokine expression in mouse bone marrow derived macrophages (BMDMs). (A) mRNA expression in HCAECs pre-treated with $\mathrm{nHDL}$ $(600 \mu \mathrm{g} / \mathrm{ml})$ for $24 \mathrm{hrs}$ and stimulated with TNF- $\alpha(2.5 \mathrm{ng} / \mathrm{ml})$ for $5 \mathrm{hrs}$ quantified by PCR (representative figure of $n=3$ ). (B) Cholesterol efflux of ${ }^{3} \mathrm{H}$ cholesterol loaded BMDMs $(n=6)$ after treatment with $0.2 \%$ (wt/vol) BSA or $300 \mu \mathrm{g} / \mathrm{ml} \mathrm{HDL}$. Radioactivity was measured in the media and cell lysates and expressed as percentage of ${ }^{3} \mathrm{H}$ Cholesterol present in the media. (C) mRNA expression in BMDMs $(n=3)$ incubated for $24 \mathrm{hrs}$ with HDL. (D-F) ELISA of cytokines and Griess assay of NO secretion by BMDMs ( $n=3)$, pre-incubated with HDL for $24 \mathrm{hrs}$, washed and stimulated with $10 \mathrm{ng} / \mathrm{ml}$ LPS for $6 \mathrm{hrs}$ (TNF- $\alpha$ and IL-10) or 24 hrs (IL-12 and NO). BMDMs were either pre-incubated with rHDL (C-D), self-isolated $\mathrm{nHDL}$ (E) or commercially obtained $\mathrm{nHDL}(\mathrm{cHDL})(\mathbf{F})$. All results are expressed as mean \pm SEM. ${ }^{*} p<0.05,{ }^{* *} p<0.01,{ }^{* * *} p<0.001$, compared to non-HDL pre-incubated control cells. ${ }^{\# \#} p<0.001$, compared to non-TNF- $\alpha$ treated cells.

\section{HDL augment TLR-induced inflammation in macrophages in vivo}

The pro-inflammatory activity of HDL in BMDMs could be confirmed in murine primary peritoneal macrophages. In vitro HDL treatment of resident peritoneal macrophages resulted in comparable pro-inflammatory effects (Fig. 2A). Furthermore, HDL exerted similar pro-inflammatory responses on human peripheral blood monocyte-derived macrophages. Both rHDL and $\mathrm{nHDL}$ showed a concentration-dependent increment of LPS-induced pro-inflammatory TNF- $\alpha$ and IL-12 cytokine secretion, while that of antiinflammatory IL-10 was significantly decreased (Fig. 2B).

To corroborate whether these pro-inflammatory effects of HDLon macrophages also manifest in vivo, we examined transgenic mice, overexpressing human ApoA-I in the liver, resulting in increased circulating HDL levels ${ }^{16}$. Indeed, in vivo LPS activated peritoneal macrophages isolated from ApoA-I transgenic mice showed significantly increased pro-inflammatory IL-12 and TNF- $\alpha$ production compared to wildtype mice (Fig. 2C). Interestingly, the $\mathrm{HDL}$ associated increase in pro-inflammatory responses of BMDMs was not restricted to TLR4 stimulation by LPS, but was observed for TLR1/2, TLR3, TLR7/8 and TLR9 as well, though HDL's impact on TLR3 and TLR9 responses did not reach significance (Fig. 2D). Likewise, HDL enhanced also in vivo TLR7/8 responses, since R848 (TLR7/8 ligand) activated peritoneal macrophages isolated from ApoA-I transgenic mice showed a trend towards increased IL-12 and TNF- $\alpha$ production, compared to wildtype macrophages (Fig. 2E). Collectively, these data show that HDL exert TLR-induced proinflammatory effects in human and murine macrophages in vitro and in vivo. 


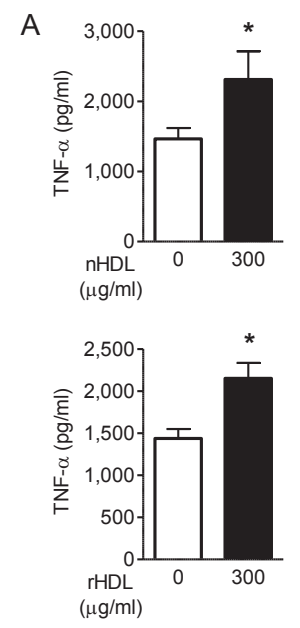

C

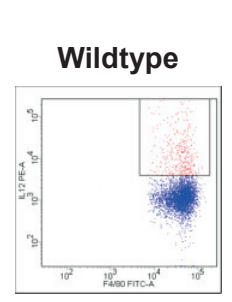

D

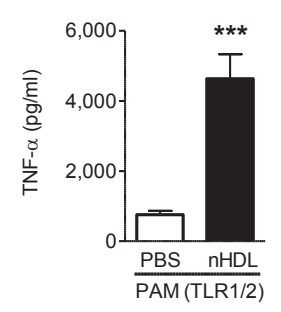

E

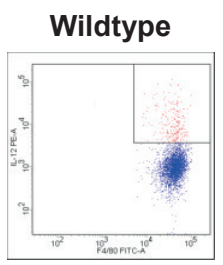

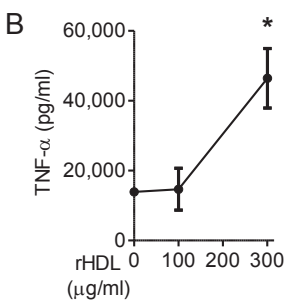

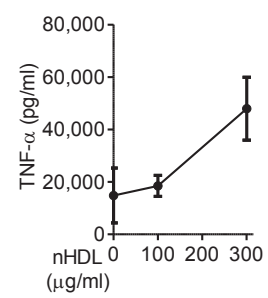

ApoA-I transgenic
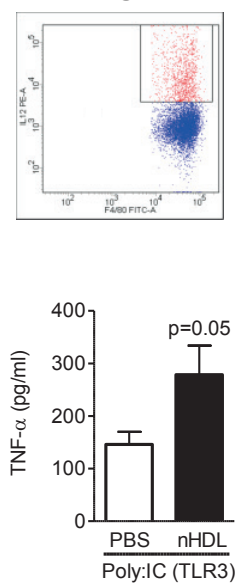

ApoA-I transgenic

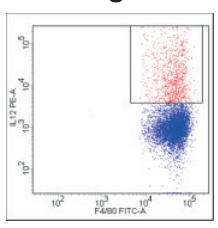

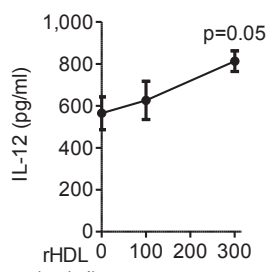
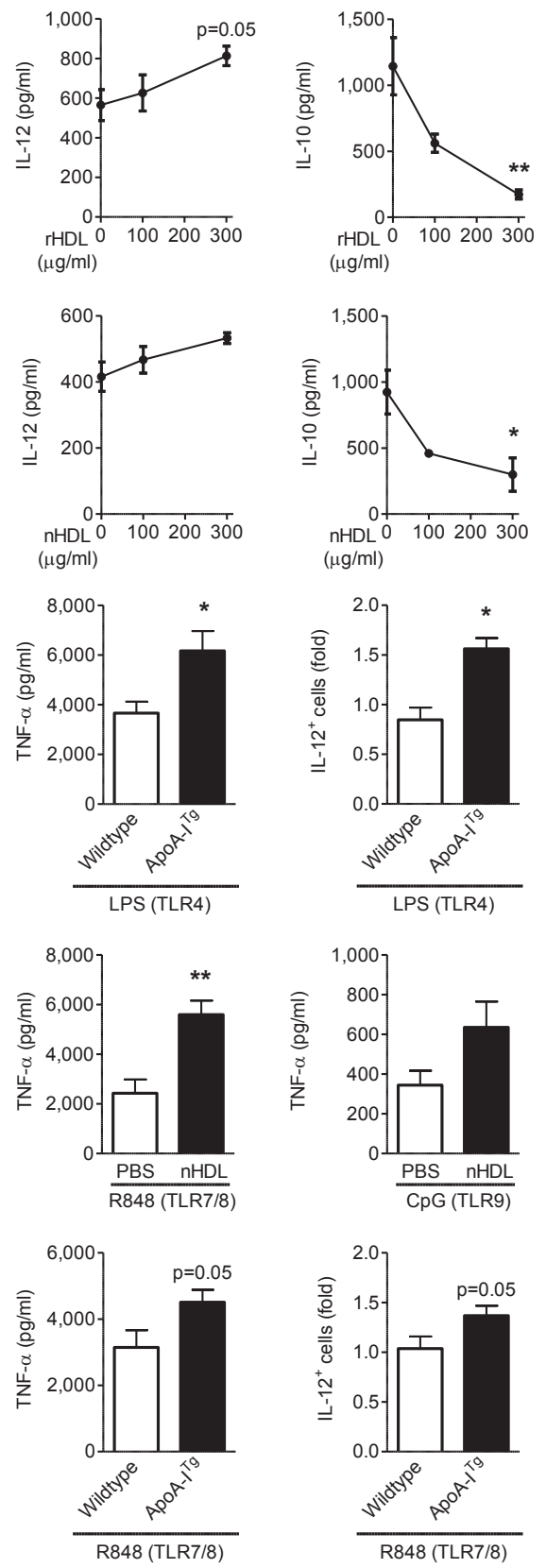
Figure 2. Pro-inflammatory effects of HDL on murine and human macrophages in vitro and in vivo. (A) TNF- $\alpha$ secretion from resident peritoneal macrophages from C57BI/6 mice $(n=3)$ was measured by ELISA after treatment with $\mathrm{rHDL}$ and $\mathrm{nHDL}(0-300 \mu \mathrm{g} / \mathrm{ml})$ for 24 hrs, washing and stimulation with LPS $(10 \mathrm{ng} / \mathrm{ml})$ for 6 hrs. (B) Peripheral blood monocyte derived human macrophages $(\mathrm{n}=3)$ pre-incubated with $\mathrm{rHDL}$ and $\mathrm{nHDL}(0-300 \mu \mathrm{g} / \mathrm{ml})$ for 24 hrs, washed and stimulated with $10 \mathrm{ng} / \mathrm{ml}$ LPS for 6 hrs (TNF- $\alpha$ and IL-10) or 24 hrs (IL12). Cytokine secretion was quantified using ELISA. (C) Representative intracellular FACS plots and quantification of IL-12+ peritoneal macrophages isolated from wildtype and ApoA-I transgenic mice $(n=6)$ after i.p. injection with $20 \mu \mathrm{g}$ LPS for $48 \mathrm{hrs}$. Ex vivo TNF- $\alpha$ secretion from isolated peritoneal macrophages was measured by ELISA after 6 hrs LPS stimulation. (D) TNF- $\alpha$ secretion of BMDMs $(n=3)$ stimulated with various TLR ligands quantified by ELISA. BMDMs were pre-incubated with $\mathrm{nHDL}(300 \mu \mathrm{g} / \mathrm{ml})$, washed and stimulated with 10 $\mu \mathrm{g} / \mathrm{ml} \mathrm{Pam}$ CSK $_{4}$ (TLR1/2), $10 \mu \mathrm{g} / \mathrm{ml}$ Poly:IC (TLR3), $10 \mu \mathrm{g} / \mathrm{ml} \mathrm{R848} \mathrm{(TLR7/8)} \mathrm{or} 5 \mathrm{ng} / \mathrm{ml}$ CpG (TLR9) for 6 hrs. (E) Representative intracellular FACS plots and quantification of IL-12+ peritoneal macrophages isolated from wildtype and ApoA-I transgenic mice $(n=6)$ after i.p. injection with $10 \mu \mathrm{g} 848$ for $2 \mathrm{hrs}$. TNF- $\alpha$ secretion from isolated peritoneal macrophages after 6 hrs R848 stimulation in ex vivo culture was quantified using ELISA. All results are expressed as mean \pm SEM. ${ }^{*} p<0.05,{ }^{* *} p<0.01$, ${ }^{* * *} p<0.001$, compared to non-treated control or wildtype cells. 


\section{Passive cholesterol efflux mediates pro-inflammatory HDL effects}

The cardioprotective activity of HDL has mainly been attributed to its capacity to enhance cholesterol efflux mediated by ABCA1, ABCG1, SR-B1 or CD36 17-19. Therefore we determined whether these transporters or scavenger receptors are implicated in HDL's pro-inflammatory activity using BMDMs isolated from the corresponding knockout mice. As shown before, analyses of wildtype BMDMs showed significant, concentration-dependent proinflammatory effects upon nHDL treatment (Fig. 3A). Remarkably, ABCA1, ABCG1, SR-B1 or CD36 deficiency was unable to prevent the HDL-induced increase in LPS response (Fig. 3A). BMDMs from these knockout mice even showed exacerbated IL-12 responses, as was the TNF- $\alpha$ response in SR-B1 knockouts. These data indicate that active cholesterol efflux by the aforementioned transporters or receptors is not responsible for HDL's proinflammatory activity in macrophages.

Besides active cholesterol efflux, HDL can also extract cholesterol from the cell membrane by passive diffusion. The latter has been shown to disrupt lipid rafts, which are cholesterol-rich domains important for signaling ${ }^{3}$. Cholera toxin-B (which binds the ganglioside GM-1, a marker for lipid rafts) was used to visualize and quantify membrane lipid rafts in BMDMs (Fig. 3B). $\mathrm{HDL}$ appeared to be equally effective in extracting membrane cholesterol by $40-50 \%$ as methyl- $\beta$-cyclodextrin, a known cholesterol depleting and lipid raft disrupting agent, reaffirming its functionality as cholesterol acceptor. Interestingly, treatment of BMDMs with methyl- $\beta$-cyclodextrin showed similar effects on LPS induced secretion of TNF- $\alpha$ and IL-10 by BMDMs than HDL, although it did not affect IL-12 (Fig. 3C), suggesting that the observed inflammatory response is at least partly associated with cholesterol extraction. Indeed treatment of BMDMs with methyl- $\beta$-cyclodextrin ablated the stimulatory effects of HDL on TNF- $\alpha$ and IL-10 secretion (Fig. $3 C$ ). In contrast, treatment of BMDMs with cholesterol saturated methyl- $\beta$-cyclodextrin failed to quench the pro-inflammatory effects of HDL (Fig. 3C). These results indicate that lipid rafts can play an important role in the observed pro-inflammatory effects. 

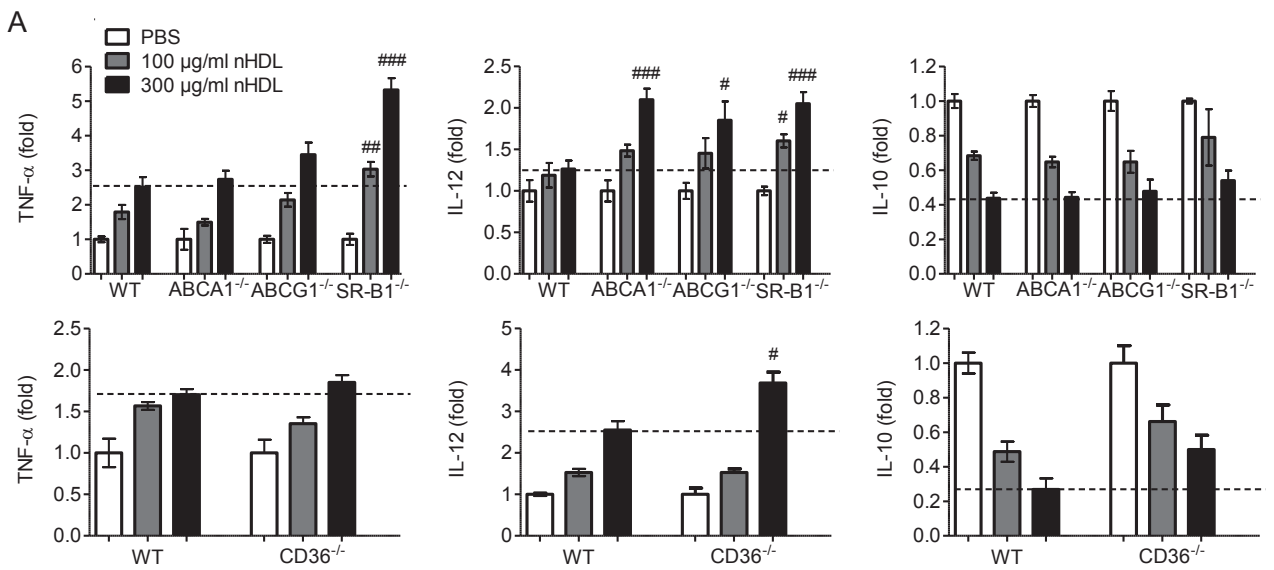

B
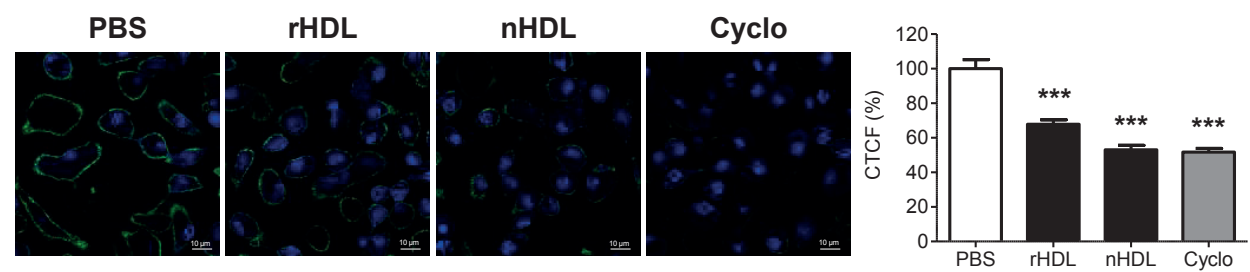

C

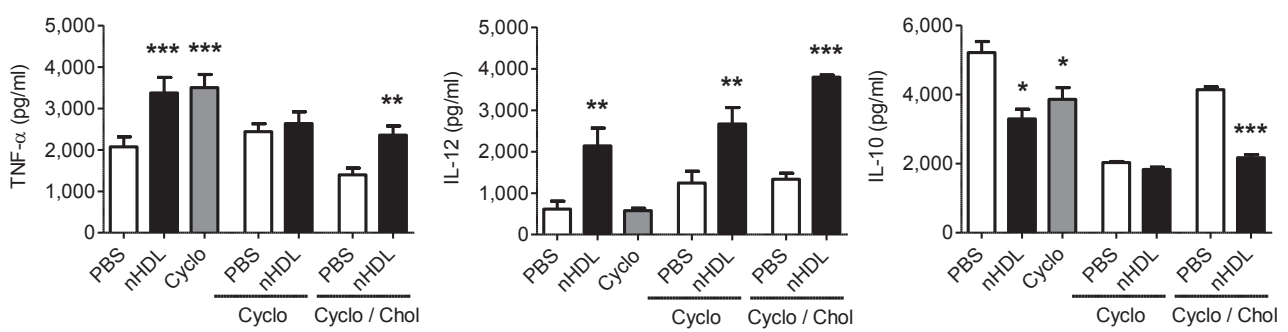


Figure 3. Lipid raft disruption by cholesterol depletion is responsible for pro-inflammatory

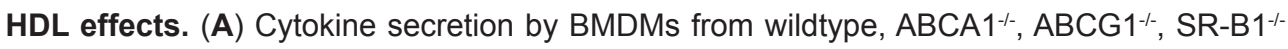
or $\mathrm{CD}^{-/-}$mice $(\mathrm{n}=3)$ pre-incubated with native $\mathrm{HDL}$, washed and stimulated with $10 \mathrm{ng} /$ ml LPS for 6 hrs (TNF- $\alpha$ and IL-10) or 24 hrs (IL-12). (B) BMDMs from C57Bl6 mice treated overnight with $300 \mu \mathrm{g} / \mathrm{ml} \mathrm{HDL}$ or $4 \mathrm{hrs}$ with $4 \%$ (vol/vol) methyl- $\beta$-cyclodextrin and stained for lipid rafts using cholera-toxin staining of the ganglioside GM-1. Representative pictures and quantification of three independent experiments are shown. (C) Cytokine secretion by BMDMs $(n=3)$ after cholesterol depletion by $24 \mathrm{hrs}$ treatment with $300 \mu \mathrm{g} / \mathrm{ml} \mathrm{nHDL}$ or $4 \mathrm{hrs}$ with $4 \%$ (vol/vol) methyl- $\beta$-cyclodextrin. BMDMs are pre-incubated for $24 \mathrm{hrs}$ with $300 \mu \mathrm{g} / \mathrm{ml}$ $\mathrm{nHDL}$ or $4 \mathrm{hrs}$ with $4 \%$ (vol/vol) cyclodextrin. Additionally, BMDMs were pre-treated for $4 \mathrm{hrs}$ with $4 \%(\mathrm{vol} / \mathrm{vol})$ cyclodextrin or cyclodextrin/cholesterol mixture, followed by treatment for 24 hrs with $300 \mu \mathrm{g} / \mathrm{ml}$ native HDL. After washing, BMDMs were stimulated with $10 \mathrm{ng} / \mathrm{ml}$ LPS for $6 \mathrm{hrs}$ (TNF- $\alpha$ and IL-10) or $24 \mathrm{hrs}$ (IL-12) and cytokine secretion was measured by ELISA. All results are expressed as mean \pm SEM. ${ }^{*} p<0.05,{ }^{* *} p<0.01,{ }^{* * *} p<0.001$, compared to nontreated control cells. ${ }^{\#} p<0.05,{ }^{\#} p<0.01,{ }^{\#} p<0.001$, compared to wildtype cells. 


\section{Pro-inflammatory HDL effects are mediated by NF-KB activation}

Lipid rafts harbor several receptors and signaling kinases, the activity of which will be impacted upon cholesterol depletion and subsequent lipid raft disruption ${ }^{20}$. Conceivably, passive HDL mediated cholesterol efflux will have major effects on signal transduction. Therefore, we investigated the effects of $\mathrm{HDL}$ on nuclear factor-kB (NF-kB), a major regulator of macrophage cytokine expression ${ }^{23}$. Indeed, treatment of BMDMs with rHDL resulted in significantly increased expression of NF-KB target genes (Fig. 4A). In addition, treatment of BMDMs from NF-KB luciferase reporter mice with HDL amplified the LPSinduced luciferase activity (Fig. 4B), while not affecting baseline luciferase activity, once more underpinning that the used HDL batches were endotoxinfree (Fig. 4C). More specifically, HDL as well as methyl- $\beta$-cyclodextrin induced phosphorylation (i.e. activation) of the NF-kB subunit, p65 (Fig. 4D). Using confocal imaging we determined that HDL also increased the nuclear translocation of p65 (Fig. 4E), further indicating that HDL activates NF-KB. Several upstream signaling pathways can affect NF-KB activation, including the IKK $\beta$ dependent canonical pathway, the non-canonical pathway involving NF-KB inducing kinase (NIK) and IKKa, and the largely casein kinase II (CKII) mediated atypical pathway ${ }^{21}$. Surprisingly, absence of IKKa activity (IKKa ${ }^{\mathrm{AA} / \mathrm{AA}}$ mice homozygously express an inactivatable variant of IKKa) and deficiency in IKK $\beta$ or NIK did not influence the HDL mediated changes in LPS-induced cytokine secretion of BMDMs (Fig. 4F-H), indicating that the HDL induced NF-KB activation is independent of these factors. In addition, CKII inhibition did not attenuate HDL's pro-inflammatory effect on LPS-induced cytokine secretion (Fig. 4I), excluding the involvement of the atypical NF-KB activation pathway as well. 


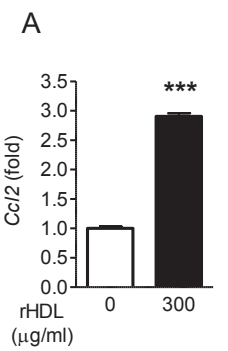

C

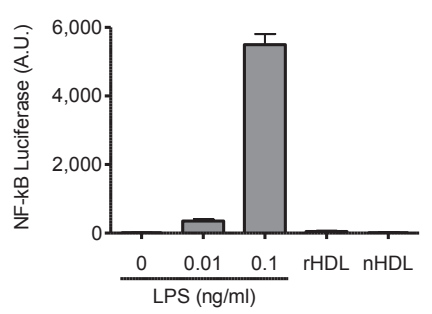

B

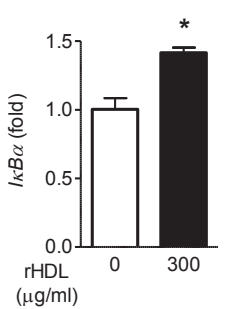

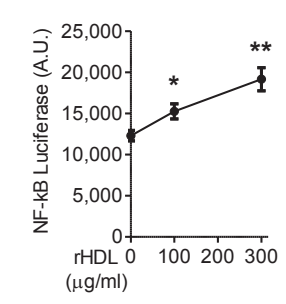

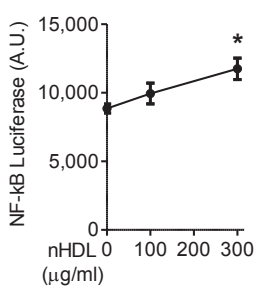

D

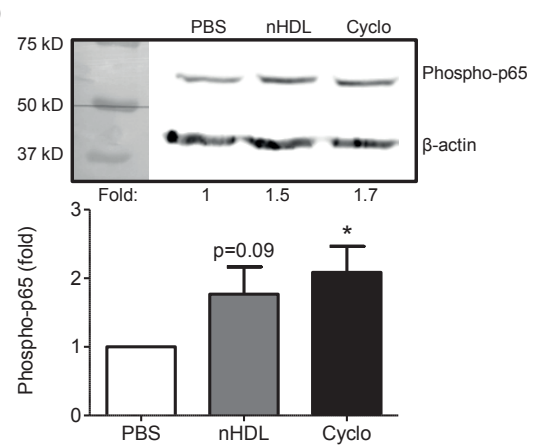

E

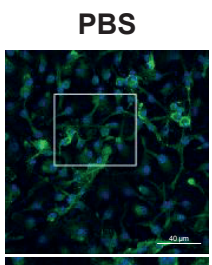

$$
\text { PBS + LPS }
$$

nHDL

nHDL + LPS
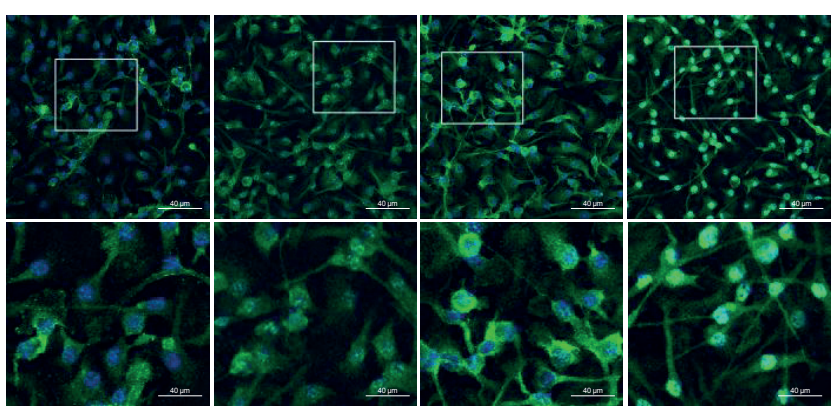

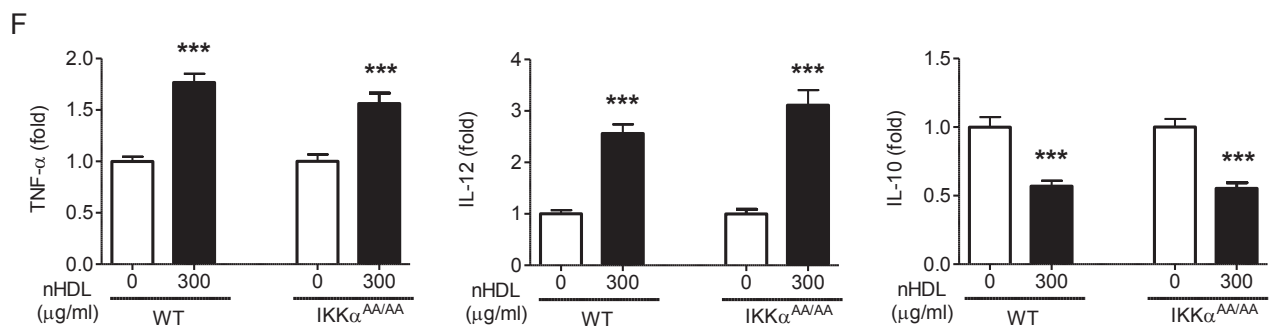

G
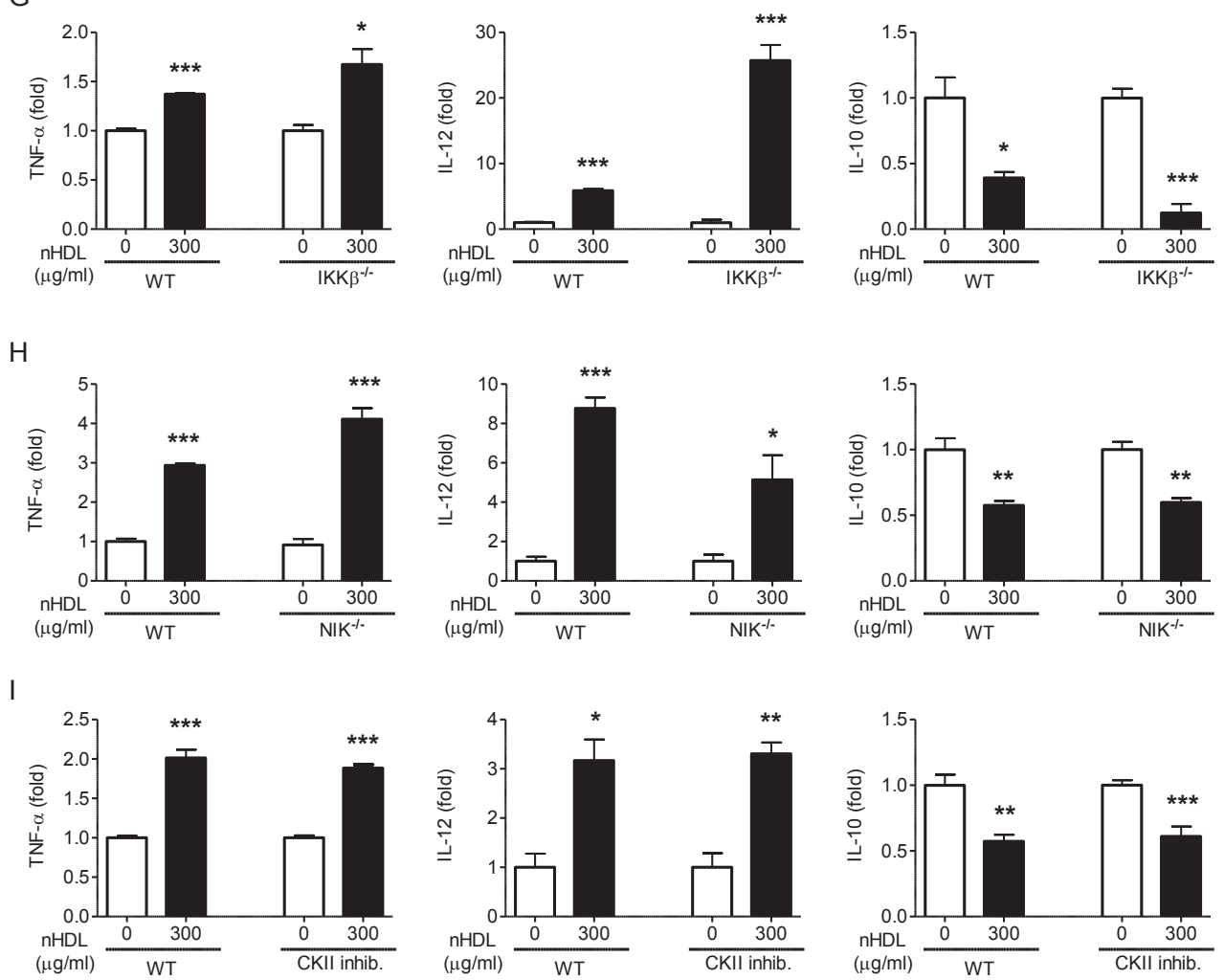
Figure 4. Macrophage pro-inflammatory HDL effects are mediated by NF-kB activation. (A) mRNA expression of NF-KB target genes in BMDMs $(n=3)$ incubated with rHDL (0-300 $\mu \mathrm{g} / \mathrm{ml}$ ) for 24 hrs. (B) Luciferase levels in BMDMs from NF-kB luciferase reporter mice $(n=3)$ pre-incubated with rHDL or nHDL $(0-300 \mu \mathrm{g} / \mathrm{ml})$ for $24 \mathrm{hrs}$, washed and stimulated with 10 $\mathrm{ng} / \mathrm{ml}$ LPS for $3 \mathrm{hrs}$. (C) Luciferase levels in BMDMs from NF-kB luciferase reporter mice $(\mathrm{n}=3)$ stimulated with $0-0.1 \mathrm{ng} / \mathrm{ml}$ LPS for $3 \mathrm{hrs}$ or incubated with rHDL or $\mathrm{nHDL}(0-300 \mu \mathrm{g} /$ $\mathrm{ml}$ ) only for $24 \mathrm{hrs}$. (D) Immunoblots showing phosphorylation of NF-kB subunit p65 (p-p65) after treatment with $300 \mu \mathrm{g} / \mathrm{ml} \mathrm{nHDL}$ for $24 \mathrm{~h}$ or $4 \%$ (vol/vol) methyl- $\beta$-cyclodextrin for $4 \mathrm{hrs}$ followed by $5 \mathrm{~min}$ stimulation with $10 \mathrm{ng} / \mathrm{ml}$ LPS. Representative immunoblot is shown with quantification of $n=5$ experiments (E) Immunofluorescent images of NF-kB subunit $\mathrm{p} 65$ after treatment with $300 \mu \mathrm{g} / \mathrm{ml} \mathrm{nHDL}$ for $24 \mathrm{hrs}$ followed by $1 \mathrm{hr}$ stimulation with $10 \mathrm{ng} / \mathrm{ml}$ LPS. Representative images of experimental $n=2$ are shown. (F-I) ELISA of cytokine production by BMDMs (all $n=3$ ) with deficiency and inhibition of major NF-KB pathway components, i.e. IKK ${ }^{A A / A A}(\mathbf{F}), I K K^{-1-}(\mathbf{G}), \mathrm{NIK}^{-1-}(\mathbf{H})$, CKII inhibited (I) BMDMs. BMDMs were pre-incubated with nHDL $(0-300 \mu \mathrm{g} / \mathrm{ml})$ for $24 \mathrm{hrs}$, washed and stimulated with LPS $(10 \mathrm{ng} / \mathrm{ml})$ for $6 \mathrm{hrs}$ (TNF- $\alpha$ and IL-10) or 24 hrs (IL-12). Casein Kinase II inhibitor $(10 \mu \mathrm{g} / \mathrm{ml})$ was added during LPS stimulation. All results are expressed as mean \pm SEM. ${ }^{*} p<0.05,{ }^{* *} p<0.01,{ }^{* * *} p<0.001$, compared to non-treated control cells. 


\section{PKC-dependent dual mechanism of HDL's pro-inflammatory effects}

A well-known lipid raft-associated kinase implicated in direct NF-KB activation 22 is protein kinase $C(P K C)$. Co-incubation of BMDMs with HDL and Ro318425, a specific PKC inhibitor, diminished the LPS-induced pro-inflammatory effects of HDL, identifying PKC as a key mediator in HDL pro-inflammatory cytokine production in BMDMs (Fig. 5A). PKC has been shown not only to induce NF-KB phosphorylation, but also Signal Transducer and Activator of Transcription (STAT) 1 phosphorylation ${ }^{23,24}$. Remarkably, deficiency of STAT1 blunted the HDL-associated augmentation of the IL-12 and IL-10 response to LPS (Fig. 5B), while it did not alter that of TNF- $\alpha$ (Fig. 5B), suggestive of differential regulation of these cytokines by HDL.

This dichotomous response led us to investigate whether HDL induced TNF- $\alpha$ production would implicate a pathway independent of the NF-KB/STAT1 axis. Our attention was drawn to the A Disintegrin And Metalloprotease (ADAM) protease family, as several members of this family, such as ADAM17 (TNF- $\alpha$ converting enzyme or TACE), and ADAM9, 10 and $19{ }^{25}$, were reported to be capable of cleaving membrane-bound pro-TNF- $\alpha$, thereby releasing active TNF- $\alpha$. Interestingly ADAMs are localized in lipid rafts and activated upon their disruption ${ }^{26,27}$. Using inhibitors of the two major ADAMs with TNF- $\alpha$ shedding activity, GI254023X (ADAM10) and GW280264X (ADAM10/ ADAM17) we confirm that indeed ADAM17 is the major enzyme responsible for TNF- $\alpha$ secretion by BMDMs since TNF- $\alpha$ production was completely blunted with combined ADAM10/ADAM17 inhibition, but not with ADAM10 inhibition alone (Fig. 5C). While HDL treatment increased TNF- $\alpha$ release in control or ADAM10-inhibited BMDMs, it was unable to enhance TNFproduction in ADAM10+ADAM17-inhibited BMDMs. The HDL-mediated increased TNF- $\alpha$ release could in turn initiate a positive feedback loop, inducing NF-KB activation ${ }^{21}$. We used the soluble TNF-Receptor (Enbrel) to capture the TNF- $\alpha$ released by BMDMs, thereby blunting this potential feedback loop. Enbrel treatment did not impact IL-12 and IL-10 responses refuting that BMDM released TNF- $\alpha$ is responsible for HDL's modulation of IL-10/IL-12 secretion (Fig. 5D). 

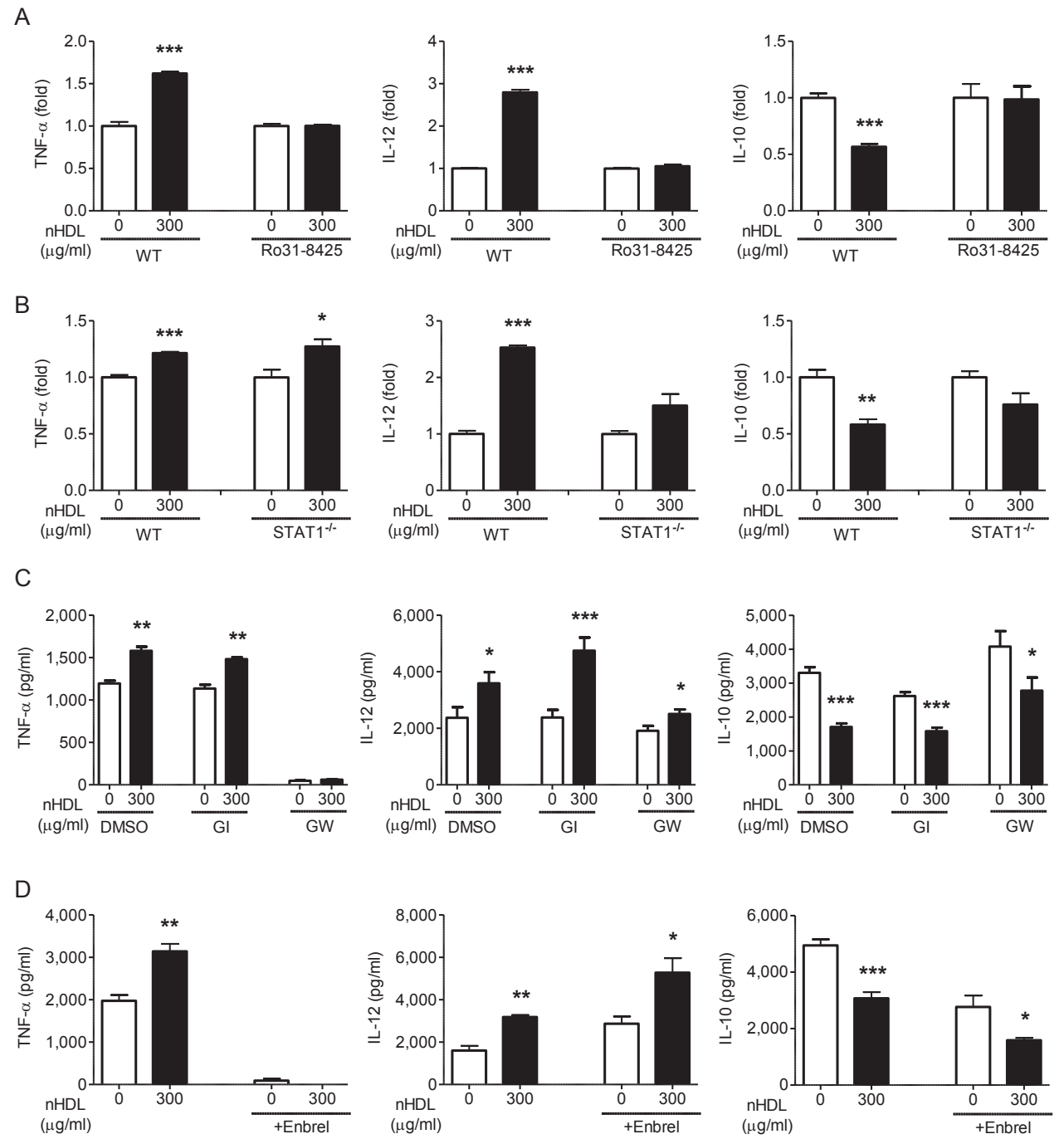

Figure 5. PKC mediates HDL pro-inflammatory effects in macrophages. (A-D) BMDMs were pre-incubated with $\mathrm{nHDL}(0-300 \mu \mathrm{g} / \mathrm{ml})$ for $24 \mathrm{hrs}$, washed and stimulated with LPS (10 $\mathrm{ng} / \mathrm{ml}$ ) for $6 \mathrm{hrs}$ (TNF- $\alpha$ and IL-10) or 24 hrs (IL-12). (A) ELISA of cytokine secretion from BMDMs treated with 0-10 $\mu \mathrm{M}$ Ro31-8425 during treatment with HDL and LPS. (B) Cytokine secretion of wildtype and STAT1-- BMDMs after treatment with HDL and LPS-stimulation as before. (C) Effect of ADAM inhibition ( $5 \mu \mathrm{M}$ GI254023X - ADAM10 or $5 \mu \mathrm{M}$ GW280264X ADAM10/17) on cytokine secretion by BMDMs. (D) ELISA of cytokine secretion from BMDMs treated as described before in the presence or absence of $10 \mu \mathrm{g} / \mathrm{ml}$ of the TNFR blocker Enbrel during all treatments. All results are expressed as mean \pm SEM. ${ }^{*} p<0.05,{ }^{* *} p<0.01$, ${ }^{* * *} p<0.001$, compared to non-treated control or wildtype cells. 


\section{Discussion}

Aside from its cholesterol efflux stimulatory capacity, cardioprotection of HDL is also attributed to its anti-inflammatory functions, which are mainly based on findings in ECs and SMCs. Here we provide in vitro and in vivo evidence for a pro-inflammatory activity of HDL in macrophages. We demonstrate that the observed pro-inflammatory effects are mediated by passive cholesterol depletion, independent of the conventional cholesterol transporters or scavenger receptors. HDL induced cholesterol depletion induces lipid raft disruption, leading to activation of the PKC-NF-KB/STAT1 axis without implicating the established NF-kB signaling pathways, thereby modulating IL-12/IL-10 cytokine production. In parallel, HDL influence ADAM protease dependent TNF- $\alpha$ release.

Immunomodulatory effects of HDL in macrophages have only been subject of a few scattered studies. In line with our observations, others have previously shown that native HDL induce TNF- $\alpha$ cytokine secretion in acetylated LDL-loaded peritoneal macrophages ${ }^{28}$. This study unfortunately did not detail the mechanism underlying HDL induced TNF- $\alpha$ secretion. A second study revealed that human lipid-free apoA-I can activate NF-kB signaling, by interacting with TLR2, TLR4 and MyD88 to induce pro-inflammatory cytokine secretion in peritoneal macrophages ${ }^{8}$. However, the physiological relevance of those findings in vivo is unclear as in blood lipid-free apoA-I will be rapidly converted into $\mathrm{HDL}{ }^{29}$, which in that study did not display any activity. Recently, a study reported overt ATF3 mediated anti-inflammatory effects of reconstituted HDL in macrophages ${ }^{9}$, which is in sharp contrast to our findings and the aforementioned studies. Their study, however, mainly focusses on reconstituted HDL, consisting of soy-bean phosphatidylcholine and ApoA-I, used at supra physiological concentrations (i.e. approx. 2-fold higher than average HDL levels in humans). Previously it has already been shown that various soy-bean components can act anti-inflammatory in macrophages ${ }^{30}$, suggesting the observed anti-inflammatory effects may be limited to HDL compounds specifically containing soy bean phospholipids/ phosphatidylcholine. In this study, we show that both 1-palmitoyl-2linoleoyl-phosphatidylcholine reconstituted ApoA-I and native HDL exert pro-inflammatory effects in macrophages, even at (sub)physiological concentrations. 
Further supporting our observations of pro-inflammatory activity of HDL in both mouse and human macrophages in vitro, peritoneal macrophages from human ApoA-I transgenic mice, with increased circulating HDL levels, had an augmented pro-inflammatory response in vivo. This seems at odds with a previous report showing reduced plasma cytokine responses in endotoxin challenged ApoA-I transgenic compared to wildtype mice ${ }^{31}$, although plasma cytokine values reflect TLR responses by a variety of cell-types and tissue sources, rather than merely macrophages.

The cardioprotective properties of HDL have been primarily ascribed to its capacity to mediate cholesterol efflux. However, ablation of ABCA1, ABCG1, SR-B1 or CD36 in macrophages did not affect the pro-inflammatory response modulation by HDL. Deficiency of SR-B1 even led to further increased TNF- $\alpha$ secretion, which is in line with previous studies in SR-B1 knockout mice ${ }^{32}$. Several studies have already alluded to the passive, cholesterol depleting and lipid raft disrupting activity of HDL in endothelial cells ${ }^{33,34}$, a notion that was confirmed in our study for BMDMs.

Lipid rafts are highly dynamic cholesterol-rich domains in the cell membrane which form platforms concentrating signaling molecules ${ }^{3}$. Indeed, NF-KB has been shown to be activated by membrane cholesterol disruption via methyl- $\beta$-cyclodextrin ${ }^{35}$. In line with this, the current study demonstrated that $\mathrm{HDL}$ induced NF-KB activation in macrophages, in contrast to our previous findings in endothelial and smooth muscle cells where HDL inhibit NF-KB signaling ${ }^{6,7}$. Surprisingly, HDL associated activation of NF-KB as we show, appears to be independent of IKKa, IKK , NIK and CKII, suggesting that none of the well-described NF-KB signaling pathways is operational in our set-up.

Our data thus point towards a direct phosphorylation and translocation of the p65 subunit of NF-kB. As previously reported, PKC $\alpha$ and $\mathrm{PKC} \zeta$ can activate NF-KB ${ }^{22}$. Importantly, PKC has also been shown to reside in lipid rafts and to be activated upon raft disruption ${ }^{36}$. In the current study we show that HDL treatment of macrophages results in PKC-dependent modulation of LPSinduced cytokine production, amplifying the inflammatory response. PKC can also directly phosphorylate STAT $1^{37}$, a major transcription factor involved in a variety of biological responses ${ }^{38}$. While HDL have not yet been linked to STAT1 activation, we clearly present conclusive evidence that HDL effects on LPS-induced cytokine secretion are partly STAT1 dependent. Intriguingly, 
we observed a regulatory dichotomy between on the one hand IL-10/IL-12 induction which appear to be STAT1 dependent and on the other hand TNF- $\alpha$ responses, which seem at least partly STAT1 independent.

Interestingly, PKC not only activates STAT1 but also specific ADAM family members ${ }^{39}$, which are instrumental in TNF- $\alpha$ secretion, such as ADAM10 and ADAM $17{ }^{25}$. Furthermore, lipid raft disruption has been shown to enhance ADAM17-dependent TNF-shedding in endothelial cells ${ }^{27,34}$. The finding that ADAM inhibition ablated TNF- $\alpha$ response to LPS in baseline as well as HDL treated macrophages, not only underpins the crucial relevance of ADAMs for TNF- $\alpha$ release, but also their involvement in the HDL augmented TNF- $\alpha$ production. Although ADAM activation associated TNF- $\alpha$ release might indirectly augment the production of the other cytokines, in our setting such positive feedback loop does not contribute significantly to the secretion of IL-12 or IL-10.

Collectively, we propose the following mode of action of HDL's immunomodulatory effects in macrophages (Fig. 6). HDL extract membrane cholesterol, thereby disrupting cholesterol rich regions called lipid rafts without the involvement of known cholesterol transporters or scavenger receptors. This in turn will activate PKC, as was reported before ${ }^{40,41}$, but will also induce ADAM protease activity, leading to increased TNF- $\alpha$ secretion ${ }^{39}$. In parallel, PKC will target STAT1 ${ }^{24}$ and NF-KB, thereby inducing II-12, while reducing IL-10 production.

In conclusion, HDL induce pro-inflammatory responses in LPS challenged macrophages in vitro and in vivo, via passive cholesterol depletion leading to activation of the PKC-NF-KB/STAT1 axis on the one hand and to ADAM protease dependent TNF- $\alpha$ release on the other hand. This pro-inflammatory response appears to be specific for macrophages, since HDL exert antiinflammatory effects in other cell-types, including ECs and VSMCs ${ }^{6,7,42,43 .}$ Macrophages are implicated in a whole range of pathologies ${ }^{44}$, including cardiovascular diseases but also autoimmune diseases, neurological disorders, metabolic complications and cancer. It is conceivable that elevated HDL levels can impact these disorders by augmenting TLR dependent proinflammatory responses. Thus, our study legitimates a thorough assessment of HDL associated effects on macrophage inflammatory responses and clinical outcome in the context of these pathologies. Considering the cell-type specificity of HDL effects, the therapeutic potential of HDL might depend on 
the type and stage of disease targeted. Regarding cardiovascular diseases, the disappointing outcome from recent clinical trials and a meta-analysis integrating data from 39 clinical trials on the efficacy of HDL targeting therapy ${ }^{45}$ underline the urgency of a better understanding of HDL's activity spectrum such as provided by our study.

Figure 6. Proposed mechanism of pro-inflammatory action of HDL in macrophages. HDL extract cholesterol from the membrane of macrophages, thereby disrupting the cholesterol rich regions called lipid rafts without involvement of known cholesterol transporters (ABCA1 and $A B C G 1)$ or scavenger receptors (SR-B1 and CD36). Cholesterol depletion activates $\mathrm{PKC}$, which stimulates two parallel pathways shown to be essential in the observed proinflammatory effects. On the one hand NF-KB is activated, leading to IL-10/IL-12 production. $\mathrm{NF}-\mathrm{KB}$ activation is independent of the traditional activation pathways and is induced either by PKC-mediated phosphorylation of p65. HDL modulation of IL-10 and IL-12 is STAT1 dependent. On the other hand HDL modulate ADAM protease activity required for TNF- $\alpha$ secretion and increased TNF- $\alpha$ release does not indirectly influence the production of IL-10/ IL-12. 


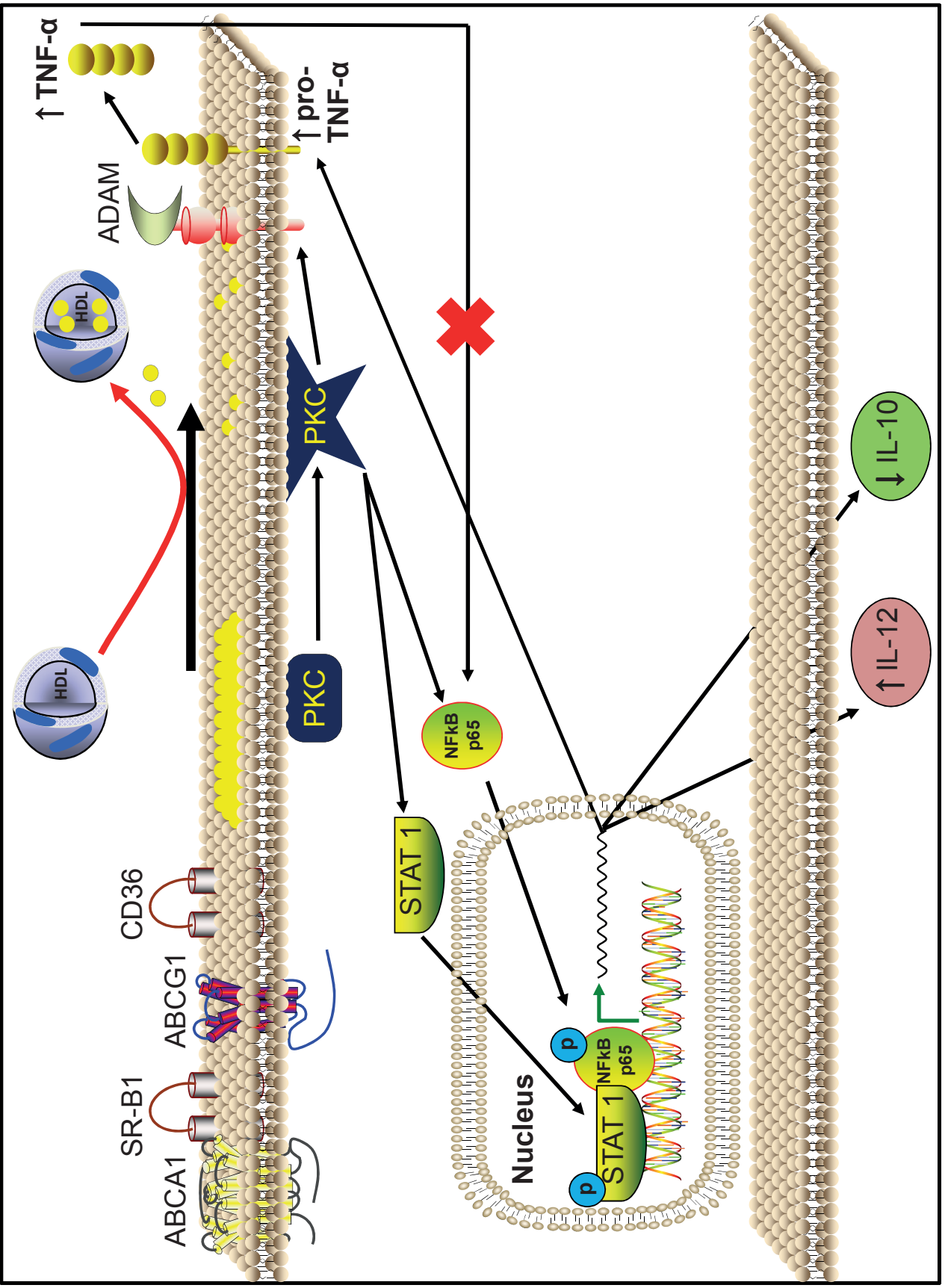




\section{Acknowledgements}

This study was supported by grants from CARIM (PhD-Award to E.P.C.V), Australian Heart Foundation (Heart Foundation Career Development Fellowship, CR07S3331 to C.A.B.), the Netherlands Organisation for Scientific Research (NWO, VENI to S.W.T and VENI916.12.039 to S.A.), ZonMw (Clinical Fellowship to S.W.T.), Dutch Arthritis Association (Research fellowship to S.A), Center for Translational Molecular Medicine (CTMM) project PREDICCt (grant 01C-104) and Dutch Heart Foundation, Dutch Diabetes Research Foundation and Dutch Kidney Foundation (PREDICCt, to D.P.Y.K.), the Netherlands Heart foundation (Dr. E. Dekker grant, 2007T034 and 2012 T079 to M.M.P.C. Donners). 


\section{References}

1. Takeuchi O, Akira S. Pattern recognition receptors and inflammation. Cell. 2010;140:805-820

2. Kawai T, Akira S. Toll-like receptors and their crosstalk with other innate receptors in infection and immunity. Immunity. 2011;34:637-650

3. Fessler MB, Parks JS. Intracellular lipid flux and membrane microdomains as organizing principles in inflammatory cell signaling. J Immunol. 2011;187:15291535

4. Barter PJ, Nicholls S, Rye KA, Anantharamaiah GM, Navab M, Fogelman AM. Antiinflammatory properties of hdl. Circ Res. 2004;95:764-772

5. Hansson GK. Inflammation, atherosclerosis, and coronary artery disease. $N$ Engl J Med. 2005;352:1685-1695

6. Bursill CA, Castro ML, Beattie DT, Nakhla S, van der Vorst E, Heather AK, et al. High-density lipoproteins suppress chemokines and chemokine receptors in vitro and in vivo. Arterioscler Thromb Vasc Biol. 2010;30:1773-1778

7. van der Vorst EP, Vanags LZ, Dunn LL, Prosser HC, Rye KA, Bursill CA. Highdensity lipoproteins suppress chemokine expression and proliferation in human vascular smooth muscle cells. FASEB J. 2012

8. Smoak KA, Aloor JJ, Madenspacher J, Merrick BA, Collins JB, Zhu X, et al. Myeloid differentiation primary response protein 88 couples reverse cholesterol transport to inflammation. Cell Metab. 2010;11:493-502

9. De Nardo D, Labzin LI, Kono H, Seki R, Schmidt SV, Beyer M, et al. High-density lipoprotein mediates anti-inflammatory reprogramming of macrophages via the transcriptional regulator atf3. Nature immunology. 2014;15:152-160

10. Weisweiler P. Isolation and quantitation of apolipoproteins a-i and a-ii from human high-density lipoproteins by fast-protein liquid chromatography. Clinica chimica acta; international journal of clinical chemistry. 1987;169:249-254

11. Matz CE, Jonas A. Micellar complexes of human apolipoprotein a-i with phosphatidylcholines and cholesterol prepared from cholate-lipid dispersions. The Journal of biological chemistry. 1982;257:4535-4540

12. Takayama M, Itoh S, Nagasaki T, Tanimizu I. A new enzymatic method for determination of serum choline-containing phospholipids. Clinica chimica acta; international journal of clinical chemistry. 1977;79:93-98

13. Wolfs IM, Stoger JL, Goossens P, Pottgens C, Gijbels MJ, Wijnands E, et al. Reprogramming macrophages to an anti-inflammatory phenotype by helminth antigens reduces murine atherosclerosis. FASEB J. 2014;28:288-299

14. Cockerill GW, Rye KA, Gamble JR, Vadas MA, Barter PJ. High-density lipoproteins 
inhibit cytokine-induced expression of endothelial cell adhesion molecules. Arterioscler Thromb Vasc Biol. 1995;15:1987-1994

15. Out R, Hoekstra M, Habets K, Meurs I, de Waard V, Hildebrand RB, et al. Combined deletion of macrophage abca1 and abcg1 leads to massive lipid accumulation in tissue macrophages and distinct atherosclerosis at relatively low plasma cholesterol levels. Arterioscler Thromb Vasc Biol. 2008;28:258-264

16. Rubin EM, Ishida BY, Clift SM, Krauss RM. Expression of human apolipoprotein a-i in transgenic mice results in reduced plasma levels of murine apolipoprotein a-i and the appearance of two new high density lipoprotein size subclasses. Proc Natl Acad Sci U S A. 1991;88:434-438

17. Brundert M, Heeren J, Merkel M, Carambia A, Herkel J, Groitl P, et al. Scavenger receptor cd36 mediates uptake of high density lipoproteins in mice and by cultured cells. J Lipid Res. 2011;52:745-758

18. Out R, Hoekstra M, Spijkers JA, Kruijt JK, van Eck M, Bos IS, et al. Scavenger receptor class $b$ type $i$ is solely responsible for the selective uptake of cholesteryl esters from hdl by the liver and the adrenals in mice. J Lipid Res. 2004;45:2088-2095

19. Soran H, Hama S, Yadav R, Durrington PN. Hdl functionality. Curr Opin Lipidol. 2012;23:353-366

20. Simons K, Toomre D. Lipid rafts and signal transduction. Nature reviews. Molecular cell biology. 2000;1:31-39

21. Perkins ND. Integrating cell-signalling pathways with nf-kappab and ikk function. Nature reviews. Molecular cell biology. 2007;8:49-62

22. Signorelli $P$, Luberto $C$, Hannun YA. Ceramide inhibition of nf-kappab activation involves reverse translocation of classical protein kinase $c(p k c)$ isoenzymes: Requirement for kinase activity and carboxyl-terminal phosphorylation of pkc for the ceramide response. FASEB J. 2001;15:2401-2414

23. Rhee SH, Jones BW, Toshchakov V, Vogel SN, Fenton MJ. Toll-like receptors 2 and 4 activate stat 1 serine phosphorylation by distinct mechanisms in macrophages. The Journal of biological chemistry. 2003;278:22506-22512

24. Salonen T, Sareila O, Jalonen U, Kankaanranta H, Tuominen R, Moilanen E. Inhibition of classical pkc isoenzymes downregulates stat1 activation and inos expression in Ips-treated murine j774 macrophages. British journal of pharmacology. 2006;147:790-799

25. Zheng Y, Saftig P, Hartmann D, Blobel C. Evaluation of the contribution of different adams to tumor necrosis factor alpha (tnfalpha) shedding and of the function of the tnfalpha ectodomain in ensuring selective stimulated shedding by the tnfalpha convertase (tace/adam17). The Journal of biological chemistry. 2004;279:42898-42906 
26. Murphy G. The adams: Signalling scissors in the tumour microenvironment. Nature reviews. Cancer. 2008;8:929-941

27. Tellier E, Canault M, Rebsomen L, Bonardo B, Juhan-Vague I, Nalbone G, et al. The shedding activity of adam 17 is sequestered in lipid rafts. Experimental cell research. 2006;312:3969-3980

28. Suzuki M, Pritchard DK, Becker L, Hoofnagle AN, Tanimura N, Bammler TK, et al. High-density lipoprotein suppresses the type $i$ interferon response, a family of potent antiviral immunoregulators, in macrophages challenged with lipopolysaccharide. Circulation. 2010;122:1919-1927

29. Rye KA, Bursill CA, Lambert G, Tabet F, Barter PJ. The metabolism and antiatherogenic properties of hdl. J Lipid Res. 2009;50 Suppl:S195-200

30. Kang JH, Sung MK, Kawada T, Yoo H, Kim YK, Kim JS, et al. Soybean saponins suppress the release of proinflammatory mediators by Ips-stimulated peritoneal macrophages. Cancer letters. 2005;230:219-227

31. Khovidhunkit W, Kim MS, Memon RA, Shigenaga JK, Moser AH, Feingold KR, et al. Effects of infection and inflammation on lipid and lipoprotein metabolism: Mechanisms and consequences to the host. J Lipid Res. 2004;45:1169-1196

32. Azzam KM, Fessler MB. Crosstalk between reverse cholesterol transport and innate immunity. Trends in endocrinology and metabolism: TEM. 2012;23:169-178

33. Norata GD, Pirillo A, Ammirati E, Catapano AL. Emerging role of high density lipoproteins as a player in the immune system. Atherosclerosis. 2012;220:11-21

34. Tellier E, Canault M, Poggi M, Bonardo B, Nicolay A, Alessi MC, et al. Hdls activate adam17-dependent shedding. Journal of cellular physiology. 2008;214:687-693

35. Flemming JA, Perkins KH, Luus L, Ferguson AR, Corley RB. Disruption of membrane cholesterol stimulates myd88-dependent nf-kappab activation in immature b cells. Cellular immunology. 2004;229:68-77

36. Steinberg SF. Structural basis of protein kinase $\mathbf{c}$ isoform function. Physiological reviews. 2008;88:1341-1378

37. Shoenfelt JL, Fenton MJ. TIr2- and tIr4-dependent activation of stat1 serine phosphorylation in murine macrophages is protein kinase c-delta-independent. Journal of endotoxin research. 2006;12:231-240

38. Ramana CV, Chatterjee-Kishore M, Nguyen H, Stark GR. Complex roles of stat1 in regulating gene expression. Oncogene. 2000;19:2619-2627

39. Killock DJ, Ivetic A. The cytoplasmic domains of tnfalpha-converting enzyme (tace/adam17) and I-selectin are regulated differently by p38 mapk and pkc to promote ectodomain shedding. The Biochemical journal. 2010;428:293-304

40. Deeg MA, Bowen RF, Oram JF, Bierman EL. High density lipoproteins stimulate mitogen-activated protein kinases in human skin fibroblasts. Arterioscler Thromb Vasc Biol. 1997;17:1667-1674 
41. Nofer JR, Kehrel B, Fobker M, Levkau B, Assmann G, von Eckardstein A. HdI and arteriosclerosis: Beyond reverse cholesterol transport. Atherosclerosis. 2002;161:1-16

42. Cheng AM, Handa P, Tateya S, Schwartz J, Tang C, Mitra P, et al. Apolipoprotein a-i attenuates palmitate-mediated nf-kappab activation by reducing toll-like receptor-4 recruitment into lipid rafts. PLoS One. 2012;7:e33917

43. McGrath KC, Li XH, Puranik R, Liong EC, Tan JT, Dy VM, et al. Role of 3betahydroxysteroid-delta 24 reductase in mediating antiinflammatory effects of high-density lipoproteins in endothelial cells. Arterioscler Thromb Vasc Biol. 2009;29:877-882

44. Murray PJ, Wynn TA. Protective and pathogenic functions of macrophage subsets. Nat Rev Immunol. 2011;11:723-737

45. Rader DJ, Tall AR. The not-so-simple hdl story: Is it time to revise the hdl cholesterol hypothesis? Nature medicine. 2012;18:1344-1346 


\section{Supplemental Figure}

A

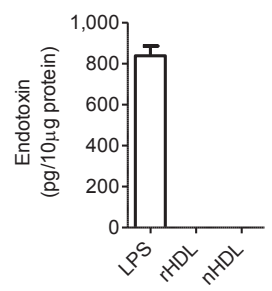

B
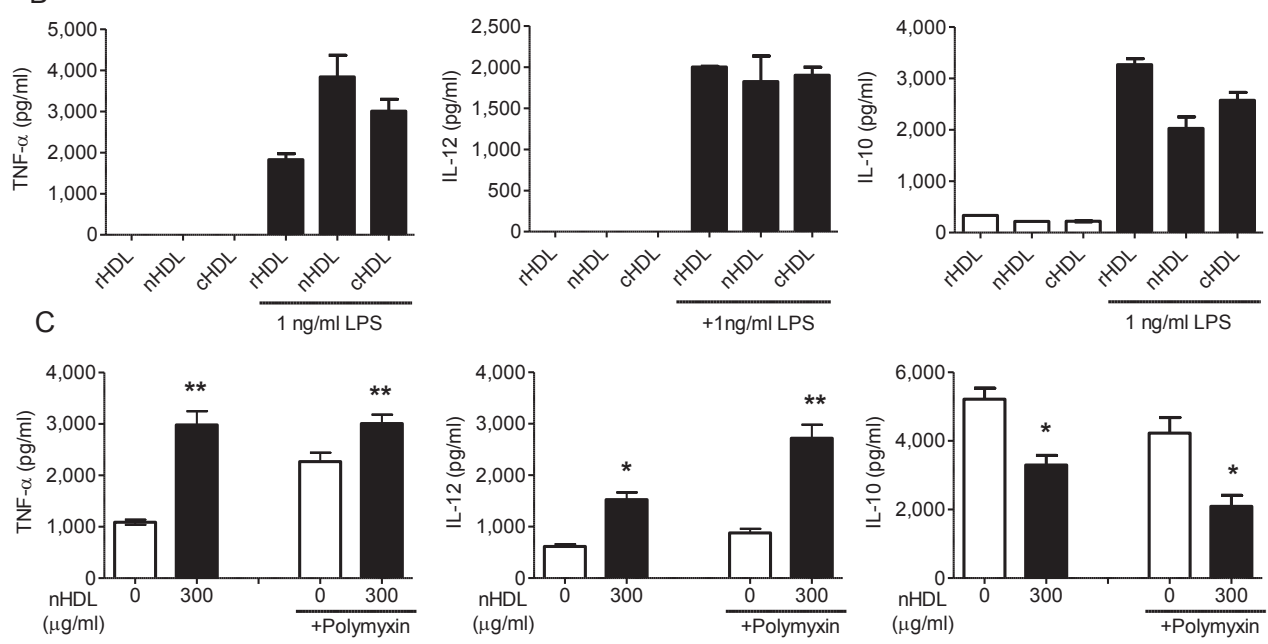

Supplemental Figure 1. HDL do not show any signs of contamination. (A) Endotoxin concentration per $10 \mu \mathrm{g}$ protein of BMDMs $(\mathrm{n}=3)$ incubated for $24 \mathrm{hrs}$ with $10 \mathrm{ng} / \mathrm{ml}$ LPS, rHDL or $\mathrm{nHDL}$ (both $300 \mu \mathrm{g} / \mathrm{ml}$ ) was measured using Limulus testing. (B) TNF- $\alpha, \mathrm{IL}-12$ and IL-10 production by unstimulated or LPS $(1 \mathrm{ng} / \mathrm{ml})$ stimulated BMDMs $(\mathrm{n}=2)$ pre-incubated with $300 \mu \mathrm{g} / \mathrm{ml} \mathrm{nHDL}$ for $24 \mathrm{hrs}$. (C) ELISA of BMDMs (n=3) pre-incubated for $24 \mathrm{hrs}$ with $\mathrm{nHDL}$ in the absence or presence of polymyxin $\mathrm{B}$, washed and stimulated with $10 \mathrm{ng} / \mathrm{ml} \mathrm{LPS}$. All results are expressed as mean \pm SEM. ${ }^{*} p<0.05$, ${ }^{* *} p<0.01$, compared to non-HDL preincubated control cells. 


\section{Chapter 8}

\section{General Discussion}




\section{Introduction}

Vascular inflammation is crucial in the development of various pathologies including atherosclerosis, which is the underlying cause of cardiovascular diseases like myocardial infarction and stroke. After years of research, to date the available drugs or therapies to prevent, cure or reverse atherosclerosis in all patients are still limited. In this thesis we focussed on two hallmark features of atherosclerosis: inflammation and lipid metabolism. More specifically, in the first part of this thesis we focussed on ADAM dependent vascular inflammation, whereas in the second part we investigated the HDL dependent modulation of vascular inflammation.

The main findings of this thesis are:

- Although ADAM8 expression is associated with atherosclerotic lesion progression, its deficiency in leukocytes did not influence plaque development (Chapter 3).

- Myeloid ADAM10 deficiency improves atherosclerotic plaque stability by influencing inflammation and fibrosis (Chapter 4), while its deficiency in endothelial cells aggravates atherosclerotic lesion development and progression (Chapter 5).

- High density lipoproteins suppress chemokine expression and proliferation of vascular smooth muscle cells, in a SR-B1 and NF-KB dependent manner (Chapter 6).

- Conversely, in macrophages high density lipoproteins exert pro-inflammatory effects, by passive cholesterol depletion and by PKC-dependent activation of NF-KB / STAT1 and ADAM pathways (Chapter 7). 


\section{A Disintegrin And Metalloproteinases}

\section{Cell specific effects of ADAM10 on atherosclerosis}

Chapters 4 and 5 clearly indicate a cell-type specific role of ADAM10 in atherosclerosis development. We focussed on ADAM10, since this protease has many substrates relevant for atherosclerosis (Chapter 2) and our group already showed that ADAM10 is associated with plaque progression in humans ${ }^{1}$. Where myeloid ADAM10 deletion only results in a mild modulation of plaque stability (Chapter 4), endothelial ADAM10 deficiency considerably increases the lesion size and progression (Chapter 5). This suggests that ADAM10 in endothelium has strong atheroprotective effects, which most likely will overrule the less beneficial effects in myeloid cells.

The endothelium is a crucial barrier that needs to be crossed by inflammatory cells to contribute to atherosclerosis. In this process, various adhesion molecules, chemokines but also junction molecules play a crucial role ${ }^{2}$ and it has been shown that ADAMs play a role in the cleavage of various involved proteins ${ }^{1,3-6}$. Since endothelial ADAM10 already plays an important role in the initial phase of disease development, it is expected to primarily influence disease initiation.

Myeloid ADAM10 deficiency, however, mainly influences the disease development in a somewhat later stage. Macrophages, in the lesions mainly foam cells, secrete various cytokines, but also matrix degrading enzymes 7. This way it contributes to leukocyte recruitment, but also to plaque stabilization by influencing the fibrous cap. Especially this influence on plaque fibrosis explains the observed differences in the myeloid ADAM10 deficient mice. Depending on the disease stage and the targeted cell-type involved, conditional ADAM10 deficiency may thus have different effects on atherosclerosis.

\section{ADAM8 in atherosclerosis}

A relatively unexplored ADAM family member is ADAM8. Interestingly a recent cohort study showed that a polymorphism of ADAM8 significantly increases the risk of myocardial infarction ${ }^{8}$. Surprisingly, we did not find any causal role for hematopoietic ADAM8 on lesion development (Chapter 3). To date, there are still only few substrates described for ADAM8, for example $\mathrm{CX}_{3} \mathrm{CL} 1$ and L-selectin ${ }^{9}$. For better understanding of the function of this protease 
it is crucial that more substrates are identified. A proteomics analysis of its sheddome, like we performed in chapter 5 , could be very helpful in this regard. The observed results in chapter 3 do not necessary rule out an involvement of ADAM8 in the pathophysiology of atherosclerosis, especially since ADAM8 exist in two active forms, namely membrane bound and a soluble form ${ }^{10}$. This makes the investigation of the true role of ADAM8 in atherosclerosis, and especially the involved mechanisms more challenging. The existence of a soluble ADAM8, combined with many unknown substrates, makes it very plausible that ADAM8 on, or secreted by other cell-types, like endothelial cells and smooth muscle cells have compensated for the loss of ADAM8 expression on ADAM8 deficient hematopoietic cells. This interaction could either be via membrane bound ADAM8 on two different cell-types, directly influencing each other, or by secretion of soluble ADAM8.

To exclude this possible compensation, total body ADAM8 deficient mice, which we have rendered atherosclerosis prone by AAV aided PCSK9 gene transfer ${ }^{11}$, can be used to determine the role of ADAM8 in atherosclerosis. This gene transfer approach will circumvent time-consuming breedings with atherosclerosis-prone $\mathrm{LDLr}^{-/}$or $\mathrm{ApoE}^{-/-}$mice. In addition, a bone marrow transplantation of ADAM8 wildtype or deficient hematopoetic cells into either wildtype or total body ADAM8 deficient mice, injected with PCSK9-AAV, may add to unveil the specific role of cell-specific ADAM8 and soluble ADAM8 in this pathogenesis.

Several studies have already been performed with total body ADAM8 deficient mice in other diseases, like cancer, where ADAM8 targeting resulted in reduced tumor load and metastasis ${ }^{12}$. Furthermore, ADAM8 has also been implicated in the development of inflammatory diseases. ADAM8 plays for example a pro-inflammatory role in an ovalbumin-induced asthma model or favours allergen-induced airway inflammation ${ }^{13,14}$. Both studies used total body ADAM8 deficient mice, thereby excluding possible confounding effects of soluble ADAM8, further supporting the hypothesis that ADAM8 plays a role in atherosclerosis but that full knockout models have to be used to elucidate this. 


\section{The ADAMs family: redundancy or complementarity?}

Other ADAMs have also been implicated in the shedding of relevant molecules for atherosclerosis (Chapter 2). One of the most important and most closely related to ADAM10, is ADAM17. It has been shown that these two ADAMs share many substrates and can compensate for each other's function to a certain extent, although this was mainly demonstrated in vitro ${ }^{15}$. In addition, total body knockout mice of ADAM10 and ADAM17 are embryonically lethal, due to defects in vasculogenesis or cardiac development respectively ${ }^{16}$, ${ }^{17}$, suggesting that full redundancy is not present in vivo, though partial redundancy may still occur. Also for ADAM17 many substrates relevant for atherosclerosis have been described such as TNF- $\alpha$, VCAM-1 and ICAM1 , suggesting important roles for ADAM17 in atherosclerosis development. This is supported by the fact that ADAM17 expression has been associated with atherosclerosis resistance in mice ${ }^{18}$. It would be very interesting to also investigate the effect of cell-specific ADAM17 deficiency in atherosclerosis. Recently is has already been shown that macrophage ADAM17 plays a crucial role in efferocytosis regulation in vivo ${ }^{19}$. Additionally, endothelial and smooth muscle cell ADAM17 have been shown to promote acute inflammation ${ }^{20,}{ }^{21}$. Based on these studies in combination with the large amount of publications showing ADAM17 substrates, also listed in chapter 2, it would be expected that ADAM17 deficiency would dramatically influence atherosclerosis. A recent publication by Pruessmeyer et al. suggests that leukocyte-expressed ADAM10, but not ADAM17 is essential for leukocyte recruitment at least in a model of acute pulmonary inflammation ${ }^{22}$. Whether there are also cellspecific differences on lesion outcome with ADAM17 deficiency remains to be determined.

\section{Domain dependent functions/metalloprotease activity vs integrin- related functions}

To date, 12 proteolytically active ADAMs have been described ${ }^{23}$, including ADAM8, -10 and -17 . Besides these three ADAMs, also other active ADAMs have at least been associated with atherosclerosis. ADAM9 and ADAM33 for example are expressed in human atherosclerotic lesions ${ }^{24,25}$, and polymorphisms of the latter have been associated with atherosclerosis development ${ }^{24}$. For ADAM15, a causal relationship is even described, showing that overexpression of ADAM15 reduced atherosclerosis in rabbits ${ }^{26}$. Next to the 12 proteolytically active ADAMs, over 28 ADAMs have been described, which are proteolytically inactive. Besides the metalloproteinase domain, the ADAMs also contain a disintegrin domain, capable of binding 
to various integrins ${ }^{27}$. Integrins are involved in atherosclerosis related processes, like leukocyte adhesion and transmigration ${ }^{28}$. Though, the precise functional consequences of this ADAMs-integrins interaction remains an exciting area of future research. To date, some interactions have already been described. ADAM8 for example has been shown to stimulate osteoclast formation by its interaction with $\alpha 9 \beta 1$-integrin ${ }^{29}$. Additionally, ADAM9 binds to various integrins ${ }^{30}$, such as $\beta 1$ integrins, an interaction which increases keratinocyte migration leading to accelerated wound repair ${ }^{31,32}$. Recently, it has also been shown that ADAM10 promotes integrin activation, in particular a5-integrin, although it is not clear whether this involves direct binding via the disintegrin domain ${ }^{22}$. Finally, the disintegrin domain of ADAM15 inhibits airway smooth muscle cell adhesion and migration ${ }^{33}$. The relevance of these interactions for atherosclerosis still have to be determined, as well as the evaluation of proteolytically inactive ADAMs. This could open a whole new field of investigation, including many more proteases that could influence leukocyte adhesion and migration and thereby atherosclerosis development by their disintegrin domain.

\section{Regulation of ADAM functions}

ADAM activation is regulated at various levels, e.g. at transcriptional and post-transcriptional level, but also by membrane localization ${ }^{34}$.

Transciptional and post-transcriptional regulation.

Especially in various pathological conditions increased expression levels of various ADAMs have been described ${ }^{35}$. However, ADAM proteases are produced as latent enzymes and are only activated after removal of the prodomain ${ }^{23}$. Another post-transcriptional regulation method is by tissue inhibitors of metalloproteases (TIMPs), where for example TIMP1 blocks ADAM10 activation and TIMP3 inhibits both ADAM10 and ADAM17 ${ }^{36}$. Recently, it became clear that integrins can not only act as binding partners for ADAMs, but are also able to regulate ADAMs activity. More specifically, $\alpha 5 \beta 1$ integrin has been shown to inhibit and thereby regulate ADAM17 activity in kidney mesangial cells ${ }^{37}$. It would be very interesting to investigate whether this regulation is ADAM or cell-type specific or is more generally present, to give more insight into the regulation of ADAMs. 
Regulation by membrane localization

Another level of regulation involves the membrane localization of ADAMs, which can be regulated by lipid rafts. These are, cholesterol rich areas in the membrane where many receptors and signaling molecules cluster together ${ }^{38}$. Tellier et al. showed that also ADAM17 is preferentially present in these rafts ${ }^{38}$. Furthermore, they observed that disruption of these rafts led to increased shedding of TNF- $\alpha$, a main substrate of ADAM17. Disruption of the lipid rafts is then necessary to bring the ADAM and its substrate in each other's vicinity. Various lipid raft disrupting agents have already been described, like unsaturated free-fatty acids that may be liberated from LDL ${ }^{39}$. In this thesis, we showed that HDL can also disrupt these lipid rafts. As expected, since ADAM17 is the main protease responsible for TNF- $\alpha$ shedding, we observed reduced TNF- $\alpha$ release upon ADAM17 blockage, however it remains to be investigated whether HDL directly influences ADAM17 activity or that this activation is also mediated by lipid raft disruption and thereby relocalization of ADAM17. Recently, another membrane domain became of interest for ADAMs regulation, i.e. the tetraspanin-enriched microdomains. Tetraspanin CD9 was shown to be associated with ADAM17 on the surface of endothelial cells and leukocytes, where CD9 overexpression decreased ADAM17 activity and mediated shedding of TNF- $\alpha$ and ICAM- ${ }^{40}$. Also other interactions have already been described, like the regulation of ADAM10-mediated amyloid precursor protein shedding by tetraspanin $12{ }^{41}$ and TNF- $\alpha$ and ADAM10dependent cleavage of epidermal growth factor by tetraspanins CD9, CD81 and CD82 ${ }^{42}$. However, the precise in vivo relevance of the tetraspaninADAMs interaction has to be further investigated. 


\section{High Density Lipoproteins}

\section{Cell-specific and disease-specific effects of HDL}

Our studies are the first to demonstrate clear cell-type specific effects of HDL on inflammation. The most striking difference is that concordant with the current notion we observed significant anti-inflammatory effects of HDL in SMCs, (Chapter 6), while in macrophages we surprisingly observed proinflammatory HDL effects (Chapter 7). The SMC data align well with previous studies which showed that HDL has anti-inflammatory effects on endothelial cells and monocytes amongst others by inhibiting chemokine secretion ${ }^{43}$. This discrepancy makes cell-type specific HDL research warranted.

\section{Pro-inflammatory effects of HDL in macrophages}

Looking at the systemic effects of HDL, it is well described that HDL protects against cardiovascular diseases such as atherosclerosis 44,45 . This is illustrated by the observation that infusion of HDL into cholesterol-fed rabbits resulted in regression of atherosclerotic lesions ${ }^{46}$. Moreover, Rubin et al. showed that overexpression of human ApoA-I, the main constituent of HDL, resulted in high plasma $H D L$ levels in mice and inhibited early atherogenesis 47. However, these and other studies in this field of research do not specifically focus on particular cell-types, like the inflammatory status of macrophages in these lesions for example. Although for atherosclerosis the balance between the contradicting cell-type specific HDL effects seems to shift toward the anti-inflammatory and protective side, this does not necessarily hold true for other pathologies. Rheumatoid arthritis (RA) for example is mainly driven by TNF- $\alpha$, which is predominantly produced by macrophages ${ }^{48}$. Since we show that HDL significantly increases TNF- $\alpha$ secretion in macrophages, it would be crucial to further investigate the impact of $\mathrm{HDL}$ on a primarily macrophage driven pathology like RA. Also for infections, especially the HDL effects on macrophages are very interesting, since their response to invading pathogens is regulated by toll-like receptors (TLRs) ${ }^{49}$. This TLR response is amplified by HDL conditioning (Chapter 7 ). It has been shown for many infections, like the macrophage activating gram-negative bacteria P. aeruginosa, that TLR signaling is crucial for the defence against these pathogens ${ }^{50,51}$. Augmenting the TLR response to pathogenic stimuli in this setting may well improve bacterial clearance and thus inhibit disease development. This could not only be the case for infectious diseases, but also for example for asthma where TLR signaling plays a crucial role ${ }^{52}$. 


\section{Anti-inflammatory HDL effects in SMCs}

Such disease specific effects can also be proposed for HDL effects on SMCs, in particular on SMC proliferation. We showed that HDL reduces SMC proliferation (Chapter 6), which can be beneficial for neointimal hyperplasia, which occurs often after stent placement in CVD patients ${ }^{53}$. The thickening is mainly caused by hyperproliferation of SMCs ${ }^{54}$. Reduction of this proliferation may thus prevent stent occlusion and increase the patency. Clinical studies showed that patients with higher HDL levels indeed have improved stent patency at one year following stent placement ${ }^{55}$, conceivably at least partly due to this inhibited SMC proliferation. However, in the context of atherosclerosis, inhibited SMC proliferation in more advanced lesions will result in a thinner fibrous cap and thus a more instable lesion that is more prone to rupture and cause thrombus formation. This supports the notion that HDL effects should not merely be investigated systemically, but more efforts should be made to determine the combined cell-type as well as disease-type specific effects of HDL.

\section{Focus on HDL composition}

Next to dissecting cell-specific responses to HDL, it is also very important to further investigate HDL composition as determinant of its inflammatory effects. Already in many studies various types and compositions of HDL have been used, like lipid free ApoA-I, reconstituted HDL, native HDL or ApoA-I mimetic peptides. Further research elucidating the specific effects of all of these subtypes is very important, especially since comparison of our study of HDL effects in macrophages with a recently published study by De Nardo et al. ${ }^{56}$ possibly indicates that HDL composition, in particular phospholipid composition can influence the outcome. Their study namely showed that HDL, composed of soybean derived phospholipids, mediates anti-inflammatory effects in macrophages, directly contradicting our results. It has already been shown that soy-bean components have anti-inflammatory effects on macrophages ${ }^{57}$, further supporting the fact that phospholipid composition might be a critical factor involved in the HDL effects. To date, still a lot of the observed HDL effects cannot exactly be related to specific HDL constituents ${ }^{58}$, which should be a major focus of future HDL research. 


\section{Therapeutic implications}

Already since the Framingham Heart Study in 1977 that showed an important inverse relationship between $\mathrm{HDL}$ and the risk of a cardiovascular event ${ }^{59}$, there has been great interest in HDL as therapeutic approach to prevent cardiovascular complications. To date, already various clinical trials have been conducted using different approaches to target the HDL metabolism ${ }^{60}$, ${ }^{61}$. However, many of these clinical trials did not show the expected beneficial effects of HDL therapy. Based on findings in this thesis, showing contradictory effects of HDL on smooth muscle cell versus macrophage activation it is plausible that systemically these effects, at least partially, counterbalance each other, thereby limiting the effectiveness of the HDL treatment. These cell-type specific effects of $\mathrm{HDL}$ can also have major implications for treatment effectiveness in specific pathologies, since in pathologies like RA or bacterial infections macrophages play a crucial role whereas in neointimal hyperplasia SMCs are more important. Next to these cell-type and thus disease-specific effects of HDL, it is crucial that future research focusses on the elucidation of the relationship between observed HDL effects and HDL composition, enabling pharmaceutical companies to develop more targeted HDL-based approaches. 


\section{Overall future perspectives}

For both ADAM10 as well as HDL this thesis demonstrates cell-specific effects on various processes involved in vascular inflammation. This is of particular interest for the development of targeted therapeutic approaches, since by specifically targeting certain cell-types one could modulate the outcome in a disease-related and personalized manner. Further research, however is needed to fully elucidate these effects in vivo and in other diseases. Regarding ADAMs targeting, research must first gain more insight in the precise regulation of ADAMs and their specificity and redundancy in vivo. To date, still much is unclear and unexplored. This will be crucial for more specific ADAMs-based therapies, since for some ADAMs compensatory mechanisms by other ADAMs have already been described, like ADAM10 and ADAM17 in certain conditions. For HDL-based therapy it would be very important to further investigate the effects of specific HDL types and HDL constituents. As described, this can have a great influence on the specific outcome and can therefore influence therapeutic efficacy. Additionally, for ADAMs as well as HDL efforts should be made to translate the observed results to humans. Especially for HDL there could be major difference in outcome between mice and humans, since the lipid metabolism of the mouse is very different as they lack CETP ${ }^{62}$. Additional experiments in other animal models, like rabbits or even human materials can overcome this limitation. Taken together, this thesis showed various cell-type specific effects of ADAMs and HDL on vascular inflammation, although research in this field remains highly interesting to fully elucidate all remaining aspects. 


\section{References}

1. Donners MM, Wolfs IM, Olieslagers S, Mohammadi-Motahhari Z, Tchaikovski V, Heeneman $S$, et al. A disintegrin and metalloprotease 10 is a novel mediator of vascular endothelial growth factor-induced endothelial cell function in angiogenesis and is associated with atherosclerosis. Arteriosclerosis, thrombosis, and vascular biology. 2010;30:2188-2195

2. Hansson GK, Hermansson A. The immune system in atherosclerosis. Nature immunology. 2011;12:204-212

3. Garton KJ, Gough PJ, Philalay J, Wille PT, Blobel CP, Whitehead RH, et al. Stimulated shedding of vascular cell adhesion molecule 1 (vcam-1) is mediated by tumor necrosis factor-alpha-converting enzyme (adam 17). The Journal of biological chemistry. 2003;278:37459-37464

4. Koenen RR, Pruessmeyer J, Soehnlein O, Fraemohs L, Zernecke A, Schwarz N, et al. Regulated release and functional modulation of junctional adhesion molecule a by disintegrin metalloproteinases. Blood. 2009;113:4799-4809

5. Ludwig A, Weber C. Transmembrane chemokines: Versatile 'special agents' in vascular inflammation. Thrombosis and haemostasis. 2007;97:694-703

6. Tsakadze NL, Sithu SD, Sen U, English WR, Murphy G, D'Souza SE. Tumor necrosis factor-alpha-converting enzyme (tace/adam-17) mediates the ectodomain cleavage of intercellular adhesion molecule-1 (icam-1). The Journal of biological chemistry. 2006;281:3157-3164

7. Moore KJ, Sheedy FJ, Fisher EA. Macrophages in atherosclerosis: A dynamic balance. Nature reviews. Immunology. 2013;13:709-721

8. Raitoharju E, Seppala I, Levula M, Kuukasjarvi P, Laurikka J, Nikus K, et al. Common variation in the adam 8 gene affects serum sadam 8 concentrations and the risk of myocardial infarction in two independent cohorts. Atherosclerosis. 2011;218:127-133

9. Dreymueller D, Uhlig S, Ludwig A. Adam-family metalloproteinases in lung inflammation - potential therapeutic targets. American journal of physiology. Lung cellular and molecular physiology. 2014:ajplung 0029402014

10. Higuchi Y, Yasui A, Matsuura K, Yamamoto S. Cd156 transgenic mice. Different responses between inflammatory types. Pathobiology : journal of immunopathology, molecular and cellular biology. 2002;70:47-54

11. Bjorklund MM, Hollensen AK, Hagensen MK, Dagnaes-Hansen F, Christoffersen C, Mikkelsen JG, et al. Induction of atherosclerosis in mice and hamsters without germline genetic engineering. Circulation research. 2014;114:1684-1689

12. Romagnoli M, Mineva ND, Polmear M, Conrad C, Srinivasan S, Loussouarn D, et al. 
Adam8 expression in invasive breast cancer promotes tumor dissemination and metastasis. EMBO molecular medicine. 2014;6:278-294

13. Naus S, Blanchet MR, Gossens K, Zaph C, Bartsch JW, McNagny KM, et al. The metalloprotease-disintegrin adam8 is essential for the development of experimental asthma. American journal of respiratory and critical care medicine. 2010;181:1318-1328

14. Paulissen G, Rocks N, Gueders MM, Bedoret D, Crahay C, Quesada-Calvo F, et al. Adam-8, a metalloproteinase, drives acute allergen-induced airway inflammation. European journal of immunology. 2011;41:380-391

15. Le Gall SM, Bobe P, Reiss K, Horiuchi K, Niu XD, Lundell D, et al. Adams 10 and 17 represent differentially regulated components of a general shedding machinery for membrane proteins such as transforming growth factor alpha, I-selectin, and tumor necrosis factor alpha. Molecular biology of the cell. 2009;20:1785-1794

16. Jorissen E, Prox J, Bernreuther C, Weber S, Schwanbeck R, Serneels L, et al. The disintegrin/metalloproteinase adam10 is essential for the establishment of the brain cortex. The Journal of neuroscience : the official journal of the Society for Neuroscience. 2010;30:4833-4844

17. Shi W, Chen H, Sun J, Buckley S, Zhao J, Anderson KD, et al. Tace is required for fetal murine cardiac development and modeling. Developmental biology. 2003;261:371-380

18. Holdt LM, Thiery J, Breslow JL, Teupser D. Increased adam17 mrna expression and activity is associated with atherosclerosis resistance in Idl-receptor deficient mice. Arteriosclerosis, thrombosis, and vascular biology. 2008;28:10971103

19. Driscoll WS, Vaisar T, Tang J, Wilson CL, Raines EW. Macrophage adam17 deficiency augments cd36-dependent apoptotic cell uptake and the linked anti-inflammatory phenotype. Circulation research. 2013;113:52-61

20. Dreymueller D, Martin C, Kogel T, Pruessmeyer J, Hess FM, Horiuchi K, et al. Lung endothelial adam17 regulates the acute inflammatory response to lipopolysaccharide. EMBO molecular medicine. 2012;4:412-423

21. Dreymueller D, Martin C, Schumacher J, Groth E, Boehm JK, Reiss LK, et al. Smooth muscle cells relay acute pulmonary inflammation via distinct adam17/ erbb axes. Journal of immunology. 2014;192:722-731

22. Pruessmeyer J, Hess FM, Alert H, Groth E, Pasqualon T, Schwarz N, et al. Leukocytes require adam 10 but not adam 17 for their migration and inflammatory recruitment into the alveolar space. Blood. 2014;123:4077-4088

23. Reiss K, Saftig P. The "a disintegrin and metalloprotease" (adam) family of sheddases: Physiological and cellular functions. Seminars in cell \& developmental biology. 2009;20:126-137 
24. Holloway JW, Laxton RC, Rose-Zerilli MJ, Holloway JA, Andrews AL, Riaz Z, et al. Adam33 expression in atherosclerotic lesions and relationship of adam33 gene variation with atherosclerosis. Atherosclerosis. 2010;211:224-230

25. Oksala N, Levula M, Airla N, Pelto-Huikko M, Ortiz RM, Jarvinen O, et al. Adam-9, adam-15, and adam-17 are upregulated in macrophages in advanced human atherosclerotic plaques in aorta and carotid and femoral arteries--tampere vascular study. Annals of medicine. 2009;41:279-290

26. Bultmann A, Li Z, Wagner S, Gawaz M, Ungerer M, Langer H, et al. Loss of protease activity of adam15 abolishes protective effects on plaque progression in atherosclerosis. International journal of cardiology. 2011;152:382-385

27. Bridges LC, Bowditch RD. Adam-integrin interactions: Potential integrin regulated ectodomain shedding activity. Current pharmaceutical design. 2005; $11: 837-847$

28. Galkina E, Ley K. Vascular adhesion molecules in atherosclerosis. Arteriosclerosis, thrombosis, and vascular biology. 2007;27:2292-2301

29. Rao H, Lu G, Kajiya H, Garcia-Palacios V, Kurihara N, Anderson J, et al. Alpha9beta1: A novel osteoclast integrin that regulates osteoclast formation and function. Journal of bone and mineral research : the official journal of the American Society for Bone and Mineral Research. 2006;21:1657-1665

30. Mahimkar RM, Visaya O, Pollock AS, Lovett DH. The disintegrin domain of adam9: A ligand for multiple beta1 renal integrins. The Biochemical journal. 2005;385:461-468

31. Mauch C, Zamek J, Abety AN, Grimberg G, Fox JW, Zigrino P. Accelerated wound repair in adam-9 knockout animals. The Journal of investigative dermatology. 2010;130:2120-2130

32. Zigrino P, Steiger J, Fox JW, Loffek S, Schild A, Nischt R, et al. Role of adam9 disintegrin-cysteine-rich domains in human keratinocyte migration. The Journal of biological chemistry. 2007;282:30785-30793

33. Lu D, Xie S, Sukkar MB, Lu X, Scully MF, Chung KF. Inhibition of airway smooth muscle adhesion and migration by the disintegrin domain of adam-15. American journal of respiratory cell and molecular biology. 2007;37:494-500

34. Duffy MJ, McKiernan E, O'Donovan N, McGowan PM. The role of adams in disease pathophysiology. Clinica chimica acta; international journal of clinical chemistry. 2009;403:31-36

35. Murphy G. Regulation of the proteolytic disintegrin metalloproteinases, the 'sheddases'. Seminars in cell \& developmental biology. 2009;20:138-145

36. Reiss K, LudwigA, Saftig P. Breaking up the tie: Disintegrin-like metalloproteinases as regulators of cell migration in inflammation and invasion. Pharmacology \& therapeutics. 2006;111:985-1006 
37. Gooz P, Dang Y, Higashiyama S, Twal WO, Haycraft CJ, Gooz M. A disintegrin and metalloenzyme (adam) 17 activation is regulated by alpha5beta1 integrin in kidney mesangial cells. PloS one. 2012;7:e33350

38. Tellier E, Canault M, Rebsomen L, Bonardo B, Juhan-Vague I, Nalbone G, et al. The shedding activity of adam 17 is sequestered in lipid rafts. Experimental cell research. 2006;312:3969-3980

39. Reiss K, Cornelsen I, Husmann M, Gimpl G, Bhakdi S. Unsaturated fatty acids drive disintegrin and metalloproteinase (adam)-dependent cell adhesion, proliferation, and migration by modulating membrane fluidity. The Journal of biological chemistry. 2011;286:26931-26942

40. Gutierrez-Lopez MD, Gilsanz A, Yanez-Mo M, Ovalle S, Lafuente EM, Dominguez C, et al. The sheddase activity of adam17/tace is regulated by the tetraspanin cd9. Cellular and molecular life sciences : CMLS. 2011;68:3275-3292

41. $\mathrm{Xu} D$, Sharma $\mathrm{C}$, Hemler ME. Tetraspanin12 regulates adam10-dependent cleavage of amyloid precursor protein. FASEB journal : official publication of the Federation of American Societies for Experimental Biology. 2009;23:3674-3681

42. Arduise C, Abache T, Li L, Billard M, Chabanon A, Ludwig A, et al. Tetraspanins regulate adam10-mediated cleavage of tnf-alpha and epidermal growth factor. Journal of immunology. 2008;181:7002-7013

43. Bursill CA, Castro ML, Beattie DT, Nakhla S, van der Vorst E, Heather AK, et al. High-density lipoproteins suppress chemokines and chemokine receptors in vitro and in vivo. Arteriosclerosis, thrombosis, and vascular biology. 2010;30:17731778

44. Farmer JA, Liao J. Evolving concepts of the role of high-density lipoprotein in protection from atherosclerosis. Current atherosclerosis reports. 2011;13:107114

45. Sala F, Catapano AL, Norata GD. High density lipoproteins and atherosclerosis: Emerging aspects. Journal of geriatric cardiology : JGC. 2012;9:401-407

46. Badimon JJ, Badimon L, Fuster V. Regression of atherosclerotic lesions by high density lipoprotein plasma fraction in the cholesterol-fed rabbit. The Journal of clinical investigation. 1990;85:1234-1241

47. Rubin EM, Krauss RM, Spangler EA, Verstuyft JG, Clift SM. Inhibition of early atherogenesis in transgenic mice by human apolipoprotein ai. Nature. 1991;353:265-267

48. Choy EH, Panayi GS. Cytokine pathways and joint inflammation in rheumatoid arthritis. The New England journal of medicine. 2001;344:907-916

49. Janeway CA, Jr., Medzhitov R. Innate immune recognition. Annual review of immunology. 2002;20:197-216

50. Benmohamed F, Medina M, Wu YZ, Maschalidi S, Jouvion G, Guillemot L, et al. 
Toll-like receptor 9 deficiency protects mice against pseudomonas aeruginosa lung infection. PloS one. 2014;9:e90466

51. Ramphal R, Balloy V, Jyot J, Verma A, Si-Tahar M, Chignard M. Control of pseudomonas aeruginosa in the lung requires the recognition of either lipopolysaccharide or flagellin. Journal of immunology. 2008;181:586-592

52. Kanagaratham C, Camateros P, Flaczyk A, Radzioch D. Polymorphisms in toll-like receptor genes and their roles in allergic asthma and atopy. Recent patents on inflammation \& allergy drug discovery. 2011;5:45-56

53. Schepers A, Eefting D, Bonta PI, Grimbergen JM, de Vries MR, van Weel V, et al. Anti-mcp-1 gene therapy inhibits vascular smooth muscle cells proliferation and attenuates vein graft thickening both in vitro and in vivo. Arteriosclerosis, thrombosis, and vascular biology. 2006;26:2063-2069

54. Shimizu K, Minami M, Shubiki R, Lopez-Ilasaca M, MacFarlane L, Asami Y, et al. Cc chemokine receptor-1 activates intimal smooth muscle-like cells in graft arterial disease. Circulation. 2009;120:1800-1813

55. Topakian R, Sonnberger M, Nussbaumer K, Haring HP, Trenkler J, Aichner FT. Postprocedural high-density lipoprotein cholesterol predicts carotid stent patency at 1 year. European journal of neurology : the official journal of the European Federation of Neurological Societies. 2008;15:179-184

56. De Nardo D, Labzin LI, Kono H, Seki R, Schmidt SV, Beyer M, et al. High-density lipoprotein mediates anti-inflammatory reprogramming of macrophages via the transcriptional regulator atf3. Nature immunology. 2014;15:152-160

57. Kang JH, Sung MK, Kawada T, Yoo H, Kim YK, Kim JS, et al. Soybean saponins suppress the release of proinflammatory mediators by Ips-stimulated peritoneal macrophages. Cancer letters. 2005;230:219-227

58. Rye KA, Barter PJ. Cardioprotective functions of hdls. Journal of lipid research. 2014;55:168-179

59. Gordon T, Castelli WP, Hjortland MC, Kannel WB, Dawber TR. High density lipoprotein as a protective factor against coronary heart disease. The framingham study. The American journal of medicine. 1977;62:707-714

60. Tariq SM, Sidhu MS, Toth PP, Boden WE. Hdl hypothesis: Where do we stand now? Current atherosclerosis reports. 2014;16:398

61. Vergeer M, Holleboom AG, Kastelein JJ, Kuivenhoven JA. The hdl hypothesis: Does high-density lipoprotein protect from atherosclerosis? Journal of lipid research. 2010;51:2058-2073

62. Barter PJ, Brewer HB, Jr., Chapman MJ, Hennekens CH, Rader DJ, Tall AR. Cholesteryl ester transfer protein: A novel target for raising hdl and inhibiting atherosclerosis. Arteriosclerosis, thrombosis, and vascular biology. 2003;23:160167 


\section{Summary}


Cardiovascular diseases (CVDs), mostly cerebrovascular (e.g. stroke) and coronary artery disease (i.e. myocardial infarction) are the leading cause of death worldwide. The main underlying cause of CVD is atherosclerosis, a chronic inflammatory disease of mostly medium and large-sized arteries. Atherosclerotic lesions form by the accumulation of lipids, immune cells and cell debris in the vessel wall. These lesions can grow over time and eventually obstruct the blood flow, or more often these lesions rupture leading to thrombus formation that can form an occlusion. This obstruction will result in ischemic areas in downstream tissues, most commonly resulting in myocardial infarction or stroke. Although in recent decades a lot of research has been performed in this area, there is still no available therapy to fully cure or reverse atherosclerosis. This thesis focussed on the regulation of inflammation, a crucial driver of atherosclerosis, by the modulation of plasma lipid profiles, more specifically high density lipoproteins (HDL), and protein post-translational modifications mediated by members of the A Disintegrin And Metalloprotease (ADAMs) family.

In chapter 2, the role of ADAMs in angiogenesis, inflammation and atherosclerosis was reviewed. ADAMs are membrane bound proteins that can shed or cleave, among others, various inflammatory molecules like cytokines, chemokines and adhesion molecules. Some regulatory mechanisms, like lipid raft disruption, have already been described for ADAMs. However, further elucidation of this regulation, substrate specificity, redundancy and distinct functions of ADAM proteases is still warranted. For many of the ADAM proteins, associations have already been shown between ADAM expression and atherosclerotic plaque progression, mainly in humans. However, the exact causal role of ADAMs in atherosclerosis development remained elusive.

In chapter 3, we investigated the causal role of ADAM8 in atherogenesis, more specifically the role of hematopoietic ADAM8. It was already shown that polymorphisms in the ADAM8 gene in humans resulted in a significantly greater risk of myocardial infarction, associating ADAM8 to atherosclerosis. We confirmed this association by showing increased ADAM8 expression upon human lesion progression, especially in foamy macrophages. To study the specific effects of hematopoietic ADAM8 in atherosclerosis, we transferred bone marrow from ADAM8 deficient or wildtype mice into atherogenic $\mathrm{LDLr}^{-/}$mice. Surprisingly, after Western type diet feeding, we could not observe any differences in atherosclerotic lesion size or composition between wildtype 
and ADAM8 deficient animals. These unexpected results might be explained by the fact that we used a hematopoietic specific deficiency model, especially since ADAM8, in contrast to other ADAMs, physiologically exists in two active forms, being the classical membrane bound and a unique soluble form. Still a lot is unknown with regard to this soluble form. Conceivably, in atherosclerosis soluble ADAM8 secreted by other vascular cells, like endothelial and smooth muscle cells, could have compensated for the loss of ADAM8 on hematopoietic cells. Therefore, further research using total body ADAM8 deficient mice is necessary to fully unravel the potential causal role of ADAM8 in atherogenesis.

For ADAM10, we previously also showed an association of ADAM10 expression with human atherosclerotic plaque development. In chapter $\mathbf{4}$, we determined the causal role of myeloid ADAM10 in atherosclerosis using atherogenic $\mathrm{LDLr}^{-/}$mice transplanted with bone marrow from conditional knockout mice lacking ADAM10 in the myeloid lineage. After Western type diet feeding, no differences in plaque size could be observed between ADAM10 wildtype or deficient mice. However, plaque collagen content was increased in mice lacking myeloid ADAM10. Collagen is crucial for fibrous cap strength and thereby the stability of atherosclerosis lesions and can be degraded by matrix metalloproteases (MMPs), among others. In ADAM10deficient bone marrow derived macrophages, MMP-9, -13 expression and MMP-2 gelatinase activity were significantly reduced, suggesting that myeloid ADAM10 may play a causal role in modulating plaque stability. In vitro transmigration experiments showed that ADAM10-deficient macrophages have reduced migration capacity toward monocyte chemoattractant protein-1 and transmigration through collagen. Together, these results suggest that myeloid ADAM10 is crucial in inflammatory processes, like leukocyte recruitment and extracellular matrix degradation in atherosclerotic lesion, thereby possibly modulating lesion stability.

Besides myeloid cells, also endothelial cells play a crucial role in atherosclerosis. Endothelial cells form the barrier between the lumen and vessel wall, secrete many chemokines capable of attracting immune cells and express adhesion molecules, which can then bind these cells. These characteristics make endothelial cells a central player in atherogenesis. In chapter 5, we performed a proteomic analysis of conditioned medium from endothelial cells with or without ADAM10 inhibition, indicating a role for ADAM10 in transmembrane protein shedding, like chemokines and adhesion molecules. To establish the causal role of endothelial ADAM10 
in atherosclerosis, we injected endothelial cell specific ADAM10 deficient mice with an adeno-associated virus overexpressing human proprotein convertase subtilisin/kexin type 9 (PCSK9), leading to degradation of LDL receptors, rendering these mice prone for atherosclerosis development when fed a Western type diet. Contrary to our expectation, endothelial ADAM10 deficiency resulted in a marked increase in atherosclerotic lesion area, while no differences were observed in plasma cholesterol or circulating leukocyte populations. Lesions from ADAM10 deficient mice additionally showed a more advanced phenotype, characterized by more plaque necrosis and relatively less macrophages. In vivo multiphoton laser scanning microscopy imaging indicated an increase in leukocyte adhesion and transmigration in endothelial ADAM10 deficient mice. Interestingly, in line with our proteomic data, we also observed a significant reduction of plasma Annexin A5 in endothelial deficient ADAM10 mice. It has recently already been shown that Annexin A5 administration in mice resulted in a reduced lesion size and plaque macrophage content, accompanied by reduced leukocyte recruitment. This makes it very conceivable that the marked reduction in plasma Annexin A5 in our study plays a role in the observed effects on atherogenesis. However, further research will be needed to confirm this interaction and mechanism.

Besides the post-translational modifications mediated by ADAMs, we also investigated the regulation of inflammation by HDL. Various in vitro studies already showed that HDL is anti-inflammatory in endothelial cells, but did not investigate the effects of HDL on other vascular cells like smooth muscle cells (SMCs) and macrophages. In chapter 6, we investigated the effects of HDL on SMCs. Pre-incubation of SMCs with HDL, prior to TNF- $\alpha$ stimulation, significantly inhibited the secretion of CCL2, CCL5 and CX ${ }_{3} \mathrm{CL} 1$ chemokines and expression of the chemokine receptors CCR2 and $\mathrm{CX}_{3} \mathrm{CR} 1$. This decreased chemokine secretion was mediated via the PI3K - Akt - NF-KB pathway, as HDL pre-incubation inhibited phosphorylation and thus activation of all these signaling molecules. Besides these anti-inflammatory effects on chemokine secretion, we also observed that HDL strikingly reduced SMC proliferation, which appeared to be mediated via ERK signaling. Finally, we identified the scavenger receptor SR-B1 as essential membrane molecule, necessary for mediating the effects of HDL on chemokine secretion and SMC proliferation. These results are of great importance with respect to current atherosclerosis therapy, as percutaneous coronary interventions, like stent implantation, are common interventions for atherosclerosis patients but often result in excessive neointimal hyperplasia causing restenosis and early stent 
failure. Inhibiting SMC inflammation and in particular proliferation might be beneficial in this respect.

Chapter 7 focuses on the effects of HDL on macrophages. In controst to the expected anti-inflammatory effects, we surprisingly observed major proinflammatory effects of HDL on mouse as well as human macrophages in vitro, leading to increased TNF- $\alpha$ and IL-12 secretion, while reducing IL-10 release. In vivo pre-incubation of macrophages with high levels of HDL, in ApoA-I transgenic mice, also resulted in a more pro-inflammatory phenotype of macrophages from these mice, compared to wildtype mice. Using macrophages from specific cholesterol transporter (ABCA1 and ABCG1) and scavenger receptor (SR-B1 and CD36) knockout mice, we showed that none of these classical transporters were mediating these inflammatory effects. However, HDL appeared to extract membrane cholesterol via a passive manner from macrophages. Cholesterol depletion, resulting in lipid raft disruption can have major influences on intracellular signaling. Therefore, we determined the effect of HDL on various signaling pathways. We showed that NF-kB, in particular p65, is activated upon HDL incubation, although none of the described upstream activation pathways seemed to be involved in this process. This indicates that HDL results in direct phosphorylation and thus activation of $\mathrm{p} 65$. This direct phosphorylation may be mediated by protein kinase $\mathrm{C}$, which was also essential for the observed inflammatory effects. Additionally we showed a role for STAT1 signaling in mediating IL12 and IL-10 release and for ADAM proteases in mediating TNF- $\alpha$ release. These results underline the importance of a better understanding of cell-type specific effects of HDL, especially in light of to the disappointing outcome of recent clinical trials.

Finally, in chapter 8 the most important results of this thesis were discussed. The cell-type specific effects described in this thesis are of particular interest for the development of targeted therapeutic approaches, thereby modulating the clinical outcome in a disease-related and personalized manner. However, for ADAM targeting, future research must first further elucidate the precise regulation, specificity and redundancy of ADAMs in vivo. Concerning HDLbased therapies, it will be very important to focus future research on the effects of specific HDL subtypes and HDL constituents. Taken together, this thesis showed various cell-type specific effects of ADAMs and HDL on vascular inflammation. 
224 


\section{Samenvatting}


Hart- en vaatziekten, waaronder cerebrovasculaire (bijvoorbeeld beroerte) en kransslagader aandoeningen (hartinfarct) zijn wereldwijd de belangrijkste doodsoorzaken. De belangrijkste oorzaak van hart- en vaatziekten is atherosclerose oftewel aderverkalking, een chronische ontstekingsziekte van met name de middelgrote en grote slagaders. Atherosclerotische laesies worden gevormd door de opeenstapeling van lipiden, immuun cellen en cel resten in de vaatwand. Deze laesies kunnen gedurende de tijd groeien en uiteindelijk de bloedstroom belemmeren. Het gebeurt echter vaker dat deze laesies scheuren waardoor een bloedstolsel gevormd wordt dat het bloedvat (geheel of gedeeltelijk) afsluit. Deze obstructie leidt in stroomafwaarts gelegen weefsels tot een zuurstof tekort, meestal resulterend in een hartinfarct of beroerte. Hoewel de laatste decennia veel onderzoek is uitgevoerd naar atherosclerose, is er nog steeds geen therapie beschikbaar om het volledig te genezen. Dit proefschrift richt zich op de regulatie van ontsteking, een cruciale motor van atherosclerose, door het moduleren van plasma lipidenprofielen, meer in het bijzonder high density lipoproteins (HDL, ook wel bekend als het goede cholesterol) en eiwit modificaties gemedieerd door A Disintegrin And Metalloprotease (ADAMs) enzymen.

Hoofdstuk 2 geeft een overzicht van de rol van ADAMs in de vorming van nieuwe vaten, ontsteking en atherosclerose. ADAMs zijn membraangebonden eiwitten die onder andere diverse ontstekingsmoleculen zoals cytokinen, chemokinen en adhesiemoleculen van het membraan af kunnen knippen. Enkele regulerende mechanismen, zoals verstoring van cholesterol rijke gebieden in het membraan, zijn reeds beschreven voor ADAMs. Echter meer onderzoek naar deze regulatie, maar ook naar substraat specificiteit, compensatie door andere ADAMs en verschillende functies van ADAM proteasen is noodzakelijk. Voor veel van de ADAM eiwitten zijn reeds associaties aangetoond tussen hun aanwezigheid en humane atherosclerotische laesie progressie. Echter, de exacte causale rol van ADAMs in atherosclerose ontwikkeling was tot dusverre onbekend.

Hoofdstuk 3 beschrijft de causale rol van ADAM8 in atherosclerose, meer in het bijzonder de rol van ADAM8 aanwezig op bloedcellen. Er werd reeds aangetoond dat afwijkingen in het ADAM8-gen bij de mens tot een significant groter risico op een hartinfarct leiden, wat ADAM8 associeert met atherosclerose ontwikkeling. Wij bevestigden deze associatie door aan te tonen dat ADAM8 verhoogd aanwezig is tijdens humane atherosclerotische laesie progressie, voornamelijk in vet geladen ontstekingscellen, de 
schuimcellen. Om de specifieke gevolgen van bloedcel ADAM8 in atherosclerose te bestuderen, hebben we beenmerg van ADAM8 deficiënte of controle muizen overgebracht naar LDLr deficiënte muizen, die gevoelig zijn voor het ontwikkelen van atherosclerose. $\mathrm{Na}$ het geven van een vetrijk dieet konden wij geen verschil in atherosclerotische laesie grootte of samenstelling tussen controle en ADAM8 deficiënte dieren observeren. Deze onverwachte resultaten kunnen mogelijk verklaard worden doordat we gebruik gemaakt hebben van een specifiek bloedcel deficiëntie model, met name omdat ADAM8, in tegenstelling tot andere ADAMs, voorkomt in twee actieve vormen, de klassieke membraangebonden vorm en een unieke uitgescheiden vorm, waarvan nog steeds veel onbekend is. Het kan daarom zijn dat de ADAM8 uitgescheiden door andere vaatwandcellen zoals endotheelcellen en gladde spiercellen, het verlies van ADAM8 op bloedcellen heeft gecompenseerd. Daarom is verder onderzoek met behulp van volledig ADAM8 deficiënte muizen nodig om de mogelijke causale rol van ADAM8 in atherosclerose volledig te ontrafelen.

Voor ADAM10 hebben we in een eerdere studie ook een associatie van ADAM10 aanwezigheid met humane atherosclerotische plaque ontwikkeling aangetoond. In hoofdstuk 4 hebben we de causale rol van ADAM10 in bepaalde ontstekingscellen (myeloïde cellen) bij atherosclerose bepaald door gebruik te maken van LDLr deficiënte muizen welke getransplanteerd zijn met beenmerg van muizen waarbij ADAM10 specifiek in deze ontstekingscellen ontbreekt. $\mathrm{Na}$ het geven van een vetrijk dieet, kon geen verschil in laesie grootte worden waargenomen tussen ADAM10 deficiënte of controle muizen. Echter, bij de muizen zonder myeloïde ADAM10 was meer collageen aanwezig in de laesies. Collageen is essentieel voor de sterkte van de fibreuze kap en daarmee ook de stabiliteit van atherosclerotische laesies. Collageen kan onder andere afgebroken worden door matrix metalloproteasen (MMPs). In ADAM10-deficiënte beenmerg macrofagen was de MMP-9, -13 expressie en MMP-2 activiteit aanzienlijk verminderd, erop wijzend dat myeloïde ADAM10 een causale rol kan spelen bij het moduleren van de stabiliteit van laesies. In vitro transmigratie experimenten hebben aangetoond dat ADAM10-deficiënte macrofagen een verminderde migratie richting monocyte chemoattractant protein 1 en transmigratie door collageen vertonen. Samen wijzen deze resultaten erop dat myeloïde ADAM10 cruciaal is bij ontstekingsprocessen waaronder de aantrekking van ontstekingscellen en extracellulaire matrix afbraak in atherosclerotische laesies en daarmee mogelijk de stabiliteit van deze laesies beïnvloedt. 
Naast myeloïde cellen spelen ook endotheelcellen een cruciale rol bij atherosclerose. Endotheelcellen vormen de barrière tussen het lumen en de vaatwand. Eigenschappen zoals chemokine productie die immuun cellen aantrekken en de expressie van adhesiemoleculen die vervolgens deze cellen kunnen binden, maken endotheelcellen een belangrijke speler in atherosclerose. In hoofdstuk 5 hebben we een eiwit analyse, oftewel proteomics, van geconditioneerd medium van endotheelcellen met of zonder ADAM10 inhibitie uitgevoerd. De resultaten suggereren dat endotheelcel ADAM10 een belangrijke rol speelt in het knippen van membraaneiwitten, zoals chemokines en adhesiemoleculen. Om de causale rol van endotheelcel ADAM10 in atherosclerose te bepalen hebben wij endotheelcel specifieke ADAM10 deficiënte muizen geïnjecteerd met een adeno-geassocieerd virus dat humaan proprotein convertase subtilisin/kexin type 9 (PCSK9) tot overexpressie brengt, hetgeen leidt tot afbraak van LDL receptoren waardoor deze muizen gevoelig worden voor de ontwikkeling van atherosclerose bij het geven van een vetrijk dieet. In tegenstelling tot onze verwachting resulteerde endotheelcel ADAM10 deficiëntie in een duidelijke toename van atherosclerose, terwijl er geen verschillen waargenomen werden in plasma cholesterol of circulerende leukocyt populaties. Laesies van ADAM10 deficiënte muizen waren bovendien verder gevorderd, gekenmerkt door meer necrose en relatief minder macrofagen. In vivo multi-foton laser scanning microscopische beeldvorming liet een verhoging van ontstekingscel adhesie en transmigratie zien in endotheelcel ADAM10 deficiënte muizen. Opvallend is dat we in lijn met onze proteomics resultaten, ook een significante reductie van plasma Annexine A5 in endotheelcel deficiënte ADAM10 muizen zagen. Onlangs is reeds aangetoond dat Annexine A5 toediening in muizen resulteerde in een verminderde omvang van de laesie en minder macrofagen in deze laesie, gepaard gaande met een verlaging van het aantal aangetrokken ontstekingscellen. Dit maakt het aannemelijk dat de duidelijke vermindering in plasma Annexine A5 in onze studie een rol speelt bij de waargenomen effecten op atherosclerose ontwikkeling. Verder onderzoek is echter nodig om deze interactie en mechanisme te bevestigen.

Naast de eiwit modificaties gemedieerd door ADAMs, onderzochten wij ook de regulatie van ontsteking door HDL. Verschillende in vitro studies hebben reeds aangetoond dat HDL ontstekingsremmend werkt in endotheelcellen, maar de effecten van HDL op andere vasculaire cellen zoals de gladde spiercellen en macrofagen waren nog niet onderzocht. 
In hoofdstuk 6 onderzochten wij de effecten van HDL op gladde spiercellen. Pre-incubatie van gladde spiercellen met HDL alvorens TNF- $\alpha$ stimulatie remde de uitscheiding van de chemokines CCL2, CCL5 en $\mathrm{CX}_{3} \mathrm{CL} 1$ en de aanwezigheid van de chemokine receptoren $\mathrm{CCR} 2$ en $\mathrm{CX}_{3} \mathrm{CR} 1$. Deze verminderde chemokine uitscheiding was bewerkstelligd door de PI3K - Akt NF-KB route, omdat HDL pre-incubatie de fosforylering en derhalve activatie van deze signaalmoleculen remde. Naast deze anti-inflammatoire effecten op chemokine uitscheiding observeerden wij ook dat HDL de celdeling van gladde spiercellen verminderde. Dit opmerkelijke effect bleek gemedieerd te zijn door ERK signalering. Tenslotte identificeerden wij de scavenger receptor SR-B1 als essentieel membraanmolecuul, noodzakelijk voor het bewerkstelligen van de effecten van HDL op chemokine uitscheiding en celdeling van gladde spiercellen. Deze bevindingen zijn van grote waarde met betrekking tot de huidige atherosclerose therapie. Momenteel worden kransslagader interventies, zoals stentimplantaties, veel gebruikt bij atherosclerose patiënten. Echter resulteren deze interventies vaak in overmatige neointima hyperplasie (gladde spiercel groei) waardoor restenose en vroeg stent falen veroorzaakt wordt. Het remmen van gladde spiercel ontsteking en in het bijzonder de celdeling kan in dit opzicht gunstig werken.

Hoofdstuk 7 richt zich op de effecten van HDL op macrofagen. In tegenstelling tot het verwachte anti-inflammatoire effect, hebben we verrassenderwijs in vitro pro-inflammatoire effecten van HDL op zowel muizen als humane macrofagen waargenomen, resulterende in verhoogde TNF- $\alpha$ en IL-12 uitscheiding, terwijl IL-10 uitscheiding verminderd was. In vivo pre-incubatie van macrofagen met hoge niveaus van HDL in ApoA-I transgene muizen resulteerde ook in een meer pro-inflammatoir fenotype van macrofagen uit deze muizen vergeleken met controle muizen. Met behulp van macrofagen uit specifieke cholesterol transporter (ABCA1 en ABCG1) en scavenger receptor (SR-B1 en CD36) deficiënte muizen, toonden we aan dat geen van deze klassieke transporters betrokken waren bij deze inflammatoire effecten. Echter bleek HDL membraancholesterol via een passieve wijze te extraheren uit macrofagen. De verstoring van cholesterolrijke gebieden in het membraan door cholesterol onttrekking kan grote invloed hebben op intracellulaire signaaloverdracht. Derhalve hebben we het effect van HDL op verschillende signaalroutes bepaald. NF-KB, in het bijzonder p65, werd geactiveerd door HDL incubatie, hoewel geen van de beschreven activeringsroutes stroomopwaarts betrokken leken bij dit proces, wat aangeeft dat HDL leidt tot directe fosforylering en derhalve activering van p65. Deze fosforylering 
kan worden gemedieerd door proteïne kinase $C$, die ook essentieel bleek te zijn voor de waargenomen inflammatoire effecten. Daarnaast toonden wij een rol aan voor STAT1 signalering bij het mediëren van IL-12 en IL10 secretie en ADAM proteasen bij het mediëren van TNF- $\alpha$ secretie. Deze resultaten accentueren de urgentie van het verkrijgen van meer inzicht in de cel specifieke effecten van HDL.

Tenslotte werden in hoofdstuk 8 de belangrijkste resultaten van dit proefschrift besproken en in perspectief tot andere literatuur geplaatst. De cel specifieke effecten beschreven in dit proefschrift zijn in het bijzonder van belang voor de ontwikkeling van doelgerichte therapieën, waardoor de klinische resultaten in een ziektegerelateerde en patiënt specifieke manier gemoduleerd kunnen worden. Voor therapie gericht op ADAMs is toekomstig onderzoek, waar de precieze regulatie, specificiteit en compensatie door andere ADAMs wordt ontrafelt in vivo, een vereiste. Voor HDL gebaseerde therapieën zal het zeer belangrijk zijn om toekomstig onderzoek te richten op de effecten van specifieke subtypen HDL en HDL bestanddelen. Tezamen laten de resultaten in dit proefschrift diverse cel specifieke effecten van ADAMs en HDL op vasculaire ontsteking zien. 


\section{Valorisation}


After years or even decades of research, cardiovascular disease (CVD) still remains the main cause of death worldwide, accounting for 16.7 million deaths annually ${ }^{1,2}$. However, not all patients with CVD die, but are often hospitalized or should receive lifelong treatment, leading to high healthcare costs. This economic burden can be quite substantial, since in the United States alone, the cost related to CVD were more than $\$ 312$ billion per year ${ }^{3}$. In addition, also the social burden on the patients but also on their environment should not be underestimated. The main underlying pathology of CVD is atherosclerosis, a chronic inflammatory disease of mainly medium and large sized arteries. In an effort to reduce the high mortality number and the burden caused by CVD, extensive research into the underlying pathology is essential. Fundamental research can contribute to a better understanding of this complex disease, and thereby create new possibilities to target and treat this pathology.

In this thesis, we investigated the cell-type specific role of ADAMs in atherosclerosis, more specifically ADAM8 and ADAM10. The ultimate goal of these studies is the identification of possible therapeutic targets to combat atherosclerosis. It has already been suggested that ADAM inhibition could be efficient for treating various other diseases. Looking at ADAM10 and its closely related family member ADAM17, selective inhibition of these proteases has already been shown to have anti-cancer effects and clinical trials are currently being performed ${ }^{4}$. Inhibitors for ADAM17 have also been shown to be effective against arthritis in animal models ${ }^{5}$, however clinical trials failed to confirm this positive outcome ${ }^{6}$. Although ADAM10 and ADAM17, substrate-wise look like the most suited candidates for targeting, caution is warranted. One of the main targets of ADAMs involved in growth and development are ligands of epidermal growth factor receptors (EGFRs) 7. Therefore, one should be careful with inhibiting ADAMs, to avoid unwanted side effects on cell growth for example.

This is further supported by results from chapters 4 and 5 from this thesis. In chapter 4, we showed that myeloid specific ADAM10 inhibition does not affect lesion size, but rather shifts the phenotype of the lesions to a more fibrotic one. Thereby myeloid ADAM10 inhibition might increase plaque stability, which would be beneficial for clinical outcome. However, in chapter 5, endothelial specific ADAM10 deletion results in a significant increase in lesion size, which would of course be detrimental for clinical outcome. These results clearly indicate that cell-type specific ADAM10 inhibition might be on 
the one hand beneficial or on the other hand detrimental for disease outcome, depending on the targeted cell subset. Unfortunately, to date the developed inhibitors for ADAMs are not yet cell-type restricted nor ADAM specific, therefore a future focus must lie on developing more specific intervention approaches. Recently, an alternative method of specifically inhibiting ADAMs was proposed, namely inhibition of $\mathrm{RHOM} 2^{8}$. This inactive member of the Rhomboid intramembrane proteinase family regulates the maturation of ADAM17, but is only expressed in hematopoietic cells. Using a siRNAapproach it was shown that inhibition of $\mathrm{R}$ ROM 2 resulted in decreased furin-mediated maturation and trafficking of ADAM17 to the cell surface in both mouse and human monocytes. Therefore iRHOM2 targeting might be an attractive new, cell-type specific method of blocking ADAM17. Further research is needed to identify comparable regulators of other ADAMs. Therefore cell-type specific inhibition seems essential to overcome this hurdle.

Another therapeutic option is to specifically target the disintegrin domain of ADAMs, which for some ADAMs already has been shown to be responsible for integrin binding. Thereby influencing various processes like cell adhesion, migration and proliferation. The inhibitory effect might be more specific but probably also much less effective, since only a limited amount of ADAMs function is inhibited. For ADAM10 and ADAM17 the role of this domain has not yet been elucidated. Therefore future research is first needed to elucidate this precise role, before evaluating the therapeutic potential and risk.

Besides therapeutic targets, ADAMs could also serve as diagnostic as well as prognostic biomarkers. Expression levels of ADAM8, 10 and 17 correlated with atherosclerotic lesion progression. Additionally, ADAM8 polymorphisms associate ADAM8 with risk of myocardial infarction. Untill now research into ADAMs as biomarkers has been limited, especially in the field of atherosclerosis and CVDs. However, several reports do suggest that a number of ADAMs may at least be used as cancer biomarker ${ }^{4}$. For example, for ADAM12 it has been shown that high urinary ADAM12 levels were correlated with the presence of breast cancer. As well as breast cancer, urinary ADAM12 was also elevated in patients with bladder cancer and ADAM12 measurement even showed to be more sensitive than the currently used standard cytology. As a more prognostic marker, high levels of ADAM17 were found to predict adverse outcome in patients with breast cancer. These data clearly show prospects for ADAMs as biomarkers, although for cardiovascular diseases this remains to be established. 
Besides modulation of ADAMs as approach to dampen vascular inflammation, we also focused on modulating lipoproteins in this thesis, in particular HDL. Various clinical and epidemiological studies showed that HDL levels inversely correlate with CVD risk ${ }^{9}$. However, many of the recently performed and described clinical trials using various methods of increasing HDL levels in humans did not show any effect on cardiovascular event rates. Especially the meta-analysis of 39 clinical trials showing that niacin, fibrates and CETP inhibitors did not reduce all-cause mortality or the incidence of $\mathrm{MI}$ or stroke ${ }^{10}$, fuelled a lively debate about the effectiveness of HDL raising therapeutic strategies.

In this thesis, we showed strikingly divergent, cell-type effects of HDL on inflammation, showing anti-inflammatory effects in smooth muscle cells (chapter 6), but pro-inflammatory effects in macrophages (chapter 7). Therefore, therapies using HDL should be used in a personalized and more importantly, disease specific manner. For example our observation that HDL has inhibiting effects on smooth muscle cell proliferation may be beneficial for neointimal hyperplasia, but detrimental for other pathologies. Additionally, the pro-inflammatory HDL effects on macrophages may be beneficial for bacterial clearance for example, but detrimental for patients with rheumatoid arthritis. Besides this pathology specific approach, future therapeutic research should also focus on the specific composition of HDL. HDLs are very heterogeneous, consisting of many different subpopulations. Especially the effects of specific subtypes are still relatively limitedly studied. HDL raising therapies have varying effects on these different subpopulations ${ }^{11}$, making further knowledge of the differences between these populations warranted. This is supported by comparing results from chapter 7 with a recently published study by De Nardo et al. ${ }^{12}$, where they observe antiinflammatory effects on macrophages using HDL composed of soy-bean derived phospholipids, suggesting that the phospholipid composition of HDL may influence the outcome of HDL treatment. Therefore, next to the celltype and disease specific approach, it is necessary to further elucidate the exact relation between observed HDL effects and HDL composition. This will be essential to continue developing more specific and targeted HDL-based approaches to combat disease. 


\section{References}

1. Dahlof B. Cardiovascular disease risk factors: Epidemiology and risk assessment. The American journal of cardiology. 2010;105:3A-9A

2. Lloyd-Jones DM. Cardiovascular risk prediction: Basic concepts, current status, and future directions. Circulation. 2010;121:1768-1777

3. Go AS, American Heart Association statistics committee and stroke statistics subcommitte. Executive summary: heart disease and stroke statistics - 2013 update: a report from the americam heart association. Circulation. 2013;127:143152

4. Duffy MJ, Mullooly M, O'Donovan N, Sukor S, Crown J, Pierce A, et al. The adams family of proteases: New biomarkers and therapeutic targets for cancer? Clinical proteomics. 2011;8:9

5. Arribas J, Esselens C. Adam17 as a therapeutic target in multiple diseases. Current pharmaceutical design. 2009;15:2319-2335

6. Moss ML, Sklair-Tavron L, Nudelman R. Drug insight: Tumor necrosis factorconverting enzyme as a pharmaceutical target for rheumatoid arthritis. Nature clinical practice. Rheumatology. 2008;4:300-309

7. Sahin U, Weskamp G, Kelly K, Zhou HM, Higashiyama S, et al. Distinct roles for ADAM10 and ADAM17 in ectodomain shedding of six EGFR ligands. J Cell Biol. 2004;164(5):769-779

8. Issuree PD, Maretzky T, Mcllwain DR, Monette S, Qing X, Lang PA, et al. iRHOM2 is a critical pathogenic mediator of inflammatory arthritis. J Clin Invest. 2013;123(2):928-932

9. Emerging Risk Factors C, Di Angelantonio E, Sarwar N, Perry P, Kaptoge S, Ray $\mathrm{KK}$, et al. Major lipids, apolipoproteins, and risk of vascular disease. Jama. 2009;302:1993-2000

10. Keene D, Price C, Shun-Shin MJ, Francis DP. Effect on cardiovascular risk of high density lipoprotein targeted drug treatments niacin, fibrates, and cetp inhibitors: Meta-analysis of randomised controlled trials including 117,411 patients. Bmj. 2014;349:g4379

11. Rye KA, Barter PJ. Cardioprotective functions of hdls. Journal of lipid research. 2014;55:168-179

12. De Nardo D, Labzin LI, Kono H, Seki R, Schmidt SV, Beyer M, et al. High-density lipoprotein mediates anti-inflammatory reprogramming of macrophages via the transcriptional regulator atf3. Nature immunology. 2014;15:152-160 
236 


\section{Acknowledgements Dankwoord}


Zoals de meeste zullen weten ben ik iemand van weinig woorden, maar omdat dit waarschijnlijk voor de meeste de eerste en de enige woorden zijn die jullie uit dit boekje zullen lezen ga ik proberen er iets moois van te maken. In dit laatste hoofdstuk wil ik heel graag alle mensen bedanken die mij de afgelopen jaren hebben geholpen / gesteund en daarmee dit boekje mede mogelijk hebben gemaakt.

Allereerst wil ik natuurlijk mijn gehele promotieteam bedanken.

Beste dr. Donners, beste Marjo, tijdens al die jaren stond jouw deur altijd voor mij open en kon ik bij jou terecht voor hulp, input of om te discussiëren over zaken. Ook toen ik bij je kwam met een niet-ADAM, maar lipoproteïne project heb je je nog verder in die wereld verdiept en konden we samen altijd erg goede discussies voeren over de experimenten en vooral de verrassende resultaten. Ik wil je dan ook oprecht heel erg hartelijk bedanken voor alle hulp, steun en betrokkenheid al die jaren!!

Beste Prof. dr. Biessen, beste Erik, ik kan me nog heel goed mijn eerste werkbespreking met jou herinneren. Na 2 uur bespreken, kwam ik met een hele waslijst aan nieuwe ideeën, experimentele plannen en zelfs met nieuwe 4 jarige AIO-projecten naar buiten. De werkbesprekingen waren dan ook altijd uitermate motiverend en het was altijd heel verhelderend om met jou te discussiëren. Heel graag wil ik je bedanken voor de leerzame tijd binnen jouw groep. Bedankt voor alle motivatie en onvergetelijke speeches!

Beste Prof. dr. De Winther, beste Menno, binnen MolGen maakte ik deel uit van je Maastrichtse macrofaag-club, een goed geoliede machine, vol gezelligheid. De werkbesprekingen die we hadden waren ook altijd zeer productief en zeker in het begin van het HDL verhaal was jouw vastberadenheid erg motiverend. Ik wil je dan ook heel erg graag bedanken voor alle begeleiding die jij me hebt gegeven!

Beste Prof. dr. Glatz, beste Jan, bedankt voor je steun en input tijdens mijn MolGen jaren. Je vragen en suggesties bij werkbesprekingen waren altijd zeer hulpzaam.

I would like to thank the members of the judging committee, consisting of Prof. dr. Reutelingsperger (chairman), Prof. dr. Barter, Prof. dr. Daemen, Prof. dr. Schalkwijk and Prof. dr. Weber for reading and judging my thesis. 
Dan wil ik graag mijn twee paranimfen bedanken, Kosta en Marten. Allereerst Kosta, dankjewel voor de enorme hulp!! Tijdens je senior stage heb je enorm veel werk verzet voor het HDL project (onder andere..). We hebben toen heeeeeel erg veel uren samen in de kweek gezeten met mijn old-school radio. Ook als collega bij de Patho zorgde je altijd voor veel gezelligheid, met je karakteristieke lach of als je weer een idioot filmpje had gevonden. Kortom, heel erg bedankt voor alle hulp en vooral ook gezelligheid!! Dan Marten, het eerste jaar hebben we bij MolGen als collega's kunnen werken. Op het lab zorgde je met je droge humor voor heel veel gezelligheid! Daarna ging je helaas met Menno mee naar Amsterdam, maar zijn we gelukkig wel nog veel samen blijven werken. Ik wil ik je van harte bedanken voor alle hulp en steun de laatste jaren! En natuurlijk beide super bedankt dat jullie tijdens mijn promotie aan mijn zijde willen staan!

Tijdens mijn promotie-onderzoek heb ik, zeker door de verhuizing met veel collega's gewerkt. Te beginnen bij mijn eerste afdeling, Moleculaire Genetica. In het bijzonder wil ik de overige leden van de macrofaag-club hartelijk bedanken! Ronit, thank you very much for all your questions/suggestions and discussions. Nadine, het was altijd een zeer gezellige boel met jou erbij.. op het lab waar je me veel geholpen hebt, maar ook zeker in de koffiekamer! Heel erg bedankt! Ine, je stond altijd klaar om me te helpen op het lab en mee te denken over de projecten. Het was altijd heel prettig om met je samen te werken. Bedankt voor alles! Mike, hartelijk bedankt voor al je hulp met het ADAM10-BMT project (en HDL project..on the side) tijdens je stage. Natuurlijk ook voor alle gezelligheid, als mijn student. maar zeker ook daarna als collega! Tim, Sofie en Tom, bedankt voor de leuke tijd op het lab en alle nuttige input tijdens de meetings! Patrick en Chantal, hartelijk bedankt voor alle hulp op het lab en met de muizen! Daarna wil ik natuurlijk graag alle mede-AIO's, Guus, Marie, Yeliz, Yvonne, Peggy, Jieyi, Yilin en Karen en vaste krachten, Petra, Willem, Vivian, Joost, Will, Dietbert and Dipanjan, hartelijk bedanken voor alle hulp en betrokkenheid!

Dan mijn tweede afdeling, de Pathologie. Beginnend met mijn kamergenoten. Thomas, bedankt voor al je hulp op het lab en vooral met de muizen. Je was ook altijd in voor een geintje of om borrels te organiseren.. Elke, jij was minstens net zo actief betrokken bij het creëren van de gezellige sfeer op de kamer en het lab. Hiernaast werk je ook enorm hard en ben je heel gedreven. Heel veel succes beide met jullie projecten! Anke, de nieuwkomer op de kamer, maar toch een oude bekende. Je was mijn eerste student tijdens 
mijn AIO-schap, bedankt voor al je inzet en harde werken in die tijd aan het ADAMs project! Marchy, it seemed like you were always happy. I wish you all the best in finishing up your thesis! Taghi, thank you for all your support, especially the last weeks with the array analysis.

Bart, hoewel je helaas geen kamergenoot was, stond je wel altijd klaar om te helpen. Judith, Lieve, Sylvia en Jack, bedankt voor jullie interesse, kritische vragen en goede adviezen tijdens de werkbesprekingen. Anjana, Pawel, Sebastien and Chiara, thank you for your involvement and input during meetings. Mat, bedankt voor al je hulp met de BD pathway en het sorteren. Erwin, je zorgde voor veel gezelligheid op het lab en stond altijd met je telefoon-camera in de aanslag als iemand weer iets doms deed. Bedankt voor alle hulp. Anique en Clairy, hartelijk bedankt voor alle hulp op en rond het lab. Danielle, bedankt voor al je hulp met afspraken plannen en het papierwerk rond mijn promotie.

Marion, tja..ik kon niet bedenken in welke alinea ik jou kon plaatsen..daarom krijg je je eigen! Tussen al het reizen door tussen de 3 labs, krijg je enorm veel werk gedaan...ook voor mij heb je heel veel werk verzet en daarvoor wil ik je dan ook heel erg hartelijk bedanken!! Ook voor het functioneren als mijn "privé-koerier" tussen Maastricht en Amsterdam, wil ik je heel erg bedanken! Ik ben al heel lang geleden de tel kwijt geraakt, zoveel pakketjes heb jij voor me vervoerd...

Dan nog iemand, die maar moeilijk te plaatsen is.. Timo, bedankt voor al je hulp over de jaren. Je had niet voor niets altijd de bijnaam Timopedia.. voor info/hulp of wat dan ook kan men altijd bij jou terecht. Zeker ook hartelijk bedankt voor alle MP hulp, zelfs dagen achter elkaar tot na middernacht!!

Verder hebben de laatste jaren aardig wat studenten, die nog niet genoemd zijn, meegeholpen aan de projecten. Bart, Kenrick, Shannen, Anne, Marijn en Steven, bedankt voor het harde werken en jullie betrokkenheid en inzet bij de projecten en de leuke tijd tijdens jullie stages! 
Promotieonderzoek doe je nooit alleen, zelfs niet met twee labs. Daarom wil ik ook graag al mijn collaboraties van harte bedanken voor alle input en vooral hun bereidwilligheid om samen te werken bij de verschillende projecten. Beginnende binnen de UM: Chris, Leon, Dennis, Brecht, bedankt voor alle hulp en discussies over Annexine en gladde spier cellen. Marijke, bedankt voor alle inhibitors en je confocal expertise. Jogchum en Maurice, bedankt voor alle hulp bij het HDL project, zowel praktisch als theoretische discussies. Rory, bedankt voor je input bij het endotheel verhaal, jouw expertise was altijd zeer hulpzaam. Daarna de externe mensen: Miranda, hartelijk bedankt voor al je betrokkenheid en hulp bij het HDL project. Het was heel fijn om met jou samen te werken en te discussiëren over het project op congressen. Pieter, bedankt voor al je inbreng in het HDL verhaal en je gastvrijheid toen ik even in Marseille op bezoek was. Jammer, dat we elkaar weer net gaan mislopen in Maastricht! Sander en Leonie, bedankt voor jullie hulp bij het NF-KB gedeelte. Jaap, bedankt voor al je input en vooral dat we zo vaak nog gebruik mochten maken van Timo's expertise.

Special thanks to all the collaborators, for their support and willingness to collaborate on the different projects. Andreas and Daniela, thank you for your support and successful collaboration over the years. Toby Lawrence, Jörg Bartsch, Paul Saftig, Stefan Rose-John and Jacob Bentzon, thank you for the nice collaboration.

Dear Kerry, first of all thank you very much for coming to Maastricht to join in the corona for my defense. Furthermore, thank you for giving me the opportunity to do my final internship of my master in your lab at the HRI. Even when I left, we saw each other occasionally at conferences, were we could discuss the so surprising results of the HDL-macrophage paper. Thank you for all your scientific support over the years.

Dear Chris, I really enjoyed working for/with you during my internship. You were always really enthusiastic about new data, which was very motivating. Your good spirit and of course scientific knowledge, contributed a great deal to the nice time I had in your lab. It even led to a very nice publication, which is part of this thesis. Thank you very much for everything!

Verder wil ik alle medewerkers van de CPV die betrokken waren bij de dierverzorging en -experimenten, Richard, Saskia, Clarice, Rik, Paulien, Sytske, Anouk en Mandy graag bedanken voor de fijne samenwerking. 
Ik wil graag CARIM heel hartelijk bedanken voor het toekennen van de PhDaward aan mij, die het mogelijk heeft gemaakt dat ik dit promotieonderzoek heb uitgevoerd! Daarnaast wil ik de mensen binnen CARIM (Rob, Riet, Esther, Tara en alle leden van de EPC) graag bedanken voor de leuke en leerzame tijd die ik heb gehad als AIO-vertegenwoordiger. Natuurlijk ook dank aan de overige I'M CARIM leden (Timo, Yvonne, Siamack, Tom en Elke) voor de gezellige tijd.

Dear Christian and Yvonne, thank you very much for giving me the great opportunity to continue my scientific career in Munich. I am very honored to be working in your group and hope that we will get some nice publications together in the coming years.

Natuurlijk wil ik ook mijn vrienden bedanken...het MLW-revival vriendenclubje! Auke, Marten, Bram, Irene en Mirelle, allemaal superrrrr bedankt voor de leuke tijd die we samen hebben gehad gedurende de laatste 10 jaar!! Ik heb genoten van de vele spelletjes-avonden en SinterKerst-weekendjes. Hoewel we natuurlijk altijd wel iets over onderzoek praatte, zorgde jullie altijd voor veel ontspanning en gelach met alle gekkigheid, de Geer en Goor imitaties en de koehandel special effects. Ik hoop dat we elkaar nog veel blijven zien en natuurlijk zijn jullie allemaal Altijd van harte welkom in München, niet alleen in oktober....

Mirelle, jij bent een ontzettend grote steun voor mij!! Je weet me altijd te motiveren en op te vrolijken als het eens iets minder gaat..en we kunnen ontzettend veel plezier hebben samen! Zowel thuis, maar zeker ook op vakantie of met weekendjes weg... en dan de social deals (liefste in een sterren-restaurant) niet te vergeten! Ook van het samen wielrennen heb ik altijd erg genoten, kom je binnenkort samen alpencolletjes fietsen? En natuurlijk de concerten/festivals... poeh naar hoeveel zijn we al geweest samen... Je betekent echt enorm veel voor mij en ik geef ontzettend veel om jou!! Nu kan ik niet meer met mijn mazda naar je toe snellen, maar ik weet zeker dat de KLM / NS het druk gaan krijgen met mij de komende jaren...

Dan de familie Huijskens. Joop en Liesbeth, bedankt dat ik altijd bij jullie welkom ben..en altijd zo gastvrij wordt onthaald. Het is altijd heel gezellig en ontspannend (tuin..schouw..) om bij jullie te zijn. Bedankt voor alles! Rudolf en Inge, bedankt voor jullie betrokkenheid en interesse altijd! 
Tenslotte, de mensen die ik het aller langste ken, mijn familie. Maurice en Sanne, bedankt voor alle interesse die jullie over de jaren hebben getoond! En de ontspanning tijdens de avonden dat ik bij jullie op bezoek was! Mam en pap, heel erg bedankt voor alles over al die jaren. Bedankt dat jullie me de kans hebben gegeven om te gaan studeren en daarin altijd hebben gesteund en gemotiveerd hebben. Ook in de laatste jaren, toen het drukker en drukker werd en ik steeds minder tijd had om langs te komen. Maar ik wist dat ik altijd bij jullie terecht kon voor alles en de deur altijd open stond voor mij! Bedankt! 


\section{Curriculum Vitae}


Emiel Petrus Carla van der Vorst was born on August $14^{\text {th }}, 1987$ in Sittard, The Netherlands. He acquired his secondary school diploma in 2005 at Trevianum in Sittard. He graduated his bachelor education in Molecular Life Sciences at Maastricht University with honours in 2008 and subsequently studied the master Cardiovascular Biology and Medicine. Emiel performed his senior internship at the Heart Research Institute in Sydney, Australia, under the supervision of Prof. dr. Kerry-Anne Rye and dr. Christina Bursill. After obtaining a competitive personal grant for four years doctorate training, the CARIM PhD award, he started in 2010 with his doctoral research within CARIM, CArdiovascular Research Institute Maastricht. The project started at the department of Molecular Genetics at $\mathrm{MUMC}^{+}$under the supervision of Prof. dr. Menno de Winther, Prof. dr. Jan Glatz and dr. Marjo Donners, and was continued at the department of Pathology at $\mathrm{MUMC}^{+}$under the supervision of Prof. dr. Erik Biessen and dr. Marjo Donners. The results obtained during this period are described in this thesis. In February 2015, he graduated his master Management at the Open University of The Netherlands. In March 2015, he has started his post-doctoral research at the Institute for Cardiovascular Prevention in Munich, Germany, in the team of Prof. dr. Christian Weber and dr. Yvonne Döring. 
Emiel Petrus Carla van der Vorst werd geboren op 14 augustus 1987 te Sittard, Nederland. Hij heeft zijn VWO diploma behaald in 2005 aan de scholengemeenschap Trevianum te Sittard. Hierna is hij begonnen aan de bachelor opleiding Moleculaire Levenswetenschappen aan de Universiteit van Maastricht, waar hij in 2008 cum laude is afgestudeerd. Aansluitend is Emiel de master Cardiovascular Biology and Medicine gaan studeren, eveneens aan de Universiteit van Maastricht. Tijdens zijn afstudeerstage heeft hij onderzoek uitgevoerd in het Heart Research Institute in Sydney, Australië, onder leiding van Prof. dr. Kerry-Anne Rye en dr. Christina Bursill. $\mathrm{Na}$ het behalen van de CARIM PhD award in 2010, een competitieve persoonsgebonden beurs voor 4 jaar promotieonderzoek, is hij zijn promotietraject gestart binnen CARIM, CArdiovascular Research Institute Maastricht bij de afdeling Moleculaire Genetica en de afdeling Pathologie van het $\mathrm{MUMC}^{+}$onder leiding van Prof. dr. Menno de Winther, Prof. dr. Jan Glatz, Prof. dr. Erik Biessen en dr. Marjo Donners. De resultaten behaald tijdens dit traject zijn beschreven in dit proefschrift. In februari 2015 is hij afgestuurd voor de master Management aan de Open Universiteit Nederland. Vanaf maart 2015 verricht hij zijn postdoctoraal onderzoek aan het Institute for Cardiovascular Prevention in München, Duitsland, onder leiding van Prof. dr. Christian Weber en dr. Yvonne Döring. 


\section{List of publications}

Myeloid A Disintegrin And Metalloprotease ADAM10 deficiency enhances atherosclerotic plaque fibrosis.

Emiel P.C. van der Vorst, Mike Jeurissen, Ine M.J. Wolfs, Anke Keijbeck, Kosta Theodorou, Erwin Wijnands, Leon Schurgers, Silvio Weber, Marion J. Gijbels, Anouk A.J. Hamers, Daniela Dreymueller, Stefan Rose-John, Menno P.J. de Winther, Andreas Ludwig, Paul Saftig, ErikA.L. Biessen, Marjo M.P.C. Donners.

Am J Pathol. 2015 Apr;185(4):1145-55.

AnxA5 reduces plaque inflammation of advanced atherosclerotic lesions in apoE $\mathrm{E}^{-/}$mice.

Mathias Burgmaier, Kristof Schutters, Brecht Willems, Emiel P.C. van der Vorst, Dennis Kusters, Martijn Chatrou, Lucy Norling, Erik A.L. Biessen, Jack Cleutjens, Mauro Perretti, Leon J. Schurgers, Chris P.M. Reutelingsperger. J Cell Mol Med. 2014 Oct;18(10):2117-24.

Leukocytes require the metalloproteinase ADAM10 but not ADAM17 for efficient cell migration and for inflammatory leukocyte recruitment into the alveolar space.

Jessica Pruessmeyer, Franz Martin Hess, Henriette Ahlert, Esther Groth, Tobias Pasqualon, Nicole Schwarz, Stella Nyamoya, Jos Kollert, Emiel P.C. van der Vorst, Marjo M.P.C. Donners, Christian Martin, Stefan Uhlig, Paul Saftig, Daniela Dreymueller, Andreas Ludwig.

Blood, 2014 Jun;123(26):4077-88.

Reprogramming macrophages to an anti-inflammatory phenotype by helminth antigens reduces murine atherosclerosis.

Ine M.J. Wolfs, J. Lauran Stöger, Pieter Goossens, Chantal C.H. Pöttgens, Marion J.J. Gijbels, Erwin Wijnands, Emiel P.C. van der Vorst, Patrick van Gorp, Linda Beckers, David Engel, Erik A.L. Biessen, Georg Kraal, Irma van Die, Marjo M.P.C. Donners, Menno P.J. de Winther.

FASEB J., 2014 Jan;28(1):288-99. 
High-density lipoproteins suppress chemokine expression and proliferation in human vascular smooth muscle cells.

Emiel P.C. van der Vorst*, Laura Z. Vanags*, Louise L. Dunn, Hamish C. Prosser, Kerry-Anne Rye, Christina A. Bursill. *Shared first authorship.

FASEB J., 2013 Apr;27(4):1413-25.

A disintegrin and metalloproteases: molecular scissors in angiogenesis, inflammation and atherosclerosis.

Emiel P.C. van der Vorst ${ }^{*}$, Anke A. Keijbeck*, Menno P.J. de Winther, Marjo M.P.C. Donners. *Shared first authorship.

Atherosclerosis, 2012 Oct;224(2):302-8.

Hematopoietic miR155 deficiency enhances atherosclerosis and decreases plaque stability in hyperlipidemic mice.

Marjo M.P.C. Donners, Ine M.J. Wolfs, J. Lauran Stöger, Emiel P.C. van der Vorst, Chantal C.H. Pöttgens, Stephane Heymans, Blanche Schroen, Marion J. Gijbels, Menno P.J. de Winther.

Plos One, 2012;7(4).

High-density lipoproteins suppress chemokines and chemokine receptors in vitro and in vivo.

Christina A. Bursill, Maria L. Castro, Douglas T. Beattie, Shirley Nakhla, Emiel P.C. van der Vorst, Alison K. Heather, Philip J. Barter, Kerry-Anne Rye. ATVB, 2010 Sep;30(9):1773-8.

High density lipoproteins exert pro-inflammatory effects on macrophages via passive cholesterol depletion and PKC-dependent NF-KB/STAT1 activation.

Emiel P.C. van der Vorst, Kosta Theodorou, Marten A. Hoeksema, Pieter Goossens, Christina A. Bursill, Sander W. Tas, Ine M.J. Wolfs, Debby P.Y. Koonen, Shahla Abdollahi-Roodsaz, Toby Lawrence, Jogchum Plat, Miranda van Eck, Kerry-Anne Rye, Menno P.J. de Winther, Erik A.L. Biessen, Marjo M.P.C. Donners.

In revision at Cell Metabolism. 
Exogenous annexin A1 attenuates atherogenesis in $\mathrm{LDLR}^{-/-}$mice on Western Type Diet.

Dennis H.M. Kusters, Martijn L. Chatrou, Brecht A.G. Willems, Marijke De Saint-Hubert, Matthias Bauwens, Emiel P.C. van der Vorst, Stefania Bena, Erik A.L. Biessen, Mauro Perretti, Leon J. Schurgers, Chris P.M. Reutelingsperger.

In revision at Cardiovascular Research.

Increased adventitial lymphatic capillaries in atherosclerosis: a path for T cell trafficking?

Timo Rademakers* ${ }^{*}$ Emiel P.C. van der Vorst' ${ }^{*}$ Isabelle T.M.N Daissormont*, Jeroen J.T. Otten, Andrey Anisimov, Harri Nurmi, Marc A.M.J. van Zandvoort, Sylvia Heeneman, Kari Alitalo, Erik A.L. Biessen. *Shared first authorship. In preparation.

Hematopoietic ADAM8 deficiency does not influence atherosclerotic plaque area or composition, despite its upregulation during plaque progression.

Emiel P.C. van der Vorst, Kosta Theodorou, Thomas Theelen, Bart Smeets, Ine M.J. Wolfs, Mike Jeurissen, Marion J. Gijbels, Erwin Wijnands, Andreas Ludwig, Jörg W. Bartsch, Erik A.L. Biessen, Marjo M.P.C. Donners. In preparation.

Endothelial ADAM10 deficiency modulates leukocyte recruitment and remarkably augments atherosclerotic lesion development.

Emiel P.C. van der Vorst, Timo Rademakers, Kosta Theodorou, Marijn Brilman, Jaap van Buul, Thomas L. Theelen, Erwin Wijnands, Marion J. Gijbels, Leon Schurgers, Mat Rousch, Sofie M. Walenbergh, Michael Lehrke, Corinna Lebherz, Dominique de Kleijn, Siu Kwan Sze, Daniela Dreymueller, Jacob Bentzon, Andreas Ludwig, Erik A.L. Biessen, Marjo M.P.C. Donners. In preparation. 


\section{Oral presentations}

"High-density lipoproteins exert pro-inflammatory effects on macrophages via passive cholesterol depletion and PKC-dependent NF-kB/STAT1 activation."

- Frontiers in Cardiovascular Biology 2014, Barcelona, Spain

- Cardiovascular Conference 2014, Noordwijkerhout, The Netherlands

- MIMSA, Maastricht Inflammation in the Metabolic Syndrome and

Atherosclerosis Symposium 2013, Maastricht, The Netherlands (Invited speaker)

- CARIM symposium 2010, Maastricht, The Netherlands (Invited speaker)

\section{Poster presentations}

"High-density lipoproteins exert pro-inflammatory effects on macrophages via passive cholesterol depletion and PKC-dependent NF-kB/STAT1 activation."

- IAS 2015, Amsterdam, The Netherlands

- $13^{\text {th }}$ Dutch-German Joint Meeting of the Molecular Cardiology Groups

2015, Garmisch-Partenkirchen, Germany

- IAS workshop on HDL 2014, Rome, Italy

- Cardiovascular Conference 2013, Noordwijkerhout, The Netherlands

- Scandinavian Society for Atherosclerosis Research 2012, Humlebaek,

Denmark

- Cardiovascular Conference 2012, Noordwijkerhout, The Netherlands

"Myeloid A Disintegrin And Metalloprotease ADAM10 deficiency enhances atherosclerotic plaque fibrosis."

- Scandinavian Society for Atherosclerosis Research 2013, Humlebaek,

Denmark

- Cardiovascular Conference 2013, Noordwijkerhout, The Netherlands

- CARIM symposium 2012, Maastricht, The Netherlands

"High-density lipoproteins suppress chemokine expression and proliferation in human vascular smooth muscle cells."

- ATVB Scientific Sessions 2011, Chicago, USA 


\section{Awards and prizes}

2015: Award for Best Poster Presentation

$13^{\text {th }}$ Dutch-German Joint Meeting of the Molecular Cardiology Groups, Garmisch-Partenkirchen, Germany

"High density lipoproteins exert pro-inflammatory effects on macrophages via passive cholesterol depletion and PKC-dependent NF-kB/STAT1 activation."

2014: Scholarship of the Professors' fund / Limburg University Fund 2014 Personal grant for additional funding for exceptional young investigators

2014: Award for Best Oral Presentation

Cardiovascular Conference, Noordwijkerhout, The Netherlands

"High density lipoproteins exert pro-inflammatory effect on macrophages via NF-kB activation and passive cholesterol efflux."

2013: Award for Best Poster Presentation

Cardiovascular Conference, Noordwijkerhout, The Netherlands "High density lipoproteins exert pro-inflammatory effect on macrophages via NF-kB activation and passive cholesterol efflux."

2010: Young Investigator Award for accepted Abstract

Australian Atherosclerosis Society and Asia Pacific Society of Atherosclerosis and Vascular Disease joint meeting, Cairns, Australia "High-Density Lipoproteins Suppress Chemokine Expression and Proliferation in Human Vascular Smooth Muscle Cells."

2010: CARIM PhD award

Competitive personal grant for 4 years doctorate training 